SCIPP $17 / 11$

\title{
Supersymmetric Theory and Models
}

\author{
Howard E. Haber ${ }^{1}$ and Laurel Stephenson Haskins ${ }^{1,2}$ \\ ${ }^{1}$ Santa Cruz Institute for Particle Physics, \\ University of California, Santa Cruz, CA 95064, USA \\ ${ }^{2}$ Racah Institute of Physics, \\ Hebrew University, Jerusalem 91904, Israel
}

\begin{abstract}
In these introductory lectures, we review the theoretical tools used in constructing supersymmetric field theories and their application to physical models. We first introduce the technology of two-component spinors, which is convenient for describing spin- $\frac{1}{2}$ fermions. After motivating why a theory of nature may be supersymmetric at the TeV energy scale, we show how supersymmetry (SUSY) arises as an extension of the Poincaré algebra of spacetime symmetries. We then obtain the representations of the SUSY algebra and discuss its simplest realization in the WessZumino model. In order to have a systematic approach for obtaining supersymmetric Lagrangians, we introduce the formalism of superspace and superfields and recover the Wess-Zumino Lagrangian. These methods are then extended to encompass supersymmetric abelian and nonabelian gauge theories coupled to supermatter. Since supersymmetry is not an exact symmetry of nature, it must ultimately be broken. We discuss several mechanisms of SUSY-breaking (both spontaneous and explicit) and briefly survey various proposals for realizing SUSY-breaking in nature. Finally, we construct the the Minimal Supersymmetric extension of the Standard Model (MSSM), and consider the implications for the future of SUSY in particle physics.
\end{abstract}

\section{Contents}

Supersymmetric Theory and Models

1. Introduction to the TASI-2016 Supersymmetry Lectures . . . . . . . . . . . . 3

2. Spin-1/2 fermions in quantum field theory . . . . . . . . . . . . . . . . . . . 4

2.1. Two-component spinor technology . . . . . . . . . . . . . . . . 6

2.2. Correspondence between the two- and four-component spinor notations . 16

2.3. Feynman Rules for Dirac and Majorana fermions . . . . . . . . . . . . . 20

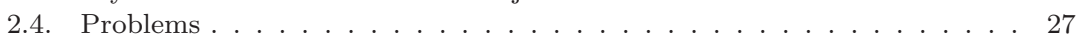


3. Motivation for TeV-scale supersymmetry . . . . . . . . . . . . . . . . 28

3.1. Why the TeV scale? . . . . . . . . . . . . . . . . . . . 28

3.2. The modern principle of naturalness . . . . . . . . . . . . . . . 30

3.3. Avoiding quadratic UV-sensitivity with elementary scalars . . . . . . . . 31

4. Supersymmetry: first steps . . . . . . . . . . . . . . . . . . . . . . 32

4.1. Review of the Poincaré algebra . . . . . . . . . . . . . . . . . . . . . . . . . . 32

4.2. The supersymmetry (SUSY) algebra . . . . . . . . . . . . . . . 34

4.3. Representations of the $N=1$ SUSY algebra . . . . . . . . . . . . . . . 37

4.4. Consequences of super-Poincaré invariance . . . . . . . . . . . . . . . . 44

4.5. Supersymmetric theories of spin-0 and spin- $\frac{1}{2}$ particles . . . . . . . . . 49

4.6. The SUSY algebra realized off-shell . . . . . . . . . . . . . . . . . . . 52

4.7. Counting bosonic and fermionic degrees of freedom . . . . . . . . . . . . 54

4.8. Lessons from the Wess-Zumino Model . . . . . . . . . . . . . . . . . 55

4.9. Appendix: Constructing the states of a supermultiplet . . . . . . . . 55

4.10. Problems . . . . . . . . . . . . . . . . . 6 6 . . . . . . . . . . . . . 6

5. Superspace and Superfields . . . . . . . . . . . . . . . . . . . . . . . 61

5.1. Superspace coordinates and translations . . . . . . . . . . . . . . 61

5.2. Expansion of the superfield in powers of $\theta$ and $\theta^{\dagger} \ldots . . . . . . . . .664$

5.3. Spinor covariant derivatives . . . . . . . . . . . . . . . 65

5.4. Chiral superfields ........................ 67

5.5. Constructing the SUSY Lagrangian . . . . . . . . . . . . . . . . . 70

5.6. R-invariance ....................... . . 74

5.7. Grassmann integration and the SUSY action . . . . . . . . . . . . . . 75

5.8. Improved ultraviolet behavior of supersymmetry . . . . . . . . . . . . . 77

5.9. Problems . . . . . . . . . . . . . . . . . . . 79

6. Supersymmetric gauge theories . . . . . . . . . . . . . . . . . . . . . . . . . . . . . . . . . .

6.1. Vector superfields . . . . . . . . . . . . . . . . . . . . 81

6.2. Gauge invariance . . . . . . . . . . . . . . . . . . . . . . 84

6.3. Gauge-invariant interactions . . . . . . . . . . . . . . . . . . 86

6.4. Generalizing to more than one chiral superfield . . . . . . . . . . . . . 87

6.5. SUSY Yang-Mills theory coupled to supermatter . . . . . . . . . . . . . 89

6.6. The SUSY Lagrangian . . . . . . . . . . . . . . . . . . . . . . . . . . . . . . . . . . . . . . . . .

6.7. Problems . . . . . . . . . . . . . . . . . . . 92

7. Supersymmetry Breaking . . . . . . . . . . . . . . . . . . . 93

7.1. Spontaneous SUSY breaking . . . . . . . . . . . . . . . . 93

7.2. Mass Sum rules . . . . . . . . . . . . . . . . . . . . 97

7.3. The origin of SUSY-breaking dynamics . . . . . . . . . . . . . . . 99

7.4. A phenomenological approach: soft SUSY-breaking . . . . . . . . . . 102

7.5. Problems . . . . . . . . . . . . . . . 106

8. Supersymmetric extension of the Standard Model (MSSM) . . . . . . . . . . 107

8.1. Field content of the MSSM . . . . . . . . . . . . . . . . 108

8.2. The superpotential of the MSSM . . . . . . . . . . . . . . 111

8.3. Supersymmetry breaking in the MSSM . . . . . . . . . . . . . . . 114

8.4. The MSSM parameter count . . . . . . . . . . . . . . . . 115

8.5. The MSSM particle spectrum . . . . . . . . . . . . . . . . . . . . . . . . . . . . . . . . . . . . . . . . . . . .

8.6. The Higgs sector of the MSSM . . . . . . . . . . . . . . . . . . . . 120

8.7. Unification of gauge couplings . . . . . . . . . . . . . . . . . . . . 124

8.8. Problems . . . . . . . . . . . . . . . . . . . . . 128

9. Supersymmetry Quo Vadis? . . . . . . . . . . . . . . . . . . . . . . 129

References........................... . . 135 


\section{Introduction to the TASI-2016 Supersymmetry Lectures}

These lectures were first presented at the 2016 Theoretical Advanced Study Institute (TASI-2016) in Boulder, CO. Four ninety-minute lectures were given, with the aim of presenting the basic theoretical techniques of supersymmetry needed for the construction of a supersymmetric extension of the Standard Model of particle physics. The lectures were pitched at an elementary level, assuming that the students were well versed in quantum field theory, gauge theory and the Standard Model, but with no assumed prior knowledge of supersymmetry. Nevertheless, some aspects of these lectures may also be useful to the reader with some prior knowledge of supersymmetry.

It is possible to introduce the technology of supersymmetry theory using four-component spinor notation that is familiar to all students of quantum field theory. However, it is our view that employing two-component spinor notation greatly simplifies the presentation of the theoretical structure of supersymmetry in $3+1$ spacetime dimensions. Thus, in Section 2, we introduce the two-component spinor notation in some detail and discuss how it is related to the better known four-component spinor notation. This material is based heavily on a comprehensive review of Dreiner, Haber and Martin that is presented in Ref. [1]. In this review, it is shown that practical calculations in quantum field theory can be carried out entirely within the framework of the two-component spinor notation, which include the development of Feynman rules for two-component spinors. However, at the end of Section 1, we are slightly less ambitious and revert to fourcomponent fermion notation for the purpose of computing scattering and decay processes. In particular, we provide a translation between two and four-component spinor notation, and develop four-component spinor Feynman rules that treat both Dirac and Majorana fermions on the same footing.

In Section 3, we present the motivation for TeV-scale supersymmetry. Namely, why is it that we feel compelled to introduce a supersymmetric extension of the Standard Model, despite the great success of the Standard Model in describing collider data and the absence of significant evidence for new physics beyond the Standard Model. With this motivation in mind, we are ready to explore the theoretical aspects of supersymmetry.

Since this is not a review article, we do not feel compelled to present a comprehensive list of references. Nevertheless, it is instructive to assemble a list of books and lecture notes on supersymmetry, many of which we have found quite useful in preparing these lectures. Thus, we draw your attention 
to the following books listed in Refs. [2-29] and the following reviews and lecture notes listed in Ref. [30-49]. The reader is warned that conventions vary widely among these references. Apart from the two possible choices for the spacetime metric (either the mostly minus metric used in these lectures or the mostly plus metric), there are many different choices in the definition of a variety of quantities, often involving different choices of signs. Of these many conventions, we believe that the ones employed in these lecture notes are probably closest to those that appear in Ref. [33]. ${ }^{1}$

In Section 4, we show how the algebra of the Poincaré group can be extended to obtain the supersymmetry (SUSY) algebra. The representations of the $N=1$ SUSY algebra are elucidated, and the Wess-Zumino model is presented as the simplest realization of a supersymmetric field theory. In Section 5, we take some of the mystery out of constructing a SUSY Lagrangian by introducing the concepts of superspace and superfields. This formalism allows one to construct supersymmetric field theories without any guesswork. In Section 6, the formalism of supersymmetric gauge theories is developed. In Section 7, we examine supersymmetry breaking, which is necessary for accommodating the observation that the elementary particles observed today are not each accompanied by an equal-mass superpartner. Finally, in Section 8, we construct the Minimal Supersymmetric extension of the Standard Model (MSSM). We end these lectures in Section 9 with a brief discussion of what lies ahead for supersymmetry.

\section{Spin-1/2 fermions in quantum field theory}

We begin these lectures with a treatment of spin- $\frac{1}{2}$ fermions in quantum field theory. In most introductory courses in relativistic quantum field theory, the student first encounters fermion fields in the treatment of a relativistic theory of electrons and photons. The electron is represented by a four-component Dirac fermion field, and the free field electron Lagrangian yields the Dirac equation. The four components represent two degrees of freedom corresponding to the electron and two degrees of freedom corresponding to the positron. Feynman rules for quantum electrodynamics are developed and the vector-like nature of the $e^{+} e^{-}$coupling to photons leads to some important simplifications.

\footnotetext{
${ }^{1}$ We also note that although Ref. [38] employs the mostly plus metric, one can obtain a version of Martin's Supersymmetry Primer in the mostly minus metric by changing one line in the LaTeX source code. This alternative version of the Primer closely matches the conventions employed in these lectures.
} 
The theory of electroweak interactions involves chiral interactions of fermions with gauge bosons. Left-handed and right-handed fermions transform differently under the electroweak gauge group, which may appear strange to students trained to think in terms of four-component Dirac fermions. Nevertheless, after electroweak symmetry breaking, the masseigenstate fermion fields can be identified. All massive fermion states are charged under $\mathrm{U}(1)_{\mathrm{EM}}$ and are thus represented by Dirac fermion fields. The neutrinos are massless, but only the left-handed neutrinos and righthanded antineutrinos are present in the theory. Thus, one can still use fourcomponent fermion fields (by applying the appropriate chiral projection operators on the neutrino fields). Hence, the four-component techniques of quantum electrodynamics are easily accommodated and Feynman rules for the fermion fields are obtained in a straightforward manner.

However, the observation of neutrino mixing phenomena implies that neutrinos are massive, which requires new physics beyond the Standard Model of the electroweak interactions. Models of neutrino mass often include neutral self-conjugate fermion states with two degrees of freedom, called Majorana fermions. Such states can be described using fourcomponent fermion fields that are constrained by an appropriate conjugation condition. However, the resulting field theory description of systems of Majorana and Dirac fermions is somewhat awkward. Moreover, the Feynman rules for interacting Majorana fermions require some care.

Returning to first principles, one can ask how spin- $\frac{1}{2}$ fermions arise in quantum field theory. In Section 2.1, we shall demonstrate that the fundamental building blocks employed in constructing spin- $\frac{1}{2}$ quantum fields are two-component spinors corresponding to the two-dimensional representations of the Lorentz group. A neutral Majorana fermion is then represented by a two-component fermion field. Dirac fermions arise when one considers theories of two mass-degenerate two-component fermions, which can be combined to make a charged four-component Dirac fermion. This is completely analogous to the case of spin-0 bosons, in which a neutral boson is represented by a real scalar field and a charged boson is represented by a complex scalar field (whose real and imaginary parts constitute two mass-degenerate real scalars).

The development of two-component spinor technology has a number of benefits. First, it provides an elegant unified description of Majorana and Dirac fermions. Second, it is very convenient to employ the two-component spinor formalism in theories of chiral interactions. Finally, it will prove especially useful in developing the formalism of supersymmetry, which is 
the main focus of these lectures.

Because most students see the four-component spinor formalism first and are therefore more familiar with it, we shall devote Section 2.2 to the translation between the two- and four-component formalisms. Finally, in Section 2.3 we demonstrate how Feynman rules involving four-component fermion fields can be extended to incorporate Majorana fermions.

This section is based on a comprehensive review of Dreiner, Haber and Martin [1], where many references to the original literature can be found.

\subsection{Two-component spinor technology}

\subsubsection{Orthochronous Lorentz transformations}

Quantum spin- $\frac{1}{2}$ fields transform under a two-dimensional irreducible representation of the Lorentz group. Thus, we first examine the properties that define a Lorentz transformation [50]. Under an active Lorentz transformation, $\Lambda_{\nu}^{\mu}$, a four-vector $p^{\mu}$ transforms as

$$
p^{\prime \mu}=\Lambda_{\nu}^{\mu} p^{\nu} .
$$

The condition that $g_{\mu \nu} p^{\mu} p^{\nu}$ is invariant under Lorentz transformations implies that ${ }^{2}$

$$
\Lambda_{\nu}^{\mu} g_{\mu \rho} \Lambda_{\lambda}^{\rho}=g_{\nu \lambda} .
$$

That is, $\Lambda \in \mathrm{O}(3,1)$. Eq. (2.2) implies that $\Lambda$ possesses the following two properties: (i) $\operatorname{det} \Lambda= \pm 1$ and (ii) $\left|\Lambda^{0}{ }_{0}\right| \geq 1$. Thus, Lorentz transformations fall into four disconnected classes denoted by a pair of signs, $\left(\operatorname{sgn}[\operatorname{det} \Lambda], \operatorname{sgn}\left[\Lambda^{0}{ }_{0}\right]\right)$. The proper orthochronous Lorentz transformations correspond to $(+,+)$ and are continuously connected to the identity.

The most general proper orthochronous Lorentz transformation, characterized by a rotation angle $\theta$ about an axis $\widehat{\boldsymbol{n}}(\overrightarrow{\boldsymbol{\theta}} \equiv \theta \widehat{\boldsymbol{n}})$ and a boost vector $\overrightarrow{\boldsymbol{\zeta}} \equiv \hat{\boldsymbol{v}} \tanh ^{-1} \beta$ (where $\hat{\boldsymbol{v}} \equiv \overrightarrow{\boldsymbol{v}} /|\overrightarrow{\boldsymbol{v}}|$ is the unit velocity vector and $\beta \equiv|\overrightarrow{\boldsymbol{v}}| / c),{ }^{3}$ is a $4 \times 4$ matrix given by:

$$
\Lambda=\exp \left(-\frac{1}{2} i \theta^{\alpha \beta} s_{\alpha \beta}\right)=\exp (-i \overrightarrow{\boldsymbol{\theta}} \cdot \overrightarrow{\boldsymbol{s}}-i \overrightarrow{\boldsymbol{\zeta}} \cdot \overrightarrow{\boldsymbol{k}}),
$$

where $\theta^{\alpha \beta}$ is antisymmetric, with $\theta^{i} \equiv \frac{1}{2} \epsilon^{i j k} \theta_{j k}, \zeta^{i} \equiv \theta^{i 0}=-\theta^{0 i}$, and

$$
\left(s_{\alpha \beta}\right)^{\mu}{ }_{\nu}=i\left(g_{\alpha}{ }^{\mu} g_{\beta \nu}-g_{\beta}{ }^{\mu} g_{\alpha \nu}\right) \text {, }
$$

with $s^{i} \equiv \frac{1}{2} \epsilon^{i j k} s_{j k}$ and $k^{i} \equiv s^{0 i}=-s^{i 0}$. We have employed a notation where the lower case Latin indices $i, j, k=1,2,3$ and $\epsilon^{123}=+1$.

${ }^{2}$ In our conventions, the Minkowski metric tensor is $g_{\mu \nu}=\operatorname{diag}(1,-1,-1,-1)$.

${ }^{3}$ Henceforth, we shall work in particle physics units where $\hbar=c=1$. 
Note that the $s^{\mu \nu}$ are antisymmetric $4 \times 4$ matrices, i.e., $s^{\mu \nu}=-s^{\nu \mu}$, and satisfy the commutation relations,

$$
\left[s^{\alpha \beta}, s^{\rho \sigma}\right]=i\left(g^{\beta \rho} s^{\alpha \sigma}-g^{\alpha \rho} s^{\beta \sigma}-g^{\beta \sigma} s^{\alpha \rho}+g^{\alpha \sigma} s^{\beta \rho}\right) .
$$

It follows from eqs. (2.3) and (2.4) that an infinitesimal orthochronous Lorentz transformation is given by

$$
\Lambda^{\mu}{ }_{\nu} \simeq \delta^{\mu}{ }_{\nu}+\theta^{\mu}{ }_{\nu} \simeq\left(\mathbb{1}_{4 \times 4}-i \overrightarrow{\boldsymbol{\theta}} \cdot \overrightarrow{\boldsymbol{s}}-i \overrightarrow{\boldsymbol{\zeta}} \cdot \overrightarrow{\boldsymbol{k}}\right)^{\mu}{ }_{\nu},
$$

where $\mathbb{1}_{4 \times 4}$ is the $4 \times 4$ identity matrix, and we have used $\theta^{\mu}{ }_{\nu}=-\theta_{\nu}{ }^{\mu}$.

\subsubsection{Finite-dimensional Representations of the Lorentz Group}

A generic spin- $s$ field $\Phi$ transforms as

$$
\Phi(x) \rightarrow \Phi^{\prime}\left(x^{\prime}\right)=M_{R}(\Lambda) \Phi(x),
$$

where $M_{R} \equiv \exp \left(-\frac{1}{2} i \theta_{\mu \nu} S^{\mu \nu}\right)$ and the $S_{\mu \nu}$ constitute finite-dimensional irreducible matrix representations of the Lie algebra of the Lorentz group. The $S^{\mu \nu}$ satisfy the same commutation relations as the $s^{\mu \nu}$ given in eq. (2.5). It is convenient to denote the six independent generators defined by the $S^{\mu \nu}$ as

$$
S^{i} \equiv \frac{1}{2} \epsilon^{i j k} S_{j k}, \quad K^{i} \equiv S^{0 i},
$$

where $i, j, k=1,2,3$. The $S^{i}$ generate three-dimensional rotations in space and the $K^{i}$ generate the Lorentz boosts. It then follows that

$$
M_{R} \equiv \exp (-i \overrightarrow{\boldsymbol{\theta}} \cdot \overrightarrow{\boldsymbol{S}}-i \overrightarrow{\boldsymbol{\zeta}} \cdot \overrightarrow{\boldsymbol{K}}) .
$$

The $S^{i}$ and $K^{i}$ satisfy the commutation relations,

$$
\begin{aligned}
{\left[S^{i}, S^{j}\right] } & =\epsilon^{i j k} S^{k}, \\
{\left[S^{i}, K^{j}\right] } & =\epsilon^{i j k} K^{k}, \\
{\left[K^{i}, K^{j}\right] } & =-\epsilon^{i j k} S^{k} .
\end{aligned}
$$

We define the following linear combinations of the generators,

$$
\overrightarrow{\boldsymbol{S}}_{+} \equiv \frac{1}{2}(\overrightarrow{\boldsymbol{S}}+i \overrightarrow{\boldsymbol{K}}), \quad \overrightarrow{\boldsymbol{S}}_{-} \equiv \frac{1}{2}(\overrightarrow{\boldsymbol{S}}-i \overrightarrow{\boldsymbol{K}}),
$$

which satisfy the commutation relations,

$$
\begin{aligned}
& {\left[S_{+}^{i}, S_{+}^{j}\right]=i \epsilon^{i j k} S_{+}^{k},} \\
& {\left[S_{-}^{i}, S_{-}^{j}\right]=i \epsilon^{i j k} S_{-}^{k},} \\
& {\left[S_{ \pm}^{i}, S_{\mp}^{j}\right]=0,}
\end{aligned}
$$

corresponding to two independent (complexified) SU(2) Lie algebras. Thus, the representations of the Lorentz algebra are characterized by $\left(s_{1}, s_{2}\right)$, where the $s_{i}$ are half-integers. For example, $(0,0)$ corresponds to a scalar field and $\left(\frac{1}{2}, \frac{1}{2}\right)$ corresponds to a four-vector field. 


\subsubsection{Two-component spinors}

Spin-1/2 fermion fields transform under the spinor representations, $\left(\frac{1}{2}, 0\right)$ corresponding to $\overrightarrow{\boldsymbol{S}}_{+}=\frac{1}{2} \overrightarrow{\boldsymbol{\sigma}}$ and $\overrightarrow{\boldsymbol{S}}_{-}=0$, and $\left(0, \frac{1}{2}\right)$ corresponding to $\overrightarrow{\boldsymbol{S}}_{+}=0$ and $\overrightarrow{\boldsymbol{S}}_{-}=\frac{1}{2} \overrightarrow{\boldsymbol{\sigma}}$. That is, the Lorentz transformation matrices acting on spinor fields may be written in terms of the Pauli spin matrices $\sigma^{1}, \sigma^{2}$, and $\sigma^{3}$ as follows,

$$
\left(\frac{1}{2}, 0\right): \quad M=\exp \left(-\frac{i}{2} \overrightarrow{\boldsymbol{\theta}} \cdot \overrightarrow{\boldsymbol{\sigma}}-\frac{1}{2} \overrightarrow{\boldsymbol{\zeta}} \cdot \overrightarrow{\boldsymbol{\sigma}}\right),
$$

which via a similarity transformation is equivalent to the matrix representation, $\left(M^{-1}\right)^{\top}=i \sigma^{2} M\left(i \sigma^{2}\right)^{-1}$, and

$$
\left(0, \frac{1}{2}\right): \quad\left[M^{-1}\right]^{\dagger}=\exp \left(-\frac{i}{2} \overrightarrow{\boldsymbol{\theta}} \cdot \overrightarrow{\boldsymbol{\sigma}}+\frac{1}{2} \overrightarrow{\boldsymbol{\zeta}} \cdot \overrightarrow{\boldsymbol{\sigma}}\right),
$$

which via a similarity transformation is equivalent to the matrix representation, $M^{*}=i \sigma^{2}\left[M^{-1}\right]^{\dagger}\left(i \sigma^{2}\right)^{-1}$.

Thus, the Lorentz transformation law for two-component $\left(\frac{1}{2}, 0\right)$ fields can be written in two equivalent ways,

$$
\xi_{\alpha}^{\prime}=M_{\alpha}{ }^{\beta} \xi_{\beta}, \quad \xi^{\prime \alpha}=\left[\left(M^{-1}\right)^{\top}\right]^{\alpha}{ }_{\beta} \xi^{\beta},
$$

where $\alpha, \beta=1,2$. Likewise, the Lorentz transformation law for twocomponent $\left(0, \frac{1}{2}\right)$ fields can be written in two equivalent ways,

$$
\xi^{\prime \dagger \dot{\alpha}}=\left[\left(M^{-1}\right)^{\dagger}\right]_{\dot{\beta}}^{\dot{\alpha}} \xi^{\dagger \dot{\beta}}, \quad \xi_{\dot{\alpha}}^{\prime \dagger}=\left[M^{*}\right]_{\dot{\alpha}} \dot{\beta}_{\dot{\beta}}^{\dagger} .
$$

The $\left(0, \frac{1}{2}\right)$ fields are related to the $\left(\frac{1}{2}, 0\right)$ fields by hermitian conjugation,

$$
\xi_{\dot{\alpha}}^{\dagger} \equiv\left(\xi_{\alpha}\right)^{\dagger}, \quad \xi^{\dagger \dot{\alpha}} \equiv\left(\xi^{\alpha}\right)^{\dagger} .
$$

It is conventional to employ undotted indices for the spinor components of $\left(\frac{1}{2}, 0\right)$ fields and dotted indices for the spinor components of $\left(0, \frac{1}{2}\right)$ fields.

As noted below eqs. (2.17) and (2.18), respectively, each of the two equivalent representation matrices, $M$ and $\left(M^{-1}\right)^{\top}$ in the case of $\left(\frac{1}{2}, 0\right)$, and $\left(M^{-1}\right)^{\dagger}$ and $M^{*}$ in the case of $\left(0, \frac{1}{2}\right)$, are related by a similarity transformation involving the antisymmetric matrices,

$$
i \sigma^{2}=\left(\begin{array}{rr}
0 & 1 \\
-1 & 0
\end{array}\right)=\epsilon^{\alpha \beta}=\epsilon^{\dot{\alpha} \dot{\beta}},
$$

and

$$
\left(i \sigma^{2}\right)^{-1}=-i \sigma^{2}=\epsilon_{\alpha \beta}=\epsilon_{\dot{\alpha} \dot{\beta}},
$$

which define the epsilon symbols with undotted and dotted indices. Note that the epsilon symbols with raised and lowered indices differ by an overall sign. Moreover, they can be used to raise and lower the spinor indices, 


$$
\xi^{\alpha}=\epsilon^{\alpha \beta} \xi_{\beta}, \quad \xi_{\alpha}=\epsilon_{\alpha \beta} \xi^{\beta}, \quad \xi^{\dagger \dot{\alpha}}=\epsilon^{\dot{\alpha} \dot{\beta}} \xi_{\dot{\beta}}^{\dagger}, \quad \xi_{\dot{\alpha}}^{\dagger}=\epsilon_{\dot{\alpha} \dot{\beta}} \xi^{\dagger \dot{\beta}} .
$$

The products of two epsilon symbols with undotted and with dotted indices, respectively, satisfy,

$$
\begin{aligned}
& \epsilon_{\alpha \beta} \epsilon^{\gamma \delta}=-\delta_{\alpha}^{\gamma} \delta_{\beta}^{\delta}+\delta_{\alpha}^{\delta} \delta_{\beta}^{\gamma}, \\
& \epsilon_{\dot{\alpha} \dot{\beta}} \epsilon^{\dot{\gamma} \dot{\delta}}=-\delta_{\dot{\alpha}}^{\dot{\gamma}} \delta_{\dot{\beta}}^{\dot{\delta}}+\delta_{\dot{\alpha}}^{\dot{\delta}} \delta_{\dot{\beta}}^{\dot{\gamma}},
\end{aligned}
$$

where $\delta_{\dot{\alpha}}^{\dot{\beta}}=\delta_{\alpha}^{\beta}$ and the two-index symmetric Kronecker delta symbol with undotted indices is defined by $\delta_{1}^{1}=\delta_{2}^{2}=1$ and $\delta_{1}^{2}=\delta_{2}^{1}=0$. In particular,

$$
\epsilon_{\alpha \gamma} \epsilon^{\gamma \beta}=\delta_{\alpha}^{\beta}, \quad \epsilon_{\dot{\alpha} \dot{\gamma}} \epsilon^{\dot{\gamma} \dot{\beta}}=\delta_{\dot{\alpha}}^{\dot{\beta}} .
$$

Finally, we introduce the $\sigma$-matrices:

$$
\sigma_{\alpha \dot{\beta}}^{\mu}=\left(\mathbb{1}_{2 \times 2} ; \overrightarrow{\boldsymbol{\sigma}}\right), \quad \bar{\sigma}^{\mu \dot{\alpha} \beta}=\left(\mathbb{1}_{2 \times 2} ;-\overrightarrow{\boldsymbol{\sigma}}\right),
$$

where $\mathbb{1}_{2 \times 2}$ is the $2 \times 2$ identity matrix. The spinor index structure derives from the relations,

$$
\left(M^{\dagger}\right)^{\dot{\alpha}}{ }_{\dot{\beta}} \bar{\sigma}^{\mu \dot{\beta} \gamma} M_{\gamma}{ }^{\delta}=\Lambda_{\nu}^{\mu}{ }_{\nu} \bar{\sigma}^{\nu \dot{\alpha} \delta}, \quad\left(M^{-1}\right)_{\alpha}{ }^{\beta} \sigma_{\beta \dot{\gamma}}^{\mu}\left[\left(M^{-1}\right)^{\dagger}\right]^{\dot{\gamma}} \dot{\delta}=\Lambda^{\mu}{ }_{\nu} \sigma_{\alpha \dot{\delta}}^{\nu} .
$$

Note that the matrix $M$ and its inverse have the same spinor index structure (and likewise for the matrix $M^{\dagger}$ and its inverse).

We will sometimes find it useful to relate the $\sigma^{\mu}$ and $\bar{\sigma}^{\mu}$ matrices using the identities

$$
\sigma_{\alpha \dot{\alpha}}^{\mu}=\epsilon_{\alpha \beta} \epsilon_{\dot{\alpha} \dot{\beta}} \bar{\sigma}^{\mu \dot{\beta} \beta}, \quad \bar{\sigma}^{\mu \dot{\alpha} \alpha}=\epsilon^{\alpha \beta} \epsilon^{\dot{\alpha} \dot{\beta}} \sigma_{\beta \dot{\beta}}^{\mu} .
$$

The significance of $\sigma^{\mu}$ is that Lorentz 4 -vectors can be built from spinor bilinears. For example, $\chi^{\alpha}(x) \sigma_{\alpha \dot{\beta}}^{\mu} \dot{\xi}^{\dot{\beta}}(x)$ transforms as a Lorentz 4-vector,

$$
\begin{aligned}
\chi^{\prime \alpha}\left(x^{\prime}\right) \sigma_{\alpha \dot{\beta}}^{\mu} \xi^{\prime \dagger \dot{\beta}}\left(x^{\prime}\right) & =\chi^{\alpha}(x)\left[M^{-1} \sigma^{\mu}\left(M^{-1}\right)^{\dagger}\right]_{\alpha \dot{\beta}} \xi^{\dagger \dot{\beta}}(x) \\
& =\Lambda^{\mu}{ }_{\nu} \chi(x)^{\alpha} \sigma_{\alpha \dot{\beta}}^{\nu} \xi^{\dagger \dot{\beta}}(x),
\end{aligned}
$$

after making use of eq. (2.29). Spinor indices can be suppressed by adopting a summation convention where we contract indices as follows:

$$
\alpha_{\alpha} \text { and } \dot{\alpha}^{\dot{\alpha}} \text {. }
$$

For example,

$$
\begin{aligned}
\xi \eta & \equiv \xi^{\alpha} \eta_{\alpha}, \\
\xi^{\dagger} \eta^{\dagger} & \equiv \xi_{\dot{\alpha}}^{\dagger} \eta^{\dagger \dot{\alpha}}, \\
\xi^{\dagger} \bar{\sigma}^{\mu} \eta & \equiv \xi_{\dot{\alpha}}^{\dagger} \bar{\sigma}^{\mu \dot{\alpha} \beta} \eta_{\beta}, \\
\xi \sigma^{\mu} \eta^{\dagger} & \equiv \xi^{\alpha} \sigma_{\alpha \dot{\beta}}^{\mu} \eta^{\dagger \dot{\beta}} .
\end{aligned}
$$


In particular, for anticommuting spinors,

$$
\begin{aligned}
\eta \xi \equiv \eta^{\alpha} \xi_{\alpha} & =-\xi_{\alpha} \eta^{\alpha}=+\xi^{\alpha} \eta_{\alpha}=\xi \eta . \\
\eta^{\dagger} \xi^{\dagger} \equiv \eta_{\dot{\alpha}}^{\dagger} \xi^{\dagger^{\dot{\alpha}}} & =-\xi^{\dagger} \eta_{\dot{\alpha}}^{\dagger}=\xi_{\dot{\alpha}}^{\dagger} \eta^{\dagger \dot{\alpha}}=\xi^{\dagger} \eta^{\dagger} .
\end{aligned}
$$

The behavior of spinor products under hermitian conjugation is noteworthy,

$$
(\xi \Sigma \eta)^{\dagger}=\eta^{\dagger} \Sigma_{r} \xi^{\dagger}, \quad\left(\xi \Sigma \eta^{\dagger}\right)^{\dagger}=\eta \Sigma_{r} \xi^{\dagger}, \quad\left(\xi^{\dagger} \Sigma \eta\right)^{\dagger}=\eta^{\dagger} \Sigma_{r} \xi,
$$

where in each case $\Sigma$ stands for any sequence of alternating $\sigma$ and $\bar{\sigma}$ matrices, and $\Sigma_{r}$ is obtained by reversing the order of the $\sigma$ and $\bar{\sigma}$ matrices that appear in $\Sigma$.

From the sigma matrices, one can construct the antisymmetrized products,

$$
\begin{aligned}
& \left(\sigma^{\mu \nu}\right)_{\alpha}^{\beta} \equiv \frac{1}{4} i\left(\sigma_{\alpha \dot{\gamma}}^{\mu} \bar{\sigma}^{\nu \dot{\gamma} \beta}-\sigma_{\alpha \dot{\gamma}}^{\nu} \bar{\sigma}^{\mu \dot{\gamma} \beta}\right), \\
& \left(\bar{\sigma}^{\mu \nu}\right)_{\dot{\beta}}^{\dot{\alpha}} \equiv \frac{1}{4} i\left(\bar{\sigma}^{\mu \dot{\alpha} \gamma} \sigma_{\gamma \dot{\beta}}^{\nu}-\bar{\sigma}^{\nu \dot{\alpha} \gamma} \sigma_{\gamma \dot{\beta}}^{\mu}\right) .
\end{aligned}
$$

With this notation, we may write the $\left(\frac{1}{2}, 0\right)$ and $\left(0, \frac{1}{2}\right)$ transformation matrices, respectively, as

$$
\begin{aligned}
M & =\exp \left(-\frac{1}{2} i \theta^{\mu \nu} \sigma_{\mu \nu}\right), \\
\left(M^{-1}\right)^{\dagger} & =\exp \left(-\frac{1}{2} i \theta^{\mu \nu} \bar{\sigma}_{\mu \nu}\right),
\end{aligned}
$$

where the $\theta^{\mu \nu}$ are defined below eq. (2.3).

Consider a pure boost of an on-shell two-component spinor from its rest frame to the frame where $p^{\mu}=\left(E_{\boldsymbol{p}}, \overrightarrow{\boldsymbol{p}}\right)$, with $E_{\boldsymbol{p}}=\left(|\overrightarrow{\boldsymbol{p}}|^{2}+m^{2}\right)^{1 / 2}$. In this case, setting $\theta^{i j}=0$ (corresponding to no rotation), we obtain,

$$
\begin{aligned}
M & =\exp \left(-\frac{1}{2} \overrightarrow{\boldsymbol{\zeta}} \cdot \overrightarrow{\boldsymbol{\sigma}}\right)=\sqrt{\frac{p \cdot \sigma}{m}}=\frac{\left(E_{\boldsymbol{p}}+m\right) \mathbb{1}_{2 \times 2}-\overrightarrow{\boldsymbol{\sigma}} \cdot \vec{p}}{\sqrt{2 m\left(E_{\boldsymbol{p}}+m\right)}}, \\
\left(M^{-1}\right)^{\dagger} & =\exp \left(+\frac{1}{2} \overrightarrow{\boldsymbol{\zeta}} \cdot \overrightarrow{\boldsymbol{\sigma}}\right)=\sqrt{\frac{p \cdot \bar{\sigma}}{m}}=\frac{\left(E_{\boldsymbol{p}}+m\right) \mathbb{1}_{2 \times 2}+\overrightarrow{\boldsymbol{\sigma}} \cdot \vec{p}}{\sqrt{2 m\left(E_{\boldsymbol{p}}+m\right)}} .
\end{aligned}
$$

The matrix square roots, $\sqrt{p \cdot \sigma}$ and $\sqrt{p \cdot \bar{\sigma}}$, appearing in eqs. (2.45) and (2.46) are defined to be the unique non-negative definite hermitian matrices whose squares are equal to the non-negative definite hermitian matrices $p \cdot \sigma$ and $p \cdot \bar{\sigma}$, respectively. ${ }^{4}$

\footnotetext{
${ }^{4}$ Note that $p \cdot \sigma$ and $p \cdot \bar{\sigma}$ are non-negative matrices due to the implicit mass-shell condition satisfied by $p^{\mu}$.
} 


\subsubsection{Useful identities}

The following identities can be used to systematically simplify expressions involving products of $\sigma$ and $\bar{\sigma}$ matrices,

$$
\begin{aligned}
& \sigma_{\alpha \dot{\alpha}}^{\mu} \bar{\sigma}_{\mu}^{\dot{\beta} \beta}=2 \delta_{\alpha}^{\beta} \delta_{\dot{\alpha}}^{\dot{\beta}}, \\
& \sigma_{\alpha \dot{\alpha}}^{\mu} \sigma_{\mu \beta \dot{\beta}}=2 \epsilon_{\alpha \beta} \epsilon_{\dot{\alpha} \dot{\beta}}, \\
& \bar{\sigma}^{\mu \dot{\alpha} \alpha} \bar{\sigma}_{\mu}^{\dot{\beta} \beta}=2 \epsilon^{\alpha \beta} \epsilon^{\dot{\alpha} \dot{\beta}}, \\
& {\left[\sigma^{\mu} \bar{\sigma}^{\nu}+\sigma^{\nu} \bar{\sigma}^{\mu}\right]_{\alpha}^{\beta}=2 g^{\mu \nu} \delta_{\alpha}^{\beta},} \\
& {\left[\bar{\sigma}^{\mu} \sigma^{\nu}+\bar{\sigma}^{\nu} \sigma^{\mu}\right]_{\dot{\beta}}^{\dot{\alpha}}=2 g^{\mu \nu} \delta_{\dot{\beta}}^{\dot{\alpha}},} \\
& \sigma^{\mu} \bar{\sigma}^{\nu} \sigma^{\rho}=g^{\mu \nu} \sigma^{\rho}-g^{\mu \rho} \sigma^{\nu}+g^{\nu \rho} \sigma^{\mu}+i \epsilon^{\mu \nu \rho \kappa} \sigma_{\kappa}, \\
& \bar{\sigma}^{\mu} \sigma^{\nu} \bar{\sigma}^{\rho}=g^{\mu \nu} \bar{\sigma}^{\rho}-g^{\mu \rho} \bar{\sigma}^{\nu}+g^{\nu \rho} \bar{\sigma}^{\mu}-i \epsilon^{\mu \nu \rho \kappa} \bar{\sigma}_{\kappa},
\end{aligned}
$$

where $\epsilon^{0123}=-\epsilon_{0123}=+1$ in our conventions. The traces of alternating products of $\sigma$ and $\bar{\sigma}$ matrices are given by,

$$
\begin{aligned}
& \operatorname{Tr}\left[\sigma^{\mu} \bar{\sigma}^{\nu}\right]=\operatorname{Tr}\left[\bar{\sigma}^{\mu} \sigma^{\nu}\right]=2 g^{\mu \nu}, \\
& \operatorname{Tr}\left[\sigma^{\mu} \bar{\sigma}^{\nu} \sigma^{\rho} \bar{\sigma}^{\kappa}\right]=2\left(g^{\mu \nu} g^{\rho \kappa}-g^{\mu \rho} g^{\nu \kappa}+g^{\mu \kappa} g^{\nu \rho}+i \epsilon^{\mu \nu \rho \kappa}\right), \\
& \operatorname{Tr}\left[\bar{\sigma}^{\mu} \sigma^{\nu} \bar{\sigma}^{\rho} \sigma^{\kappa}\right]=2\left(g^{\mu \nu} g^{\rho \kappa}-g^{\mu \rho} g^{\nu \kappa}+g^{\mu \kappa} g^{\nu \rho}-i \epsilon^{\mu \nu \rho \kappa}\right) .
\end{aligned}
$$

Traces involving an odd number of $\sigma$ and $\bar{\sigma}$ matrices cannot arise, since there is no way to connect the spinor indices consistently. Additional identities involving $\sigma^{\mu \nu}$ and $\bar{\sigma}^{\mu \nu}$ can be found in Ref. [1].

Finally, we examine some useful identities involving bilinear spinor quantities. Although the two-component spinor fields appearing in these lectures are anticommuting, one also may encounter commuting twocomponent spinor wave functions. Thus, it is convenient to denote an arbitrary two-component spinor by $z_{i}$, and a sign factor, $(-1)^{A}=+1[-1]$, for commuting [anticommuting] spinors, respectively. Then, the following identities hold:

$$
\begin{aligned}
& z_{1} z_{2}=-(-1)^{A} z_{2} z_{1} \\
& z_{1}^{\dagger} z_{2}^{\dagger}=-(-1)^{A} z_{2}^{\dagger} z_{1}^{\dagger} \\
& z_{1} \sigma^{\mu} z_{2}^{\dagger}=(-1)^{A} z_{2}^{\dagger} \bar{\sigma}^{\mu} z_{1} \\
& z_{1} \sigma^{\mu} \bar{\sigma}^{\nu} z_{2}=-(-1)^{A} z_{2} \sigma^{\nu} \bar{\sigma}^{\mu} z_{1} \\
& z_{1}^{\dagger} \bar{\sigma}^{\mu} \sigma^{\nu} z_{2}^{\dagger}=-(-1)^{A} z_{2}^{\dagger} \bar{\sigma}^{\nu} \sigma^{\mu} z_{1}^{\dagger} \\
& z_{1}^{\dagger} \bar{\sigma}^{\mu} \sigma^{\rho} \bar{\sigma}^{\nu} z_{2}=(-1)^{A} z_{2} \sigma^{\nu} \bar{\sigma}^{\rho} \sigma^{\mu} z_{1}^{\dagger} .
\end{aligned}
$$

In many cases, it is convenient to rewrite a product of two bilinear spinor quantities in terms of products in which the individual spinors appear in a 
different order. Below, we provide five different Fierz identities, which are valid for both commuting and anticommuting spinors,

$$
\begin{aligned}
\left(z_{1} z_{2}\right)\left(z_{3} z_{4}\right) & =-\left(z_{1} z_{3}\right)\left(z_{4} z_{2}\right)-\left(z_{1} z_{4}\right)\left(z_{2} z_{3}\right), \\
\left(z_{1}^{\dagger} z_{2}^{\dagger}\right)\left(z_{3}^{\dagger} z_{4}^{\dagger}\right) & =-\left(z_{1}^{\dagger} z_{3}^{\dagger}\right)\left(z_{4}^{\dagger} z_{2}^{\dagger}\right)-\left(z_{1}^{\dagger} z_{4}^{\dagger}\right)\left(z_{2}^{\dagger} z_{3}^{\dagger}\right), \\
\left(z_{1} \sigma^{\mu} z_{2}^{\dagger}\right)\left(z_{3}^{\dagger} \bar{\sigma}_{\mu} z_{4}\right) & =-2\left(z_{1} z_{4}\right)\left(z_{2}^{\dagger} z_{3}^{\dagger}\right), \\
\left(z_{1}^{\dagger} \bar{\sigma}^{\mu} z_{2}\right)\left(z_{3}^{\dagger} \bar{\sigma}_{\mu} z_{4}\right) & =2\left(z_{1}^{\dagger} z_{3}^{\dagger}\right)\left(z_{4} z_{2}\right), \\
\left(z_{1} \sigma^{\mu} z_{2}^{\dagger}\right)\left(z_{3} \sigma_{\mu} z_{4}^{\dagger}\right) & =2\left(z_{1} z_{3}\right)\left(z_{4}^{\dagger} z_{2}^{\dagger}\right) .
\end{aligned}
$$

An exhaustive list of Fierz identities can be found in Appendix B of Ref. [1].

\subsubsection{Free field theories of two-component fermions}

The $\left(\frac{1}{2}, 0\right)$ spinor field $\xi_{\alpha}(x)$ describes a neutral Majorana fermion. The free-field Lagrangian is:

$$
\mathscr{L}=i \xi^{\dagger} \bar{\sigma}^{\mu} \partial_{\mu} \xi-\frac{1}{2} m\left(\xi \xi+\xi^{\dagger} \xi^{\dagger}\right),
$$

which is hermitian up to a total divergence since we can rewrite the above Lagrangian as

$$
\mathscr{L}=\frac{1}{2} i \xi^{\dagger} \bar{\sigma}^{\mu} \stackrel{\leftrightarrow}{\partial}_{\mu} \xi-\frac{1}{2} m\left(\xi \xi+\xi^{\dagger} \xi^{\dagger}\right)+\text { total divergence, }
$$

where $\xi^{\dagger} \bar{\sigma}^{\mu} \stackrel{\leftrightarrow}{\partial}_{\mu} \xi \equiv \xi^{\dagger} \bar{\sigma}^{\mu}\left(\partial_{\mu} \xi\right)-\left(\partial_{\mu} \xi\right)^{\dagger} \bar{\sigma}^{\mu} \xi$.

Generalizing to a multiplet of two-component fermion fields, $\hat{\xi}_{\alpha i}(x)$, labeled by flavor index $i$, the free Lagrangian is

$$
\mathscr{L}=i \hat{\xi}^{\dagger} \bar{\sigma}^{\mu} \partial_{\mu} \hat{\xi}_{i}-\frac{1}{2} M^{i j} \hat{\xi}_{i} \hat{\xi}_{j}-\frac{1}{2} M_{i j} \hat{\xi}^{\dagger i} \hat{\xi}^{\dagger j},
$$

where hermiticity implies that $M_{i j} \equiv\left(M^{i j}\right)^{*}$ is a complex symmetric matrix. To identify the physical fermion fields, we express the so-called interaction eigenstate fields, $\hat{\xi}_{\alpha i}(x)$, in terms of mass-eigenstate fields

$$
\xi(x)=\Omega^{-1} \hat{\xi}(x),
$$

where $\Omega$ is unitary and chosen such that

$$
\Omega^{\top} M \Omega=\boldsymbol{m}=\operatorname{diag}\left(m_{1}, m_{2}, \ldots\right),
$$

where the $m_{i}$ are non-negative real numbers. In linear algebra, this is called the Takagi diagonalization of a complex symmetric matrix $M[51,52] .^{5}$ To compute the values of the diagonal elements of $\boldsymbol{m}$, we note that

$$
\Omega^{\top} M M^{\dagger} \Omega^{*}=\boldsymbol{m}^{2} \text {. }
$$

Since $M M^{\dagger}$ is hermitian, it can be diagonalized by a unitary matrix. Thus, the $m_{i}$ of the Takagi diagonalization are the non-negative square-roots of the eigenvalues of $M M^{\dagger}$. In terms of the mass eigenstate fields,

$$
\mathscr{L}=i \xi^{\dagger} \bar{\sigma}^{\mu} \partial_{\mu} \xi_{i}-\frac{1}{2} m_{i}\left(\xi_{i} \xi_{i}+\xi^{\dagger i} \xi^{\dagger i}\right) .
$$

${ }^{5}$ Subsequently, it was recognized in Refs. $[53,54]$ that the Takagi diagonalization was first established for nonsingular complex symmetric matrices by Autonne [55]. 
Example 1 (The Seesaw Mechanism [56-60]).

The seesaw Lagrangian for the two-component fermions $\psi_{1}$ and $\psi_{2}$ is

$$
\mathscr{L}=i\left(\psi^{\dagger 1} \bar{\sigma}^{\mu} \partial_{\mu} \psi_{1}+\psi^{\dagger 2} \bar{\sigma}^{\mu} \partial_{\mu} \psi_{2}\right)-M^{i j} \psi_{i} \psi_{j}-M_{i j} \psi^{\dagger i} \psi^{\dagger j},
$$

where

$$
M^{i j}=\left(\begin{array}{cc}
0 & m_{D} \\
m_{D} & M
\end{array}\right),
$$

and (without loss of generality) $m_{D}$ and $M$ are real and positive. The Takagi diagonalization of this matrix is

$$
\Omega^{T} M \Omega=M_{D},
$$

where

$$
\Omega=\left(\begin{array}{rr}
i \cos \theta & \sin \theta \\
-i \sin \theta & \cos \theta
\end{array}\right), \quad M_{D}=\left(\begin{array}{cc}
m_{-} & 0 \\
0 & m_{+}
\end{array}\right),
$$

with

$$
m_{ \pm}=\frac{1}{2}\left[\sqrt{M^{2}+4 m_{D}^{2}} \pm M\right]
$$

and

$$
\sin 2 \theta=\frac{2 m_{D}}{\sqrt{M^{2}+4 m_{D}^{2}}} .
$$

If $M \gg m_{D}$, then the corresponding fermion masses are $m_{-} \simeq m_{D}^{2} / M$ and $m_{+} \simeq M$, with $\sin \theta \simeq m_{D} / M$. The mass eigenstates, $\chi_{i}$ are given by $\psi_{i}=\Omega_{i}{ }^{j} \chi_{j}$; to leading order in $m_{d} / M$,

$$
i \chi_{1} \simeq \psi_{1}-\frac{m_{D}}{M} \psi_{2}, \quad \chi_{2} \simeq \psi_{2}+\frac{m_{D}}{M} \psi_{1} .
$$

Indeed, one can check that:

$$
\begin{aligned}
\frac{1}{2} m_{D}\left(\psi_{1} \psi_{2}+\psi_{2} \psi_{1}\right)+\frac{1}{2} M \psi_{2} \psi_{2} & + \text { h.c. } \\
& \simeq \frac{1}{2}\left[\frac{m_{D}^{2}}{M} \chi_{1} \chi_{1}+M \chi_{2} \chi_{2}+\text { h.c. }\right],
\end{aligned}
$$

which corresponds to a theory of two Majorana fermions - one very light and one very heavy (the seesaw).

In any theory containing a multiplet of fields, one can check for the existence of global symmetries. The simplest case is a theory of a pair of two-component $\left(\frac{1}{2}, 0\right)$ fermion fields $\chi$ and $\eta$, with the free-field Lagrangian,

$$
\mathscr{L}=i \chi^{\dagger} \bar{\sigma}^{\mu} \partial_{\mu} \chi+i \eta^{\dagger} \bar{\sigma}^{\mu} \partial_{\mu} \eta-m\left(\chi \eta+\chi^{\dagger} \eta^{\dagger}\right) .
$$


The Lagrangian given in eq. (2.83) possesses a U(1) global symmetry, $\chi \rightarrow e^{i \theta} \chi$ and $\eta \rightarrow e^{-i \theta} \eta$. That is, $\chi$ and $\eta$ are oppositely charged. The corresponding mass matrix is

$$
M=\left(\begin{array}{cc}
0 & m \\
m & 0
\end{array}\right)
$$

Performing the Takagi diagonalization yields two degenerate twocomponent fermions of mass $m$. However, the corresponding masseigenstates are not eigenstates of charge. ${ }^{6}$ Together, $\chi$ and $\eta^{\dagger}$ constitute a single (four-component) Dirac fermion.

More generally, consider a collection of charged Dirac fermions represented by pairs of two-component interaction eigenstate fields $\hat{\chi}_{\alpha i}(x), \hat{\eta}_{\alpha}^{i}(x)$, with

$$
\mathscr{L}=i \hat{\chi}^{\dagger i} \bar{\sigma}^{\mu} \partial_{\mu} \hat{\chi}_{i}+i \hat{\eta}_{i}^{\dagger} \bar{\sigma}^{\mu} \partial_{\mu} \hat{\eta}^{i}-M^{i}{ }_{j} \hat{\chi}_{i} \hat{\eta}^{j}-M_{i}{ }^{j} \hat{\chi}^{\dagger i} \hat{\eta}_{j}^{\dagger},
$$

where $M$ is a complex matrix with matrix elements denoted by $M^{i}{ }_{j}$ (note the placement of the flavor indices $i$ and $j$ ), and $M_{i}{ }^{j} \equiv\left(M^{i}{ }_{j}\right)^{*}$.

We denote the mass eigenstate fields by $\chi_{i}$ and $\eta^{i}$ and the unitary matrices $L$ and $R$, such that $\hat{\chi}_{i}=L_{i}{ }^{k} \chi_{k}$ and $\hat{\eta}^{i}=R^{i}{ }_{k} \eta^{k}$, and

$$
L^{\top} M R=\boldsymbol{m}=\operatorname{diag}\left(m_{1}, m_{2}, \ldots\right),
$$

where the $m_{i}$ are non-negative real numbers. This is the singular value decomposition of a complex matrix (see, e.g., Refs. [53, 54]). Noting that

$$
R^{\dagger}\left(M^{\dagger} M\right) R=\boldsymbol{m}^{2},
$$

the diagonal elements of $\boldsymbol{m}$ are the non-negative square roots of the corresponding eigenvalues of $M^{\dagger} M$. In terms of the mass eigenstate fields,

$$
\mathscr{L}=i \chi^{\dagger i} \bar{\sigma}^{\mu} \partial_{\mu} \chi_{i}+i \eta_{i}^{\dagger} \bar{\sigma}^{\mu} \partial_{\mu} \eta^{i}-m_{i}\left(\chi_{i} \eta^{i}+\chi^{\dagger i} \eta_{i}^{\dagger}\right) \text {. }
$$

\subsubsection{Fermion-scalar interactions}

The most general set of interactions with the scalars of the theory $\hat{\phi}_{I}$ are then given by:

$$
\mathscr{L}_{\text {int }}=-\frac{1}{2} \hat{Y}^{I j k} \hat{\phi}_{I} \hat{\psi}_{j} \hat{\psi}_{k}-\frac{1}{2} \hat{Y}_{I j k} \hat{\phi}^{I} \hat{\psi}^{\dagger j} \hat{\psi}^{\dagger k},
$$

where $\hat{Y}_{I j k} \equiv\left(\hat{Y}^{I j k}\right)^{*}$ and $\hat{\phi}^{I} \equiv\left(\hat{\phi}_{I}\right)^{*}$. The flavor index $I$ runs over a collection of real scalar fields $\hat{\varphi}_{i}$ and pairs of complex scalar fields $\hat{\Phi}_{j}$ and

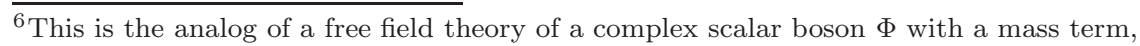
$\mathscr{L}_{\text {mass }}=-m^{2}|\Phi|^{2}$. Writing $\Phi=\left(\phi_{1}+i \phi_{2}\right) / \sqrt{2}$, we can write Lagrangian in terms of $\phi_{1}$ and $\phi_{2}$ with a diagonal mass term. But, $\phi_{1}$ and $\phi_{2}$ do not correspond to states of definite charge. 
$\hat{\Phi}^{j} \equiv\left(\hat{\Phi}_{j}\right)^{*}$ (where a complex field and its conjugate are counted separately). The Yukawa couplings $\hat{Y}^{I j k}$ are symmetric under interchange of $j$ and $k$.

The mass-eigenstate basis $\psi$ is related to the interaction-eigenstate basis $\hat{\psi}$ by a unitary transformation,

$$
\hat{\psi} \equiv\left(\begin{array}{c}
\hat{\xi} \\
\hat{\chi} \\
\hat{\eta}
\end{array}\right)=U \psi \equiv\left(\begin{array}{ccc}
\Omega & 0 & 0 \\
0 & L & 0 \\
0 & 0 & R
\end{array}\right)\left(\begin{array}{l}
\xi \\
\chi \\
\eta
\end{array}\right),
$$

where $\Omega, L$, and $R$ are constructed as described previously. Likewise a unitary transformation yields the scalar mass-eigenstates via $\hat{\phi}=V \phi$. Thus, in terms of mass-eigenstate fields:

$$
\mathscr{L}_{\text {int }}=-\frac{1}{2} Y^{I j k} \phi_{I} \psi_{j} \psi_{k}-\frac{1}{2} Y_{I j k} \phi^{I} \psi^{\dagger j} \psi^{\dagger k},
$$

where $Y^{I j k}=V_{J}{ }^{I} U_{m}{ }^{j} U_{n}{ }^{k} \hat{Y}^{J m n}$.

\subsubsection{Fermion-gauge boson interactions}

In the gauge-interaction basis for the two-component fermions the corresponding interaction Lagrangian is given by

$$
\mathscr{L}_{\text {int }}=-g_{a} A_{a}^{\mu} \hat{\psi}^{\dagger i} \bar{\sigma}_{\mu}\left(\boldsymbol{T}^{a}\right)_{i}{ }^{j} \hat{\psi}_{j}
$$

where the index $a$ labels the (real or complex) vector bosons $A_{a}^{\mu}$ and is summed over. If the gauge symmetry is unbroken, then the index $a$ runs over the adjoint representation of the gauge group, and the $\left(\boldsymbol{T}^{a}\right)_{i}{ }^{j}$ are hermitian representation matrices ${ }^{7}$ of the gauge group acting on the fermions. There is a separate coupling $g_{a}$ for each simple group or $\mathrm{U}(1)$ factor of the gauge group $\mathrm{G}$.

In the case of spontaneously broken gauge theories, one must diagonalize the vector boson squared-mass matrix. The form of eq. (2.92) still applies where $A_{\mu}^{a}$ are gauge boson fields of definite mass, although in this case for a fixed value of $a$, the product $g_{a} \boldsymbol{T}^{a}$ is some linear combination of the original $g_{a} \boldsymbol{T}^{a}$ of the unbroken theory. That is, the hermitian matrix gauge field $\left(A_{\mu}\right)_{i}{ }^{j} \equiv A_{\mu}^{a}\left(\boldsymbol{T}^{\boldsymbol{a}}\right)_{i}{ }^{j}$ appearing in eq. (2.92) can always be reexpressed in terms of the physical mass eigenstate gauge boson fields. If an

${ }^{7}$ For a $U(1)$ gauge group, the $\boldsymbol{T}^{a}$ are replaced by real numbers corresponding to the $\mathrm{U}(1)$ charges of the $\left(\frac{1}{2}, 0\right)$ fermions. 
unbroken $\mathrm{U}(1)$ symmetry exists, then the physical gauge bosons will also be eigenstates of the conserved U(1)-charge. ${ }^{8}$

In terms of mass-eigenstate fermion fields,

$$
\mathscr{L}_{\text {int }}=-A_{a}^{\mu} \psi^{\dagger i} \bar{\sigma}_{\mu}\left(G^{a}\right)_{i}{ }^{j} \psi_{j}
$$

where $G^{a}=g_{a} U^{\dagger} \boldsymbol{T}^{a} U$ (no sum over $a$ ).

The case of gauge interactions of charged Dirac fermions can be treated as follows. Consider pairs of $\left(\frac{1}{2}, 0\right)$ interaction-eigenstate fermions $\hat{\chi}_{i}$ and $\hat{\eta}^{i}$ that transform as conjugate representations of the gauge group (hence the difference in the flavor index heights). The Lagrangian for the gauge interactions of Dirac fermions can be written in the form:

$$
\mathscr{L}_{\text {int }}=-g_{a} A_{a}^{\mu} \hat{\chi}^{\dagger i} \bar{\sigma}_{\mu}\left(\boldsymbol{T}^{a}\right)_{i}{ }^{j} \hat{\chi}_{j}+g_{a} A_{a}^{\mu} \hat{\eta}_{i}^{\dagger} \bar{\sigma}_{\mu}\left(\boldsymbol{T}^{a}\right)_{j}{ }_{j} \hat{\eta}^{j},
$$

where the $A_{\mu}^{a}$ are gauge boson mass-eigenstate fields. Here we have used the fact that if $\left(\boldsymbol{T}^{a}\right)_{i}{ }^{j}$ are the representation matrices for the $\hat{\chi}_{i}$, then the $\hat{\eta}^{i}$ transform in the complex conjugate representation with generator matrices $-\left(\boldsymbol{T}^{a}\right)^{*}=-\left(\boldsymbol{T}^{a}\right)^{T}$. In terms of mass-eigenstate fermion fields,

$$
\mathscr{L}_{\mathrm{int}}=-A_{a}^{\mu}\left[\chi^{\dagger i} \bar{\sigma}_{\mu}\left(G_{L}^{a}\right)_{i}{ }^{j} \chi_{j}-\eta_{i}^{\dagger} \bar{\sigma}_{\mu}\left(G_{R}^{a}\right)_{j}{ }^{i} \eta^{j}\right],
$$

where $G_{L}^{a}=g_{a} L^{\dagger} \boldsymbol{T}^{a} L$ and $G_{R}^{a}=g_{a} R^{\dagger} \boldsymbol{T}^{a} R$ (no sum over $a$ ).

\subsection{Correspondence between the two-component and four- component spinor notations}

Most pedagogical treatments of calculations in particle physics employ fourcomponent Dirac spinor notation, which combines distinct irreducible representations of the Lorentz symmetry algebra. Parity-conserving theories such as QED and QCD and their Feynman rules are especially well-suited to four-component spinor notation. In light of the widespread familiarity with four-component spinor techniques, we provide in this section a translation between two-component and four-component spinor notation.

\footnotetext{
${ }^{8}$ In terms of the physical gauge boson fields, $A_{\mu}^{a} \boldsymbol{T}^{a}$ consists of a sum over real neutral gauge fields multiplied by hermitian generators, and complex charged gauge fields multiplied by non-hermitian generators. For example, in the electroweak Standard Model, $\mathrm{G}=\mathrm{SU}(2) \times \mathrm{U}(1)$ with gauge bosons and generators $W_{\mu}^{a}$ and $\boldsymbol{T}^{a}=\frac{1}{2} \tau^{a}$ for $\mathrm{SU}(2)$, and $B_{\mu}$ and $\boldsymbol{Y}$ for $\mathrm{U}(1)$, where the $\tau^{a}$ are the usual Pauli matrices. After diagonalizing the gauge boson squared-mass matrix,

$g W_{\mu}^{a} \boldsymbol{T}^{a}+g^{\prime} B_{\mu} \boldsymbol{Y}=\frac{g}{\sqrt{2}}\left(W_{\mu}^{+} \boldsymbol{T}^{+}+W_{\mu}^{-} \boldsymbol{T}^{-}\right)+\frac{g}{\cos \theta_{W}}\left(\boldsymbol{T}^{\mathbf{3}}-\boldsymbol{Q} \sin ^{2} \theta_{W}\right) Z_{\mu}+e \boldsymbol{Q} A_{\mu}$, where $\boldsymbol{Q}=\boldsymbol{T}^{\mathbf{3}}+\boldsymbol{Y}$ is the generator of the unbroken $\mathrm{U}(1)_{\mathrm{EM}}, \boldsymbol{T}^{ \pm} \equiv \boldsymbol{T}^{\mathbf{1}} \pm i \boldsymbol{T}^{\mathbf{2}}$, and $e=g \sin \theta_{W}=g^{\prime} \cos \theta_{W}$. The massive gauge boson charge-eigenstate fields of the broken theory consist of a charged massive gauge boson pair, $W^{ \pm} \equiv\left(W^{1} \mp i W^{2}\right) / \sqrt{2}$, a neutral massive gauge boson, $Z \equiv W^{3} \cos \theta_{W}-B \sin \theta_{W}$, and the massless photon, $A \equiv W^{3} \sin \theta_{W}+B \cos \theta_{W}$
} 


\subsubsection{From two-component to four-component spinor notation}

The correspondence between the two-component and four-component spinor language is most easily exhibited in the basis in which $\gamma_{5}$ is diagonal (this is called the chiral representation). Employing $2 \times 2$ matrix blocks, the gamma matrices are given by:

$$
\gamma^{\mu}=\left(\begin{array}{cc}
0 & \sigma_{\alpha \dot{\beta}}^{\mu} \\
\bar{\sigma}^{\mu \dot{\alpha} \beta} & 0
\end{array}\right), \quad \gamma_{5} \equiv i \gamma^{0} \gamma^{1} \gamma^{2} \gamma^{3}=\left(\begin{array}{cc}
-\delta_{\alpha}{ }^{\beta} & 0 \\
0 & \delta^{\dot{\alpha}} \dot{\beta}
\end{array}\right) .
$$

The chiral projections operators are

$$
\begin{aligned}
& P_{L} \equiv \frac{1}{2}\left(1-\gamma_{5}\right), \\
& P_{R} \equiv \frac{1}{2}\left(1+\gamma_{5}\right) .
\end{aligned}
$$

In addition, we identify the generators of the Lorentz group in the reducible $\left(\frac{1}{2}, 0\right) \oplus\left(0, \frac{1}{2}\right)$ representation $^{9}$

$$
\frac{1}{2} \Sigma^{\mu \nu} \equiv \frac{i}{4}\left[\gamma^{\mu}, \gamma^{\nu}\right]=\left(\begin{array}{cc}
\sigma^{\mu \nu}{ }^{\beta} & 0 \\
0 & \bar{\sigma}^{\mu \nu \dot{\alpha}} \dot{\beta}
\end{array}\right),
$$

where $\Sigma^{\mu \nu}$ satisfies the duality relation, $\gamma_{5} \Sigma^{\mu \nu}=\frac{1}{2} i \epsilon^{\mu \nu \rho \tau} \Sigma_{\rho \tau}$.

A four-component Dirac spinor field, $\Psi(x)$, is made up of two massdegenerate two-component spinor fields, $\chi_{\alpha}(x)$ and $\eta_{\alpha}(x)$ as follows:

$$
\Psi(x) \equiv\left(\begin{array}{c}
\chi_{\alpha}(x) \\
\eta^{\dagger} \dot{\alpha}(x)
\end{array}\right)
$$

Note that $P_{L}$ and $P_{R}$ project out the upper and lower components, respectively. The Dirac conjugate field $\bar{\Psi}$ and the charge conjugate field $\Psi^{c}$ are defined by

$$
\begin{gathered}
\bar{\Psi}(x) \equiv \Psi^{\dagger} A=\left(\eta^{\alpha}(x), \chi_{\dot{\alpha}}^{\dagger}(x)\right), \\
\Psi^{c}(x) \equiv C \bar{\Psi}^{\top}(x)=\left(\begin{array}{c}
\eta_{\alpha}(x) \\
\chi^{\dagger \dot{\alpha}}(x)
\end{array}\right),
\end{gathered}
$$

where the Dirac conjugation matrix $A$ and the charge conjugation matrix $C$ satisfy

$$
A \gamma^{\mu} A^{-1}=\gamma^{\mu \dagger}, \quad \quad C^{-1} \gamma^{\mu} C=-\gamma^{\mu \top} .
$$

It is conventional to impose two additional conditions:

$$
\Psi=A^{-1} \bar{\Psi}^{\dagger}, \quad\left(\Psi^{c}\right)^{c}=\Psi .
$$

${ }^{9}$ In most textbooks, $\Sigma^{\mu \nu}$ is called $\sigma^{\mu \nu}$. Here, we use the former symbol so that there is no confusion with the two-component definition of $\sigma^{\mu \nu}$. 
The first of these conditions together with eq. (2.101) is equivalent to the statement that $\bar{\Psi} \Psi$ is hermitian. The second condition corresponds to the statement that the (discrete) charge conjugation transformation applied twice is equal to the identity operator. It then follows that

$$
A^{\dagger}=A, \quad C^{\top}=-C, \quad(A C)^{-1}=(A C)^{*} .
$$

In the chiral representation, $A$ and $C$ are explicitly given by

$$
A=\left(\begin{array}{cc}
0 & \delta^{\dot{\alpha}} \dot{\beta} \\
\delta_{\alpha}{ }^{\beta} & 0
\end{array}\right), \quad C=\left(\begin{array}{cc}
\epsilon_{\alpha \beta} & 0 \\
0 & \epsilon^{\dot{\alpha} \dot{\beta}}
\end{array}\right) .
$$

Note the numerical equalities, $A=\gamma^{0}$ and $C=i \gamma^{0} \gamma^{2}$, although these identifications do not respect the structure of the undotted and dotted indices specified above.

Finally, we note the following results, which are easily derived:

$$
\begin{aligned}
& A \Gamma A^{-1}=\eta_{\Gamma}^{A} \Gamma^{\dagger}, \quad \eta_{\Gamma}^{A}= \begin{cases}+1, & \text { for } \Gamma=\mathbb{1}, \gamma^{\mu}, \gamma^{\mu} \gamma_{5}, \Sigma^{\mu \nu}, \\
-1, & \text { for } \Gamma=\gamma_{5}, \Sigma^{\mu \nu} \gamma_{5},\end{cases} \\
& C^{-1} \Gamma C=\eta_{\Gamma}^{C} \Gamma^{\top},
\end{aligned}
$$

\subsubsection{Four-component spinor bilinear covariants}

The Dirac bilinear covariants are quantities that are quadratic in the Dirac spinor fields and transform irreducibly as Lorentz tensors. These may be constructed from the corresponding quantities that are quadratic in the two-component spinors. To construct a translation table between the twocomponent spinor and four-component spinor forms of the bilinear covariants, we first define two Dirac spinor fields,

$$
\Psi_{1}(x) \equiv\left(\begin{array}{c}
\chi_{1}(x) \\
\eta_{1}^{\dagger}(x)
\end{array}\right), \quad \Psi_{2}(x) \equiv\left(\begin{array}{c}
\chi_{2}(x) \\
\eta_{2}^{\dagger}(x)
\end{array}\right),
$$

where spinor indices have been suppressed. It follows that,

$$
\begin{aligned}
& \bar{\Psi}_{1} \Psi_{2}=\eta_{1} \chi_{2}+\chi_{1}^{\dagger} \eta_{2}^{\dagger}, \\
& \bar{\Psi}_{1} \gamma_{5} \Psi_{2}=-\eta_{1} \chi_{2}+\chi_{1}^{\dagger} \eta_{2}^{\dagger}, \\
& \bar{\Psi}_{1} \gamma^{\mu} \Psi_{2}=\chi_{1}^{\dagger} \bar{\sigma}^{\mu} \chi_{2}+\eta_{1} \sigma^{\mu} \eta_{2}^{\dagger}, \\
& \bar{\Psi}_{1} \gamma^{\mu} \gamma_{5} \Psi_{2}=-\chi_{1}^{\dagger} \bar{\sigma}^{\mu} \chi_{2}+\eta_{1} \sigma^{\mu} \eta_{2}^{\dagger}, \\
& \bar{\Psi}_{1} \Sigma^{\mu \nu} \Psi_{2}=2\left(\eta_{1} \sigma^{\mu \nu} \chi_{2}+\chi_{1}^{\dagger} \bar{\sigma}^{\mu \nu} \eta_{2}^{\dagger}\right), \\
& \bar{\Psi}_{1} \Sigma^{\mu \nu} \gamma_{5} \Psi_{2}=-2\left(\eta_{1} \sigma^{\mu \nu} \chi_{2}-\chi_{1}^{\dagger} \bar{\sigma}^{\mu \nu} \eta_{2}^{\dagger}\right) .
\end{aligned}
$$

The above results can be used to to obtain the translations given in Table 1 . 
Table 1.: Relating the Dirac bilinear covariants written in terms of fourcomponent Dirac spinor fields to the corresponding quantities expressed in terms of two-component spinor fields using the notation of eq. (2.100). These results apply to both commuting and anticommuting spinors. In the latter case, one may alternatively write $\bar{\Psi}_{1} \gamma^{\mu} P_{R} \Psi_{2}=-\eta_{2}^{\dagger} \bar{\sigma}^{\mu} \eta_{1}$, etc. [cf. eq. (2.59)].

\begin{tabular}{|l|l|}
\hline $\bar{\Psi}_{1} P_{L} \Psi_{2}=\eta_{1} \chi_{2}$ & $\bar{\Psi}_{1}^{c} P_{L} \Psi_{2}^{c}=\chi_{1} \eta_{2}$ \\
$\bar{\Psi}_{1} P_{R} \Psi_{2}=\chi_{1}^{\dagger} \eta_{2}^{\dagger}$ & $\bar{\Psi}_{1}^{c} P_{R} \Psi_{2}^{c}=\eta_{1}^{\dagger} \chi_{2}^{\dagger}$ \\
$\bar{\Psi}_{1}^{c} P_{L} \Psi_{2}=\chi_{1} \chi_{2}$ & $\bar{\Psi}_{1} P_{L} \Psi_{2}^{c}=\eta_{1} \eta_{2}$ \\
$\bar{\Psi}_{1} P_{R} \Psi_{2}^{c}=\chi_{1}^{\dagger} \chi_{2}^{\dagger}$ & $\bar{\Psi}_{1}^{c} P_{R} \Psi_{2}=\eta_{1}^{\dagger} \eta_{2}^{\dagger}$ \\
$\bar{\Psi}_{1} \gamma^{\mu} P_{L} \Psi_{2}=\chi_{1}^{\dagger} \bar{\sigma}^{\mu} \chi_{2}$ & $\bar{\Psi}_{1}^{c} \gamma^{\mu} P_{L} \Psi_{2}^{c}=\eta_{1}^{\dagger} \bar{\sigma}^{\mu} \eta_{2}$ \\
$\bar{\Psi}_{1}^{c} \gamma^{\mu} P_{R} \Psi_{2}^{c}=\chi_{1} \sigma^{\mu} \chi_{2}^{\dagger}$ & $\bar{\Psi}_{1} \gamma^{\mu} P_{R} \Psi_{2}=\eta_{1} \sigma^{\mu} \eta_{2}^{\dagger}$ \\
$\bar{\Psi}_{1} \Sigma^{\mu \nu} P_{L} \Psi_{2}=2 \eta_{1} \sigma^{\mu \nu} \chi_{2}$ & $\bar{\Psi}_{1}^{c} \Sigma^{\mu \nu} P_{L} \Psi_{2}^{c}=2 \chi_{1} \sigma^{\mu \nu} \eta_{2}$ \\
$\bar{\Psi}_{1} \Sigma^{\mu \nu} P_{R} \Psi_{2}=2 \chi_{1}^{\dagger} \bar{\sigma}^{\mu \nu} \eta_{2}^{\dagger}$ & $\bar{\Psi}_{1}^{c} \Sigma^{\mu \nu} P_{R} \Psi_{2}^{c}=2 \eta_{1}^{\dagger} \bar{\sigma}^{\mu \nu} \chi_{2}^{\dagger}$ \\
\hline
\end{tabular}

When $\Psi_{2}=\Psi_{1}$, the bilinear covariants listed in eqs. (2.110)-(2.115) are either hermitian or anti-hermitian. Using eq. (2.107), it follows that $\bar{\Psi} \Gamma \Psi$ is hermitian for $\Gamma=\mathbb{1}_{4 \times 4}, i \gamma_{5}, \gamma^{\mu}, \gamma^{\mu} \gamma_{5}, \Sigma^{\mu \nu}$, and $i \Sigma^{\mu \nu} \gamma_{5}$.

One can also define Majorana bilinear covariants. A four-component Majorana fermion field is defined by the condition,

$$
\Psi_{M}(x)=\Psi_{M}^{c}(x)=C \bar{\Psi}_{M}^{T}(x)=\left(\begin{array}{c}
\xi_{\alpha}(x) \\
\xi^{\dot{\alpha} \dagger}(x)
\end{array}\right) .
$$

Eqs. (2.110)-(2.115) and the results of Table 1 may also be applied to fourcomponent Majorana spinors, $\Psi_{M 1}$ and $\Psi_{M 2}$, by setting $\xi_{1} \equiv \chi_{1}=\eta_{1}$, and $\xi_{2} \equiv \chi_{2}=\eta_{2}$, respectively. This implements the Majorana condition given in eq. (2.116) and imposes additional restrictions on the Majorana bilinear covariants. In particular, the anticommuting Majorana four-component fermion fields satisfy the following additional identities,

$$
\begin{aligned}
\bar{\Psi}_{M 1} \Psi_{M 2} & =\bar{\Psi}_{M 2} \Psi_{M 1}, \\
\bar{\Psi}_{M 1} \gamma_{5} \Psi_{M 2} & =\bar{\Psi}_{M 2} \gamma_{5} \Psi_{M 1}, \\
\bar{\Psi}_{M 1} \gamma^{\mu} \Psi_{M 2} & =-\bar{\Psi}_{M 2} \gamma^{\mu} \Psi_{M 1}, \\
\bar{\Psi}_{M 1} \gamma^{\mu} \gamma_{5} \Psi_{M 2} & =\bar{\Psi}_{M 2} \gamma^{\mu} \gamma_{5} \Psi_{M 1}, \\
\bar{\Psi}_{M 1} \Sigma^{\mu \nu} \Psi_{M 2} & =-\bar{\Psi}_{M 2} \Sigma^{\mu \nu} \Psi_{M 1}, \\
\bar{\Psi}_{M 1} \Sigma^{\mu \nu} \gamma_{5} \Psi_{M 2} & =-\bar{\Psi}_{M 2} \Sigma^{\mu \nu} \gamma_{5} \Psi_{M 1} .
\end{aligned}
$$


If $\Psi_{M 1}=\Psi_{M 2} \equiv \Psi_{M}$, then eqs. (2.117)-(2.122) yield

$$
\bar{\Psi}_{M} \gamma^{\mu} \Psi_{M}=\bar{\Psi}_{M} \Sigma^{\mu \nu} \Psi_{M}=\bar{\Psi}_{M} \Sigma^{\mu \nu} \gamma_{5} \Psi_{M}=0
$$

One additional useful result for Majorana fermion fields is:

$$
\bar{\Psi}_{M 1} \gamma^{\mu} P_{L} \Psi_{M 2}=-\bar{\Psi}_{M 2} \gamma^{\mu} P_{R} \Psi_{M 1} \text {. }
$$

\subsection{Feynman Rules for Dirac and Majorana fermions}

The application of four-component fermion techniques in parity-violating theories is straightforward for processes involving Dirac fermions. However, the inclusion of Majorana fermions involves some subtleties that require elucidation. In light of the widespread familiarity with four-component spinor techniques, we shall develop four-component fermion Feynman rules that treat Dirac and Majorana fermions on equal footing $[1,61-63] .{ }^{10}$

Consider first the Feynman rule for the four-component fermion propagator. Virtual Dirac fermion lines can either correspond to $\Psi$ or $\Psi^{c}$. Here, there is no ambiguity in the propagator Feynman rule, since for free Dirac fermion fields,

$$
\langle 0|T[\Psi(x) \bar{\Psi}(y)]| 0\rangle=\left\langle 0\left|T\left[\Psi^{c}(x) \overline{\Psi^{c}}(y)\right]\right| 0\right\rangle,
$$

so that the Feynman rules for the propagator of a $\Psi$ and $\Psi^{c}$ line, exhibited below, are identical. The same rule also applies to a four-component Majorana fermion $\Psi_{M}$.

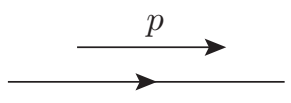

$$
\frac{i(\not p+m)}{p^{2}-m^{2}+i \epsilon}
$$

Consider next a set of neutral Majorana fermions $\Psi_{M i}$ and charged Dirac fermions $\Psi_{i}$,

$$
\Psi_{M i}=\left(\begin{array}{c}
\xi_{i} \\
\xi_{i}^{\dagger}
\end{array}\right), \quad \Psi_{i}=\left(\begin{array}{c}
\chi_{i} \\
\eta_{i}^{\dagger}
\end{array}\right),
$$

interacting with a neutral scalar $\phi$ or vector boson $A_{\mu}$. The interaction Lagrangian in terms of two-component fermions is

$$
\begin{aligned}
\mathscr{L}_{\text {int }}= & -\frac{1}{2}\left(\lambda^{i j} \xi_{i} \xi_{j}+\lambda_{i j} \xi^{\dagger i} \xi^{\dagger j}\right) \phi-\left(\kappa^{i}{ }_{j} \chi_{i} \eta^{j}+\kappa_{i}{ }^{j} \chi^{\dagger i} \eta_{j}^{\dagger}\right) \phi \\
& -G_{i}{ }^{j} \xi^{\dagger i} \bar{\sigma}^{\mu} \xi_{j} A_{\mu}-\left[\left(G_{L}\right){ }_{i}{ }^{j} \chi^{\dagger i} \bar{\sigma}^{\mu} \chi_{j}+\left(G_{R}\right)_{i}{ }^{j} \eta^{\dagger i} \bar{\sigma}^{\mu} \eta_{j}\right] A_{\mu},(2.127)
\end{aligned}
$$

where $\lambda$ is a complex symmetric matrix with $\lambda^{i j} \equiv \lambda_{i j}^{*}, \kappa$ is an arbitrary complex matrix with $\kappa_{i}{ }^{j} \equiv\left(\kappa^{i}{ }_{j}\right)^{*}$, and $G, G_{L}$ and $G_{R}$ are hermitian matrices. Converting to four-component spinor notation (see Problem 1), the resulting Feynman rules are shown below.

${ }^{10}$ For a comprehensive set of two-component fermion Feynman rules, see Ref. [1]. 

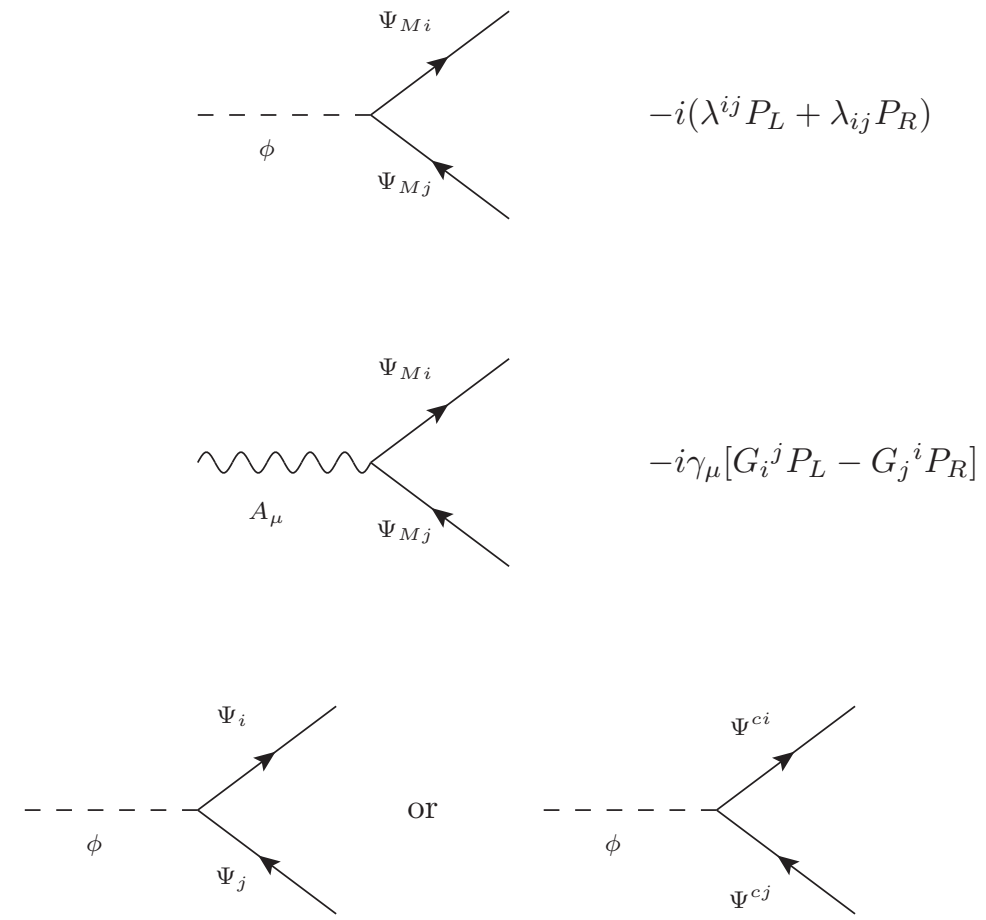

or

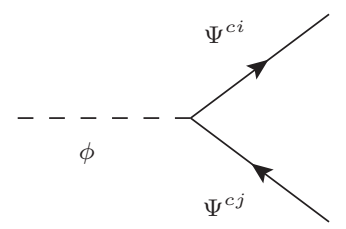

$-i\left(\kappa_{j}^{i} P_{L}+\kappa_{j}{ }^{i} P_{R}\right)$

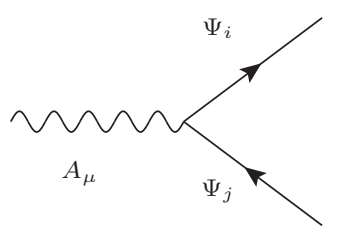

or

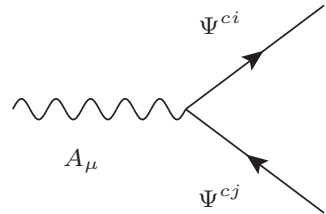

$$
-i \gamma_{\mu}\left[\left(G_{L}\right)_{i}{ }^{j} P_{L}+\left(G_{R}\right)_{i}{ }^{j} P_{R}\right]
$$

or

$i \gamma_{\mu}\left[\left(G_{L}\right)_{i}{ }^{j} P_{L}+\left(G_{R}\right)_{i}{ }^{j} P_{R}\right]$ 
The arrows on the Dirac fermion lines depict the flow of the conserved charge. A Majorana fermion is self-conjugate, so its arrow simply reflects the structure of $\mathscr{L}_{\text {int }}$; i.e., $\bar{\Psi}_{M}\left[\Psi_{M}\right]$ is represented by an arrow pointing out of [into] the vertex. The arrow directions determine the placement of the $u$ and $v$ spinors in an invariant amplitude.

For vertices involving Dirac fermions, one has a choice of either using the Dirac field or its charge conjugated field. The Feynman rules corresponding to these two choices are related, due to the following identity,

$$
\bar{\Psi}_{i}^{c} \Gamma \Psi_{j}^{c}=-\Psi_{i}^{T} C^{-1} \Gamma C \bar{\Psi}_{j}^{T}=\bar{\Psi}_{j} C \Gamma^{T} C^{-1} \Psi_{i}=\eta_{\Gamma}^{C} \bar{\Psi}_{j} \Gamma \Psi_{i},
$$

where we have used eq. (2.108). Note that the extra minus sign that arises in the penultimate step above is due to the anticommutativity of the fermion fields.

Next, consider the interaction of fermions with charged bosons $\Phi$ and $W$ (assumed to have charge equal to that of $\chi$ and $\eta^{\dagger}$ ). The corresponding interaction Lagrangian is given by:

$$
\begin{aligned}
\mathscr{L}_{\text {int }}= & -\Phi\left[\left(\kappa_{1}\right)^{i}{ }_{j} \xi_{i} \eta^{j}+\left(\kappa_{2}\right)_{i j} \xi^{\dagger i} \chi^{\dagger j}\right]-\Phi^{\dagger}\left[\left(\kappa_{2}\right)^{i j} \xi_{i} \chi_{j}+\left(\kappa_{1}\right)_{i}{ }^{j} \xi_{i}^{\dagger i} \eta_{j}^{\dagger}\right] \\
& -W_{\mu}\left[\left(G_{1}\right)_{j}{ }_{j} \chi^{\dagger j} \bar{\sigma}^{\mu} \xi_{i}-\left(G_{2}\right)_{i j} \xi^{\dagger i} \bar{\sigma}^{\mu} \eta^{j}\right] \\
& -W_{\mu}^{\dagger}\left[\left(G_{1}\right)_{i}{ }_{i} \xi^{\dagger i} \bar{\sigma}^{\mu} \chi_{j}-\left(G_{2}\right)^{i j} \eta_{j}^{\dagger} \bar{\sigma}^{\mu} \xi_{i}\right],
\end{aligned}
$$

where $\kappa_{1}, \kappa_{2}, G_{1}$ and $G_{2}$ are complex matrices. Converting to fourcomponent spinor notation, the corresponding Feynman rules are:

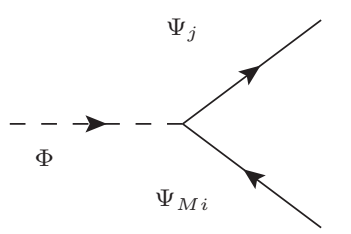

or
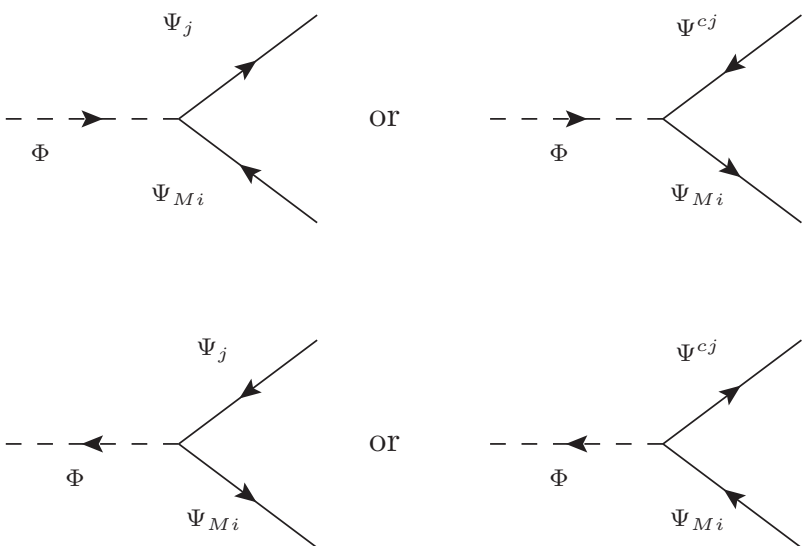

or

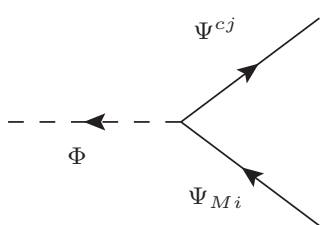

$-i\left(\kappa_{1}{ }^{i}{ }_{j} P_{L}+\kappa_{2 i j} P_{R}\right)$

$-i\left(\kappa_{2}^{i j} P_{L}+\kappa_{1 i}{ }^{j} P_{R}\right)$ 


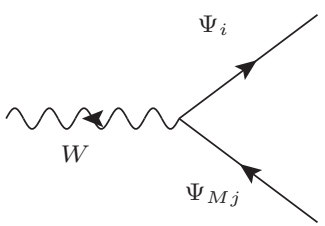

or
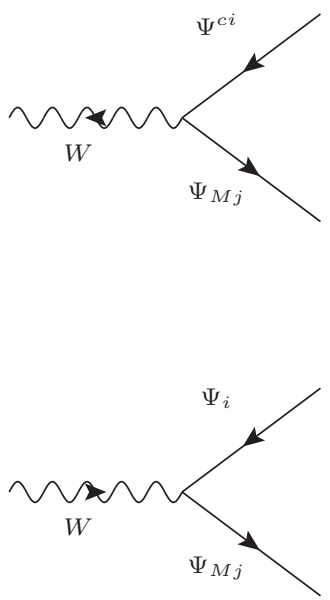

or

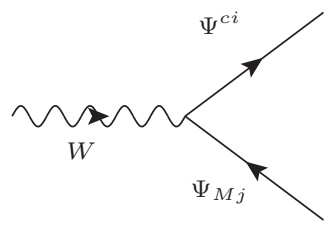

$-i \gamma^{\mu}\left(G_{1 i}{ }^{j} P_{L}-G_{2 j i} P_{R}\right)$

or

$i \gamma^{\mu}\left(G_{2 j i} P_{R}-G_{1 i}{ }^{j} P_{L}\right)$

$-i \gamma^{\mu}\left(G_{1}{ }_{j} P_{L}-G_{2}{ }^{j i} P_{R}\right)$

Or

$i \gamma^{\mu}\left(G_{2}{ }^{j i} P_{R}-G_{1}{ }_{j}{ }_{j} P_{L}\right)$

When the interaction Lagrangians given in eqs. (2.127) and (2.129) are converted to four-component spinor notation (see Problems 1 and 2 at the end of this section), there is an equivalent form in which $\mathscr{L}_{\text {int }}$ is written in terms of charge-conjugated Dirac four-component fields [after using eq. (2.128)]. Thus, the Feynman rules involving Dirac fermions can take two possible forms, as shown above. As previously noted, the direction of an arrow on a Dirac fermion line indicates the direction of the fermion 
charge flow (whereas the arrow on the Majorana fermion line is unconnected to charge flow). However, we are free to choose either a $\Psi$ or $\Psi^{c}$ line to represent a Dirac fermion at any place in a given Feynman graph. ${ }^{11}$ For any decay or scattering process, a suitable choice of either the $\Psi$-rule or the $\Psi^{c}$-rule at each vertex (the choice can be different at different vertices) will guarantee that the arrow directions on fermion lines flow continuously through the Feynman diagram. Then, to evaluate an invariant amplitude, one should traverse any continuous fermion line (either $\Psi$ or $\Psi^{c}$ ) by moving antiparallel to the direction of the fermion arrows.

For a given process, there may be a number of distinct choices for the arrow directions on the Majorana fermion lines, which may depend on whether one represents a given Dirac fermion by $\Psi$ or $\Psi^{c}$. However, different choices do not lead to independent Feynman diagrams. When computing an invariant amplitude, one first writes down the relevant Feynman diagrams with no arrows on any Majorana fermion line. The number of distinct graphs contributing to the process is then determined. Finally, one makes some choice for how to distribute the arrows on the Majorana fermion lines and how to label Dirac fermion lines (either as the field $\Psi$ or its charge conjugate $\Psi^{c}$ ) in a manner consistent with the Feynman rules for the vertices previously given. The end result for the invariant amplitude (apart from an overall unobservable phase) does not depend on the choices made for the direction of the fermion arrows.

Using the above procedure, the Feynman rules for the external fermion wave functions are the same for Dirac and Majorana fermions:

- $u(\overrightarrow{\boldsymbol{p}}, s)$ : incoming $\Psi\left[\right.$ or $\Psi^{c}$ ] with momentum $\overrightarrow{\boldsymbol{p}}$ parallel to the arrow direction,

- $\bar{u}(\overrightarrow{\boldsymbol{p}}, s)$ : outgoing $\Psi$ [or $\Psi^{c}$ ] with momentum $\overrightarrow{\boldsymbol{p}}$ parallel to the arrow direction,

- $v(\overrightarrow{\boldsymbol{p}}, s)$ : outgoing $\Psi$ [or $\Psi^{c}$ ] with momentum $\overrightarrow{\boldsymbol{p}}$ anti-parallel to the arrow direction,

- $\bar{v}(\overrightarrow{\boldsymbol{p}}, s)$ : incoming $\Psi$ [or $\Psi^{c}$ ] with momentum $\overrightarrow{\boldsymbol{p}}$ anti-parallel to the arrow direction.

We now consider the application of the Feynman rules presented above to some $2 \rightarrow 2$ scattering processes involving a Majorana fermion either as an external state or as an internal line.

\footnotetext{
${ }^{11}$ Since the charge of $\Psi^{c}$ is opposite in sign to the charge of $\Psi$, the corresponding arrow directions of the $\Psi$ and $\Psi^{c}$ lines must point in opposite directions.
} 
Example $2\left(\Psi\left(p_{1}\right) \Psi\left(p_{2}\right) \rightarrow \Phi\left(k_{1}\right) \Phi\left(k_{2}\right)\right.$ via $\Psi_{M}$-exchange $)$. Here, $\Phi$ is a charged scalar. The contributing Feynman graphs are:
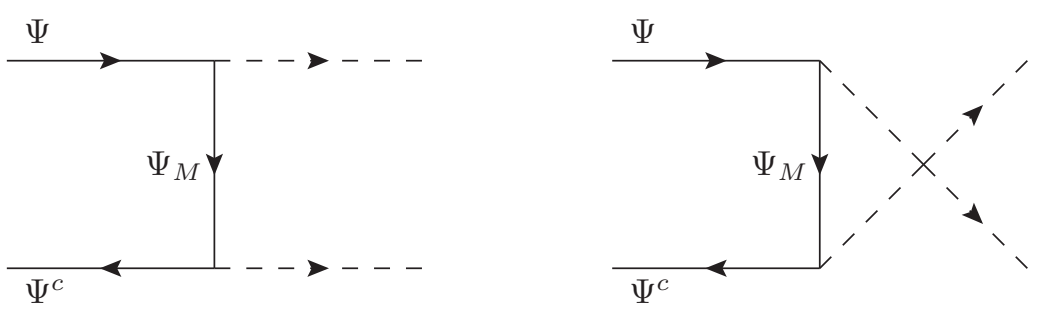

Following the arrows on the fermion lines in reverse, the invariant amplitude is given by,

$$
\begin{aligned}
i \mathcal{M}=(-i)^{2} \bar{v}\left(\overrightarrow{\boldsymbol{p}}_{2}, s_{2}\right)\left(\kappa_{1} P_{L}+\kappa_{2}^{*} P_{R}\right)\left[\frac{i\left(\not /_{1}-k_{1}+m\right)}{t-m^{2}}+\frac{i\left(k_{1}-\not p_{2}+m\right)}{u-m^{2}}\right] \\
\times\left(\kappa_{1} P_{L}+\kappa_{2}^{*} P_{R}\right) u\left(\overrightarrow{\boldsymbol{p}}_{1}, s_{1}\right),
\end{aligned}
$$

where $t \equiv\left(p_{1}-k_{1}\right)^{2}, u \equiv\left(p_{2}-k_{1}\right)^{2}$ and $m$ is the Majorana fermion mass. The sign of each diagram is determined by the relative permutation of spinor wave functions appearing in the amplitude (the overall sign of the amplitude is unphysical). In the present example, in both terms appearing in eq. (2.130), the spinor wave functions appear in the same order (first $\overrightarrow{\boldsymbol{p}}_{2}$ and then $\vec{p}_{1}$ ), implying a relative plus sign between the two terms.

One can check that $i \mathcal{M}$ is antisymmetric under interchange of the two initial electrons. This is most easily verified by taking the transpose of the invariant amplitude (the latter is a complex number whose value is not changed by transposition). It is convenient to adopt the convention in which the (commuting) $u$ and $v$ spinor wave functions are related via,

$$
\begin{array}{llrl}
v(\overrightarrow{\boldsymbol{p}}, s) & =C \bar{u}(\overrightarrow{\boldsymbol{p}}, s)^{\top}, & u(\overrightarrow{\boldsymbol{p}}, s) & =C \bar{v}(\overrightarrow{\boldsymbol{p}}, s)^{\top}, \\
\bar{v}(\overrightarrow{\boldsymbol{p}}, s) & =-u(\overrightarrow{\boldsymbol{p}}, s)^{\top} C^{-1}, & \bar{u}(\overrightarrow{\boldsymbol{p}}, s)=-v(\overrightarrow{\boldsymbol{p}}, s)^{\top} C^{-1} .
\end{array}
$$

where $C$ is the charge conjugation matrix. Using eqs. (2.131) and (2.132), the transposed amplitude can be simplified by employing the relation,

$$
\bar{v}\left(\overrightarrow{\boldsymbol{p}}_{2}, s_{2}\right) \Gamma u\left(\overrightarrow{\boldsymbol{p}}_{1}, s_{1}\right)=-\eta_{\Gamma}^{C} \bar{v}\left(\overrightarrow{\boldsymbol{p}}_{1}, s_{1}\right) \Gamma u\left(\overrightarrow{\boldsymbol{p}}_{2}, s_{2}\right),
$$

which is a consequence of eq. (2.108).

Example $3\left(\Psi\left(p_{1}\right) \Psi^{c}\left(p_{2}\right) \rightarrow \Psi_{M}\left(p_{3}\right) \Psi_{M}\left(p_{4}\right)\right.$ via charged $\Phi$-exchange). In addition to a possible $s$-channel annihilation graph, the contributing Feynman graphs can be represented by either diagram set (i) or diagram set (ii) shown below, where each set contains a $t$-channel and $u$-channel graph, respectively. 
Diagram set (i):
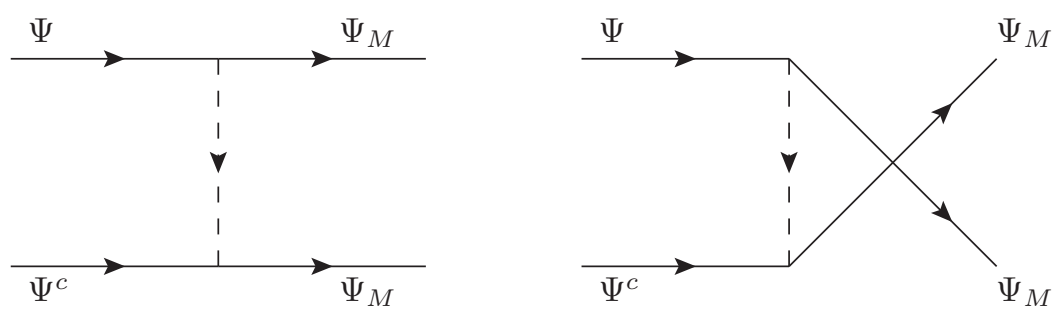

Diagram set (ii):
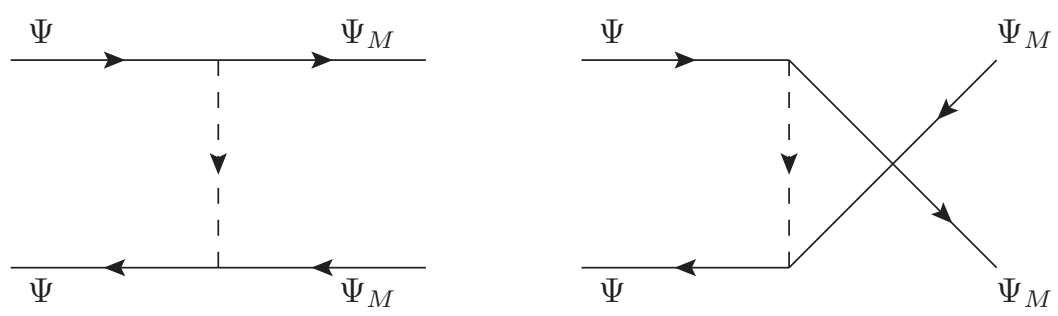

The amplitude is evaluated by following the arrows on the fermion lines in reverse. Either diagram set (i) or set (ii) may be chosen to evaluate the invariant amplitude. We again employ eq. (2.108) to derive the relation,

$$
\bar{v}\left(\overrightarrow{\boldsymbol{p}}_{2}, s_{2}\right) \Gamma v\left(\overrightarrow{\boldsymbol{p}}_{4}, s_{4}\right)=-\eta_{\Gamma}^{C} \bar{u}\left(\overrightarrow{\boldsymbol{p}}_{4}, s_{4}\right) \Gamma u\left(\overrightarrow{\boldsymbol{p}}_{2}, s_{2}\right),
$$

which can be used in comparing the invariant amplitude obtained by using diagram sets (i) and (ii). One can check that the invariant amplitudes resulting from diagram sets (i) and (ii) differ by an overall minus sign, which is unphysical. The overall minus sign arises due to the fact that the corresponding order of the spinor wave functions differs by an odd permutation [e.g., for the $t$-channel graphs, compare 3142 and 3124 for (i) and (ii) respectively]. For the same reason, there is a relative minus sign between the $t$-channel and $u$-channel graphs for either diagram set [e.g., compare 3142 and 4132 in diagram set(i)].

If $s$-channel annihilation contributes, its contribution to the invariant amplitude is easily obtained. Relative to the $t$-channel graph of diagram set (ii) above, the $s$-channel graph shown below comes with an extra minus sign (since 2134 is odd with respect to 3124 ). 


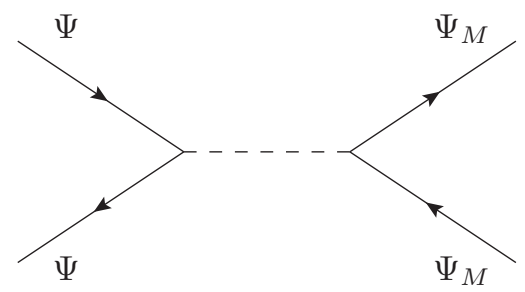

In the computation of the unpolarized cross-section, non-standard spin projection operators can arise in the evaluation of the interference terms (see Appendix D of Reference [32]), such as

$\sum_{s} u(\overrightarrow{\boldsymbol{p}}, s) v^{T}(\overrightarrow{\boldsymbol{p}}, s)=(\not p+m) C^{T}, \quad \sum_{s} \bar{u}^{T}(\overrightarrow{\boldsymbol{p}}, s) \bar{v}(\overrightarrow{\boldsymbol{p}}, s)=C^{-1}(\not p-m)$,

which requires additional manipulation of the charge conjugation matrix $C$. However, these non-standard spin projection operators can be avoided by judicious use of spinor wave function product relations of the kind obtained in eqs. (2.133) and (2.134).

\subsection{Problems}

Problem 1. Convert the interaction Lagrangian given by eq. (2.127) to four-component spinor notation. Show that the end result is

$$
\begin{aligned}
\mathscr{L}_{\text {int }}= & -\frac{1}{2}\left(\lambda^{i j} \bar{\Psi}_{M i} P_{L} \Psi_{M j}+\lambda_{i j} \bar{\Psi}_{M}^{i} P_{R} \Psi_{M}^{j}\right) \phi-\bar{\Psi}^{j}\left(\kappa_{j}^{i} P_{L}+\kappa_{i}{ }^{j} P_{R}\right) \Psi_{i} \phi \\
& -\frac{1}{2} \bar{\Psi}_{M i} \gamma^{\mu}\left[\left(G^{a}\right)_{i}{ }^{j} P_{L}-\left(G^{a}\right)_{j}{ }^{i} P_{R}\right] \Psi_{M j} \\
& -\left[\left(G_{L}^{a}\right)_{i}{ }^{j} \bar{\Psi}^{i} \gamma^{\mu} P_{L} \Psi_{j}+\left(G_{R}^{a}\right)_{i}{ }^{j} \bar{\Psi}^{i} \gamma^{\mu} P_{R} \Psi_{j}\right] A_{\mu}^{a},
\end{aligned}
$$

where the $\Psi_{M j}$ are a set of (neutral) Majorana four-component fermions and the $\Psi_{j}$ are a set of Dirac four-component fermions.

Problem 2. Convert the interaction Lagrangian given by eq. (2.129) to four-component spinor notation. Show that the end result is

$$
\begin{aligned}
\mathscr{L}_{\text {int }}= & -\left[\left(\kappa_{1}\right)^{i}{ }_{j} \bar{\Psi}^{j} P_{L} \Psi_{M i}+\left(\kappa_{2}\right)_{i j} \bar{\Psi}^{j} P_{R} \Psi_{M}^{i}\right] \Phi \\
& -\left[\left(G_{1}\right)_{j}{ }^{i} \bar{\Psi}^{j} \gamma^{\mu} P_{L} \Psi_{M i}+\left(G_{2}\right)_{i j} \bar{\Psi}^{j} \gamma^{\mu} P_{R} \Psi_{M}^{i}\right] W_{\mu}+\text { h.c. }
\end{aligned}
$$

Problem 3. Derive eq. (2.133). Then, verify that the invariant amplitude given by eq. (2.130) is antisymmetric under the interchange of the two initial electrons.

Problem 4. Derive eq. (2.134). Then, verify that the invariant amplitude for the scattering process considered in Example 3 obtained from diagram sets (i) and (ii), respectively, differ by an overall minus sign. 


\section{Motivation for TeV-scale supersymmetry}

The Standard Model (SM) of particle physics has been remarkably successful for describing the observed behavior of the fundamental particles and their interactions [64]. Indeed, there are no definitive departures from the Standard Model observed in experiments conducted at high energy collider facilities. Nevertheless, some fundamental microscopic phenomena must necessarily lie outside of the purview of the SM. These include: neutrinos with non-zero mass [65]; dark matter [66]; the suppression of CP-violation in the strong interactions (the so-called strong CP problem [67]); gauge coupling unification [68]; the baryon asymmetry of the universe [69]; inflation in the early universe [70]; dark energy [71]; and the gravitational interaction. None of these phenomena can be explained within the framework of the SM alone.

Consequently, the SM should be regarded at best as a low-energy effective field theory [72], which is valid below some high energy scale. That is, new high energy scales must exist where more fundamental physics resides. In this section, we explain why one might expect to find this new physics at the TeV scale. We discuss the principle of naturalness, and how supersymmetry provides a natural mechanism for avoiding the quadratic sensitivity of the squared-masses of elementary scalar particles to ultraviolet physics.

\subsection{Why the TeV scale?}

The classical gravitational interaction lies outside the SM. Using the fundamental constants, $\hbar, c$ and Newton's gravitational constant $G_{N}$, one can construct a quantity with the units of energy called the Planck scale,

$$
M_{\mathrm{PL}} c^{2} \equiv\left(\frac{\hbar c^{5}}{G_{N}}\right)^{1 / 2} \simeq 1.2 \times 10^{19} \mathrm{GeV} .
$$

The significance of the Planck scale can be seen as follows. At the Planck energy scale, the quantum mechanical aspects of gravity can no longer be neglected. The gravitational energy of a particle of mass $m$, evaluated at its Compton wavelength, $r_{c}=\hbar /(m c)$,

$$
\Phi \sim \frac{G_{N} m^{2}}{r_{c}}=\frac{G_{N} m^{3} c}{\hbar} \lesssim 2 m c^{2},
$$

must be below $2 m c^{2}$ to avoid particle-antiparticle pair creation by the gravitational field. Hence, up to $\mathcal{O}(1)$ constants, we conclude that $m \lesssim M_{\mathrm{PL}} \cdot{ }^{12}$

\footnotetext{
${ }^{12}$ Note that for $m=M_{\mathrm{PL}}$, the Schwarzschild radius $r_{s} \equiv 2 G_{N} m / c^{2} \simeq r_{c}$, which provides additional evidence that the quantum mechanical nature of gravity cannot be neglected at energy scales above the Planck scale.
} 
Since particle-antiparticle pair creation is an inherently quantum mechanical phenomenon, quantum gravitational effects can no longer be ignored at the Planck scale. Thus, the SM cannot be a fundamental theory of particles and interactions at energy scales of order the Planck scale and above.

There must be an energy scale $\Lambda$ at which the Standard Model breaks down. Based on the arguments given above, it follows that the upper bound on $\Lambda$ is the Planck scale. But, it is possible that $\Lambda$ lies significantly below the Planck scale. For example, a credible theory of neutrino masses (e.g., the type-I seesaw model [65]) posits the existence of a right-handed electroweak singlet Majorana neutrino of mass of order $10^{14} \mathrm{GeV}$. Henceforth, we shall define $\Lambda$ to be the lowest energy scale at which the SM breaks down.

The predictions made by the SM depend on a number of parameters that must be taken as input to the theory. These parameters cannot be predicted, since their values are sensitive to unknown ultraviolet (UV) physics. In the 1930s, it was already appreciated that a critical difference exists between the behavior of boson and fermion masses [73]. Fermion masses are logarithmically sensitive to UV physics [74] due to the chiral symmetry of massless fermions, which implies that the radiative correction to the treelevel fermion mass is of the form,

$$
\delta m_{F} \sim m_{F} \ln \left(\Lambda^{2} / m_{F}^{2}\right),
$$

which vanishes in the limit of $m_{F} \rightarrow 0$. In contrast, no such symmetry exists for bosons (in the absence of supersymmetry), and consequently we expect quadratic sensitivity of the boson squared-mass to UV physics, $\delta m_{B}^{2} \sim \Lambda^{2}$.

These observations have important consequences for the fundamental physics that describes the Higgs boson. In the SM, the Higgs boson squaredmass is given by $m_{h}^{2}=\lambda v^{2}$ and the $\mathrm{W}$ boson squared-mass is $m_{W}^{2}=\frac{1}{4} g^{2} v^{2}$, where $\left\langle\Phi^{0}\right\rangle=v / \sqrt{2}=174 \mathrm{GeV}$ is the vacuum expectation value of the neutral Higgs field, $\lambda$ is the Higgs self-coupling [cf. eq. (3.5)], and $g$ is the $\mathrm{SU}(2)$ gauge coupling. Together, these imply that

$$
\frac{m_{h}^{2}}{m_{W}^{2}}=\frac{4 \lambda}{g^{2}},
$$

which one would expect to be roughly of $\mathcal{O}(1)$. The Higgs boson with mass $125 \mathrm{GeV}$ satisfies this expectation.

However, the existence of the Higgs boson is a consequence of a spontaneously broken scalar potential,

$$
V(\Phi)=-\mu^{2}\left(\Phi^{\dagger} \Phi\right)+\frac{1}{2} \lambda\left(\Phi^{\dagger} \Phi\right)^{2},
$$

where $\mu^{2}=\frac{1}{2} \lambda v^{2}$ at the minimum of the scalar potential. The parameter $\mu^{2}$ is quadratically sensitive to $\Lambda$. Hence, to obtain $v=246 \mathrm{GeV}$ in a 
theory where $v \ll \Lambda$ requires a significant fine-tuning of the ultraviolet parameters of the fundamental theory. Indeed, the one-loop contributions to the squared mass parameter $\mu^{2}$ are expected to be of order $\left(g^{2} / 16 \pi^{2}\right) \Lambda^{2}$. Setting this quantity to be of order of $v^{2}$ (to avoid an unnatural cancellation between the tree-level parameter and the loop corrections) yields

$$
\Lambda \simeq 4 \pi v / g \sim O(1 \mathrm{TeV}) .
$$

Thus, a natural theory of electroweak symmetry breaking (EWSB) appears to require new $\mathrm{TeV}$ scale physics beyond the SM associated with the EWSB dynamics.

\subsection{The modern principle of naturalness}

This principle of naturalness was first introduced by Weisskopf in a paper published in 1939 [73]. In the abstract of this 1939 paper, Weisskopf wrote, "the self-energy of charged particles obeying Bose statistics is found to be quadratically divergent...," and concluded that in theories of elementary bosons, new phenomena must enter at an energy scale of $m / e$ (where $e$ is the relevant coupling). In modern particle physics, naturalness is often associated with the question, "how do we understand the magnitude of the EWSB scale?" In the absence of new physics beyond the SM, its natural value would be the Planck scale (or perhaps the grand unification scale or the seesaw scale that controls neutrino masses).

There have been a number of theoretical proposals to explain the origin of the EWSB energy scale: (1) naturalness is restored by a symmetry principle-supersymmetry (SUSY)-which ties the bosons to the more wellbehaved fermions [75, 76]; (2) the Higgs boson is an approximate Goldstone boson, the only other known mechanism for keeping an elementary scalar light [77]; (3) the Higgs boson is a composite scalar, with an inverse length of order the TeV-scale [77]; (4) extra spatial dimensions beyond three provide new mechanisms for naturally large hierarchies of scales [78, 79]; (5) classical scale invariance and its minimal violation via quantum anomalies [80-85] can generate a Higgs mass via dimensional transmutation [86]; and (6) the EWSB scale arises due to some vacuum selection mechanism (either anthropic [87] or cosmological [88, 89]). Finally, maybe none of these explanations are relevant, and the EWSB energy scale is simply the result of some initial condition whose origin will never be discernible.

Of course, these are lectures on supersymmetry. Thus, we shall motivate SUSY at the TeV scale as a potential solution of the so-called hierarchy problem: why is the scale of EWSB so much smaller than the Planck scale? 


\subsection{Avoiding quadratic $U V$-sensitivity with elementary scalars}

First, consider a lesson from history. The electron self-energy in classical electromagnetism goes like $e^{2} / a$, where $a$ is the classical radius of the electron. For a point-like electron, $a \rightarrow 0$; hence the electron self-energy diverges linearly. In the quantum theory, fluctuations of the electromagnetic fields (in the "single electron theory") generate a quadratic divergence. If these divergences are not canceled, one would expect QED to break down at an energy of order $m_{e} / e$, far below the Planck scale.

The linear and quadratic divergences will cancel exactly if one makes a bold hypothesis: the existence of the positron (with a mass equal to that of the electron but of opposite charge). Weisskopf was the first to demonstrate this cancellation in 1934 [74]. ${ }^{13}$ This is an historical example in which a symmetry implies the existence of a partner particle that cancels the dangerously large UV contribution to the particle mass.

The motivation for SUSY may be viewed analogously [90, 91], with the electron playing the role of SM particles and the positron playing the role of superpartners. SUSY associates a fermionic superpartner with every SM particle and vice versa, thus doubling the SM spectrum. SUSY relates the self-energy of the elementary scalar boson to the self-energy of its fermionic partner. Since the latter is only logarithmically sensitive to $\Lambda$, we conclude that the quadratic sensitivity of the scalar squared-mass to UV physics must exactly cancel. Naturalness is restored!

However, since no superpartners degenerate in mass with the corresponding SM particles exist in nature, SUSY must be a broken symmetry. Although the fundamental origin of SUSY-breaking is yet to be understood, the effective scale of SUSY-breaking cannot be much larger than of order a few TeV, if SUSY is responsible for the origin of the EWSB scale.

The absence of any evidence for SUSY at the LHC [92] is a cause for some concern [48]. This has led to some discussion of the so-called little hierarchy problem [93-95] which reflects the observation that the effective SUSY-breaking mass scale is somewhat separated from the scale of EWSB. Nevertheless, if evidence for supersymmetric phenomena in the $\mathrm{TeV}$ or multi-TeV regime were to be eventually established at the LHC or at a future collider facility (with an energy reach beyond the LHC [96]), it would be viewed as a spectacularly successful explanation of the large hierarchy between the (multi-) TeV scale and Planck scale. In this case, the remaining little hierarchy would perhaps be regarded as a less pressing issue.

${ }^{13}$ Actually the cancellation was not present in the initial publication, but thanks to a letter from Wendell Furry, the correct result was published in an erratum. 


\section{Supersymmetry: first steps}

The supersymmetry algebra is a generalization of the Lie algebra of the Poincaré group of spacetime symmetries. In this section we begin by reviewing the representations of the Poincaré group. We then present the supersymmetry algebra and examine its representations. The consequences of super-Poincaré invariance, in terms of the vacuum energy and the bosonic and fermionic degrees of freedom, are discussed. Finally, we exhibit how these properties are manifested in the simplest supersymmetric field theory of spin-0 and spin- $\frac{1}{2}$ particles (the so-called Wess-Zumino model [97]), and demonstrate how the SUSY algebra is realized.

\subsection{Review of the Poincaré algebra}

The Poincaré group consists of Lorentz transformations and spacetime translations [50]. That is, under a Poincaré transformation, the spacetime coordinates transform as $x^{\prime \mu}=\Lambda^{\mu}{ }_{\nu} x^{\nu}+a^{\mu}$, where $\Lambda$ is given by eq. (2.3) and $a^{\mu}$ is a constant four-vector. Under a Lorentz transformation $\Lambda$ and a spacetime translation $a$, the field $\psi_{\alpha}$ of spin $s$ transforms as,

$$
\psi_{\alpha}^{\prime}(x)=\exp \left(-\frac{1}{2} i \theta_{\mu \nu} S^{\mu \nu}\right)_{\alpha}{ }^{\beta} \psi_{\beta}\left(\Lambda^{-1}(x-a)\right),
$$

where we have used $x=\Lambda^{-1}\left(x^{\prime}-a\right)$ and redefined the dummy variable $x^{\prime}$ by removing the prime. The Poincaré algebra is obtained by considering an infinitesimal Poincaré transformation. Expanding in a Taylor series about $\Lambda=\mathbb{1}_{4 \times 4}$ and $a=0$, we may rewrite eq. (4.1) as ${ }^{14}$

$$
\psi_{\alpha}^{\prime}(x) \simeq\left[\mathbb{1}+i a_{\mu} P^{\mu}-\frac{i}{2} \theta_{\mu \nu}\left(L^{\mu \nu}+S^{\mu \nu}\right)\right]_{\alpha}{ }^{\beta} \psi_{\beta}(x),
$$

where $\mathbb{1}$ is the unit operator, $P^{\mu} \equiv i \partial^{\mu}$ and $L^{\mu \nu} \equiv i\left(x^{\mu} \partial^{\nu}-x^{\nu} \partial^{\mu}\right)$ are the linear and angular momentum operators, respectively, and $S^{\mu \nu}$ depends on the representation; for spin- $\frac{1}{2}$ two-component fermions,

$$
S^{\mu \nu}= \begin{cases}\sigma^{\mu \nu} & \text { for }\left(\frac{1}{2}, 0\right) \text { fields; } \\ \bar{\sigma}^{\mu \nu} & \text { for }\left(0, \frac{1}{2}\right) \text { fields. }\end{cases}
$$

The Poincaré algebra consists of ten generators $P^{\mu}$ and $J^{\mu \nu} \equiv L^{\mu \nu}+S^{\mu \nu}$ (where $J^{\mu \nu}=-J^{\nu \mu}$ ), which obey the following commutation relations:

$$
\begin{aligned}
{\left[P^{\mu}, P^{\nu}\right] } & =0, \\
{\left[J^{\mu \nu}, P^{\lambda}\right] } & =i\left(g^{\nu \lambda} P^{\mu}-g^{\mu \lambda} P^{\nu}\right), \\
{\left[J^{\alpha \beta}, J^{\rho \sigma}\right] } & =i\left(g^{\beta \rho} J^{\alpha \sigma}-g^{\alpha \rho} J^{\beta \sigma}-g^{\beta \sigma} J^{\alpha \rho}+g^{\alpha \sigma} J^{\beta \rho}\right) .
\end{aligned}
$$

\footnotetext{
${ }^{14}$ The operators $\mathbb{1}, P^{\mu}$ and $L^{\mu \nu}$ include an implicit factor of $\delta_{\alpha}{ }^{\beta}$, whereas the spin operator $S^{\mu \nu}$ depends non-trivially on $\alpha$ and $\beta$ (except for the case of spin zero, when $S=0$ ).
} 
The Poincaré algebra possesses two independent Casimir operators (these are polynomial functions of the generators that commute with the generators $P^{\mu}$ and $J^{\mu \nu}$ ), which are given by

$$
P^{2} \equiv P_{\mu} P^{\mu} \quad \text { and } \quad w^{2} \equiv w_{\mu} w^{\mu}
$$

where $w^{\mu}$ is the Pauli-Lubanski vector,

$$
w^{\mu} \equiv-\frac{1}{2} \epsilon^{\mu \nu \rho \lambda} J_{\nu \rho} P_{\lambda}
$$

in a convention where $\epsilon^{0123}=1$. Explicitly,

$$
w^{\mu}=\left(\overrightarrow{\boldsymbol{J}} \cdot \overrightarrow{\boldsymbol{P}} ; P^{0} \overrightarrow{\boldsymbol{J}}+\overrightarrow{\boldsymbol{K}} \times \overrightarrow{\boldsymbol{P}}\right),
$$

where $J^{i} \equiv \frac{1}{2} \epsilon^{i j k} J_{j k}$ and $K^{i} \equiv J^{0 i}$. Note that

$$
w_{\mu} P^{\mu}=0 \quad \text { and } \quad\left[w_{\mu}, P_{\nu}\right]=0 .
$$

The unitary representations of the Poincaré algebra can be labeled by the eigenvalues of $P^{2}$ and $w^{2}$ when acting on the physical states with nonnegative energy $P^{0}$. The eigenvalue of $P^{2}$ is $m^{2}$, where $m$ is the mass of the physical state. To see the physical interpretation of $w^{2}$, we first consider the case of $m \neq 0$. In this case, it is convenient to evaluate $w^{2}$ in the particle rest frame. In this frame, $w^{\mu}=(0 ; m \overrightarrow{\boldsymbol{S}})$, where $S^{i}$ is defined in eq. (2.8). Hence, $w^{2}=-m^{2} \overrightarrow{\boldsymbol{S}}^{2}$, with eigenvalues $-m^{2} s(s+1), s=0, \frac{1}{2}, 1, \ldots$ We conclude that massive (positive energy) states can be labeled by $(m, s)$, where $m$ is the mass and $s$ is the spin of the state.

If $m=0$, the previous analysis is not valid, since we cannot evaluate $w^{2}$ in the rest frame. Nevertheless, if we take the $m \rightarrow 0$ limit, it follows from the results above that either $w^{2}=0$, or the corresponding states have infinite spin. We reject the second possibility (which does not appear to be realized in nature), in which case $w^{2}=\lim _{m \rightarrow 0}\left(-m^{2} \overrightarrow{\boldsymbol{S}}^{2}\right)=0$. Thus, we must solve the equations, $w^{2}=P^{2}=w_{\mu} P^{\mu}=0$. It is simplest to choose a frame in which $P=P^{0}(1 ; 0,0,1)$ where $P^{0}>0$. In this frame, it is easy to show that $w=w^{0}(1 ; 0,0,1)$. That is, in any Lorentz frame,

$$
w^{\mu}=h P^{\mu},
$$

where $h$ is called the helicity operator. In particular,

$$
\left[h, P^{\mu}\right]=\left[h, J^{\mu \nu}\right]=0,
$$

which means that the eigenvalues of $h$ can be used to label states of the irreducible massless representations of the Poincaré algebra. From eq. (4.11), we derive ${ }^{15}$

$$
h=\frac{w^{0}}{P^{0}}=\frac{\overrightarrow{\boldsymbol{J}} \cdot \overrightarrow{\boldsymbol{P}}}{P^{0}}=\frac{\overrightarrow{\boldsymbol{S}} \cdot \overrightarrow{\boldsymbol{P}}}{|\overrightarrow{\boldsymbol{P}}|}=\overrightarrow{\boldsymbol{S}} \cdot \hat{\boldsymbol{P}},
$$

\footnotetext{
${ }^{15}$ We define the differential operator $L^{i} \equiv \frac{1}{2} \epsilon^{i j k} L_{j k}$. Then, noting that $\overrightarrow{\boldsymbol{L}}=\overrightarrow{\boldsymbol{x}} \times \overrightarrow{\boldsymbol{P}}$, it follows that $\overrightarrow{\boldsymbol{L}} \cdot \overrightarrow{\boldsymbol{P}}=0$. Hence, $\overrightarrow{\boldsymbol{J}} \cdot \overrightarrow{\boldsymbol{P}}=(\overrightarrow{\boldsymbol{L}}+\overrightarrow{\boldsymbol{S}}) \cdot \overrightarrow{\boldsymbol{P}}=\overrightarrow{\boldsymbol{S}} \cdot \overrightarrow{\boldsymbol{P}}$.
} 
after noting that $P^{0}=|\overrightarrow{\boldsymbol{P}}|$ for massless states. Eigenvalues of $h$ are called the helicity (and are denoted by $\lambda$ ); its spectrum consists of nonnegative half-integers, $\lambda=0, \pm \frac{1}{2}, \pm 1, \ldots$ Under a CPT transformation, $\lambda \rightarrow-\lambda$. Thus, in any quantum field theory realization of massless particles, both $\pm|\lambda|$ helicity states must appear in the theory. It is common to refer to a massless (positive energy) state of helicity $\lambda$ as having spin $|\lambda|$.

\subsection{The supersymmetry (SUSY) algebra}

In the 1960s, Coleman and Mandula proved a very powerful no-go theorem that showed that in quantum field theories in $3+1$ dimensional spacetime with a mass gap, the only possible symmetry incorporating Poincaré transformations and a global internal symmetry group of transformations must be a trivial tensor product of the two groups [98]. Subsequently, Haag, Lopuszański and Sohnius proved that the only possible extension of the Poincaré algebra involves the addition of new fermionic generators that transform either as a $\left(\frac{1}{2}, 0\right)$ or $\left(0, \frac{1}{2}\right)$ under the Lorentz algebra, denoted by $Q_{\alpha}^{i}$ and its hermitian conjugate $Q_{\dot{\alpha} i}^{\dagger} \equiv\left(Q_{\alpha}^{i}\right)^{\dagger}$, respectively, where $i=1,2, \ldots N[9,99]$. In these lectures, we shall focus exclusively on the case of $N=1$, in which case the subscript $i$ can be dropped.

We therefore begin by examining the structure of the $N=1$ SUSY algebra, which is obtained by adding one $\left(\frac{1}{2}, 0\right)$ and one $\left(0, \frac{1}{2}\right)$ generator to the Poincaré algebra, denoted by $Q_{\alpha}$ and $Q_{\dot{\alpha}}^{\dagger}$, respectively. These twocomponent spinor generators have no explicit dependence on the spacetime coordinate and are thus invariant under spacetime translations. That is,

$$
\begin{aligned}
& \exp \left(-i a_{\mu} P^{\mu}\right) Q_{\alpha} \exp \left(i a_{\mu} P^{\mu}\right)=Q_{\alpha}, \\
& \exp \left(-i a_{\mu} P^{\mu}\right) Q_{\dot{\alpha}}^{\dagger} \exp \left(i a_{\mu} P^{\mu}\right)=Q_{\dot{\alpha}}^{\dagger},
\end{aligned}
$$

where the $a_{\mu}$ are real parameters. Working to first order in $a_{\mu}$, it follows that the spinor generators must commute with the translation generator $P^{\mu}$,

$$
\left[Q_{\alpha}, P^{\mu}\right]=\left[Q_{\dot{\alpha}}^{\dagger}, P^{\mu}\right]=0 .
$$

The commutation relations given in eq. (4.16) can also be deduced by employing the following algebraic argument. Using the known transformation properties of $Q_{\alpha}, Q_{\dot{\alpha}}^{\dagger}$ and $P^{\mu}$ under the Poincaré algebra, it follows that $\left[Q_{\alpha}, P^{\mu}\right]$ must consist of generators whose transformation properties are consistent with the tensor product,

$$
\left(\frac{1}{2}, 0\right) \otimes\left(\frac{1}{2}, \frac{1}{2}\right)=\left(1, \frac{1}{2}\right) \oplus\left(0, \frac{1}{2}\right),
$$


under the Poincaré algebra. But according to the Haag-LopuszanskiSohnius theorem, there are no $\left(1, \frac{1}{2}\right)$ generators. This argument still leaves open the possibility that $\left[Q_{\alpha}, P^{\mu}\right] \propto \sigma_{\alpha \dot{\beta}}^{\mu} Q^{\dagger \dot{\beta}}$. However, it can be shown using the Jacobi identity that the proportionality constant must be zero.

The transformation properties of $Q_{\alpha}$ and $Q_{\dot{\alpha}}^{\dagger}$ under the Poincaré algebra yield their commutation relations with the $J^{\mu \nu}$,

$$
\left[Q_{\alpha}, J^{\mu \nu}\right]=\left(\sigma^{\mu \nu}\right)_{\alpha}^{\beta} Q_{\beta}, \quad\left[Q_{\dot{\alpha}}^{\dagger}, J^{\mu \nu}\right]=-Q_{\dot{\beta}}^{\dagger}\left(\bar{\sigma}^{\mu \nu}\right)_{\dot{\alpha}}^{\dot{\beta}} .
$$

The Coleman-Mandula theorem implies that one cannot obtain a consistent algebraic structure by postulating commutation relations for the $Q_{\alpha}$ and $Q_{\dot{\alpha}}^{\dagger}$. However, by declaring $Q_{\alpha}$ and $Q_{\dot{\alpha}}^{\dagger}$ to be fermionic generators, one can postulate anticommutation relations for $Q_{\alpha}$ and $Q_{\dot{\alpha}}^{\dagger}$ such that the generators $\left\{P^{\mu}, J^{\mu \nu}, Q_{\alpha}, Q_{\dot{\alpha}}^{\dagger}\right\}$ form a closed algebraic system. We therefore consider the three possible anticommutation relations, along with their transformation properties with respect to the Poincaré algebra,

$$
\begin{array}{cc}
\left\{Q_{\alpha}, Q_{\beta}\right\} & \left(\frac{1}{2}, 0\right) \otimes\left(\frac{1}{2}, 0\right)=(1,0) \oplus(0,0), \\
\left\{Q_{\dot{\alpha}}^{\dagger}, Q_{\dot{\beta}}^{\dagger}\right\} & \left(0, \frac{1}{2}\right) \otimes\left(0, \frac{1}{2}\right)=(0,1) \oplus(0,0), \\
\left\{Q_{\alpha}, Q_{\dot{\beta}}^{\dagger}\right\} & \left(\frac{1}{2}, 0\right) \otimes\left(0, \frac{1}{2}\right)=\left(\frac{1}{2}, \frac{1}{2}\right) .
\end{array}
$$

Eqs. (4.19) and (4.20) imply that

$$
\begin{gathered}
\left\{Q_{\alpha}, Q^{\beta}\right\}=s\left(\sigma^{\mu \nu}\right)_{\alpha}{ }^{\beta} J_{\mu \nu}+k \delta_{\alpha}{ }^{\beta} \mathbb{1}, \\
\left\{Q^{\dagger \dot{\alpha}}, Q_{\dot{\beta}}^{\dagger}\right\}=s^{*}\left(\bar{\sigma}^{\mu \nu}\right)^{\dot{\alpha}}{ }_{\dot{\beta}} J_{\mu \nu}+k^{*} \delta^{\dot{\alpha}}{ }_{\dot{\beta}} \mathbb{1},
\end{gathered}
$$

where $s$ and $k$ are complex numbers and eq. (4.23) is the hermitian conjugate of eq. (4.22). Note that we have raised and/or lowered some of the spinor indices for convenience. Since $\left[Q_{\alpha}, P^{\lambda}\right]=\left[Q_{\dot{\alpha}}^{\dagger}, P^{\lambda}\right]=0$ and $\left[J_{\mu \nu}, P^{\lambda}\right] \neq 0$, it follows that $s=0$. If we now lower all spinor indices, eqs. (4.22) and (4.23) with $s=0$ yield

$$
\left\{Q_{\alpha}, Q_{\beta}\right\}=k \epsilon_{\beta \alpha} \mathbb{1}, \quad\left\{Q_{\dot{\alpha}}^{\dagger}, Q_{\dot{\beta}}^{\dagger}\right\}=k^{*} \epsilon^{\dot{\beta} \dot{\alpha}} \mathbb{1},
$$

and we conclude that $k=0$, since the left-hand sides of the above equations are symmetric under the interchange of spinor indices, whereas the right hand sides are antisymmetric. Hence,

$$
\left\{Q_{\alpha}, Q_{\beta}\right\}=\left\{Q_{\dot{\alpha}}^{\dagger}, Q_{\dot{\beta}}^{\dagger}\right\}=0 \text {. }
$$

Eq. (4.21) implies that the remaining anticommutation relation must be of the form

$$
\left\{Q_{\alpha}, Q_{\dot{\beta}}^{\dagger}\right\}=t \sigma_{\alpha \dot{\beta}}^{\mu} P_{\mu}
$$


where $t$ is a complex number. Multiplying eq. (4.26) by $\bar{\sigma}^{\nu \dot{\beta} \alpha}$ and using $\operatorname{Tr}\left(\sigma^{\mu} \bar{\sigma}^{\nu}\right)=2 g^{\mu \nu}$, it follows that

$$
\bar{\sigma}_{\mu}^{\dot{\beta} \alpha}\left\{Q_{\alpha}, Q_{\dot{\beta}}^{\dagger}\right\}=2 t P_{\mu} .
$$

In particular, for $\mu=0$, eq. (4.27) relates the energy $P^{0}$ to the SUSY generators:

$$
2 t P^{0}=Q_{1} Q_{1}^{\dagger}+Q_{1}^{\dagger} Q_{1}+Q_{2} Q_{2}^{\dagger}+Q_{2}^{\dagger} Q_{2} .
$$

Since $P^{0} \geq m$ for physical states of mass $m$ and the right-hand side of eq. (4.28) is positive semi-definite, it follows that $t$ must be real and positive. ${ }^{16}$ One can rescale the definition of the fermionic generators $Q$ and $Q^{\dagger}$ such that $t=2$. In this convention,

$$
\left\{Q_{\alpha}, Q_{\dot{\beta}}^{\dagger}\right\}=2 \sigma_{\alpha \dot{\beta}}^{\mu} P_{\mu} .
$$

To summarize, the $N=1$ SUSY algebra is spanned by the generators $\left\{P^{\mu}, J^{\mu \nu}, Q_{\alpha}, Q_{\dot{\alpha}}^{\dagger}\right\}$, which satisfy eqs. (4.4)-(4.6) and

$$
\begin{aligned}
{\left[Q_{\alpha}, P^{\mu}\right] } & =\left[Q_{\dot{\alpha}}^{\dagger}, P^{\mu}\right]=0, \\
{\left[Q_{\alpha}, J^{\mu \nu}\right] } & =\left(\sigma^{\mu \nu}\right)_{\alpha}^{\beta} Q_{\beta}, \\
{\left[Q_{\dot{\alpha}}^{\dagger}, J^{\mu \nu}\right] } & =-Q_{\dot{\beta}}^{\dagger}\left(\bar{\sigma}^{\mu \nu}\right)^{\dot{\beta}} \dot{\alpha}, \\
\left\{Q_{\alpha}, Q_{\beta}\right\} & =\left\{Q_{\dot{\alpha}}^{\dagger}, Q_{\dot{\beta}}^{\dagger}\right\}=0, \\
\left\{Q_{\alpha}, Q_{\dot{\beta}}^{\dagger}\right\} & =2 \sigma_{\alpha \dot{\beta}}^{\mu} P_{\mu} .
\end{aligned}
$$

Note that eqs. (4.30)-(4.34) are unchanged under the U(1) phase transformation,

$$
Q_{\alpha} \rightarrow e^{-i \chi} Q_{\alpha}, \quad Q_{\dot{\alpha}}^{\dagger} \rightarrow e^{i \chi} Q_{\dot{\alpha}}^{\dagger},
$$

whereas the generators $P^{\mu}$ and $J^{\mu \nu}$ are not transformed. One can therefore extend the $N=1$ SUSY algebra by adding a bosonic generator $R$ such that

$$
\begin{aligned}
& e^{i \chi R} Q_{\alpha} e^{-i \chi R}=e^{-i \chi} Q_{\alpha}, \\
& e^{i \chi R} Q_{\dot{\alpha}}^{\dagger} e^{-i \chi R}=e^{i \chi} Q_{\dot{\alpha}}^{\dagger} .
\end{aligned}
$$

Expanding out to first order in $\chi$, one easily derives the commutation relations,

$$
\begin{aligned}
{\left[R, Q_{\alpha}\right] } & =-Q_{\alpha}, \\
{\left[R, Q_{\dot{\alpha}}^{\dagger}\right] } & =Q_{\dot{\alpha}}^{\dagger} .
\end{aligned}
$$

${ }^{16}$ We reject the possibility of $t=0$, in which case $Q=Q^{\dagger}=0$ and the SUSY algebra reduces to the Poincaré algebra. 
We therefore say that the generator $Q_{\alpha}$ has an $R$-charge of -1 . Since $P^{\mu}$ and $J^{\mu \nu}$ are uncharged under the $\mathrm{U}(1)_{R}$ transformation, it follows that

$$
\left[R, P^{\mu}\right]=\left[R, J^{\mu \nu}\right]=0 .
$$

Thus, eqs. (4.4)-(4.6), (4.30)-(4.34) and (4.38)-(4.40) define the maximally extended $N=1$ SUSY algebra, which includes an additional continuous $\mathrm{U}(1)_{R}$ symmetry.

\subsection{Representations of the $N=1$ SUSY algebra}

In Section 4.1, we identified the two Casimir operators of the Poincaré algebra, $P^{2}$ and $w^{2}$, and noted that the representations of the Poincare algebra can be labeled by the eigenvalues of the Casimir operators acting on the physical states. We saw that the massive representations can be labeled by their mass and spin, $(m, s)$. For a fixed value of $m$, the corresponding spin- $s$ representations are $(2 s+1)$-dimensional. For massless states, we defined the helicity operator $h=\overrightarrow{\boldsymbol{S}} \cdot \hat{\boldsymbol{P}}$ [cf. eq. (4.13)], with eigenvalues $\lambda=0, \pm \frac{1}{2}, \pm 1 \ldots$. We also noted that $\lambda$ changes sign under a CPT transformation. Hence, the massless positive energy representations of the Poincaré algebra are specified by $|\lambda|$. For the case of $\lambda=0$, the corresponding representation is one-dimensional. For any non-zero choice for $\lambda$, the corresponding representation is two-dimensional and reducible, as both $\pm|\lambda|$ helicity states must appear.

The unitary representations of the $N=1$ SUSY algebra can be determined by using similar techniques $[100,101]$. First, we identify the Casimir operators, which commute with all the SUSY algebra generators, $\left\{P^{\mu}, J^{\mu \nu}, Q_{\alpha}, Q^{\dagger \dot{\alpha}}\right\}$. It is clear that $P^{2}$ is a Casimir operator, since $Q_{\alpha}$ and $Q^{\dagger \dot{\alpha}}$ commute with $P^{\mu}$. However, $w^{2}$ is not a Casimir operator of the SUSY algebra. To establish this result, it is straightforward to use the (anti-)commutation relations of the SUSY algebra to prove that:

$$
\left[w^{\mu}, Q_{\alpha}\right]=i\left(\sigma^{\mu \nu}\right)_{\alpha}^{\beta} Q_{\beta} P_{\nu}, \quad\left[w^{\mu}, Q_{\dot{\alpha}}^{\dagger}\right]=i\left(\bar{\sigma}^{\mu \nu}\right)^{\dot{\beta}}{ }_{\alpha} Q_{\dot{\beta}}^{\dagger} P_{\nu} .
$$

Using these results, it is straightforward to derive:

$$
\begin{aligned}
{\left[w^{2}, Q_{\alpha}\right] } & =2 i \sigma_{\alpha}^{\mu \nu}{ }_{\alpha}^{\beta} Q_{\beta} w_{\mu} P_{\nu}-\frac{3}{4} P^{2} Q_{\alpha}, \\
{\left[w^{2}, Q_{\dot{\alpha}}^{\dagger}\right] } & =2 i \bar{\sigma}^{\mu \nu \dot{\beta}}{ }_{\dot{\alpha}} Q_{\dot{\beta}}^{\dagger} w_{\mu} P_{\nu}-\frac{3}{4} P^{2} Q_{\dot{\alpha}}^{\dagger}
\end{aligned}
$$

Thus, $w^{2}$ does not commute with the fermionic generators of the SUSY algebra. One consequence of this result is that the representations of the 
SUSY algebra consist of supermultiplets that contain particles of equal mass but with different spins.

In order to deduce the possible spins that make up an irreducible supermultiplet, we shall identify a second Casimir operator of the $N=1$ SUSY algebra. We begin by defining the operator

$$
B^{\mu} \equiv w^{\mu}+\frac{1}{4} Q^{\dagger} \bar{\sigma}^{\mu} Q
$$

Using eqs. (4.33), (4.34) and (4.41), one can derive

$$
\left[B^{\mu}, Q_{\alpha}\right]=-\frac{1}{2} P^{\mu} Q_{\alpha}, \quad\left[B^{\mu}, Q_{\dot{\alpha}}^{\dagger}\right]=\frac{1}{2} P^{\mu} Q_{\dot{\alpha}}^{\dagger} .
$$

The four-vector operator $B^{\mu}$ possesses some of the properties of the PauliLubanski vector $w^{\mu}$. In particular,

$$
\begin{aligned}
{\left[B^{\mu}, B^{\nu}\right] } & =i \epsilon^{\mu \nu \rho \lambda} B_{\rho} P_{\lambda} ; \\
{\left[B^{\mu}, P^{\nu}\right] } & =0 ; \\
{\left[B^{\mu}, J^{\nu \lambda}\right] } & =i\left(g^{\mu \nu} B^{\lambda}-g^{\mu \lambda} B^{\nu}\right) .
\end{aligned}
$$

One may be tempted to conjecture that $B^{2} \equiv B_{\mu} B^{\mu}$ is a Casimir operator of the SUSY algebra. However, $\left[B^{2}, Q_{\alpha}\right] \neq 0$, so we must look further. The structure of eq. (4.45) suggests that we define

$$
C^{\mu \nu} \equiv B^{\mu} P^{\nu}-B^{\nu} P^{\mu} .
$$

It then follows that

$$
\left[C^{\mu \nu}, Q_{\alpha}\right]=\left[C^{\mu \nu}, Q_{\dot{\alpha}}^{\dagger}\right]=\left[C^{\mu \nu}, P^{\lambda}\right]=0,
$$

where the first two commutators vanish as a consequence of eq. (4.45) and the last commutator vanishes as a consequence of eq. (4.47). Moreover, eqs. (4.5) and (4.48) imply that $P^{\mu}$ and $B^{\mu}$ are Lorentz four-vectors, in which case $C^{\mu \nu}$ is a second-rank Lorentz tensor. Hence

$$
C^{2} \equiv C_{\mu \nu} C^{\mu \nu}=2\left[B^{2} P^{2}-(B \cdot P)^{2}\right],
$$

satisfies

$$
\left[C^{2}, P^{\mu}\right]=\left[C^{2}, J^{\mu \nu}\right]=\left[C^{2}, Q_{\alpha}\right]=\left[C^{2}, Q_{\dot{\alpha}}^{\dagger}\right]=0 .
$$

We conclude that $P^{2}$ and $C^{2}$ are the two Casimir operators of the $N=1$ SUSY algebra. Representations of the $N=1$ SUSY algebra can therefore be labeled by the eigenvalues of $P^{2}$ and $C^{2}$ when acting on the physical states. ${ }^{17}$ The eigenvalue of $P^{2}$ is $m^{2}$, where $m$ is the mass. To understand the physical meaning of $C^{2}$, we will consider massive and massless supermultiplets separately.

${ }^{17}$ As in the case of the Poincaré algebra, we restrict our considerations to states of non-negative energy $P^{0}$. 
Table 2.: States of an $N=1$ massive supermultiplet of superspin $j$. An interpretation is provided for $j=s$ and $j=s+\frac{1}{2}$ where $s$ is a non-negative integer. The bosonic and fermionic degrees of freedom (D.o.f.) of the supermultiplet coincide and is equal to $2(2 j+1)$.

\begin{tabular}{cccc}
\hline Spin & D.o.f. & Interpretation $(j=s)$ & Interpretation $\left(j=s+\frac{1}{2}\right)$ \\
\hline$j$ & $2(2 j+1)$ & complex spin- $s$ boson & "complex" spin- $\left(s+\frac{1}{2}\right)$ fermion \\
$j+\frac{1}{2}$ & $2 j+2$ & spin- $\left(s+\frac{1}{2}\right)$ fermion & real spin- $(s+1)$ boson \\
$j-\frac{1}{2}$ & $2 j$ & spin- $\left(s-\frac{1}{2}\right)$ fermion & real spin- $s$ boson \\
\hline
\end{tabular}

\subsubsection{Massive $N=1$ supermultiplets}

To see the physical interpretation of $C^{2}$, we first consider the case of $m \neq 0$, so that we are free to evaluate the Lorentz scalar $C^{2}$ in the particle rest frame. In this frame,

$$
B^{\mu}=\left(\frac{1}{4} Q^{\dagger} \bar{\sigma}^{0} Q ; m S^{i}+\frac{1}{4} Q^{\dagger} \bar{\sigma}^{i} Q\right),
$$

where $S^{i}$ is defined in eq. (2.8). We then compute,

$$
C^{2}=2\left[B^{2} P^{2}-(B \cdot P)^{2}\right]=2 m^{2}\left[B^{2}-B_{0}^{2}\right]=-2 m^{2} B^{i} B^{i},
$$

where $B^{i} B^{i} \equiv|\overrightarrow{\boldsymbol{B}}|^{2}$. Moreover, if we define the rest-frame operator,

$$
\mathcal{J}^{i} \equiv \frac{1}{m} B^{i}=S^{i}+\frac{1}{4 m} Q^{\dagger} \bar{\sigma}^{i} Q
$$

then it follows from eq. (4.46) that

$$
\left[\mathcal{J}^{i}, \mathcal{J}^{j}\right]=i \epsilon^{i j k} \mathcal{J}^{k}
$$

The eigenvalues of $\mathcal{J}^{i} \mathcal{J}^{i}$ are $j(j+1)$ for $j=0, \frac{1}{2}, 1, \frac{3}{2} \ldots$ Hence, the eigenvalues of

$$
C^{2}=-2 m^{4} \mathcal{J}^{i} \mathcal{J}^{i}
$$

are $-2 m^{4} j(j+1)$. We conclude that for positive energy, timelike $P^{\mu}$, the unitary irreducible representations of the $N=1$ SUSY algebra are labeled by $(m, j)$, where $j$ is called the superspin of the supermultiplet. The states of an irreducible $N=1$ massive supermultiplet of superspin $j$ are exhibited in Table 2. The explicit construction of these states and a discussion of their properties is presented in Section 4.9.

Example 4 (The massive chiral supermultiplet, $j=0$ ). For $j=0$, only $j_{3}=0$ is possible, in which case the massive supermultiplet is made up of two states of spin 0 and two states of spin $\frac{1}{2}$. The two spin- 0 states can be combined into a single complex scalar state, and the two spin- $\frac{1}{2}$ states can be identified as the two components of a two-component Majorana fermion. In this case the $j-\frac{1}{2}$ row of Table 2 is not relevant. 
It can be shown (see Problem 5) that the massive supermultiplet of superspin $\frac{1}{2}$ consists of a (real) spin- 1 boson, a (real) spin- 0 boson and two mass-degenerate Majorana fermions, which can be combined into a single Dirac fermion (called a complex fermion in Table 2). As expected, in both the $j=0$ and $j=\frac{1}{2}$ cases exhibited above, the number of bosonic degrees of freedom of the supermultiplet equals the number of fermionic degrees of freedom.

\subsubsection{Massless $N=1$ supermultiplets}

We now examine the case of zero-mass positive energy states, where $P^{2}=0$ and $P^{0}>0$. If one multiplies eq. (4.34) by $P^{\rho} P^{\lambda} \bar{\sigma}_{\rho}^{\dot{\gamma} \alpha} \bar{\sigma}_{\gamma}^{\dot{\beta} \tau}$, one can easily derive the anticommutation relation,

$$
\left\{P^{\rho} \bar{\sigma}_{\rho}^{\dot{\gamma} \alpha} Q_{\alpha}, P^{\lambda} Q_{\dot{\beta}}^{\dagger} \bar{\sigma}^{\dot{\beta} \tau}\right\}=2 P^{2} P^{\mu} \bar{\sigma}_{\mu}^{\dot{\gamma} \tau} .
$$

Thus, for $P^{2}=0$ we have,

$$
\left\langle\Psi\left|\left\{P^{\rho} \bar{\sigma}_{\rho}^{\dot{\gamma} \alpha} Q_{\alpha}, P^{\lambda} Q_{\dot{\beta}}^{\dagger} \bar{\sigma}^{\dot{\beta} \tau}\right\}\right| \Psi\right\rangle=0,
$$

for any state $|\Psi\rangle$. In the space of one-particle states, only positively-normed states exist. Noting that $\left(P^{\mu} \bar{\sigma}_{\mu}^{\dot{\alpha} \beta} Q_{\beta}\right)^{\dagger}=P^{\mu} Q_{\dot{\beta}}^{\dagger} \bar{\sigma}_{\mu}^{\dot{\beta} \alpha}$, eq. (4.59) implies that as operators on the space of one-particle states,

$$
P^{\rho} \bar{\sigma}_{\rho}^{\dot{\gamma} \alpha} Q_{\alpha}=P^{\lambda} Q_{\dot{\beta}}^{\dagger} \bar{\sigma}_{\lambda}^{\dot{\beta} \tau}=0, \quad \text { for } P^{2}=0 .
$$

Using this result, one can evaluate the Casimir operator $C^{2}$, defined in eq. (4.51), in the case of $P^{2}=0$. In particular, using $w_{\mu} P^{\mu}=0$ and eq. (4.60),

$$
C^{2}=-2(B \cdot P)^{2}=-\frac{1}{8}\left(Q_{\dot{\alpha}}^{\dagger} \bar{\sigma}_{\mu}^{\dot{\alpha} \beta} Q_{\beta} P^{\mu}\right)^{2}=0 .
$$

The same conclusion can be obtained by choosing the standard reference frame, $P^{\mu}=P^{0}(1 ; 0,0,1)$, for lightlike four-vectors. In this reference frame, the anticommutators given in eqs. (4.33) and (4.34) simplify to

$$
\begin{aligned}
& \left\{Q_{1}, Q_{1}^{\dagger}\right\}=0, \quad\left\{Q_{2}, Q_{2}^{\dagger}\right\}=4 P_{0}, \\
& \left\{Q_{1}, Q_{1}\right\}=\left\{Q_{2}, Q_{2}\right\}=\left\{Q_{1}, Q_{2}\right\}=0, \\
& \left\{Q_{1}^{\dagger}, Q_{1}^{\dagger}\right\}=\left\{Q_{2}^{\dagger}, Q_{2}^{\dagger}\right\}=\left\{Q_{1}^{\dagger}, Q_{2}^{\dagger}\right\}=0 .
\end{aligned}
$$

Hence,

$$
C^{2}=-2(B \cdot P)^{2}=-\frac{1}{2} P_{0}^{2}\left(Q_{1}^{\dagger} Q_{1}\right)^{2}=\frac{1}{2} P_{0}^{2} Q_{1}^{\dagger} Q_{1}^{\dagger} Q_{1} Q_{1}=0 .
$$


Eq. (4.60) implies a number of other operator identities when acting on the space of one-particle states. Using eq. (4.34), one easily derives

$$
\left[Q^{\alpha} Q_{\alpha}, Q_{\dot{\beta}}^{\dagger}\right]=4 P_{\mu} \sigma_{\alpha \dot{\beta}}^{\mu} Q^{\alpha}, \quad\left[Q_{\dot{\alpha}}^{\dagger} Q^{\dagger \dot{\alpha}}, Q_{\beta}\right]=-4 P_{\mu} \sigma_{\alpha \dot{\beta}}^{\mu} Q^{\dagger \dot{\beta}} .
$$

Applying eq. (4.60) then yields

$$
\left[Q^{\alpha} Q_{\alpha}, Q_{\dot{\beta}}^{\dagger}\right]=\left[Q_{\dot{\alpha}}^{\dagger} Q^{\dagger \dot{\alpha}}, Q_{\beta}\right]=0, \quad \text { for } P^{2}=0 \text {. }
$$

Then, for any one-particle state $|\Psi\rangle$, eqs. (4.33), (4.34) and (4.67) yield

$$
\begin{aligned}
P_{\mu} \sigma_{\alpha \dot{\alpha}}^{\mu} Q^{\beta} Q_{\beta}|\Psi\rangle & =\frac{1}{2}\left\{Q_{\alpha}, Q_{\dot{\alpha}}^{\dagger}\right\} Q^{\beta} Q_{\beta}|\Psi\rangle=\frac{1}{2} Q_{\alpha} Q_{\dot{\alpha}}^{\dagger} Q^{\beta} Q_{\beta}|\Psi\rangle \\
& =\frac{1}{2} Q_{\alpha}\left[Q_{\dot{\alpha}}^{\dagger}, Q^{\beta} Q_{\beta}\right]|\Psi\rangle=0 .
\end{aligned}
$$

A similar computation of $P_{\mu} \sigma_{\alpha \dot{\alpha}}^{\mu} Q_{\dot{\beta}}^{\dagger} Q^{\dagger \dot{\beta}}$ allows us to conclude that

$$
P_{\mu} Q^{\beta} Q_{\beta}|\Psi\rangle=P_{\mu} Q_{\dot{\beta}}^{\dagger} Q^{\dagger \dot{\beta}}|\Psi\rangle=0, \quad \text { for } \quad P^{2}=0
$$

after multiplying through by $\bar{\sigma}_{\nu}^{\dot{\alpha} \alpha}$ and evaluating the resulting trace. As we are only interested in positive energy states, we conclude that as operators on the space of one-particle states,

$$
Q^{\beta} Q_{\beta}=Q_{\dot{\beta}}^{\dagger} Q^{\dagger \dot{\beta}}=0, \quad \text { for } P^{2}=0 \text { and } P^{0}>0 .
$$

In order to identify the massless supermultiplets of one-particle states, it is convenient to define

$$
L^{\mu} \equiv \frac{1}{2}\left(w^{\mu}+B^{\mu}\right)=w^{\mu}+\frac{1}{8} Q^{\dagger} \bar{\sigma}^{\mu} Q .
$$

Note $\left[Q_{\alpha}, P^{\mu}\right]=\left[Q_{\dot{\alpha}}^{\dagger}, P^{\mu}\right]=0$ and $\left[w_{\mu}, P_{\nu}\right]=0$ imply that

$$
\left[P^{\mu}, L^{\nu}\right]=0 \text {. }
$$

Using eqs. (4.33), (4.34) and (4.41), one can easily derive

$$
\left[L^{\mu}, Q_{\alpha}\right]=-\frac{1}{4}\left(\sigma^{\mu} \bar{\sigma}^{\nu}\right)_{\alpha}^{\beta} Q_{\beta} P_{\nu}, \quad\left[L^{\mu}, Q_{\dot{\alpha}}^{\dagger}\right]=\frac{1}{4}\left(\bar{\sigma}^{\nu} \sigma^{\mu}\right)^{\dot{\beta}}{ }_{\dot{\alpha}} Q_{\dot{\beta}}^{\dagger} P_{\nu} .
$$

A straightforward computation then gives:

$$
\left[L^{\mu}, L^{\nu}\right]=i \epsilon^{\mu \nu \rho \lambda}\left(L_{\rho}+\frac{1}{16} Q^{\dagger} \bar{\sigma}_{\rho} Q\right) P_{\lambda} .
$$

When $P^{2}=0$, we impose the results of eq. (4.60) to obtain

$$
P^{\mu} L_{\mu}=\left[L^{\mu}, Q_{\alpha}\right]=\left[L^{\mu}, Q_{\dot{\alpha}}^{\dagger}\right]=0, \quad \text { for } P^{2}=0 .
$$

Moreover, if we employ the identity

$$
\epsilon^{\mu \nu \rho \lambda} \bar{\sigma}_{\rho}=\frac{1}{2} i\left(\bar{\sigma}^{\nu} \sigma^{\mu} \bar{\sigma}^{\lambda}-\bar{\sigma}^{\lambda} \sigma^{\mu} \bar{\sigma}^{\nu}\right),
$$


[which is a consequence of eq. (2.52)], it then follows from eq. (4.60) that

$$
\epsilon^{\mu \nu \rho \lambda} Q^{\dagger} \bar{\sigma}_{\rho} Q P_{\lambda}=0, \quad \text { for } P^{2}=0 .
$$

Hence, in the massless case, eq. (4.74) simplifies to

$$
\left[L^{\mu}, L^{\nu}\right]=i \epsilon^{\mu \nu \rho \lambda} L_{\rho} P_{\lambda}, \quad \text { for } P^{2}=0 .
$$

Finally, we evaluate $L^{\mu} L_{\mu}$ for the positive energy massless one-particle states. As in the analysis of the Poincaré algebra, we shall assume that $w^{\mu} w_{\mu}=\lim _{m \rightarrow 0}\left(-m^{2} \overrightarrow{\boldsymbol{S}}^{2}\right)=0$. Using eq. (4.77), it follows that

$$
w^{\mu} Q^{\dagger} \bar{\sigma}_{\mu} Q=-\frac{1}{2} \epsilon^{\mu \nu \rho \lambda} J_{\nu \rho} P_{\lambda} Q^{\dagger} \bar{\sigma}_{\mu} Q=0 .
$$

In light of eq. (2.49), we obtain

$$
\begin{aligned}
\left(Q^{\dagger} \bar{\sigma}^{\mu} Q\right)\left(Q^{\dagger} \bar{\sigma}_{\mu} Q\right) & =2 \epsilon^{\dot{\alpha} \dot{\gamma}} \epsilon^{\beta \tau} Q_{\dot{\alpha}}^{\dagger} Q_{\beta} Q_{\dot{\gamma}}^{\dagger} Q_{\tau}=2 \epsilon^{\dot{\alpha} \dot{\gamma}} \epsilon^{\beta \tau} Q_{\dot{\alpha}}^{\dagger}\left[2 P_{\mu} \sigma_{\beta \dot{\gamma}}^{\mu}-Q_{\dot{\gamma}}^{\dagger} Q_{\beta}\right] Q_{\tau} \\
& =2\left(Q_{\dot{\alpha}}^{\dagger} Q^{\dagger \dot{\alpha}}\right)\left(Q^{\beta} Q_{\beta}\right)-4 P^{\mu} Q^{\dagger} \bar{\sigma}_{\mu} Q=0,
\end{aligned}
$$

after applying the operator identities given in eqs. (4.60) and (4.70). Hence,

$$
L^{\mu} L_{\mu}=0, \quad \text { for } P^{2}=0 \text { and } P^{0}>0 .
$$

When $P^{2}=0$ and $P^{0}>0$, the properties of $L^{\mu}$ [cf. eqs. (4.72), (4.75), (4.78) and (4.81)] match precisely the properties of the Pauli-Lubanski vector. Thus, we must solve the equations $L^{2}=P^{2}=L_{\mu} P^{\mu}=0$. In a reference frame in which $P^{\mu}=P^{0}(1 ; 0,0,1)$ and $P^{0}>0$, it follows that $L^{\mu}=L^{0}(1 ; 0,0,1)$. Consequently, in any Lorentz frame,

$$
L^{\mu}=\mathcal{K} P^{\mu},
$$

where $\mathcal{K} \equiv L^{0} / P^{0}$ is called the superhelicity operator. More explicitly, in a frame where $P^{\mu}=P^{0}(1 ; 0,0,1)$,

$$
\mathcal{K}=h+\frac{1}{8 P^{0}}\left(Q_{1}^{\dagger} Q_{1}+Q_{2}^{\dagger} Q_{2}\right),
$$

where $h \equiv w^{0} / P^{0}=\overrightarrow{\boldsymbol{S}} \cdot \hat{\boldsymbol{P}}$ is the usual helicity operator acting on massless one-particle states. By virtue of eqs. (4.30) and (4.75), it follows that

$$
\left[\mathcal{K}, P^{\mu}\right]=\left[\mathcal{K}, Q_{\alpha}\right]=\left[\mathcal{K}, Q_{\dot{\alpha}}^{\dagger}\right]=0 .
$$

Hence, the states of the massless supermultiplet are eigenstates of $\mathcal{K}$, with possible eigenvalues $\kappa=0, \pm \frac{1}{2}, \pm 1, \pm \frac{3}{2}, \ldots$. In contrast, $h$ does not commute with $Q_{\alpha}$ and $Q_{\dot{\alpha}}^{\dagger}$. Thus, the different states of the massless supermultiplet will have different helicities. We conclude that for positive energy, timelike $P^{\mu}$, the irreducible representations of the $N=1$ SUSY algebra are labeled by the eigenvalue $\kappa$ of the superhelicity operator, which is 
Table 3.: States of an $N=1$ massless supermultiplet of superhelicity $\kappa$ and the corresponding CPT conjugates which comprise an $N=1$ massless supermultiplet of superhelicity $-\kappa+\frac{1}{2}$. An interpretation is provided for $\kappa=s$ and $\kappa=s-\frac{1}{2}$, where $s$ is a positive integer. In the special case of $\kappa=\frac{1}{2}$, the scalar boson of the supermultiplet is complex, whereas for $\kappa=1, \frac{3}{2}, 2, \ldots$, the bosonic member of the supermultiplet is real with nonzero spin. In all cases, the number of bosonic and fermionic degrees of freedom (D.o.f.) coincide and are equal to 2.

\begin{tabular}{cccc}
\hline Helicities & D.o.f. & Interpretation $(\kappa=s)$ & Interpretation $\left(\kappa=s-\frac{1}{2}\right)$ \\
\hline$\kappa,-\kappa$ & 2 & spin- $s$ boson & spin- $\left(s-\frac{1}{2}\right)$ fermion \\
$\kappa-\frac{1}{2},-\kappa+\frac{1}{2}$ & 2 & spin- $\left(s-\frac{1}{2}\right)$ fermion & spin- $(s-1)$ boson \\
\hline
\end{tabular}

called the superhelicity of the massless supermultiplet. Moreover, an $N=1$ massless supermultiplet with superhelicity $\kappa$ consists of two massless states with helicity $\kappa$ and $\kappa-\frac{1}{2}$, respectively. ${ }^{18}$

Any quantum field theory realization of supersymmetry respects CPT symmetry. Since the helicity changes sign under a CPT transformation, it follows that any irreducible massless supermultiplet with superhelicity $\kappa$ must be accompanied by the corresponding CPT-conjugate states that make up an irreducible massless supermultiplet with superhelicity $-\kappa+\frac{1}{2}$. Hence, without loss of generality, we can restrict the possible values of the superhelicity to $\kappa=\frac{1}{2}, 1, \frac{3}{2}, \ldots$. These results are summarized in Table 3 . The explicit construction of the states of an irreducible massless supermultiplet and a discussion of their properties is presented in Section 4.9.

Example 5 (A massless chiral supermultiplet, with $\kappa=\frac{1}{2}$ ). Including the CPT-conjugates, this supermultiplet contains two states of helicity 0 , and two states of helicity $\pm \frac{1}{2}$, respectively, which yields a massless complex scalar and a massless Majorana fermion. We recognize this as the massless limit of a massive $j=0$ chiral supermultiplet.

Example 6 (a massless gauge supermultiplet, with $\kappa=1$ ).

Including the CPT-conjugates, this supermultiplet contains two states of helicity $\pm \frac{1}{2}$ and two states of helicity \pm 1 , which yields a massless Majorana fermion and a massless spin-1 particle. This is a gauge supermultiplet (e.g the photino and the photon of supersymmetric QED).

\footnotetext{
${ }^{18}$ In the literature, it is more common to define $L^{\mu}=\left(\mathcal{K}+\frac{1}{2}\right) P^{\mu}$, in which case the helicities of the massless $N=1$ supermultiplet are $\kappa+\frac{1}{2}$ and $\kappa$ (e.g., see refs. [4, 11]). In our opinion, the definition of the superhelicity operator given in eq. (4.82) is cleaner.
} 
In Problem 8, you will show that a massless supermultiplet with $\kappa=2$ and its CPT-conjugates contains a massless spin- $\frac{3}{2}$ and a massless spin 2 particle, which is realized in supergravity by the gravitino and the graviton, respectively.

\subsection{Consequences of super-Poincaré invariance}

A Poincaré invariant quantum field theory respects the Poincaré algebra generated by $\left\{P^{\mu}, J^{\mu \nu}\right\}$, which satisfy commutation relations given by eqs. (4.4)-(4.6). One of the basic postulates of Poincaré-invariant quantum field theory states that a translationally-invariant, Lorentz-invariant vacuum $|0\rangle$ exists such that [102],

$$
P^{\mu}|0\rangle=0, \quad J^{\mu \nu}|0\rangle=0 .
$$

In particular, $\left\langle 0\left|P^{\mu}\right| 0\right\rangle=0$. Indeed if $\left\langle 0\left|P^{\mu}\right| 0\right\rangle \neq 0$, then the vacuum would not be invariant under Lorentz transformations. This is easily proven by taking the vacuum expectation value of

$$
\exp \left(\frac{1}{2} i \theta_{\rho \tau} J^{\rho \tau}\right) P^{\mu} \exp \left(-\frac{1}{2} i \theta_{\rho \tau} J^{\rho \tau}\right)=\Lambda_{\nu}^{\mu} P^{\nu},
$$

where the $\theta_{\rho \tau}=-\theta_{\rho \tau}$ parameterize the $4 \times 4$ Lorentz transformation matrix $\Lambda^{\mu}{ }_{\nu}$ [cf. eqs. (2.3) and (2.4)]. Using $J^{\mu \nu}|0\rangle=0$, it follows that

$$
\left\langle 0\left|P^{\mu}\right| 0\right\rangle=\Lambda^{\mu}{ }_{\nu}\left\langle 0\left|P^{\nu}\right| 0\right\rangle,
$$

which holds for all Lorentz transformations $\Lambda$. Thus, it follows that $\left\langle 0\left|P^{\mu}\right| 0\right\rangle=0$.

A super-Poincaré invariant quantum field theory respects the SUSY algebra generated by $\left\{P^{\mu}, J^{\mu \nu}, Q_{\alpha}, Q^{\dagger \dot{\alpha}}\right\}$. The SUSY algebra generators satisfy the commutation relations of the Poincare algebra and the (anti)commutation relations given by eqs. (4.30)-(4.34). Two important consequences can be established:

1. The vanishing of the vacuum energy is a necessary and sufficient condition for the existence of a global supersymmetric vacuum.

2. In a theory governed by a supersymmetric action, for a fixed non-zero $P_{\mu}$ the number of bosonic and fermionic degrees of freedom coincide.

We address these two results in the next two subsections. 


\subsubsection{The vacuum energy of a globally supersymmetric theory}

In order to prove that the vanishing of the vacuum energy is a necessary and sufficient condition for the existence of a global supersymmetric vacuum, we consider the anticommutation relations of the fermionic generators of the SUSY algebra,

$$
\left\{Q_{\alpha}, Q_{\dot{\beta}}^{\dagger}\right\}=2 \sigma_{\alpha \dot{\beta}}^{\mu} P_{\mu} .
$$

Following the derivation of eq. (4.28),

$$
P^{0}=\frac{1}{4}\left[Q_{1} Q_{1}^{\dagger}+Q_{1}^{\dagger} Q_{1}+Q_{2} Q_{2}^{\dagger}+Q_{2}^{\dagger} Q_{2}\right]
$$

Since the right-hand side of eq. (4.28) is positive semi-definite (and neither $Q$ nor $Q^{\dagger}$ is the zero operator), it follows that

$$
\left\langle 0\left|P^{0}\right| 0\right\rangle=0 \quad \Longleftrightarrow \quad Q_{\alpha}|0\rangle=0 .
$$

In particular, $Q_{\alpha}|0\rangle=0$ implies that the vacuum is supersymmetric, in the same way that $P^{\mu}|0\rangle=J^{\mu \nu}|0\rangle=0$ imply that the vacuum is translationally-invariant and Lorentz-invariant. ${ }^{19}$

However, this proof is troubling for two separate reasons. First, suppose that the action of the theory is invariant under supersymmetric transformations, but the vacuum is not preserved by supersymmetry. In this case, $Q_{\alpha}|0\rangle \neq 0$, and we say that supersymmetry is spontaneously broken. Then, eq. (4.89) implies that $\left\langle 0\left|P^{0}\right| 0\right\rangle>0$, which contradicts eq. (4.85). Thus, it appears that the spontaneous breaking of supersymmetry is not possible without breaking Lorentz invariance. Perhaps a more fundamental objection is that the concept of the vacuum energy is usually considered to be unphysical in non-gravitational theories, as it is commonly asserted that only energy differences are physical. Thus, it seems to be a matter of convention to choose the vacuum energy such that $\left\langle 0\left|P^{0}\right| 0\right\rangle=0$.

To overcome the objections raised above, we re-examine the concept of the vacuum energy in relativistic (non-gravitational) quantum field theory. Using the Noether procedure, the conserved canonical energy-momentum tensor, $T_{\mu \nu}^{(c)}$ can be obtained, which satisfies $\partial^{\mu} T_{\mu \nu}^{(c)}=0 .{ }^{20}$ One can then

\footnotetext{
${ }^{19}$ Equivalently, $\left\langle 0\left|\left\{Q_{\alpha}, Q_{\dot{\beta}}^{\dagger}\right\}\right| 0\right\rangle=0$, by covariance with respect to the SUSY algebra, since there are no spinor quantities with one undotted and one dotted index that can appear on the right hand side of this equation. Hence, $Q_{\alpha}|0\rangle=0$, which then yields $\left\langle 0\left|P^{0}\right| 0\right\rangle=0$.

${ }^{20}$ The arguments given here do not depend on whether one employs the canonical energy momentum tensor or the improved symmetrized energy-momentum tensor.
} 
formally compute the vacuum energy density by summing over the vacuum Feynman diagrams of the theory. By Lorentz covariance [75],

$$
\left\langle 0\left|T_{\mu \nu}^{(c)}\right| 0\right\rangle=\mathcal{E} g_{\mu \nu}
$$

where $\mathcal{E}$ is typically UV divergent. Since the Hamiltonian density is identified as $\mathscr{H}=T_{00}$, it follows that $\mathcal{E}$ is the vacuum energy density. However, one is always free to define a new subtracted energy-momentum tensor,

$$
T_{\mu \nu} \equiv T_{\mu \nu}^{(c)}-\mathcal{E} g_{\mu \nu},
$$

which is a Lorentz-covariant expression. ${ }^{21}$ By construction, $\partial^{\mu} T_{\mu \nu}=0$ and

$$
\left\langle 0\left|T_{\mu \nu}\right| 0\right\rangle=0 .
$$

The energy-momentum tensor $T_{\mu \nu}$ plays a distinguished role in relativistic quantum field theory, since it can be used to construct the generators of spacetime translations,

$$
P_{\mu}=\int d^{3} x T_{\mu}^{0},
$$

that satisfy $\left\langle 0\left|P_{\mu}\right| 0\right\rangle=0$. Indeed, $P_{\mu}$ defined by eq. (4.94) is a four-vector with respect to Lorentz transformations. Likewise, one can construct a distinguished angular momentum tensor $M_{\mu \nu \lambda}$ that can be used to construct the generators of Lorentz transformations

$$
J_{\mu \nu}=\int d^{3} x M_{\mu \nu}^{0},
$$

which satisfy $\left\langle 0\left|J_{\mu \nu}\right| 0\right\rangle=0$.

However, in a supersymmetric theory, another choice of the energymomentum tensor is natural. The fermionic generators $Q_{\alpha}$ and $Q^{\dagger \dot{\alpha}}$ of the SUSY algebra are time-independent (conserved) quantities that are obtained by integrating the zeroth component of the supercurrents,

$$
Q_{\alpha}=\int d^{3} x J_{\alpha}^{0}, \quad Q^{\dagger \dot{\alpha}}=\int d^{3} x J^{\dagger \dot{\alpha} 0} .
$$

In a theory governed by a supersymmetric Lagrangian, the supercurrents $J_{\alpha}^{\mu}$ and $J^{\dagger \dot{\alpha} \mu}$ are related by supersymmetry to an energy-momentum tensor, denoted by $T_{\mu \nu}^{(\mathrm{SUSY})}$. Then, the proper interpretation of eq. (4.88) is [103]

$$
\left\{Q_{\alpha}, Q_{\dot{\beta}}^{\dagger}\right\}=2 \sigma_{\alpha \dot{\beta}}^{\mu} \int d^{3} x T_{\mu}^{(\mathrm{SUSY})}{ }^{0} .
$$

${ }^{21}$ For example, in the quantum theory of free fields, the vacuum energy is set to zero by defining the Hamiltonian density to be normal ordered. 
One can then rewrite the above anticommutation relation as:

$$
\left\{Q_{\alpha}, Q_{\dot{\beta}}^{\dagger}\right\}=2 \sigma_{\alpha \dot{\beta}}^{\mu} P_{\mu}+2 E_{0} \sigma_{\alpha \dot{\beta}}^{0},
$$

where $P_{\mu}$ is defined by eq. (4.94) and

$$
E_{0} \equiv \int d^{3} x\left\langle 0\left|T^{(\mathrm{SUSY})}{ }_{0}^{0}\right| 0\right\rangle \text {. }
$$

If $E_{0}=0$ (which corresponds to $T_{\mu \nu}^{(\mathrm{SUSY})}=T_{\mu \nu}$ ), then we recover the standard SUSY algebra, and the vacuum is supersymmetric. If $E_{0} \neq 0$, then eq. (4.98) is consistent with $\left\langle 0\left|P^{\mu}\right| 0\right\rangle=0$ (which is required by the Lorentz-invariant vacuum) and with $Q_{\alpha}|0\rangle \neq 0$. In particular, $E_{0}$ serves as an order parameter for broken supersymmetry.

Note that $E_{0} \geq 0$ since eq. (4.98) implies that:

$$
E_{0}=\frac{1}{4}\left\langle 0\left|Q_{1} Q_{1}^{\dagger}+Q_{1}^{\dagger} Q_{1}+Q_{2} Q_{2}^{\dagger}+Q_{2}^{\dagger} Q_{2}\right| 0\right\rangle \geq 0 .
$$

In supersymmetric theories, it is common to call $E_{0}$ the vacuum energy. Thus, if supersymmetry is spontaneously broken, then this definition of the vacuum energy is not compatible with usual conventions of quantum field theory in which the vacuum energy is defined to be zero.

Although the conclusions obtained above are correct, the derivation of eq. (4.98) is still somewhat formal. Indeed if the vacuum breaks supersymmetry, then the integrals in eq. (4.96) do not converge when integrated over an infinite volume (this is an infrared divergence), so strictly speaking the fermionic generators $Q_{\alpha}$ and $Q^{\dagger \dot{\alpha}}$ are undefined. ${ }^{22}$ Nevertheless, the supercurrents are conserved, as expected in a supersymmetric theory with no explicit supersymmetry breaking. In section 7.1.3, we will demonstrate that given a supersymmetric Lagrangian, if the vacuum breaks supersymmetry then a massless Goldstone fermion exists in the spectrum. The long range forces mediated by this massless particle are responsible for the non-convergence of the integrals in eq. (4.96). Equivalently, in a spontaneously-broken globally supersymmetric theory, applying $Q_{\alpha}$ to the vacuum creates a zero-momentum massless fermionic state, which is a state of infinite norm [25].

\subsubsection{Equality of bosonic and fermionic degrees of freedom in supersymmetric theories}

In a theory governed by a supersymmetric action, for a fixed non-zero $P_{\mu}$ the number of bosonic and fermionic degrees of freedom coincide. To prove

\footnotetext{
${ }^{22}$ Moreover, given a non-zero value for $\left\langle 0\left|T^{\text {(SUSY) }} 0{ }^{0}\right| 0\right\rangle$, which is a constant by translational invariance, one sees that $E_{0}$ defined in eq. (4.99) also diverges in the infinite volume limit.
} 
this result, we first observe that the application of $Q_{\alpha}$ or $Q_{\dot{\alpha}}^{\dagger}$ to a physical state changes that state by adding half a unit of spin. An explicit example of this behavior can be seen in eqs. (4.164) and (4.165). We can summarize this behavior in the following schematic equations,

$$
Q_{\alpha}|B\rangle=|F\rangle, \quad Q_{\alpha}|F\rangle=|B\rangle,
$$

and similarly for the application of $Q_{\dot{\alpha}}^{\dagger}$, where $|B\rangle$ is a bosonic state and $|F\rangle$ is a fermionic state. It is convenient to introduce an operator, denoted by $(-1)^{F}$, with the following properties:

$$
(-1)^{F}|B\rangle=|B\rangle, \quad(-1)^{F}|F\rangle=-|F\rangle .
$$

Note that

$$
\begin{aligned}
& Q_{\alpha}(-1)^{F}|F\rangle=-Q_{\alpha}|F\rangle=-|B\rangle, \\
& (-1)^{F} Q_{\alpha}|F\rangle=(-1)^{F}|B\rangle=|B\rangle,
\end{aligned}
$$

and similarly for the application of $Q_{\dot{\alpha}}^{\dagger}$. It follows that $Q_{\alpha}$ [and $\left.Q_{\dot{\alpha}}^{\dagger}\right]$ anticommute with $(-1)^{F}$,

$$
\left\{Q_{\alpha},(-1)^{F}\right\}=\left\{Q_{\dot{\alpha}}^{\dagger},(-1)^{F}\right\}=0 .
$$

Using eq. (4.105), we can evaluate the following trace over physical states,

$$
\begin{aligned}
\operatorname{Tr}\left[(-1)^{F}\left\{Q_{\alpha}, Q_{\dot{\beta}}^{\dagger}\right\}\right] & =\operatorname{Tr}\left[(-1)^{F}\left(Q_{\alpha} Q_{\dot{\beta}}^{\dagger}+Q_{\dot{\beta}}^{\dagger} Q_{\alpha}\right)\right] \\
& =\operatorname{Tr}\left[-Q_{\alpha}(-1)^{F} Q_{\dot{\beta}}^{\dagger}+(-1)^{F} Q_{\dot{\beta}}^{\dagger} Q_{\alpha}\right] \\
& =\operatorname{Tr}\left[-Q_{\dot{\beta}}^{\dagger} Q_{\alpha}(-1)^{F}+Q_{\dot{\beta}}^{\dagger} Q_{\alpha}(-1)^{F}\right] \\
& =0,
\end{aligned}
$$

after a cyclic permutation within the trace at the penultimate step. Employing eq. (4.34), we conclude that

$$
\operatorname{Tr}(-1)^{F}=0, \quad \text { for any fixed non-zero } P^{\mu} .
$$

For a fixed non-zero eigenvalue $p^{\mu}$ obtained by applying the momentum operator $P^{\mu}$ to a physical state,

$$
\operatorname{Tr}(-1)^{F}=\sum_{\{r\}}\left\langle p^{\mu},\{r\}\left|(-1)^{F}\right| p^{\mu},\{r\}\right\rangle=N_{B}\left(p^{\mu}\right)-N_{F}\left(p^{\mu}\right)=0,
$$

where $\{r\}$ indicates all other quantum numbers of the physical state. Thus, the number of bosonic $\left(N_{B}\right)$ and fermionic $\left(N_{F}\right)$ degrees of freedom coincide. 
We have already observed that eq. (4.107) is satisfied by all positive energy representations of the SUSY algebra. The proof above demonstrates that the equality of bosonic and fermionic degrees of freedom in supersymmetric theories is far more general. Indeed, the only case where this equality can break down is when $P^{\mu}=0$, corresponding to the vacuum state of the supersymmetric theory. ${ }^{23}$

\subsection{Supersymmetric theories of spin-O and spin- $\frac{1}{2}$ particles}

The simplest supermultiplet contains a complex scalar and a twocomponent (Majorana) fermion, of common mass $m$. The case of $m \neq 0$ corresponds to superspin $j=0$ and the case of $m=0$ corresponds to superhelicity $\frac{1}{2}$ and its CPT-conjugate.

\subsubsection{The Wess-Zumino Lagrangian}

A Lagrangian that respects the SUSY algebra is given by

$\mathscr{L}=\left(\partial_{\mu} A\right)^{\dagger}\left(\partial^{\mu} A\right)+i \psi^{\dagger} \bar{\sigma}^{\mu} \partial_{\mu} \psi-\left|\frac{d W}{d A}\right|^{2}-\frac{1}{2}\left[\frac{d^{2} W}{d A^{2}} \psi \psi+\left(\frac{d^{2} W}{d A^{2}}\right)^{\dagger} \psi^{\dagger} \psi^{\dagger}\right]$,

where $A$ is a complex scalar, ${ }^{24} \psi$ and $\psi^{\dagger}$ are two-component spinors, and $W=W(A)$ [called the superpotential] is a holomorphic function of $A$ (i.e., a function of $A$ and not $A^{\dagger}$ ). If $W(A)$ is (at most) a cubic polynomial in $A$, then the above Lagrangian yields a renormalizable quantum field theory called the Wess-Zumino model. For example, a simple quadratic superpotential, $W=\frac{1}{2} m A^{2}$, describes a free theory of a complex scalar and a Majorana fermion of common mass $|m|$. An interacting theory is obtained by including a cubic term in the superpotential,

$$
W=\frac{1}{2} m A^{2}+\frac{1}{3} g A^{3} .
$$

Without loss of generality, we can assume that $m$ and $g$ are non-negative (by appropriate rephasing of $A$ and $\psi$ ). Then, inserting eq. (4.110) into eq. (4.109) yields the Wess-Zumino Lagrangian,

$$
\begin{aligned}
\mathscr{L}= & \left(\partial_{\mu} A\right)^{\dagger}\left(\partial^{\mu} A\right)+i \psi^{\dagger} \bar{\sigma}^{\mu} \partial_{\mu} \psi-\frac{1}{2} m\left(\psi \psi+\psi^{\dagger} \psi^{\dagger}\right)-m^{2}\left(A^{\dagger} A\right) \\
& -g\left(A \psi \psi+A^{\dagger} \psi^{\dagger} \psi^{\dagger}\right)-m g\left(A^{\dagger} A\right)\left(A+A^{\dagger}\right)-g^{2}\left(A^{\dagger} A\right)^{2} .
\end{aligned}
$$

\footnotetext{
${ }^{23}$ For example, Witten showed that in an $\mathrm{SU}(N)$ supersymmetric Yang-Mills theory, $\operatorname{Tr}(-1)^{F}=N$ for the supersymmetric ground state [104].

${ }^{24}$ Employing $A$ for a complex scalar field rather than $\phi$ follows the notation first introduced in Ref. [2]. It should not be confused with the notation for a vector field, which will henceforth be denoted by $V$.
} 
As expected, the boson and fermion are mass-degenerate. Moreover, SUSY imposes relations among the couplings. In this model, we see that the quartic scalar coupling is the square of the Yukawa (scalar-fermion-fermion) coupling.

In order to employ four-component Feynman rules, it is convenient to convert the Wess-Zumino Lagrangian into four-component fermion form. Writing $A=(S+i P) / \sqrt{2}$, where $S$ and $P$ are hermitian fields, we obtain

$$
\begin{aligned}
\mathscr{L}= & \frac{1}{2}\left(\partial_{\mu} S\right)^{2}+\frac{1}{2}\left(\partial_{\mu} P\right)^{2}-\frac{1}{2} m^{2}\left(S^{2}+P^{2}\right)+\frac{1}{2} \bar{\Psi}_{M}\left(i \gamma^{\mu} \partial_{\mu}-m\right) \Psi_{M} \\
& -\frac{g}{\sqrt{2}}\left[S \bar{\Psi}_{M} \psi_{M}-i P \bar{\Psi}_{M} \gamma_{5} \Psi_{M}\right]-\frac{m g}{\sqrt{2}} S\left(S^{2}+P^{2}\right)-\frac{1}{4} g^{2}\left(S^{2}+P^{2}\right)^{2} .
\end{aligned}
$$

Note that this Lagrangian separately conserves C, P and T. We identify $S$ as a scalar and $P$ as a pseudoscalar.

\subsubsection{Invariance of the Wess-Zumino Lagrangian with respect to SUSY transformations}

The Wess-Zumino Lagrangian given by eq. (4.111) is invariant with respect to global supersymmetry transformations. Explicitly, these transformations depend on an infinitesimal Grassmann (anticommuting) two-component spinor parameter $\xi$ that is independent of the spacetime position $x$,

$$
\begin{aligned}
\delta_{\xi} A & =\sqrt{2} \xi \psi, \\
\delta_{\xi} \psi_{\alpha} & =-i \sqrt{2}\left(\sigma^{\mu} \xi^{\dagger}\right)_{\alpha} \partial_{\mu} A-\sqrt{2} \xi_{\alpha}\left(\frac{d W}{d A}\right)^{\dagger} .
\end{aligned}
$$

By hermitian conjugation, one also obtains

$$
\begin{aligned}
& \delta_{\xi} A^{\dagger}=\sqrt{2} \xi^{\dagger} \psi^{\dagger}, \\
& \delta_{\xi} \psi_{\dot{\alpha}}^{\dagger}=i \sqrt{2}\left(\xi \sigma^{\mu}\right)_{\dot{\alpha}} \partial_{\mu} A^{\dagger}-\sqrt{2} \xi_{\dot{\alpha}}^{\dagger}\left(\frac{d W}{d A}\right) .
\end{aligned}
$$

Applying these transformation laws to eq. (4.111), one obtains a result of the form

$$
\delta_{\xi} \mathscr{L}=\partial_{\mu} K^{\mu} .
$$

That is, the action of the Wess-Zumino Model, $S=\int d^{4} x \mathcal{L}$, is invariant under global SUSY transformations; i.e., $\delta_{\xi} S=0$.

But, how do we know that the transformation laws just introduced correspond to SUSY transformations? Recall that for ordinary spacetime translations,

$$
e^{i a \cdot P} \Phi(x) e^{-i a \cdot P}=\Phi(x+a),
$$


which in infinitesimal form is given by

$$
i\left[P^{\mu}, \Phi(x)\right]=\partial^{\mu} \Phi(x),
$$

where $\Phi=A$ or $\psi$. Equivalently, for an infinitesimal translation,

$$
\delta_{a} \Phi(x) \equiv \Phi(x+a)-\Phi(x) \simeq a^{\mu} \partial_{\mu} \Phi(x)=i a^{\mu}\left[P^{\mu}, \Phi(x)\right] .
$$

Likewise, since $Q$ and $Q^{\dagger}$ are the generators of SUSY-translations, we expect

$$
\delta_{\xi} \Phi(x)=i\left[\xi Q+\xi^{\dagger} Q^{\dagger}, \Phi(x)\right] .
$$

Consider the commutator of two SUSY-translations:

$$
\begin{aligned}
\left(\delta_{\eta} \delta_{\xi}-\delta_{\xi} \delta_{\eta}\right) \Phi(x) & =\left[i\left(\eta Q+\eta^{\dagger} Q^{\dagger}\right),\left[i\left(\xi Q+\xi^{\dagger} Q^{\dagger}\right), \Phi(x)\right]\right]-(\xi \longleftrightarrow \eta) \\
& =\left[\left[i\left(\eta Q+\eta^{\dagger} Q^{\dagger}\right), i\left(\xi Q+\xi^{\dagger} Q^{\dagger}\right)\right], \Phi(x)\right],
\end{aligned}
$$

after employing the Jacobi identity for the double commutators. Using the SUSY algebra,

$$
\left[\eta Q, \xi^{\dagger} Q^{\dagger}\right]=2\left(\eta \sigma^{\mu} \xi^{\dagger}\right) P_{\mu}
$$

Note that the anticommutator has been converted into a commutator due to the fact that $\eta$ and $\xi$ are anticommuting two-component spinors. Likewise,

$$
[\eta Q, \xi Q]=\left[\eta^{\dagger} Q^{\dagger}, \xi^{\dagger} Q^{\dagger}\right]=0 \text {. }
$$

Hence, we end up with

$$
\begin{aligned}
{\left[\delta_{\eta}, \delta_{\xi}\right] \Phi(x) } & =2\left(\xi \sigma^{\mu} \eta^{\dagger}-\eta^{\dagger} \sigma^{\mu} \xi^{\dagger}\right)\left[P_{\mu}, \Phi(x)\right] \\
& =-2 i\left(\xi \sigma^{\mu} \eta^{\dagger}-\eta^{\dagger} \sigma^{\mu} \xi^{\dagger}\right) \partial_{\mu} \Phi(x) .
\end{aligned}
$$

Likewise, a similar computation yields,

$$
\begin{gathered}
{\left[\delta_{\eta}, \delta_{\xi}\right] A(x)=-2 i\left(\xi \sigma^{\mu} \eta^{\dagger}-\eta^{\dagger} \sigma^{\mu} \xi^{\dagger}\right) \partial_{\mu} A(x),} \\
{\left[\delta_{\eta}, \delta_{\xi}\right] \psi_{\alpha}(x)=-2 i\left(\xi \sigma^{\mu} \eta^{\dagger}-\eta^{\dagger} \sigma^{\mu} \xi^{\dagger}\right) \partial_{\mu} \psi_{\alpha}+R,}
\end{gathered}
$$

where the remainder $R$ vanishes after imposing the classical field equations for $\psi_{\alpha}(x)$, as you will verify in Problem 10. We conclude that the SUSY algebra is realized on-shell, i.e., after employing the classical field equations.

It is instructive to employ Noether's theorem, which states that an invariance of the action under a continuous symmetry implies the existence 
of a conserved current. Since we have explicitly identified the SUSY transformations, we can use Noether's theorem to determine the corresponding conserved supercurrent. Using $\delta_{\xi} \mathscr{L}=\partial_{\mu} K^{\mu}$, the resulting conserved Noether supercurrents are

$$
\xi^{\alpha} J_{\alpha}^{\mu}+\xi_{\dot{\alpha}}^{\dagger} J^{\dagger \mu \dot{\alpha}}=\sum_{\Phi} \delta_{\xi} \Phi \frac{\delta \mathscr{L}}{\delta\left(\partial_{\mu} \Phi\right)}-K^{\mu},
$$

where the sum is taken over $\Phi=A, \psi$. Note that the supercurrent has both a Lorentz index and a spinor index. Noether's theorem states that the supercurrent is conserved after imposing the classical field equations. That is,

$$
\partial_{\mu} J_{\alpha}^{\mu}=\partial_{\mu} J^{\dagger \mu \dot{\alpha}}=0 .
$$

The supercharges are defined in the usual way (as previously noted):

$$
Q_{\alpha}=\int d^{3} x J_{\alpha}^{0}, \quad Q^{\dagger \dot{\alpha}}=\int d^{3} x J^{\dagger \dot{\alpha} 0} .
$$

These are expressions that depend on the fields $A$ and $\psi$. One can now employ the canonical commutation relations of the boson field $A$ and the canonical anticommutation relations of the fermion field $\psi$ to verify that

$$
\left\{Q_{\alpha}, Q_{\beta}\right\}=\left\{Q_{\dot{\alpha}}^{\dagger}, Q_{\dot{\beta}}^{\dagger}\right\}=0, \quad\left\{Q_{\alpha}, Q_{\dot{\beta}}^{\dagger}\right\}=2 \sigma_{\alpha \dot{\beta}}^{\mu} P_{\mu},
$$

where $P_{\mu}$ is the Noether charge of spacetime translations given in eq. (4.94).

\subsection{The SUSY algebra realized off-shell}

The SUSY transformation laws of the Wess-Zumino Lagrangian exhibited in eqs. (4.113) and (4.114) are not in an optimal form for two reasons. First, in the case of a cubic superpotential $W(A)$, the transformation law for $\psi_{\alpha}$ is non-linear in the fields. Second, the SUSY algebra is only realized onshell. We can address both these issues by introducing an auxiliary complex scalar field $F(x)$. Consider the alternative Lagrangian,

$$
\begin{aligned}
\mathscr{L}= & \left(\partial_{\mu} A\right)^{\dagger}\left(\partial^{\mu} A\right)+i \psi^{\dagger} \bar{\sigma}^{\mu} \partial_{\mu} \psi+F^{\dagger} F+F \frac{d W}{d A}+F^{\dagger}\left(\frac{d W}{d A}\right)^{\dagger} \\
& -\frac{1}{2}\left[\frac{d^{2} W}{d A^{2}} \psi \psi+\left(\frac{d^{2} W}{d A^{2}}\right)^{\dagger} \psi^{\dagger} \psi^{\dagger}\right] .
\end{aligned}
$$

The field $F(x)$ is auxiliary since $\mathscr{L}$ does not depend on $\partial_{\mu} F$ and $\partial_{\mu} F^{\dagger}$. That is, $F$ and $F^{\dagger}$ are non-dynamical fields. 
We can trivially solve for $F$ and $F^{\dagger}$ using the classical field equations,

$$
\begin{array}{lll}
\frac{\partial \mathscr{L}}{\partial F}=0 & \Longrightarrow & F^{\dagger}=-\frac{d W}{d A}, \\
\frac{\partial \mathscr{L}}{\partial F^{\dagger}}=0 & \Longrightarrow & F=-\left(\frac{d W}{d A}\right)^{\dagger} .
\end{array}
$$

Hence, eqs. (4.131) and (4.132) yield,

$$
F^{\dagger} F+F \frac{d W}{d A}+F^{\dagger}\left(\frac{d W}{d A}\right)^{\dagger}=-\left|\frac{d W}{d A}\right|^{2} .
$$

Plugging this result back into eq. (4.130), we recover the general form of the Wess-Zumino Lagrangian given by eq. (4.109).

The Lagrangian including the auxiliary fields given by eq. (4.130) is also invariant under SUSY translations. The appropriately modified SUSY transformation laws are now given by

$$
\begin{aligned}
\delta_{\xi} A & =\sqrt{2} \xi \psi, \\
\delta_{\xi} \psi_{\alpha} & =-i \sqrt{2}\left(\sigma^{\mu} \xi^{\dagger}\right)_{\alpha} \partial_{\mu} A+\sqrt{2} \xi_{\alpha} F, \\
\delta_{\xi} F & =-i \sqrt{2} \xi^{\dagger} \bar{\sigma}^{\mu} \partial_{\mu} \psi .
\end{aligned}
$$

By hermitian conjugation, one also obtains

$$
\begin{aligned}
\delta_{\xi} A^{\dagger} & =\sqrt{2} \xi^{\dagger} \psi^{\dagger}, \\
\delta_{\xi} \psi_{\dot{\alpha}}^{\dagger} & =i \sqrt{2}\left(\xi \sigma^{\mu}\right)_{\dot{\alpha}} \partial_{\mu} A^{\dagger}+\sqrt{2} \xi_{\dot{\alpha}}^{\dagger} F^{\dagger}, \\
\delta_{\xi} F^{\dagger} & =i \sqrt{2}\left(\partial_{\mu} \psi^{\dagger}\right) \bar{\sigma}^{\mu} \xi .
\end{aligned}
$$

Applying these transformation laws to eq. (4.130), one obtains a result of the form

$$
\delta_{\xi} \mathscr{L}=\partial_{\mu} K^{\prime \mu},
$$

where the explicit form for $K^{\prime \mu}$ is to be determined in Problem 13. Moreover, as you will verify in Problem 14,

$$
\left[\delta_{\eta}, \delta_{\xi}\right] \Phi(x)=-2 i\left(\xi \sigma^{\mu} \eta^{\dagger}-\eta^{\dagger} \sigma^{\mu} \xi^{\dagger}\right) \partial_{\mu} \Phi(x),
$$

for $\Phi=A, \psi$ and $F$ without the need to impose the classical field equations. Thus, the Wess-Zumino Lagrangian with auxiliary fields included as in eq. (4.130) is invariant under SUSY translations, and the SUSY algebra is realized off-shell, i.e., without requiring that the fields satisfy their classical field equations.

The following two observations will be particularly useful as we move forward. First, note that the mass dimensions of the fields are given by 
$[A]=1,[\psi]=\frac{3}{2}$ and $[F]=2$, which is consistent with the requirement that $[\mathscr{L}]=4$ (since the action is dimensionless in units of $\hbar=1)$. Then, eqs. (4.134)-(4.136) are dimensionally consistent if $[\xi]=\frac{1}{2}$. Second, note that $\delta_{\xi} F$ given in eq. (4.136) is a total derivative. Indeed, $\delta_{\xi} F$ is a total derivative as a consequence of dimensional analysis and the linearity of the SUSY transformation laws. This implies that $\delta_{\xi} F$ must involve $\partial_{\mu}$, since $\left[\partial_{\mu}\right]=1$. An important consequence of this observation is that $\int d^{4} x F$ is invariant under SUSY transformations.

\subsection{Counting bosonic and fermionic degrees of freedom}

It is instructive to count both the on-shell and off-shell bosonic and fermionic degrees of freedom in the Wess-Zumino model, which is a theory of a complex scalar and a two-component fermion.

A complex scalar possesses two real degrees of freedom. Note that applying the classical field equations (in this case the inhomogeneous KleinGordon equation) does not affect the number of scalar degrees of freedom, but only the spacetime dependence of the scalar field. The two-component fermion $\psi_{\alpha}$ possesses two complex degrees of freedom, which yields four real degrees of freedom. ${ }^{25}$ Applying the classical field equations,

$$
i \bar{\sigma}^{\mu} \partial_{\mu} \psi=\left(\frac{d^{2} W}{d A^{2}}\right)^{\dagger} \psi^{\dagger},
$$

which relate $\psi$ and $\psi^{\dagger}$, thereby eliminating two of the four degrees of freedom. ${ }^{26}$ By taking the derivative of eq. (4.142), one can eliminate $\psi^{\dagger}$ using the hermitian conjugate of eq. (4.142). The resulting equation for $\psi$ is the inhomogeneous Klein-Gordon equation, which does not further affect the number of fermionic degrees of freedom. Thus, the Wess-Zumino model possesses two on-shell bosonic and two fermionic degrees of freedom.

The counting of the off-shell degrees of freedom can be performed by examining the Lagrangian [eq. (4.130)] expressed in terms of the propagating and auxiliary fields. In this case, we count two real degrees of freedom for the complex scalar, four real degrees of freedom for the two-component fermion and two real degrees of freedom for the complex auxiliary field $F$. That is, the Wess-Zumino model possesses four bosonic and four fermionic off-shell degrees of freedom.

Thus, the number of bosonic and fermionic degrees of freedom match in both on-shell and off-shell counting.

\footnotetext{
${ }^{25}$ Equivalently, we can count $\psi$ and $\psi^{\dagger}$ as four independent degrees of freedom.

${ }^{26}$ If $d^{2} W / d A^{2}=0$, then $i \bar{\sigma}^{\mu} \partial_{\mu} \psi=0$ yields a relation between $\psi_{1}$ and $\psi_{2}$.
} 


\subsection{Lessons from the Wess-Zumino Model}

In our study of the Wess-Zumino model, we provided a Lagrangian that incorporated the fields of a known supermultiplet. However, it was rather mysterious how this Lagrangian was obtained. It was even more mysterious how we came up with the correct SUSY transformation laws for the various fields. Moreover, it was quite laborious to verify that the proposed SUSY transformation laws satisfy the SUSY algebra and the action is invariant under super-Poincaré transformations.

We also learned that in order for the SUSY transformation laws to respect the SUSY algebra off-shell, one must introduce additional auxiliary fields. One additional benefit of doing so is that the corresponding SUSY transformation laws are now linear in all the fields. For this reason, we introduced the auxiliary field $F$, which can be used to write down the SUSY translation-invariant quantity $\int d^{4} x F(x)$. This observation actually provides an important clue for how to construct a SUSY Lagrangian.

As we shall demonstrate in Section 5, it is possible to develop a formalism in which, starting with a known supermultiplet, one can trivially construct a Lagrangian that is invariant under super-Poincaré transformations. Moreover, this formalism will provide explicit forms for the SUSY transformation laws that automatically respect the SUSY algebra.

\subsection{Appendix: Constructing the states of a supermultiplet}

In this subsection, we provide further details on the construction of the states of the massive and massless supermultiplets, which yields the results presented in Tables 2 and 3 .

\subsubsection{States of a massive supermultiplet of superspin $j$}

To construct the states of the massive supermultiplet, we note that in the rest frame, the anticommutators given in eqs. (4.33) and (4.34) simplify to

$$
\begin{aligned}
& \left\{Q_{1}, Q_{1}^{\dagger}\right\}=\left\{Q_{2}, Q_{2}^{\dagger}\right\}=2 m, \\
& \left\{Q_{1}, Q_{1}\right\}=\left\{Q_{2}, Q_{2}\right\}=\left\{Q_{1}, Q_{2}\right\}=0, \\
& \left\{Q_{1}^{\dagger}, Q_{1}^{\dagger}\right\}=\left\{Q_{2}^{\dagger}, Q_{2}^{\dagger}\right\}=\left\{Q_{1}^{\dagger}, Q_{2}^{\dagger}\right\}=0 .
\end{aligned}
$$

All states in a supermultiplet with superspin $j$ are simultaneous eigenstates of $P^{2}, \mathcal{J}^{i} \mathcal{J}^{i}$ and $\mathcal{J}^{3}$ with eigenvalues $m^{2}, j(j+1)$ and $j_{3}$, respectively, where the possible values of $j_{3}$ are $-j,-j+1, \ldots, j-1, j$. 
For a fixed value of the superspin $j$, there exists a distinguished state of the supermultiplet that is a simultaneous eigenstate of $P^{2}, \mathcal{J}^{i} \mathcal{J}^{i}$ and $\mathcal{J}^{3}$, denoted by $|\Omega\rangle$, which satisfies ${ }^{27}$

$$
Q_{\beta}|\Omega\rangle=0, \quad S_{+}|\Omega\rangle=0,
$$

where $S_{ \pm} \equiv S^{1} \pm i S^{2}$. To verify that a state $|\Omega\rangle$ exists that is annihilated by $Q_{\beta}$, let us assume the contrary. Suppose that a simultaneous eigenstate of $P^{2}, \mathcal{J}^{i} \mathcal{J}^{i}$ and $\mathcal{J}^{3}$, denoted by $|\Psi\rangle$, is not annihilated by $Q_{\beta}$. In the rest frame, eq. (4.45) yields

$$
\left[\mathcal{J}^{i}, Q_{\beta}\right]=\left[\mathcal{J}^{i}, Q_{\dot{\beta}}^{\dagger}\right]=0,
$$

so it follows that $Q_{\beta}|\Psi\rangle$ is also a simultaneous eigenstate of $P^{2}, \mathcal{J}^{i} \mathcal{J}^{i}$ and $\mathcal{J}^{3}$. By assumption, $Q_{\beta}|\Psi\rangle$ is not annihilated by $Q_{\alpha}$, so we conclude that $Q_{\alpha} Q_{\beta}|\Psi\rangle$ is also a simultaneous eigenstate of $P^{2}, \mathcal{J}^{i} \mathcal{J}^{i}$ and $\mathcal{J}^{3}$. But we now arrive at a contradiction, since eq. (4.144) yields

$$
Q_{\gamma}\left(Q_{\alpha} Q_{\beta}|\Psi\rangle\right)=0
$$

Consequently, there must be at least one state of the supermultiplet that satisfies $Q_{\beta}|\Omega\rangle=0$. Using eqs. (4.55) and (4.146), it follows that

$$
\mathcal{J}^{i}|\Omega\rangle=S^{i}|\Omega\rangle \text {. }
$$

If $S_{+}|\Omega\rangle=0$, then it follows that $|\Omega\rangle$ is also a simultaneous eigenstate of $\overrightarrow{\boldsymbol{S}}^{2}$ and $S^{3}$ with corresponding eigenvalues $j(j+1)$ and $j$. Moreover, this state must be unique under the assumption that the superspin $j$ supermultiplet is an irreducible representation of the $N=1$ supersymmetry algebra.

Note that eq. (4.41) when evaluated in the rest frame yields:

$$
\left[S^{i}, Q_{\alpha}\right]=i \sigma_{\alpha}^{i 0}{ }_{\alpha}^{\beta} Q_{\beta}, \quad\left[S^{i}, Q_{\dot{\alpha}}^{\dagger}\right]=i \bar{\sigma}_{\dot{\alpha}}^{i 0 \dot{\beta}} Q_{\dot{\beta}}^{\dagger} .
$$

Hence, one can define additional states of the supermultiplet,

$$
\left|\Omega\left(j_{3}\right)\right\rangle \equiv\left(S_{-}\right)^{j-j_{3}}|\Omega\rangle, \quad \text { for } j_{3}=-j,-j+1, \ldots, j-1, j,
$$

all of which satisfy

$$
Q_{\alpha}\left|\Omega\left(j_{3}\right)\right\rangle=0
$$

${ }^{27}$ Recall that if $\left|s, m_{s}\right\rangle$ are eigenstates of $\overrightarrow{\boldsymbol{S}}^{2}$ and $S^{3}$ with corresponding eigenvalues $s(s+1)$ and $m_{s}$ respectively, then

$$
S_{ \pm}\left|s, m_{s}\right\rangle=\sqrt{\left(s \mp m_{s}\right)\left(s \pm m_{s}+1\right)}\left|s, m_{s} \pm 1\right\rangle .
$$


as a result of eq. (4.150). As before, $\mathcal{J}^{i}\left|\Omega\left(j_{3}\right)\right\rangle=S^{i}\left|\Omega\left(j_{3}\right)\right\rangle$ as a consequence of eqs. (4.55) and (4.152). It follows that $\left|\Omega\left(j_{3}\right)\right\rangle$ is also a simultaneous eigenstate of $\vec{S}^{2}$ and $S^{3}$ with corresponding eigenvalues $j(j+1)$ and $j_{3}$. That is,

$$
\left|\Omega\left(j_{3}\right)\right\rangle=\left|j, j_{3}\right\rangle
$$

where the rest-frame spin and its projection along the $z$-axis are explicitly indicated.

Starting from $\left|\Omega\left(j_{3}\right)\right\rangle=\left|j, j_{3}\right\rangle$, one can now construct the remaining states of the massive supermultiplet by considering the series of states for each possible value of $j_{3},\left|\Omega\left(j_{3}\right)\right\rangle, Q_{\dot{\alpha}}^{\dagger}\left|\Omega\left(j_{3}\right)\right\rangle, Q_{\dot{\alpha}}^{\dagger} Q_{\dot{\beta}}^{\dagger}\left|\Omega\left(j_{3}\right)\right\rangle, \ldots$ This series of states terminates due to eq. (4.144) and only four independent states survive (for a given fixed value of $j_{3}$ ),

$$
\left|\Omega\left(j_{3}\right)\right\rangle, \quad Q_{1}^{\dagger}\left|\Omega\left(j_{3}\right)\right\rangle, \quad Q_{2}^{\dagger}\left|\Omega\left(j_{3}\right)\right\rangle, \quad Q_{1}^{\dagger} Q_{2}^{\dagger}\left|\Omega\left(j_{3}\right)\right\rangle .
$$

All the states of eq. (4.154) are mass-degenerate (with mass $m \neq 0$ ). The spins of these states can be determined by applying the operators $\overrightarrow{\boldsymbol{S}}^{2}$ and $S^{3}$. By virtue of eq. (4.153), we already know that $\left|\Omega\left(j_{3}\right)\right\rangle$ is a spin- $j$ state with $S^{3}$-eigenvalue $j_{3}$. Next, one can use eq. (4.150) to derive:

$$
\begin{aligned}
{\left[S^{i}, Q_{\dot{\alpha}}^{\dagger} Q_{\dot{\beta}}^{\dagger}\right] } & =i Q_{\dot{\gamma}}^{\dagger}\left[\bar{\sigma}^{i 0 \dot{\gamma}} Q_{\dot{\alpha}}^{\dagger}-\bar{\sigma}^{i 0 \dot{\gamma}} Q_{\dot{\beta}}^{\dagger} Q_{\dot{\alpha}}^{\dagger}\right], \\
{\left[\vec{S}^{2}, Q_{\dot{\alpha}}^{\dagger} Q_{\dot{\beta}}^{\dagger}\right] } & =2 i Q_{\dot{\gamma}}^{\dagger}\left[\bar{\sigma}^{i 0 \dot{\gamma}}{ }_{\dot{\alpha}}^{\dagger} Q_{\dot{\beta}}^{\dagger}-\bar{\sigma}_{\dot{\beta}}^{i 0 \dot{\gamma}} Q_{\dot{\alpha}}^{\dagger}\right] S^{i} .
\end{aligned}
$$

It immediately follows that:

$$
\begin{gathered}
{\left[S^{i}, Q_{1}^{\dagger} Q_{2}^{\dagger}\right]=i Q_{1}^{\dagger} Q_{2}^{\dagger} \operatorname{Tr} \sigma^{i 0}=0,} \\
{\left[\overrightarrow{\boldsymbol{S}}^{2}, Q_{1}^{\dagger} Q_{2}^{\dagger}\right]=2 i Q_{1}^{\dagger} Q_{2}^{\dagger} S^{i} \operatorname{Tr} \sigma^{i 0}=0 .}
\end{gathered}
$$

Applying eqs. (4.157) and (4.158) to the state $\left|\Omega\left(j_{3}\right)\right\rangle$, it follows that $Q_{1}^{\dagger} Q_{2}^{\dagger}\left|\Omega\left(j_{3}\right)\right\rangle$ is also a spin- $j$ state with $S^{3}$-eigenvalue $j_{3}$. This result is easily understood. Noting that we can write

$$
Q_{1}^{\dagger} Q_{2}^{\dagger}=\frac{1}{2} \epsilon^{\dot{\alpha} \dot{\beta}} Q_{\dot{\alpha}}^{\dagger} Q_{\dot{\beta}}^{\dagger},
$$

it follows that $Q_{1}^{\dagger} Q_{2}^{\dagger}$ is a scalar operator. This is consistent with the fact that the antisymmetric part of the tensor product of two $\mathrm{SU}(2)$ spinor representations is an $\mathrm{SU}(2)$ singlet. Thus, $Q_{1}^{\dagger} Q_{2}^{\dagger}\left|\Omega\left(j_{3}\right)\right\rangle$ and $\left|\Omega\left(j_{3}\right)\right\rangle$ possess the same eigenvalues with respect to $\overrightarrow{\boldsymbol{S}}^{2}$ and $S^{3}$.

To determine the properties of $Q_{1}^{\dagger}\left|\Omega\left(j_{3}\right)\right\rangle$ and $Q_{2}^{\dagger}\left|\Omega\left(j_{3}\right)\right\rangle$, we first note that $Q_{\alpha}$ is a spinor operator that imparts spin- $\frac{1}{2}$ to any state it acts on. Moreover, eq. (4.150) yields:

$S^{3} Q_{1}^{\dagger}\left|\Omega\left(j_{3}\right)\right\rangle=\left(j_{3}+\frac{1}{2}\right) Q_{1}^{\dagger}\left|\Omega\left(j_{3}\right)\right\rangle, \quad S^{3} Q_{2}^{\dagger}\left|\Omega\left(j_{3}\right)\right\rangle=\left(j_{3}-\frac{1}{2}\right) Q_{2}^{\dagger}\left|\Omega\left(j_{3}\right)\right\rangle$. 
Hence, one can employ the standard results from the theory of angular momentum addition in quantum mechanics, which relates the tensor product basis to the total angular momentum basis. In particular,

$$
|j, m\rangle=\sum_{m_{1}, m_{2}}\left|j_{1}, m_{1}\right\rangle \otimes\left|j_{2}, m_{2}\right\rangle\left\langle j_{1} j_{2} ; m_{1} m_{2} \mid j m\right\rangle,
$$

where $\left\langle j_{1} j_{2} ; m_{1} m_{2} \mid j m\right\rangle$ are the Clebsch-Gordon (C-G) coefficients. We employ the Condon-Shortly phase conventions in which the C-G coefficients are real and symmetric. In the present application, we require the following two C-G coefficients (taking the upper and lower signs, respectively),

$$
\begin{aligned}
\left|\frac{1}{2}, \pm \frac{1}{2}\right\rangle \otimes\left|j, m \mp \frac{1}{2}\right\rangle= & \left(\frac{j+\frac{1}{2} \pm m}{2 j+1}\right)^{1 / 2}\left|j+\frac{1}{2}, m\right\rangle \\
& \mp\left(\frac{j+\frac{1}{2} \mp m}{2 j+1}\right)^{1 / 2}\left|j-\frac{1}{2}, m\right\rangle,
\end{aligned}
$$

Eqs. (4.153), (4.160), (4.157) and (4.158) imply that

$$
\begin{aligned}
& \left|\Omega\left(j_{3}\right)\right\rangle=\left|j, j_{3}\right\rangle, \\
& Q_{1}^{\dagger}\left|\Omega\left(j_{3}\right)\right\rangle=\left(\frac{j+j_{3}+1}{2 j+1}\right)^{1 / 2}\left|j+\frac{1}{2}, j_{3}+\frac{1}{2}\right\rangle-\left(\frac{j-j_{3}}{2 j+1}\right)^{1 / 2}\left|j-\frac{1}{2}, j_{3}+\frac{1}{2}\right\rangle, \\
& Q_{2}^{\dagger}\left|\Omega\left(j_{3}\right)\right\rangle=\left(\frac{j-j_{3}+1}{2 j+1}\right)^{1 / 2}\left|j+\frac{1}{2}, j_{3}-\frac{1}{2}\right\rangle+\left(\frac{j+j_{3}}{2 j+1}\right)^{1 / 2}\left|j-\frac{1}{2}, j_{3}-\frac{1}{2}\right\rangle,
\end{aligned}
$$

$Q_{1}^{\dagger} Q_{2}^{\dagger}\left|\Omega\left(j_{3}\right)\right\rangle=\left|j, j_{3}\right\rangle$

In particular, if $j_{3} \neq j$ then eqs. (4.164) and (4.165) imply that $Q_{1}^{\dagger}\left|\Omega\left(j_{3}\right)\right\rangle$ and $Q_{2}^{\dagger}\left|\Omega\left(j_{3}\right)\right\rangle$ are orthogonal linear combinations of spin- $\left(j \pm \frac{1}{2}\right)$ states (although these states are eigenstates of $S^{3}$ as shown in eq. (4.160)). If $j_{3}= \pm j$ then $Q_{1}^{\dagger}|\Omega(j)\rangle$ and $Q_{2}^{\dagger}|\Omega(-j)\rangle$ are states of spin- $\left(j+\frac{1}{2}\right)$, since both these states are eigenstates of $\overrightarrow{\boldsymbol{S}}^{2}$ and $S^{3}$ with eigenvalues $\left(j+\frac{1}{2}\right)\left(j+\frac{3}{2}\right)$ and $\pm\left(j+\frac{1}{2}\right)$, respectively.

Note that since $\left[P^{2}, Q_{\alpha}\right]=\left[P^{2}, Q_{\dot{\alpha}}^{\dagger}\right]=0$, it follows that all the states of the supermultiplet, $\left|\Omega\left(j_{3}\right)\right\rangle, Q^{\dagger 1}\left|\Omega\left(j_{3}\right)\right\rangle, Q^{\dagger 2}\left|\Omega\left(j_{3}\right)\right\rangle, Q^{\dagger 1} Q^{\dagger 2}\left|\Omega\left(j_{3}\right)\right\rangle$, are mass-degenerate, with common mass $m$. The states of an $N=1$ massive supermultiplet of superspin $j$ are exhibited in Table 2.

In summary, there are $4(2 j+1)$ mass-degenerate states in a massive supermultiplet of superspin $j$, which are explicitly given by eqs. (4.163)(4.166), for $j_{3}=-j,-j+1, \ldots, j-1, j$. In general, a massive supermultiplet 
of superspin $j$ is made up of $2(2 j+1)$ states of spin $j, 2 j+2$ states of spin $\left(j+\frac{1}{2}\right)$ and $2 j$ states of $\operatorname{spin}\left(j-\frac{1}{2}\right)$. The extra two states for the case of spin- $(2 j+1)$ arise when $j_{3}= \pm j$, in which cases $Q_{1}^{\dagger}|\Omega(j)\rangle$ and $Q_{2}^{\dagger}|\Omega(-j)\rangle$ are pure states of spin $\left(j+\frac{1}{2}\right)$ as previously noted. Note that the number of fermionic and bosonic degrees of freedom of the massive supermultiplet coincide and is equal to $2(2 j+1)$. These results are summarized in Table 2.

\subsubsection{States of a massless supermultiplet of superhelicity $\kappa$}

To construct the states of an irreducible massless supermultiplet, we choose the standard reference frame, $P^{\mu}=P^{0}(1 ; 0,0,1)$, for lightlike fourvectors. In this reference frame, the anticommutators given in eqs. (4.33) and (4.34) simplify to those exhibited in eqs. (4.62)-(4.64). All the states in the massless supermultiplet are simultaneous eigenstates of $P^{2}$ and the superhelicity operator $\mathcal{K}$, with eigenvalues $m^{2}$ and $\kappa$, respectively, where the possible values of $\kappa=0, \pm \frac{1}{2}, \pm 1, \pm \frac{3}{2}, \ldots$

For a fixed value of the superhelicity $\kappa$, there exists a distinct state of the supermultiplet, denoted by $|\Omega\rangle$, that satisfies:

$$
Q_{\beta}|\Omega\rangle=0, \quad \mathcal{K}|\Omega\rangle=\kappa|\Omega\rangle .
$$

To verify that a state $|\Omega\rangle$ exists that is annihilated by $Q_{\beta}$, let us assume the contrary. Suppose that a state of the massless supermultiplet, denoted by $|\Psi\rangle$ exists that is not annihilated by $Q_{\beta}$. Due to eq. (4.84), it follow that $Q_{\beta}|\Psi\rangle$ must also be a state of the massless supermultiplet. Arguing as we did below eq. (4.146), we again arrive at a contradiction. Consequently, there must be at least one state of the supermultiplet that satisfies $Q_{\beta}|\Omega\rangle=0$. Moreover, a state that satisfies eq. (4.167) must be unique under the assumption that the massless supermultiplet with superhelicity $\kappa$ is an irreducible representation of the $N=1$ SUSY algebra.

The states of the massless supermultiplet are obtained by considering the series,

$$
|\Omega\rangle, Q_{\dot{\alpha}}^{\dagger}|\Omega\rangle, Q_{\dot{\beta}}^{\dagger} Q_{\dot{\alpha}}^{\dagger}|\Omega\rangle
$$

However, $Q_{\dot{\beta}}^{\dagger} Q_{\dot{\alpha}}^{\dagger}|\Omega\rangle=0$ as a result of eq. (4.70), and $P^{\lambda} Q_{\dot{\beta}}^{\dagger} \bar{\sigma}_{\lambda}^{\dot{\beta} \tau}|\Omega\rangle=0$ as a consequence of eq. (4.60). Thus, in contrast to the massive supermultiplet, the massless supermultiplet contains only two states. These two states are eigenvalues of the helicity operator $h$. To determine the corresponding helicities, we shall employ the standard reference frame where $P^{\mu}=P^{0}(1 ; 0,0,1)$. Since eq. (4.60) yields $Q_{1}=Q_{1}^{\dagger}=0$, it follows 
that the massless $N=1$ supermultiplet consists of the two states, $|\Omega\rangle$ and $Q_{2}^{\dagger}|\Omega\rangle$. Using eqs. (4.83) and (4.167), the helicities of these two states can be determined,

$$
\begin{aligned}
h|\Omega\rangle & =\left[\mathcal{K}-\frac{1}{8 P^{0}}\left(Q_{1}^{\dagger} Q_{1}+Q_{2}^{\dagger} Q_{2}\right)\right]|\Omega\rangle=\kappa|\Omega\rangle, \\
h Q_{2}^{\dagger}|\Omega\rangle & =\left[\mathcal{K}-\frac{1}{8 P^{0}}\left(Q_{1}^{\dagger} Q_{1}+Q_{2}^{\dagger} Q_{2}\right)\right] Q_{2}^{\dagger}|\Omega\rangle \\
& =\left[\kappa Q_{2}^{\dagger}-\frac{1}{8 P^{0}} Q_{2}^{\dagger}\left(2 P_{\mu} \sigma_{22}^{\mu}-Q_{2}^{\dagger} Q_{2}\right)\right. \\
& \left.\quad-\frac{1}{8 P^{0}} Q_{1}^{\dagger}\left(2 P_{\mu} \sigma_{12}^{\mu}-Q_{2}^{\dagger} Q_{1}\right)\right]|\Omega\rangle \\
& =\left[\kappa-\frac{1}{4}\left(\sigma_{22}^{0}-\sigma_{22}^{3}\right)\right] Q_{2}^{\dagger}|\Omega\rangle=\left(\kappa-\frac{1}{2}\right) Q_{2}^{\dagger}|\Omega\rangle .
\end{aligned}
$$

Indeed, the superhelicity $\kappa$ is the maximal helicity of the massless $N=1$ supermultiplet. Thus, an irreducible $N=1$ massless supermultiplet with superhelicity $\kappa$ consists of two massless states with helicity $\kappa$ and $\kappa-\frac{1}{2}$, respectively. These results are summarized in Table 3.

\subsection{Problems}

Problem 5. Show that the massive $j=\frac{1}{2}$ supermultiplet corresponds to a real vector field, a real scalar field and a Dirac fermion field.

Problem 6. Derive the following three commutation relations:

$$
\begin{gathered}
{\left[B^{\mu}, Q_{\alpha}\right]=-\frac{1}{2} P^{\mu} Q_{\alpha}, \quad\left[B^{\mu}, Q_{\dot{\alpha}}^{\dagger}\right]=\frac{1}{2} P^{\mu} Q_{\dot{\alpha}}^{\dagger},} \\
{\left[B^{\mu}, B^{\nu}\right]=i \epsilon^{\mu \nu \rho \lambda} B_{\rho} P_{\lambda},}
\end{gathered}
$$

where $B^{\mu}$ is defined in eq. (4.53).

Problem 7. Derive the following two commutation relations,

$$
\left[L^{\mu}, Q_{\alpha}\right]=-\frac{1}{4}\left(\sigma^{\mu} \bar{\sigma}^{\nu}\right)_{\alpha}^{\beta} Q_{\beta} P_{\nu}, \quad\left[L^{\mu}, Q_{\dot{\alpha}}^{\dagger}\right]=\frac{1}{4}\left(\bar{\sigma}^{\nu} \sigma^{\mu}\right)^{\dot{\beta}}{ }_{\alpha} Q_{\dot{\beta}}^{\dagger} P_{\nu},
$$

where $L^{\mu}$ is defined in eq. (4.71).

Problem 8. Show that a massless supermultiplet with $\kappa=2$ and its CPTconjugates corresponds to a massless spin- $\frac{3}{2}$ and a massless spin 2 particle, which is realized in supergravity by the gravitino and the graviton.

Problem 9. Obtain the explicit form for $K^{\mu}$ in eq. (4.117). 
Problem 10. Obtain an explicit expression for $R(x)$ in eq. (4.125), and show that it vanishes after imposing the classical field equations for $\psi_{\alpha}(x)$. Note that this computation is non-trivial and requires a judicious application of Fierz identities for two-component fermions (which can be found, e.g., in Appendix B of Ref. [1]).

Problem 11. Obtain an explicit expression for $J_{\alpha}^{\mu}$ in terms of the fields $A$ and $\psi$ in the Wess-Zumino model.

Problem 12. Verify, for the Wess-Zumino model, that the Noether supercharges defined by eq. (4.128) satisfy the SUSY algebra [cf. eq. (4.129)].

Problem 13. Obtain the explicit form for $K^{\prime \mu}$ in eq. (4.140).

Problem 14. Starting from eqs. (4.134)-(4.136), verify that

$$
\left[\delta_{\eta}, \delta_{\xi}\right] \Phi(x)=-2 i\left(\xi \sigma^{\mu} \eta^{\dagger}-\eta^{\dagger} \sigma^{\mu} \xi^{\dagger}\right) \partial_{\mu} \Phi(x),
$$

for $\Phi=A, \psi$ and $F$ without the need to impose the classical field equations.

\section{Superspace and Superfields}

In the section we introduce superspace coordinates $\theta$ and $\theta^{\dagger}$. The concept of a supersymmetry transformation is then realized as a translation in superspace. We construct superfields [105-107], which can be expanded in powers of $\theta$ and $\theta^{\dagger}$; the corresponding expansion coefficients are the fields of a supermultiplet. By introducing the spinor covariant derivative, one is able to define the derivative of a superfield that is covariant with respect to SUSY transformations. This allows us to define an irreducible chiral superfield by imposing a derivative constraint.

Employing this formalism, we demonstrate how to construct a SUSY Lagrangian for chiral superfields, and and show that the supersymmetric action can be expressed as an integral over superspace. Finally, we discuss the improved ultraviolet behavior of SUSY and introduce the celebrated non-renormalization theorem of $N=1$ supersymmetry [108, 109].

\subsection{Superspace coordinates and translations}

In Section 4 we indicated that we expect a SUSY translation to be similar to a space-time translation, where the SUSY generators $Q, Q^{\dagger}$ replace the $P^{\mu}$ of ordinary space-time translations:

$$
\delta_{\xi} \Phi(x)=i\left[\xi Q+\xi^{\dagger} Q^{\dagger}, \Phi(x)\right],
$$

for $\Phi=A, \psi$ or $F$. But what exactly is being translated? 
In this subsection, we extend spacetime by introducing Grassmann coordinates, $\theta^{\alpha}$ and $\theta_{\dot{\alpha}}^{\dagger}$. The result is an 8-dimensional superspace with coordinates $\left(x^{\mu}, \theta^{\alpha}, \theta_{\dot{\alpha}}^{\dagger}\right)$. The Grassmann coordinates are anticommuting coordinates; i.e., they satisfy anticommutation relations,

$$
\left\{\theta^{\alpha}, \theta^{\beta}\right\}=\left\{\theta_{\dot{\alpha}}^{\dagger}, \theta_{\dot{\beta}}^{\dagger}\right\}=\left\{\theta^{\alpha}, \theta_{\dot{\beta}}^{\dagger}\right\}=0 .
$$

One can also define derivatives with respect to $\theta$ and $\theta^{\dagger}$. It is convenient to introduce the following notation,

$$
\partial_{\alpha} \equiv \frac{\partial}{\partial \theta^{\alpha}}, \quad \partial_{\dot{\alpha}}^{\dagger} \equiv \frac{\partial}{\partial \theta^{\dagger^{\dot{\alpha}}}} .
$$

The derivatives with respect to $\theta$ and $\theta^{\dagger}$ are defined in the obvious way,

$$
\partial_{\alpha} \theta^{\beta}=\delta_{\alpha}^{\beta}, \quad \partial_{\dot{\alpha}}^{\dagger} \theta^{\dagger \dot{\beta}}=\delta_{\dot{\alpha}}^{\dot{\beta}} .
$$

It then follows that

$$
\partial_{\alpha} \theta_{\beta}=\partial_{\alpha}\left(\epsilon_{\beta \gamma} \theta^{\gamma}\right)=-\epsilon_{\alpha \beta}, \quad \partial_{\dot{\alpha}}^{\dagger} \theta_{\dot{\beta}}^{\dagger}=\partial_{\dot{\alpha}}^{\dagger}\left(\epsilon_{\dot{\beta} \dot{\gamma}} \theta^{\dagger \dot{\gamma}}\right)=-\epsilon_{\dot{\alpha} \dot{\beta}} .
$$

Derivatives with respect to $\theta$ and $\theta^{\dagger}$ satisfy a modified Leibniz rule,

$$
\begin{aligned}
& \partial_{\alpha}(f g)=\left(\partial_{\alpha} f\right) g+(-1)^{\varepsilon(f)} f\left(\partial_{\alpha} g\right), \\
& \partial_{\dot{\alpha}}^{\dagger}(f g)=\left(\partial_{\dot{\alpha}}^{\dagger} f\right) g+(-1)^{\varepsilon(f)} f\left(\partial_{\dot{\alpha}}^{\dagger} g\right),
\end{aligned}
$$

where

$$
\varepsilon(f)= \begin{cases}0, & \text { if } f \text { is Grassmann even }, \\ 1, & \text { if } f \text { is Grassmann odd }\end{cases}
$$

and $f$ is Grassmann even [odd] if it is a product of an even [odd] number of anticommuting quantities. For example,

$$
\begin{gathered}
\partial_{\alpha}(\theta \theta)=\partial_{\alpha}\left(\epsilon_{\gamma \beta} \theta^{\gamma} \theta^{\beta}\right)=\epsilon_{\gamma \beta}\left(\delta_{\alpha}^{\gamma} \theta^{\beta}-\delta_{\alpha}^{\beta} \theta^{\gamma}\right)=2 \theta_{\alpha}, \\
\partial_{\dot{\alpha}}^{\dagger}\left(\theta^{\dagger} \theta^{\dagger}\right)=\partial_{\dot{\alpha}}^{\dagger}\left(\epsilon_{\dot{\beta} \dot{\gamma}} \theta^{\dagger \dot{\gamma}} \theta^{\dagger \dot{\beta}}\right)=\epsilon_{\dot{\beta} \dot{\gamma}}\left(\delta_{\dot{\alpha}}^{\dot{\gamma}} \theta^{\dagger \dot{\beta}}-\delta_{\dot{\alpha}}^{\dot{\beta}} \theta^{\dagger \dot{\gamma}}\right)=-2 \theta_{\dot{\alpha}}^{\dagger} .
\end{gathered}
$$

Likewise, one conventionally defines,

$$
\partial^{\alpha} \equiv \frac{\partial}{\partial \theta_{\alpha}}, \quad \partial^{\dagger \dot{\alpha}} \equiv \frac{\partial}{\partial \theta_{\dot{\alpha}}^{\dagger}} .
$$

However, one needs to be careful since this notation leads to an unexpected minus sign when relating the derivatives of eqs. (5.3) and (5.11),

$$
\partial^{\alpha}=-\epsilon^{\alpha \beta} \partial_{\beta}, \quad \partial^{\dagger \dot{\alpha}}=-\epsilon^{\dot{\alpha} \dot{\beta}} \partial_{\dot{\beta}}^{\dagger} .
$$

This is the one case where the rule for raising a spinor index given in eq. (2.24) does not apply. 
In order to define translations in superspace, we shall generalize the translation operator $\exp (i x \cdot P)$ to the super-translation operator,

$$
G\left(x, \theta, \theta^{\dagger}\right)=\exp \left(i x \cdot P+\theta Q+\theta^{\dagger} Q^{\dagger}\right) .
$$

We can now extend the field operator, $\Phi(x)=\exp (i x \cdot P) \Phi(0) \exp (-i x \cdot P)$ to a superfield operator,

$$
\Phi\left(x, \theta, \theta^{\dagger}\right)=G\left(x, \theta, \theta^{\dagger}\right) \Phi(0,0,0) G^{-1}\left(x, \theta, \theta^{\dagger}\right) .
$$

In this way, we can realize a supersymmetry transformation as a translation in superspace.

Using the Baker-Campbell-Hausdorff formula [110],

$$
\exp (A) \exp (B)=\exp \left(A+B+\frac{1}{2}[A, B]+\cdots\right),
$$

one can prove (see Problem 15),

$$
G\left(y, \xi, \xi^{\dagger}\right) G\left(x, \theta, \theta^{\dagger}\right)=G\left(x+y+i\left(\xi \sigma \theta^{\dagger}-\theta \sigma \xi^{\dagger}\right), \xi+\theta, \xi^{\dagger}+\theta^{\dagger}\right) .
$$

Note the appearance in eq. (5.16) of an extra non-trivial spacetime translation, $i\left(\xi \sigma \theta^{\dagger}-\theta \sigma \xi^{\dagger}\right)$. Hence, it follows that

$$
\begin{aligned}
& G\left(y, \xi, \xi^{\dagger}\right) \Phi\left(x, \theta, \theta^{\dagger}\right) G^{-1}\left(y, \xi, \xi^{\dagger}\right) \\
& \quad=\Phi\left(x+y+i\left(\xi \sigma \theta^{\dagger}-\theta \sigma \xi^{\dagger}\right), \xi+\theta, \xi^{\dagger}+\theta^{\dagger}\right) .
\end{aligned}
$$

For infinitesimal $y, \xi$ and $\xi^{\dagger}$, we can approximate

$$
G\left(y, \xi, \xi^{\dagger}\right) \simeq \mathbb{1}+i\left(y \cdot P+\xi Q+\xi^{\dagger} Q^{\dagger}\right),
$$

which allows us to rewrite the left-hand side of eq. (5.17) as

$$
\begin{aligned}
G & \left(y, \xi, \xi^{\dagger}\right) \Phi\left(x, \theta, \theta^{\dagger}\right) G^{-1}\left(y, \xi, \xi^{\dagger}\right) \\
& \simeq\left(\mathbb{1}+i\left(y \cdot P+\xi Q+\xi^{\dagger} Q^{\dagger}\right)\right) \Phi\left(x, \theta, \theta^{\dagger}\right)\left(\mathbb{1}-i\left(y \cdot P+\xi Q+\xi^{\dagger} Q^{\dagger}\right)\right) \\
& \simeq \Phi\left(x, \theta, \theta^{\dagger}\right)+i y_{\mu}\left[P^{\mu}, \Phi\right]+i[\xi Q, \Phi]+i\left[\xi^{\dagger} Q^{\dagger}, \Phi\right] .
\end{aligned}
$$

One can also Taylor expand the right-hand side of eq. (5.17), which to first order yields

$$
\begin{aligned}
\Phi\left(x+y+i\left(\xi \sigma \theta^{\dagger}-\theta \sigma \xi^{\dagger}\right), \xi+\theta, \xi^{\dagger}+\theta^{\dagger}\right) \\
=\Phi\left(x, \theta, \theta^{\dagger}\right)+\left[y^{\mu}+i\left(\xi \sigma^{\mu} \theta^{\dagger}-\theta \sigma^{\mu} \xi^{\dagger}\right)\right] \partial_{\mu} \Phi\left(x, \theta, \theta^{\dagger}\right) \\
+\left(\xi^{\alpha} \partial_{\alpha}+\xi^{\dagger} \partial^{\dagger \dot{\alpha}}\right) \Phi\left(x, \theta, \theta^{\dagger}\right)
\end{aligned}
$$

where we have employed the derivatives defined in eq. (5.3). Comparing the first-order terms of eqns. (5.19) and (5.20), we end up with expressions for the following commutators,

$$
\begin{aligned}
{\left[\Phi, P_{\mu}\right] } & =i \partial_{\mu} \Phi \\
{[\Phi, \xi Q] } & =i \xi^{\alpha}\left(\partial_{\alpha}+i\left(\sigma^{\mu} \theta^{\dagger}\right)_{\alpha} \partial_{\mu}\right) \Phi, \\
{\left[\Phi, \xi^{\dagger} Q^{\dagger}\right] } & =-i\left(\partial_{\dot{\alpha}}^{\dagger}+i\left(\theta \sigma^{\mu}\right)_{\dot{\alpha}} \partial_{\mu}\right) \xi^{\dagger} \dot{\alpha} \Phi
\end{aligned}
$$


The above results motivate the introduction of the following differential operators,

$$
\begin{aligned}
& \widehat{P}_{\mu}=i \partial_{\mu}, \\
& \widehat{Q}_{\alpha}=i \partial_{\alpha}-\left(\sigma^{\mu} \theta^{\dagger}\right)_{\alpha} \partial_{\mu}, \\
& \widehat{Q}_{\dot{\alpha}}^{\dagger}=-i \partial_{\dot{\alpha}}^{\dagger}+\left(\theta \sigma^{\mu}\right)_{\dot{\alpha}} \partial_{\mu},
\end{aligned}
$$

which allow us to succinctly rewrite eqs. (5.21)-(5.23) as follows:

$$
\begin{aligned}
{\left[\Phi, P_{\mu}\right] } & =\widehat{P}_{\mu} \Phi, \\
{[\Phi, \xi Q] } & =(\xi \widehat{Q}) \Phi, \\
{\left[\Phi, \xi^{\dagger} Q^{\dagger}\right] } & =\left(\xi^{\dagger} \widehat{Q}^{\dagger}\right) \Phi .
\end{aligned}
$$

In eq. (4.121), we noted that the action of an infinitesimal SUSY transformation on any field $\Phi(x)$ was given by $\delta_{\xi} \Phi(x)=i\left[\xi Q+\xi^{\dagger} Q^{\dagger}, \Phi(x)\right]$. In light of eqs. (5.28) and (5.29), we conclude that the action of an infinitesimal SUSY transformation on a superfield $\Phi\left(x, \theta, \theta^{\dagger}\right)$ is given by

$$
\delta_{\xi} \Phi\left(x, \theta, \theta^{\dagger}\right)=-i\left(\xi \widehat{Q}+\xi^{\dagger} \widehat{Q}^{\dagger}\right) \Phi\left(x, \theta, \theta^{\dagger}\right) .
$$

\subsection{Expansion of the superfield in powers of $\theta$ and $\theta^{\dagger}$}

Consider the Taylor expansion of a superfield, $\Phi\left(x, \theta, \theta^{\dagger}\right)$, in powers of $\theta$ and $\theta^{\dagger}$. The coefficients of this expansion will be functions of $x$, which can be interpreted as ordinary fields. Since $\theta$ and $\theta^{\dagger}$ are anticommuting coordinates, this Taylor series terminates after a finite number of terms. In particular, since $\theta$ and $\theta^{\dagger}$ are anticommuting two-component spinor quantities, it follows that $\left(\theta_{1}\right)^{2}=\left(\theta_{2}\right)^{2}=\left(\theta_{\dot{1}}^{\dagger}\right)^{2}=\left(\theta_{\dot{2}}^{\dagger}\right)^{2}=0$, whereas products such as $\theta_{1} \theta_{2}$ and $\theta_{1}^{\dagger} \theta_{2}^{\dagger}$ do not vanish. Indeed, it is easy to check that

$$
\begin{array}{ll}
\theta^{\alpha} \theta^{\beta}=-\frac{1}{2} \epsilon^{\alpha \beta} \theta \theta, & \theta^{\dagger \dot{\alpha}} \theta^{\dagger} \dot{\beta}=\frac{1}{2} \epsilon^{\dot{\alpha} \dot{\beta}} \theta^{\dagger} \theta^{\dagger}, \\
\theta_{\alpha} \theta_{\beta}=\frac{1}{2} \epsilon_{\alpha \beta} \theta \theta, & \theta_{\dot{\alpha}}^{\dagger} \theta_{\dot{\beta}}^{\dagger}=-\frac{1}{2} \epsilon_{\dot{\alpha} \dot{\beta}} \theta^{\dagger} \theta^{\dagger},
\end{array}
$$

where $\theta \theta \equiv \theta^{\alpha} \theta_{\alpha}$ and $\theta^{\dagger} \theta^{\dagger} \equiv \theta_{\dot{\alpha}}^{\dagger} \theta^{\dagger} \dot{\alpha}$ following the convention of eq. (2.33). Products such as $\theta_{\alpha} \theta_{\beta} \theta_{\gamma}=0$, since the spinor indices can assume at most two different values. Finally, the following three results are noteworthy (see Problem 17),

$$
\begin{aligned}
\left(\theta \sigma^{\mu} \theta^{\dagger}\right) \theta_{\beta} & =-\frac{1}{2} \theta \theta\left(\sigma^{\mu} \theta^{\dagger}\right)_{\beta} \\
\left(\theta \sigma^{\mu} \theta^{\dagger}\right) \theta_{\dot{\beta}}^{\dagger} & =-\frac{1}{2} \theta^{\dagger} \theta^{\dagger}\left(\theta \sigma^{\mu}\right)_{\dot{\beta}} \\
\left(\theta \sigma^{\mu} \theta^{\dagger}\right)\left(\theta \sigma^{\nu} \theta^{\dagger}\right) & =\frac{1}{2} g^{\mu \nu}(\theta \theta)\left(\theta^{\dagger} \theta^{\dagger}\right) .
\end{aligned}
$$


Sometimes, we shall write $\theta \theta \theta^{\dagger} \theta^{\dagger} \equiv(\theta \theta)\left(\theta^{\dagger} \theta^{\dagger}\right)$. In such products, there should be no ambiguity in omitting the parentheses.

The Taylor series expansion of a complex superfield $\Phi\left(x, \theta, \theta^{\dagger}\right)$ is therefore given by,

$$
\begin{aligned}
\Phi\left(x, \theta, \theta^{\dagger}\right)= & f(x)+\theta \zeta(x)+\theta^{\dagger} \chi^{\dagger}(x)+\theta \theta m(x)+\theta^{\dagger} \theta^{\dagger} n(x)+\theta \sigma^{\mu} \theta^{\dagger} V_{\mu}(x) \\
& +(\theta \theta) \theta^{\dagger} \lambda^{\dagger}(x)+\left(\theta^{\dagger} \theta^{\dagger}\right) \theta \lambda(x)+\theta \theta \theta^{\dagger} \theta^{\dagger} d(x),
\end{aligned}
$$

where $f, m, n, V_{\mu}$, and $d$ are complex commuting bosonic fields and $\zeta, \chi$, $\lambda$ and $\psi$ are anticommuting two-component fermionic fields. The SUSY transformation laws of the component fields can now be easily obtained (see Problem 18) by comparing both sides of eq. (5.30).

Hence, there are 16 bosonic and 16 fermionic real degrees of freedom. If we impose the constraint, $\Phi^{\dagger}=\Phi$, then $f, d$ and $V_{\mu}$ are real bosonic fields, $n^{\dagger}=m, \zeta=\chi$ and $\lambda=\psi$. In this case, there are 8 bosonic and 8 fermionic real degrees of freedom. In both cases, there are too many degrees of freedom to describe the supermultiplet of the Wess-Zumino model. This is because an unconstrained complex superfield, $\Phi\left(x, \theta, \theta^{\dagger}\right)$, describes a reducible representation of the SUSY algebra. One must impose supersymmetric constraints to project out an irreducible supermultiplet. ${ }^{28}$

The superfield defined in eq. (5.34) is an example of a bosonic superfield, where the Taylor series coefficients of terms even in the number of Grassmann coordinates are commuting bosonic fields and the coefficients of terms odd in the number of Grassmann coordinates are anticommuting fermionic fields. Similarly, one can define a fermionic superfield, where the Taylor series coefficients of terms even in the number of Grassmann coordinates are anticommuting fermionic fields and the coefficients of terms odd in the number of Grassmann coordinates are commuting bosonic fields.

\subsection{Spinor covariant derivatives}

For a superfield $\Phi$, it is easy to check that neither $\partial_{\alpha} \Phi$ nor $\partial_{\dot{\alpha}} \Phi$ is a superfield, since

$$
\partial_{\alpha}\left(\delta_{\xi} \Phi\right) \neq \delta_{\xi}\left(\partial_{\alpha} \Phi\right), \quad \partial_{\dot{\alpha}}^{\dagger}\left(\delta_{\xi} \Phi\right) \neq \delta_{\xi}\left(\partial_{\dot{\alpha}}^{\dagger} \Phi\right) .
$$

Note that if $\Phi$ is a bosonic superfield, then the hermitian conjugate of $\partial_{\alpha} \Phi$ is given by,

$$
\left(\partial_{\alpha} \Phi\right)^{\dagger}=-\partial_{\dot{\alpha}}^{\dagger} \Phi^{\dagger}
$$

where the minus sign above is related to the minus sign in eq. (5.5).

${ }^{28} \mathrm{~A}$ real superfield $\Phi$ yields an off-shell irreducible representation with superspin $j=\frac{1}{2}$. More on this in Section 6. 
We therefore introduce spinor covariant derivatives $D_{\alpha}$ and $\bar{D}_{\dot{\alpha}}$ such that $D_{\alpha} \Phi$ and $\bar{D}_{\dot{\alpha}} \Phi$ are superfields, ${ }^{29}$ which implies the following conditions must be satisfied,

$$
D_{\alpha}\left(\delta_{\xi} \Phi\right)=\delta_{\xi}\left(D_{\alpha} \Phi\right), \quad \bar{D}_{\dot{\alpha}}\left(\delta_{\xi} \Phi\right)=\delta_{\xi}\left(\bar{D}_{\dot{\alpha}} \Phi\right) .
$$

Using eq. (5.30) to express $\delta_{\xi} \Phi$ in terms of the operators $\widehat{Q}$ and $\widehat{Q}^{\dagger}$ defined in eqs. (5.25) and (5.26), respectively, one easily derives

$$
\left\{D_{\alpha}, \widehat{Q}_{\beta}\right\}=\left\{D_{\alpha}, \widehat{Q}_{\dot{\beta}}^{\dagger}\right\}=\left\{\bar{D}_{\dot{\alpha}}, \widehat{Q}_{\beta}\right\}=\left\{\bar{D}_{\dot{\alpha}}, \widehat{Q}_{\dot{\beta}}^{\dagger}\right\}=0 .
$$

To fix the explicit forms for the spinor covariant derivatives, we choose the normalization of $D_{\alpha}$ so that it has the form $D_{\alpha}=\partial_{\alpha}+\ldots$, where the ellipsis refers to correction terms needed to satisfy eqs. (5.37) and (5.38). In the case of $\bar{D}_{\alpha}$, it is customary to impose the condition,

$$
\left(D_{\alpha} \Phi\right)^{\dagger}=\bar{D}_{\dot{\alpha}} \Phi^{\dagger}
$$

where $\Phi$ is a bosonic superfield, in which case $\bar{D}_{\dot{\alpha}}=-\partial_{\dot{\alpha}}^{\dagger}+\ldots$. [cf. eq. (5.36)].

The explicit forms for the spinor covariant derivatives that satisfy the above conditions are given by,

$$
\begin{aligned}
& D_{\alpha}=\partial_{\alpha}-i\left(\sigma^{\mu} \theta^{\dagger}\right)_{\alpha} \partial_{\mu}, \\
& \bar{D}_{\dot{\alpha}}=-\partial_{\dot{\alpha}}^{\dagger}+i\left(\theta \sigma^{\mu}\right)_{\dot{\alpha}} \partial_{\mu} .
\end{aligned}
$$

In particular, $D$ and $\bar{D}$ satisfy the same anticommutation relations as $\widehat{Q}$ and $\widehat{Q}^{\dagger}$ (see Problem 21),

$$
\left\{D_{\alpha}, D_{\beta}\right\}=\left\{\bar{D}_{\dot{\alpha}}, \bar{D}_{\dot{\beta}}\right\}=0 \text { and }\left\{D_{\alpha}, \bar{D}_{\dot{\beta}}\right\}=2 i \sigma_{\alpha \dot{\beta}}^{\mu} \partial_{\mu} .
$$

One can also define spinor covariant derivatives with a raised spinor index. In this case, it is conventional to define,

$$
\begin{aligned}
& D^{\alpha} \equiv \epsilon^{\alpha \beta} D_{\beta}=-\partial^{\alpha}+i\left(\theta^{\dagger} \bar{\sigma}^{\mu}\right)^{\alpha} \partial_{\mu}, \\
& \bar{D}^{\dot{\alpha}} \equiv \epsilon^{\dot{\alpha} \dot{\beta}} D_{\beta}=\partial^{\dagger \dot{\alpha}}-i\left(\bar{\sigma}^{\mu} \theta\right)^{\dot{\alpha}} \partial_{\mu},
\end{aligned}
$$

where we have employed eq. (5.12). That is, the spinor indices of $D_{\alpha}$ and $\bar{D}_{\dot{\alpha}}$ are raised in the conventional way according to eq. (2.24). ${ }^{30}$ The following differential operators will be useful later in these lectures,

$$
\begin{aligned}
& D^{2}=D^{\alpha} D_{\alpha}=-\partial^{\alpha} \partial_{\alpha}+2 i\left(\partial^{\alpha} \sigma_{\alpha \dot{\beta}}^{\mu} \theta^{\dagger \dot{\beta}}\right) \partial_{\mu}+\theta^{\dagger} \theta^{\dagger} \square, \\
& \bar{D}^{2}=\bar{D}_{\dot{\alpha}} \bar{D}^{\dot{\alpha}}=-\partial_{\dot{\alpha}}^{\dagger} \partial^{\dagger \dot{\alpha}}+2 i\left(\theta^{\alpha} \sigma_{\alpha \dot{\beta}}^{\mu} \partial^{\dagger \dot{\beta}}\right) \partial_{\mu}+\theta \theta \square,
\end{aligned}
$$

${ }^{29}$ Note that if $\Phi$ is a bosonic superfield, then $D_{\alpha} \Phi$ and $\bar{D}_{\dot{\alpha}} \Phi$ are fermionic superfields. ${ }^{30}$ This is in contrast to the rule for raising the spinor indices of $\partial_{\alpha}$ and $\partial_{\dot{\alpha}}^{\dagger}$ specified in eq. (5.12), where an extra minus sign appears. 
where $\square \equiv \partial_{\mu} \partial^{\mu}$. One can then derive the following identity (see Problem 22),

$$
\left[D^{2}, \bar{D}^{2}\right]=4 i \sigma_{\alpha \dot{\beta}}^{\mu} \partial_{\mu}\left[D^{\alpha}, \bar{D}^{\dot{\beta}}\right] .
$$

We have employed different notation for the conjugation of the various differential operators that appear in this subsection. The relation of $\widehat{Q}^{\dagger}$ to $\widehat{Q}$ is hermitian conjugation in the same sense that $\hat{P}_{\mu}=i \partial_{\mu}$ [defined in eq. (5.24)] is an hermitian operator in quantum field theory with respect to the inner product defined by the integration of complex fields over spacetime. That is, the dagger on the differential operator $\widehat{Q}^{\dagger}$ denotes Hermitian conjugation with respect to the inner product defined by the integration of complex superfields over superspace. ${ }^{31}$

In contrast, the relation of $\bar{D}$ to $D$ is complex conjugation in the same sense that $\partial_{\mu}^{*}$ is the complex conjugate of $\partial_{\mu}$. In the latter case, the differential operator $\partial_{\mu}$ is a real operator. That is, if we define $\partial_{\mu}^{*}$ to be the derivative operator that acts on the field $\phi$ such that

$$
\left(\partial_{\mu} \phi\right)^{\dagger}=\partial_{\mu}^{*} \phi^{\dagger}
$$

then since $\left(\partial_{\mu} \phi\right)^{\dagger}=\partial_{\mu} \phi^{\dagger}$, it follows that $\partial_{\mu}^{*}=\partial_{\mu}$. In light of eq. (5.39), we can therefore regard $\bar{D}$ as the complex conjugate of $D$.

\subsection{Chiral superfields}

A chiral superfield is obtained by imposing the constraint $\bar{D}_{\dot{\alpha}} \Phi=0$ on a general superfield $\Phi$. Such a constraint is covariant with respect to SUSY transformations, and the end result is an irreducible superfield that corresponds to the superspin $j=0$ irreducible representation of the SUSY algebra. Using eq. (5.41), the constraint yields a differential equation,

$$
\bar{D}_{\dot{\alpha}} \Phi=\left[-\partial_{\dot{\alpha}}^{\dagger}+i\left(\theta \sigma^{\mu}\right)_{\dot{\alpha}} \partial_{\mu}\right] \Phi\left(x, \theta, \theta^{\dagger}\right)=0,
$$

whose solution is of the form

$$
\Phi\left(x, \theta, \theta^{\dagger}\right)=\exp \left(-i \theta \sigma^{\mu} \theta^{\dagger} \partial_{\mu}\right) \Phi(x, \theta) .
$$

We can expand $\Phi(x, \theta)$ in a (truncated) Taylor series in $\theta$,

$$
\Phi(x, \theta)=A(x)+\sqrt{2} \theta \psi(x)+\theta \theta F(x),
$$

where the factor of $\sqrt{2}$ is conventional. Plugging this into eq. (5.50) and using the identity (see Problem 23),

$$
\exp \left(-i \theta \sigma^{\mu} \theta^{\dagger} \partial_{\mu}\right)=1-i \theta \sigma^{\mu} \theta^{\dagger} \partial_{\mu}-\frac{1}{4}(\theta \theta)\left(\theta^{\dagger} \theta^{\dagger}\right) \square
$$

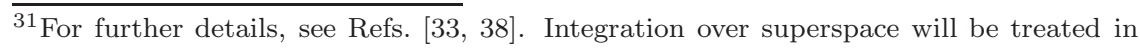
Section 5.7. 
we find after some algebraic manipulation a chiral superfield with the form,

$$
\begin{aligned}
\Phi\left(x, \theta, \theta^{\dagger}\right)= & A(x)+\sqrt{2} \theta \psi(x)+\theta \theta F(x)-i \theta \sigma^{\mu} \theta^{\dagger} \partial_{\mu} A(x) \\
& -\frac{i}{\sqrt{2}}(\theta \theta) \theta^{\dagger} \bar{\sigma}^{\mu} \partial_{\mu} \psi(x)-\frac{1}{4}(\theta \theta)\left(\theta^{\dagger} \theta^{\dagger}\right) \square A(x) .
\end{aligned}
$$

Note that the chiral superfield $\Phi$ has dimension $[\Phi]=1$, in which case it follows that the dimensions of the component fields are $[A]=1$ and $[\psi]=\frac{3}{2}$, as expected, whereas $[F]=2$ after making use of the dimensions of the Grassmann coordinates, $[\theta]=\left[\theta^{\dagger}\right]=-\frac{1}{2}$.

Given a chiral superfield $\Phi$, its hermitian conjugate, $\Phi^{\dagger}$, is an antichiral superfield, which is defined by the SUSY-covariant constraint, $D_{\alpha} \Phi^{\dagger}=0$. Using eq. (5.40), the latter constraint yields a differential equation,

$$
D_{\alpha} \Phi^{\dagger}=\left[\partial_{\alpha}-i\left(\sigma^{\mu} \theta^{\dagger}\right)_{\alpha} \partial_{\mu}\right] \Phi^{\dagger}\left(x, \theta, \theta^{\dagger}\right)=0,
$$

whose solution is of the form

$$
\Phi^{\dagger}\left(x, \theta, \theta^{\dagger}\right)=\exp \left(i \theta \sigma^{\mu} \theta^{\dagger} \partial_{\mu}\right) \Phi^{\dagger}\left(x, \theta^{\dagger}\right) .
$$

We can expand $\Phi^{\dagger}\left(x, \theta^{\dagger}\right)$ in a (truncated) Taylor series in $\theta^{\dagger}$,

$$
\Phi^{\dagger}\left(x, \theta^{\dagger}\right)=A^{\dagger}(x)+\sqrt{2} \theta^{\dagger} \psi^{\dagger}(x)+\theta^{\dagger} \theta^{\dagger} F^{\dagger}(x) .
$$

Plugging this result into eq. (5.55) and following the same procedure as before, we end up with,

$$
\begin{aligned}
\Phi^{\dagger}\left(x, \theta, \theta^{\dagger}\right)= & A^{\dagger}(x)+\sqrt{2} \theta^{\dagger} \psi^{\dagger}(x)+\theta^{\dagger} \theta^{\dagger} F^{\dagger}(x)+i \theta \sigma^{\mu} \theta^{\dagger} \partial_{\mu} A^{\dagger}(x) \\
& -\frac{i}{\sqrt{2}}\left(\theta^{\dagger} \theta^{\dagger}\right) \theta \sigma^{\mu} \partial_{\mu} \psi^{\dagger}(x)-\frac{1}{4}(\theta \theta)\left(\theta^{\dagger} \theta^{\dagger}\right) \square A^{\dagger}(x) .
\end{aligned}
$$

Since $\Phi^{\dagger}$ is the hermitian conjugate of $\Phi$, we can identify $A^{\dagger}, \psi^{\dagger}$ and $F^{\dagger}$ as the hermitian conjugates of $A, \psi$ and $F$.

In calculations, it is often simpler to employ the so-called chiral representation, in which all superfield operators $\mathcal{O}$ are modified according to

$$
\mathcal{O}_{\text {chiral }}=\exp \left(i \theta \sigma^{\mu} \theta^{\dagger} \partial_{\mu}\right) \mathcal{O} \exp \left(-i \theta \sigma^{\mu} \theta^{\dagger} \partial_{\mu}\right) .
$$

In the chiral representation,

$$
\begin{array}{ll}
\widehat{Q}_{\alpha}=i \partial_{\alpha}, & \widehat{Q}_{\dot{\alpha}}^{\dagger}=-i \partial_{\dot{\alpha}}^{\dagger}+2\left(\theta \sigma^{\mu}\right)_{\dot{\alpha}} \partial_{\mu}, \\
\bar{D}_{\dot{\alpha}}=-\partial_{\dot{\alpha}}^{\dagger}, & D_{\alpha}=\partial_{\alpha}-2 i\left(\sigma^{\mu} \theta^{\dagger}\right)_{\alpha} \partial_{\mu} .
\end{array}
$$

Thus, in the chiral representation, the requirement $\bar{D}_{\dot{\alpha}} \Phi=-\partial_{\dot{\alpha}}^{\dagger} \Phi=0$ is simply the requirement that $\Phi$ is independent of $\theta^{\dagger}$. In the chiral representation, the chiral superfield will be denoted by

$$
\Phi_{1}(x, \theta)=A(x)+\sqrt{2} \theta \psi(x)+\theta \theta F(x) .
$$


It then follows that the general expression for a chiral superfield is

$$
\Phi\left(x, \theta, \theta^{\dagger}\right)=\exp \left(-i \theta \sigma^{\mu} \theta^{\dagger} \partial_{\mu}\right) \Phi_{1}(x, \theta)=\Phi_{1}\left(x-i \theta \sigma^{\mu} \theta^{\dagger}, \theta\right) .
$$

It is convenient to define the shifted spacetime coordinate,

$$
y \equiv x-i \theta \sigma^{\mu} \theta^{\dagger},
$$

so that the chiral superfield is given by,

$$
\Phi\left(x, \theta, \theta^{\dagger}\right)=\Phi_{1}(y, \theta) .
$$

The SUSY transformation laws for the fields that appear in the chiral superfield can now be determined simply by inserting the expression for $\Phi$ in the chiral representation given by eq. (5.61) into eq. (5.30). In performing the computation, one employs the chiral representation expressions for $\widehat{Q}$ and $\widehat{Q}^{\dagger}$ given in eq. (5.59). You may verify (see Problem 24) that the result of this calculation coincides with the SUSY transformation laws given previously in eqs. (4.134)-(4.136).

Likewise, one can define an antichiral representation in which

$$
\mathcal{O}_{\text {antichiral }}=\exp \left(-i \theta \sigma^{\mu} \theta^{\dagger} \partial_{\mu}\right) \mathcal{O} \exp \left(i \theta \sigma^{\mu} \theta^{\dagger} \partial_{\mu}\right) .
$$

In the antichiral representation,

$$
\begin{array}{lrl}
\widehat{Q}_{\dot{\alpha}}^{\dagger} & =-i \partial_{\dot{\alpha}}^{\dagger}, & \widehat{Q}_{\alpha}=i \partial_{\alpha}-2\left(\sigma^{\mu} \theta^{\dagger}\right)_{\alpha} \partial_{\mu}, \\
D_{\alpha}=\partial_{\alpha}, & \bar{D}_{\dot{\alpha}}=-\partial_{\dot{\alpha}}^{\dagger}+2 i\left(\theta \sigma^{\mu}\right)_{\dot{\alpha}} \partial_{\mu} .
\end{array}
$$

Thus, in the antichiral representation, the requirement $D_{\alpha} \Phi^{\dagger}=\partial_{\alpha} \Phi^{\dagger}=0$ is simply the requirement that $\Phi^{\dagger}$ is independent of $\theta$. In the antichiral representation, the antichiral superfield will be denoted by

$$
\Phi_{2}\left(x, \theta^{\dagger}\right)=A^{\dagger}(x)+\sqrt{2} \theta^{\dagger} \psi^{\dagger}(x)+\theta^{\dagger} \theta^{\dagger} F^{\dagger}(x) .
$$

It then follows that the general expression for an antichiral superfield is

$$
\Phi^{\dagger}\left(x, \theta, \theta^{\dagger}\right)=\exp \left(i \theta \sigma^{\mu} \theta^{\dagger} \partial_{\mu}\right) \Phi_{2}\left(x, \theta^{\dagger}\right)=\Phi_{2}\left(x+i \theta \sigma^{\mu} \theta^{\dagger}, \theta^{\dagger}\right) .
$$

It is convenient to define the shifted spacetime coordinate,

$$
y^{\dagger} \equiv x+i \theta \sigma^{\mu} \theta^{\dagger},
$$

so that the antichiral superfield is given by,

$$
\Phi^{\dagger}\left(x, \theta, \theta^{\dagger}\right)=\Phi_{2}\left(y^{\dagger}, \theta^{\dagger}\right) .
$$




\subsection{Constructing the SUSY Lagrangian}

\subsubsection{F-terms}

Ultimately, our goal is to construct an action that is invariant under SUSY. It is therefore sufficient to construct a Lagrangian that transforms under SUSY as a total derivative. In the literature, it is common to use the nomenclature $F$-term to denote the coefficient of the $\theta \theta$ term of a superfield. This is sometimes explicitly indicated as follows,

$$
[\Phi]_{\theta \theta}=[\Phi]_{F}=F .
$$

Recall that in eq. (4.136), we demonstrated that the auxiliary field $F(x)$ transforms as a total derivative under the SUSY transformation laws. But, this field is simply the coefficient of the $\theta \theta$ term of a chiral superfield! Indeed, the $F$-term of any chiral superfield transforms under a SUSY transformation as a total derivative. This means that such terms (and their hermitian conjugates) are candidates for terms in a Lagrangian, which then yields an action that is invariant under SUSY.

To discover the relevant $F$-terms for constructing a SUSY Lagrangian, we first prove an important theorem.

Theorem 1. For any positive integers $n$ and $m$, if $\Phi$ is a chiral superfield, then so is $\Phi^{n}$, whereas $\Phi^{n}\left(\Phi^{\dagger}\right)^{m}$ is not a chiral superfield.

Proof. We first note that

$$
\bar{D}_{\dot{\alpha}} \Phi^{n}=n \Phi^{n-1} \bar{D}_{\dot{\alpha}} \Phi=0,
$$

which shows $\Phi^{n}$ satisfies the defining constraint of a chiral superfield. A similar computation shows that $\Phi^{n}\left(\Phi^{\dagger}\right)^{m}$ does not satisfy the required constraint.

An important consequence of the above theorem is that

$$
\sum_{n \geq 1}\left[a_{n} \Phi^{n}\right]_{F}+\text { h.c. }
$$

is a Lorentz scalar that transforms as a total divergence, and thus is a candidate for terms in a Lagrangian whose action is invariant under SUSY.

\subsubsection{Kinetic terms}

To construct the kinetic terms of the SUSY Lagrangian, we define the operator $T$,

$$
T \Phi=-\frac{1}{4} \bar{D}^{2} \Phi^{\dagger},
$$


where $\bar{D}^{2} \equiv \bar{D}_{\dot{\alpha}} \bar{D}^{\dot{\alpha}}$. Note that $\bar{D}_{\dot{\alpha}}(T \Phi)=0$ (due to the anticommutation relations satisfied by $\bar{D}$ ), so that $T \Phi$ is a chiral superfield. In the chiral representation, with $\Phi=A+\sqrt{2} \theta \psi+\theta \theta F$,

$$
T \Phi=F^{\dagger}-i \sqrt{2} \theta \sigma^{\mu} \partial_{\mu} \psi^{\dagger}-\theta \theta \square A^{\dagger} .
$$

Hence, the $F$-component of $\Phi T \Phi$ is given by,

$$
\begin{aligned}
{[\Phi T \Phi]_{F} } & =-A \square A^{\dagger}+F^{\dagger} F+i \psi \sigma^{\mu} \partial_{\mu} \psi^{\dagger} \\
& =\left(\partial_{\mu} A\right)\left(\partial^{\mu} A^{\dagger}\right)+F^{\dagger} F+i \psi^{\dagger} \bar{\sigma}^{\mu} \partial_{\mu} \psi+\text { total derivative, }
\end{aligned}
$$

which we recognize as the kinetic energy term of the Wess-Zumino Lagrangian [cf. eq. (4.130)].

\subsubsection{Mass terms}

To construct the mass terms of the SUSY Lagrangian, the following theorem is useful.

Theorem 2. For any chiral superfield $\Phi$,

$$
[\Phi]_{F}=-\left.\frac{1}{4} D^{2} \Phi\right|_{\theta=\theta^{\dagger}=0}=\left.\frac{1}{4} \partial^{\alpha} \partial_{\alpha} \Phi\right|_{\theta=\theta^{\dagger}=0} .
$$

Proof. Eq. (5.77) follows immediately from eq. (5.45).

We can compute the $F$ term of any holomorphic function of a chiral superfield, $W(\Phi)$, as follows. After making judicious use of the chain rule,

$$
\begin{aligned}
{[W(\Phi)]_{F} } & =\left.\frac{1}{4} \partial^{\alpha} \partial_{\alpha} W\right|_{\theta=\theta^{\dagger}=0}=\left.\frac{1}{4} \partial^{\alpha} \frac{d W}{d \Phi} \partial_{\alpha} \Phi\right|_{\theta=\theta^{\dagger}=0} \\
& =\left.\frac{1}{4}\left\{\left(\frac{d^{2} W}{d \Phi^{2}} \partial^{\alpha} \Phi \partial_{\alpha} \Phi\right)+\frac{d W}{d \Phi} \partial^{\alpha} \partial_{\alpha} \Phi\right\}\right|_{\theta=\theta^{\dagger}=0} .
\end{aligned}
$$

Noting that $\left(\partial^{\alpha} \Phi \partial_{\alpha} \Phi\right)_{\theta=\theta^{\dagger}=0}=-2 \psi \psi$, eq. (5.78) yields,

$$
[W(\Phi)]_{F}=-\frac{1}{2}\left(\frac{d^{2} W}{d \Phi^{2}}\right)_{\Phi=A} \psi \psi+\left(\frac{d W}{d \Phi}\right)_{\Phi=A} F .
$$

Introducing the notation, $d W / d A \equiv(d W / d \Phi)_{\Phi=A}$, it follows that

$$
[W(\Phi)]_{F}=-\frac{1}{2} \frac{d^{2} W}{d A^{2}} \psi \psi+\frac{d W}{d A} F .
$$

In the jargon of SUSY, $W(\Phi)$ is called the superpotential. For renormalizable theories, $W(\Phi)$ is at most cubic in $\Phi$. 


\subsubsection{The Wess-Zumino SUSY Lagrangian using F-terms}

Collecting the results of eqs. (5.76) and (5.80), we end up with,

$$
\begin{aligned}
& \mathscr{L}=[\Phi T \Phi]_{F}+\left\{[W(\Phi)]_{F}+\text { h.c. }\right\} \\
&=\left(\partial_{\mu} A\right)^{\dagger}\left(\partial^{\mu} A\right)+i \psi^{\dagger} \bar{\sigma}^{\mu} \partial_{\mu} \psi+F \frac{d W}{d A}+F^{\dagger}\left(\frac{d W}{d A}\right)^{\dagger}+F^{\dagger} F \\
&-\frac{1}{2}\left[\frac{d^{2} W}{d A^{2}} \psi \psi+\left(\frac{d^{2} W}{d A^{2}}\right)^{\dagger} \psi^{\dagger} \psi^{\dagger}\right]
\end{aligned}
$$

after dropping total derivative terms. We have thus recovered the WessZumino Lagrangian that was previously written down in eq. (4.109).

The proof that the Wess-Zumino action is supersymmetric, or equivalently, $\delta_{\xi} \mathcal{L}=\partial_{\mu} K^{\prime \mu}$, is now trivial since $\mathscr{L}$ was constructed from $F$-terms, which transform as total derivatives under SUSY transformations.

\subsubsection{An alternate form for the kinetic terms: D-terms and the Kähler potential}

The approach of subsection 5.5.2 is not the only supersymmetric way to construct the kinetic energy terms. Consider an unconstrained superfield $V\left(x, \theta, \theta^{\dagger}\right)$. Expanding $V$ as a Taylor series in $\theta$ and $\theta^{\dagger}$, the highest order nonvanishing term is proportional to $(\theta \theta)\left(\theta^{\dagger} \theta^{\dagger}\right)$. If we write

$$
V\left(x, \theta, \theta^{\dagger}\right)=\cdots+(\theta \theta)\left(\theta^{\dagger} \theta^{\dagger}\right) D(x),
$$

then one can show that $\delta_{\xi} D(x)$ is a total derivative using dimensional analysis as we did for $\delta_{\xi} F(x)$ at the end of Section 4.6. Hence, $D$-terms can also provide suitable terms for a SUSY Lagrangian.

We shall denote the $D$-term by,

$$
[V]_{\theta \theta \theta^{\dagger} \theta^{\dagger}}=[V]_{D}=D
$$

using a notation analogous to that of eq. (5.71). The relevant theorem analogous to eq. (5.77) is given below.

Theorem 3. For any superfield $V$,

$$
[V]_{D}=\left.\frac{1}{16} \bar{D}^{2} D^{2} V\right|_{\theta=\theta^{\dagger}=0}=\left.\frac{1}{16}\left(\partial_{\dot{\alpha}}^{\dagger} \partial^{\dagger \dot{\alpha}}\right)\left(\partial^{\alpha} \partial_{\alpha}\right) V\right|_{\theta=\theta^{\dagger}=0} .
$$

Proof. Eq. (5.84) follows immediately from eqs. (5.45) and (5.46). 
For example, if $\Phi$ is a chiral superfield, one can show that (see Problem 25),

$$
\left[\Phi^{\dagger} \Phi\right]_{D}=\left(\partial_{\mu} A\right)\left(\partial^{\mu} A^{\dagger}\right)+F^{\dagger} F+i \psi^{\dagger} \bar{\sigma}^{\mu} \partial_{\mu} \psi+\text { total derivative, }
$$

which again reproduces the kinetic energy terms of the Wess-Zumino Lagrangian.

Indeed, one can obtain candidate terms for a SUSY Lagrangian by considering the $\theta \theta \theta^{\dagger} \theta^{\dagger}$ component of an arbitrary function of a chiral superfield and its complex conjugate. This function, denoted by $K\left(\Phi, \Phi^{\dagger}\right)$, is called the Kähler potential. Applying the chain rule as in our computation of $[W(\Phi)]_{F}$ [cf. eqs. (5.78)-(5.80)], one can calculate (see Problem 26),

$$
\begin{aligned}
{\left[K\left(\Phi, \Phi^{\dagger}\right)\right]_{D}=} & \frac{\partial^{2} K}{\partial A \partial A^{\dagger}}\left[\left(\partial_{\mu} A\right)\left(\partial^{\mu} A^{\dagger}\right)+F^{\dagger} F+\frac{1}{2} i \psi^{\dagger} \bar{\sigma}^{\mu} \stackrel{\leftrightarrow}{\partial} \psi\right] \\
& -\frac{1}{2} \frac{\partial^{3} K}{\partial A \partial A^{\dagger}}\left[F \psi^{\dagger} \psi^{\dagger}+i \psi^{\dagger} \bar{\sigma}^{\mu} \psi \partial_{\mu} A^{\dagger}\right] \\
& -\frac{1}{2} \frac{\partial^{3} K}{\partial A^{2} \partial A^{\dagger}}\left[F^{\dagger} \psi \psi-i \psi^{\dagger} \bar{\sigma}^{\mu} \psi \partial_{\mu} A\right] \\
& +\frac{1}{4} \frac{\partial^{4} K}{\partial A^{2} \partial A^{\dagger 2}}(\psi \psi)\left(\psi^{\dagger} \psi^{\dagger}\right)+\text { total derivative }
\end{aligned}
$$

We conclude that the most general SUSY Lagrangian involving a chiral superfield $\Phi$ is given by

$$
\mathscr{L}=\left[K\left(\Phi, \Phi^{\dagger}\right)\right]_{D}+\left\{[W(\Phi)]_{F}+\text { h.c. }\right\} .
$$

The auxiliary field $F$ can be determined via its classical field equation, which yields

$$
F=\left(\frac{\partial^{2} K}{\partial A \partial A^{\dagger}}\right)^{-1}\left[\frac{1}{2} \frac{\partial^{3} K}{\partial A^{2} \partial A^{\dagger}} \psi \psi-\left(\frac{d W}{d A}\right)^{\dagger}\right] .
$$

The case of $K\left(\Phi, \Phi^{\dagger}\right)=\Phi^{\dagger} \Phi$ reduces to the result of eq. (5.85) and corresponds to the kinetic energy term of the Wess-Zumino model as noted above. In this case, eq. (5.88) yields,

$$
F=-\left(\frac{d W}{d A}\right)^{\dagger}
$$

which reproduces the result previously obtained in eq. (4.132).

More complicated Kähler potentials yield non-renormalizable Lagrangians. These arise in low-energy effective field theories (that include operators of dimension greater than four), in supersymmetric $\sigma$-models, and in supergravity. Such applications lie beyond the scope of these lectures. 


\subsection{R-invariance}

Recall that the SUSY algebra can be extended by added adding a bosonic $\mathrm{U}(1)_{R}$ generator $R$ such that [cf. eqs. (4.36)-(4.39)],

$$
\left[R, Q_{\alpha}\right]=-Q_{\alpha}, \quad\left[R, Q_{\dot{\alpha}}^{\dagger}\right]=Q_{\dot{\alpha}}^{\dagger} .
$$

The action of $\mathrm{U}(1)_{R}$ on a superfield $\Phi$ can be represented by a differential operator $\widehat{R}$ acting on superspace,

$$
[\Phi, R]=\widehat{R} \Phi,
$$

where

$$
\widehat{R} \equiv \theta^{\alpha} \partial_{\alpha}-\theta_{\dot{\alpha}}^{\dagger} \partial^{\dagger \dot{\alpha}}-n, \quad \text { with } n \in \mathbb{R} .
$$

We call $n$ the weight (or $R$-charge) of the superfield $\Phi$. (For a real superfield, only $n=0$ is possible.) Under a $\mathrm{U}(1)_{R}$ transformation,

$$
\delta_{a} \Phi=i a[R, \Phi]=-i a \widehat{R} \Phi .
$$

Acting on a superfield $\Phi\left(x, \theta, \theta^{\dagger}\right)$,

$$
\widehat{R} \Phi\left(x, \theta, \theta^{\dagger}\right)=e^{i n a} \Phi\left(x, e^{-i a} \theta, e^{i a} \theta^{\dagger}\right),
$$

The differential operator $\widehat{R}$ satisfies the identities,

$$
\begin{aligned}
D_{\alpha} \widehat{R} & =(\widehat{R}+1) D_{\alpha}, \\
\bar{D}_{\dot{\alpha}} \widehat{R} & =(\widehat{R}-1) \bar{D}_{\dot{\alpha}} .
\end{aligned}
$$

Hence, it follows that if $\Phi$ is a chiral [antichiral] superfield, then $\widehat{R} \Phi$ is a chiral [antichiral] superfield.

Given a chiral superfield, $\Phi=A+\sqrt{2} \theta \psi+\theta \theta F$, in the chiral representation, the $\mathrm{U}(1)_{R}$ transformations of the component fields are:

$$
\begin{aligned}
& A \rightarrow e^{i n a} A, \\
& \psi \rightarrow e^{i(n-1) a} \psi, \\
& F \rightarrow e^{i(n-2) a} F,
\end{aligned}
$$

after employing eq. (5.94).

Theorem 4. The kinetic energy term $\left[\Phi^{\dagger} \Phi\right]_{D}$ is automatically $R$-invariant, whereas $[W(\Phi)]_{F}$ is $R$-invariant if and only if $W$ has $R$-charge equal to 2.

Proof. If $n=2$, then $F$ is invariant under a $\mathrm{U}(1)_{R}$ transformation, in light of eq. (5.99). This result applies to any $F$-term.

Example 7 (Wess-Zumino model with $W(\Phi)=\frac{1}{2} m \Phi^{2}+\frac{1}{3} g \Phi^{3}$ ). If $m=0$, then the Wess-Zumino model is $R$-invariant with $n=\frac{1}{3}$. If $g=0$, then the Wess-Zumino model is $R$-invariant with $n=\frac{1}{2}$. If both $m \neq 0$ and $g \neq 0$, then the Wess-Zumino model is not $R$-invariant. 


\subsection{Grassmann integration and the SUSY action}

A supersymmetric action can be written as an integral over superspace. First, we introduce integration over anticommuting Grassmann variables. The rules of integration are [111],

$$
\int d \theta=\int d \theta^{\dagger}=0, \quad \int \theta d \theta=\int \theta^{\dagger} d \theta^{\dagger}=1 .
$$

That is, integration over Grassmann variables is in some sense equivalent to differentiation.

It is conventional to define

$$
\begin{aligned}
d^{2} \theta & \equiv-\frac{1}{4} d \theta^{\alpha} d \theta_{\alpha}, \\
d^{2} \theta^{\dagger} & \equiv-\frac{1}{4} d \theta_{\dot{\alpha}}^{\dagger} d \theta^{\dagger \dot{\alpha}}, \\
d^{4} \theta & \equiv d^{2} \theta d^{2} \theta^{\dagger},
\end{aligned}
$$

which yields the following non-zero integrals,

$$
\int d^{2} \theta(\theta \theta)=\int d^{2} \theta^{\dagger}\left(\theta^{\dagger} \theta^{\dagger}\right)=\int d^{4} \theta(\theta \theta)\left(\theta^{\dagger} \theta^{\dagger}\right)=1 .
$$

It follows that for a chiral superfield,

$$
\int d^{2} \theta \Phi\left(x, \theta, \theta^{\dagger}\right)=\int d^{2} \theta \Phi_{1}(x, \theta)=[\Phi]_{F}=-\left.\frac{1}{4} D^{2} \Phi\right|_{\theta=\theta^{\dagger}=0} .
$$

Likewise, for an arbitrary superfield $V\left(x, \theta, \theta^{\dagger}\right)$,

$$
\int d^{4} \theta V\left(x, \theta, \theta^{\dagger}\right)=[V]_{D}=\left.\frac{1}{16} \bar{D}^{2} D^{2} V\right|_{\theta=\theta^{\dagger}=0} .
$$

Thus, the most general SUSY action involving a chiral superfield $\Phi$ is

$$
S=\int d^{4} x d^{4} \theta K\left(\Phi, \Phi^{\dagger}\right)+\int d^{4} x d^{2} \theta W(\Phi)+\int d^{4} x d^{2} \theta^{\dagger} W\left(\Phi^{\dagger}\right)
$$

Generalizations to theories with multiple chiral superfields are straightforward. In the more general case, $W$ is a holomorphic multivariable function of the chiral superfields, and $K$ is a multivariable function of the chiral superfields and their hermitian conjugates. For a renormalizable theory, $W$ is at most a cubic multinomial,

$$
W\left(\Phi_{i}\right)=\sum_{i} a_{i} \Phi_{i}+\sum_{i, j} b_{i j} \Phi_{i} \Phi_{j}+\sum_{i, j, k} c_{i j k} \Phi_{i} \Phi_{j} \Phi_{k},
$$

and

$$
K\left(\Phi_{i}, \Phi_{i}^{\dagger}\right)=\sum_{i} \Phi_{i}^{\dagger} \Phi_{i}
$$


In special cases, one can convert an integral over "half" of superspace (e.g. integrals over $d^{4} x d^{2} \theta$ ) into an integral over the full superspace. The key observation is that for an arbitrary superfield $V$,

$$
\int d^{4} x d^{2} \theta V\left(x, \theta, \theta^{\dagger}\right)=\int d^{4} x\left(-\frac{1}{4} D^{2} V\right) .
$$

On the left-hand side of eq. (5.110), the integration over $d^{2} \theta$ projects out all terms proportional to $\theta \theta$. On the right-hand side, $D^{2}=-\partial^{\alpha} \partial_{\alpha}$ up to total derivative terms that can be dropped because we are integrating over $d^{4} x$. Hence, $\frac{1}{4} \partial^{\alpha} \partial_{\alpha}$ has the effect of projecting out all terms proportional to $\theta \theta$. Likewise,

$$
\int d^{4} x d^{2} \theta^{\dagger} V\left(x, \theta, \theta^{\dagger}\right)=\int d^{4} x\left(-\frac{1}{4} \bar{D}^{2} V\right) .
$$

Hence, it follows that

$$
\int d^{4} x d^{2} \theta\left(-\frac{1}{4} \bar{D}^{2} V\right)=\int d^{4} x d^{4} \theta V\left(x, \theta, \theta^{\dagger}\right) .
$$

Eqs. (5.105) and (5.106) identify integrals over half of superspace as $F$-terms and integrals over the full superspace as $D$-terms. However, eq. (5.112) appears to blur the distinction between $D$-terms and $F$-terms. For example, in the Wess-Zumino Lagrangian, the kinetic energy term may be written as an $F$-term, $[\Phi T \Phi]_{F}$ [cf. eq. (5.81)], or as a $D$-term, $\left[\Phi^{\dagger} \Phi\right]_{D}$, as in eqs. (5.85) and (5.87). However, consider the case of a half superspace integral of the superpotential given in eq. (5.107). If we attempt to convert this into a full superspace integral using eq. (5.112), the end result is

$$
\int d^{4} x d^{2} \theta W(\Phi)=-4 \int d^{4} x d^{4} \theta \bar{D}^{-2} W(\Phi) .
$$

Due to the inverse differential operator, the integrand on the right-hand side of eq. (5.113) is a non-local functional of chiral superfields. This provides the distinction between $F$-terms and $D$-terms. In particular, any half superspace integral that can be converted into a full superspace integral over a local functional of superfields will be called a $D$-term.

Having written the action in eq. (5.107) as an integral over superspace (for $D$-terms) and half of superspace (for $F$-terms), one can obtain expressions for the Green functions of quantum chiral (and antichiral) superfields. The corresponding two-point functions provide expressions for the superspace propagators. One can then formulate a set of superspace Feynman rules and develop a diagrammatic representation of the perturbative expansion of the Green functions. This was first carried out by Grisaru, Roček, 
and Siegel [108], and was applied to the perturbative computation of the effective action. Indeed, such techniques are quite useful since a single supergraph (in which individual lines correspond to superfields) is equivalent to a large number of Feynman diagrams involving the corresponding component fields. A comprehensive treatment of these methods are beyond the scope of these lectures. For a pedagogical development of supergraphs and superspace Feynman rules, see e.g. Refs. [3, 4, 11, 112].

\subsection{Improved ultraviolet behavior of supersymmetry}

An attractive feature of supersymmetric quantum field theories is that their ultraviolet divergences are better behaved, as compared to ordinary quantum field theories. Ref. [108] demonstrated that the loop corrections to the effective action of a supersymmetric theory of chiral superfields can be expressed as an integral over the full superspace,

$$
\sum_{n} \int d^{4} x_{1} \cdots d^{4} x_{n} \int d^{4} \theta g_{n}\left(x_{1}, \ldots, x_{n}\right) F_{1}\left(x_{1}, \theta, \theta^{\dagger}\right) \cdots F_{n}\left(x_{n}, \theta, \theta^{\dagger}\right),
$$

where the $F_{i}\left(x_{i}, \theta, \theta^{\dagger}\right)$ are local functionals of chiral and antichiral superfields and their covariant derivatives, and the $g_{n}$ are translationally invariant functions on Minkowski space.

Eq. (5.114) implies that $D$-terms are renormalized but $F$-terms are not renormalized. Moreover, if $F$-terms are absent at tree-level, then they are not generated at the loop level. Hence, the tree-level Kähler potential is renormalized by radiative corrections, whereas there are no loop corrections to the tree-level superpotential. This is the famous non-renormalization theorem of $N=1$ supersymmetry. ${ }^{32}$ The proof of the non-renormalization theorem in Ref. [108] relies on the analysis of supergraphs in perturbation theory, and is beyond the scope of these lectures. Heuristically, this theorem is a consequence of an exact cancellation between fermion and boson loop contributions to the effective action due to supersymmetry.

Note that the non-renormalization of the tree-level superpotential is simply a consequence of the fact that the integral of a product of chiral

\footnotetext{
${ }^{32}$ The proof of the non-renormalization theorem implicitly assumes that the function $g_{n}$ in eq. (5.114) is local. However, the non-renormalization theorem can fail if the supersymmetric theory contains massless fields as shown in Refs. [113-115], due to infrared divergences. For example, the inverse Laplacian operator $\square^{-1}$ (from a massless propagator) can appear, resulting in a non-local function $g_{n}$ in eq. (5.114). One can show that the non-renormalization theorem holds for the Wilsonian effective action [116, 117], where the infrared effects are cut off [109, 118].
} 
superfields over all of superspace in eq. (5.114) is zero due to eq. (5.100) [see Problem 29]. Moreover, the assumption that the $F_{i}$ in eq. (5.114) are local functionals of chiral and antichiral superfields is essential. Otherwise, one could employ eq. (5.113) and erroneously claim the existence of loop corrections to the tree-level superpotential.

We now briefly explore the consequence of the non-renormalization of the superpotential. Consider the action of the Wess-Zumino model,

$$
S_{\mathrm{WZ}}=\int d^{4} x \int d^{4} \theta \Phi^{\dagger} \Phi+\left[\int d^{4} x \int d^{2} \theta\left(\frac{1}{2} m \Phi^{2}+\frac{1}{3} \lambda \Phi^{3}\right)+\text { h.c. }\right] .
$$

The non-renormalization theorem implies that renormalized fields and parameters are related to bare fields and parameters as follows [119],

$$
\Phi_{R}=Z^{-1 / 2} \Phi, \quad m_{R}=Z m, \quad \lambda_{R}=Z^{3 / 2} \lambda .
$$

where the subscript $R$ indicates renormalized quantities and the bare quantities have no subscript. Eq. (5.116) is equivalent to the statement that the superpotential is unrenormalized, $W_{R}\left(\Phi_{R}\right)=W(\Phi)$. That is,

$$
\frac{1}{2} m_{R} \Phi_{R}^{2}+\frac{1}{3} \lambda_{R} \Phi_{R}^{3}=\frac{1}{2} m \Phi^{2}+\frac{1}{3} \lambda \Phi^{3} .
$$

Wave function renormalization is a consequence of the renormalization of the Kähler potential $\left(\Phi^{\dagger} \Phi\right.$ in the case of the Wess-Zumino model).

The non-renormalization theorem does not assert that the parameters of the superpotential are not renormalized. Indeed, eq. (5.116) states that the renormalization of the parameters $m$ and $\lambda$ are governed by the wave function renormalization constant $Z$. Moreover, the wave function renormalization constants of the component fields of the chiral superfield are equal (i.e., $A_{R}=Z^{-1 / 2} A$ and $\psi_{R}=Z^{-1 / 2} \psi$ ), as a consequence of supersymmetry.

In Ref. [109], Seiberg offered a more intuitive understanding of the nonrenormalization theorem, which also forbids nonperturbative corrections to the Wilsonian effective action [cf. footnote 32]. Seiberg's argument draws on the symmetry and holomorphy ${ }^{33}$ of the superpotential. Consider again the example of the Wess-Zumino superpotential, $W(\Phi)=\frac{1}{2} m \Phi^{2}+\frac{1}{3} \lambda \Phi^{3}$. Following Ref. [109], one can think of $m$ and $\lambda$ as the vacuum expectation values of chiral superfields, so that $W$ must be holomorphic in $m$ and $\lambda$ as well as in $\Phi$. In light of Theorem 4 in Section 5.6, the theory is invariant under an enhanced $\mathrm{U}(1) \times \mathrm{U}(1)_{R}$ symmetry, with the charge assignments shown in Table 4.

\footnotetext{
${ }^{33}$ The fact that the superpotential is a holomorphic function of chiral superfields plays a critical role in Seiberg's argument. In contrast, the renormalization of the Kähler potential is possible because the latter is a function of chiral and antichiral superfields and hence is not holomorphic.
} 
Table 4.: Charge assignments under the $\mathrm{U}(1) \times \mathrm{U}(1)_{R}$ symmetry.

\begin{tabular}{|l|rrrr|}
\hline & $\Phi$ & $\Phi^{\dagger}$ & $m$ & $\lambda$ \\
\hline $\mathrm{U}(1)$ & 1 & -1 & -2 & -3 \\
$\mathrm{U}(1)_{R}$ & 1 & 1 & 0 & -1 \\
\hline
\end{tabular}

To maintain the $\mathrm{U}(1) \times U(1)_{R}$ symmetry and holomorphy, corrections to the Wilsonian effective superpotential must therefore be of the form

$$
m \Phi^{2} f\left(\frac{\lambda \Phi}{m}\right)
$$

where $f$ is an arbitrary holomorphic function. Eq. (5.118) is valid for arbitrary $\lambda$. Thus, we can take $|\lambda| \ll 1$, in which case perturbation theory should be valid. Expanding in powers of the coupling constant $\lambda$, the perturbative expansion should have the following form,

$$
W_{\mathrm{eff}}=\sum_{n=0}^{\infty} a_{n} \frac{\lambda^{n}}{m^{n-1}} \Phi^{n+2} .
$$

The terms in $W_{\text {eff }}$ are represented diagrammatically by one particle irreducible (1PI) supergraphs constructed from propagators and three-point vertices proportional to $\lambda$. However, one cannot construct a one-loop (or higher) supergraph that behaves like $\lambda^{n} \Phi^{n+2}$. It is easy to show that treelevel diagrams with $n+2$ external legs, $n$ vertices and $n-1$ propagators would behave like $\lambda^{n} \Phi^{n+2}$. But, the only 1PI tree-level graphs are those with either two or three external legs! Hence, we conclude that $a_{0}=\frac{1}{2}$, $a_{1}=\frac{1}{3}$ and $a_{n}=0$ for $n \geq 2 .{ }^{34}$ That is $W_{\text {eff }}(\Phi)=W_{\text {tree }}(\Phi)$, which is the statement that the superpotential is not renormalized.

\subsection{Problems}

Problem 15. Prove that

$$
G\left(y, \xi, \xi^{\dagger}\right) G\left(x, \theta, \theta^{\dagger}\right)=G\left(x+y+i\left(\xi \sigma \theta^{\dagger}-\theta \sigma \xi^{\dagger}\right), \xi+\theta, \xi^{\dagger}+\theta^{\dagger}\right) .
$$

HINT: use the Baker-Campbell-Hausdorff formula given in eq. (5.15).

Problem 16. Verify that when acting on a superfield $\Phi\left(x, \theta, \theta^{\dagger}\right)$,

$$
\left\{\widehat{Q}_{\alpha}, \widehat{Q}_{\beta}\right\}=\left\{\widehat{Q}_{\dot{\alpha}}^{\dagger}, \widehat{Q}_{\dot{\beta}}^{\dagger}\right\}=0, \quad\left\{\widehat{Q}_{\alpha}, \widehat{Q}_{\dot{\beta}}^{\dagger}\right\}=2 \sigma_{\alpha \dot{\beta}}^{\mu} \widehat{P}_{\mu} .
$$

${ }^{34}$ One can also conclude that $a_{n}=0$ for $n \geq 2$ by noting that the Wilsonian effective action $W_{\text {eff }}$ must have a smooth limit as $m \rightarrow 0$. 
Problem 17. Prove eqs. (5.31)-(5.33). The last result is an example of a Fierz identity (see, e.g., Appendix B of Ref. [1] or Appendix A of Ref. [10]).

Problem 18. Using eq. (5.30), obtain the SUSY transformation laws for the bosonic component fields, $f, m, n, V_{\mu}$, and $d$, and the fermionic component fields, $\zeta, \chi, \lambda$ and $\psi$, which appear in the complex superfield defined in eq. (5.34).

Problem 19. Suppose that $\Phi$ is a bosonic superfield. Verify that eq. (5.36) holds. Then, show that eqs. (5.40) and (5.41) satisfy eq. (5.39).

Problem 20. Suppose that $\Phi$ is a fermionic superfield. Show that eqs. (5.36) and (5.39) are modified as follows: $\left(\partial_{\alpha} \Phi\right)^{\dagger}=\partial_{\dot{\alpha}}^{\dagger} \Phi^{\dagger}$ and $\left(D_{\alpha} \Phi\right)^{\dagger}=-\bar{D}_{\dot{\alpha}} \Phi^{\dagger}$.

Problem 21. Show that the spinor covariant derivatives, as defined in eq. (5.40) and eq. (5.41), satisfy the following anticommutation relations, $\left\{D_{\alpha}, D_{\beta}\right\}=\left\{\bar{D}_{\dot{\alpha}}, \bar{D}_{\dot{\beta}}\right\}=0$ and $\left\{D_{\alpha}, \bar{D}_{\dot{\beta}}\right\}=2 i \sigma_{\alpha \dot{\beta}}^{\mu} \partial_{\mu}$.

Problem 22. Derive eq. (5.47).

Problem 23. Prove that

$$
\exp \left(-i \theta \sigma^{\mu} \theta^{\dagger} \partial_{\mu}\right)=1-i \theta \sigma^{\mu} \theta^{\dagger} \partial_{\mu}-\frac{1}{4}(\theta \theta)\left(\theta^{\dagger} \theta^{\dagger}\right) \square
$$

where $\square \equiv \partial_{\mu} \partial^{\mu}$.

Problem 24. Using eq. (5.30), one can obtain the SUSY transformation laws for the component fields $A, \psi$ and $F$ in eq. (5.53). Perform the calculation by working in the chiral representation and show that the SUSY transformation laws for $A, \psi$ and $F$ coincide with the results obtained previously in eqs. (4.134)-(4.136) for the fields of a superspin $j=0$ supermultiplet.

Problem 25. If $\Phi$ is a chiral superfield, show that

$$
\left[\Phi^{\dagger} \Phi\right]_{D}=\left(\partial_{\mu} A\right)\left(\partial^{\mu} A^{\dagger}\right)+F^{\dagger} F+i \psi^{\dagger} \bar{\sigma}^{\mu} \partial_{\mu} \psi+\text { total derivative } .
$$

Problem 26. Derive eq. (5.86).

Problem 27. A linear superfield [105, 106], $L(x, \theta, \bar{\theta})$, is defined as a constrained real scalar superfield that satisfies, $D^{2} L(x, \theta, \bar{\theta})=\bar{D}^{2} L(x, \theta, \bar{\theta})=0$. Identify the component fields that make up the linear superfield. Show that $\partial_{\mu} V^{\mu}=0$, where $V^{\mu}$ is the component vector field of $L$. Check that the number of fermion and boson degrees of freedom of the linear superfield are equal. [HINT: the identity given by eq. (5.47) should be helpful.] 
Problem 28. Employing the operator $T$ defined in eq. (5.74), show that

$$
\int d^{4} x d^{2} \theta \Phi T \Phi=\int d^{4} x d^{4} \theta \Phi^{\dagger} \Phi,
$$

by converting the integral over half of superspace into an integral over the full superspace. Use the above result to conclude that $[\Phi T \Phi]_{F}=\left[\Phi^{\dagger} \Phi\right]_{D}$.

Problem 29. If $\Phi$ is a chiral superfield and $\Phi^{\dagger}$ is an antichiral superfield, show that

$$
\int d^{4} x d^{4} \theta \Phi\left(x, \theta, \theta^{\dagger}\right)=\int d^{4} x d^{4} \theta \Phi^{\dagger}\left(x, \theta, \theta^{\dagger}\right)=0 .
$$

\section{Supersymmetric gauge theories}

In this section, we discuss the supersymmetric extension of gauge theories. We begin with the vector superfield $V$, which contains the gauge fields as well as their supersymmetric partners, the gauginos. We discuss the behavior of $V$ under a gauge transformation, and the gauge-invariant interaction terms that couple the vector superfield with one or more chiral superfields. Both abelian and non-abelian gauge groups are treated. Finally, we construct the SUSY Lagrangians corresponding to QED and a non-Abelian SUSY Yang-Mill theory coupled to supersymmetric matter.

\subsection{Vector superfields}

Imposing a reality condition on a complex superfield (which is a covariant constraint with respect to SUSY transformations), we obtain the so-called real vector superfield,

$$
V\left(x, \theta, \theta^{\dagger}\right)=V^{\dagger}\left(x, \theta, \theta^{\dagger}\right),
$$

which will be employed in constructing supersymmetric gauge theories. Expanding in $\theta$ and $\theta^{\dagger}$,

$$
\begin{aligned}
V= & C+i \theta \chi-i \theta^{\dagger} \chi^{\dagger}+\frac{1}{2} i \theta \theta(M+i N)-\frac{1}{2} i \theta^{\dagger} \theta^{\dagger}(M-i N)+\theta \sigma^{\mu} \theta^{\dagger} V_{\mu} \\
& +i(\theta \theta) \theta^{\dagger}\left(\lambda^{\dagger}-\frac{1}{2} i \bar{\sigma}^{\mu} \partial_{\mu} \chi\right)-i\left(\theta^{\dagger} \theta^{\dagger}\right) \theta\left(\lambda-\frac{1}{2} i \sigma^{\mu} \partial_{\mu} \chi^{\dagger}\right) \\
& +\frac{1}{2}(\theta \theta)\left(\theta^{\dagger} \theta^{\dagger}\right)\left(D-\frac{1}{2} \square C\right),
\end{aligned}
$$

where $C, M, N, D$ and $V_{\mu}$ are real bosonic fields, and $\chi$ and $\lambda$ are twocomponent fermion fields. The various factors of $i$ and $\frac{1}{2}$ are conventional, and the particular linear combination of fields chosen as coefficients of $(\theta \theta) \theta^{\dagger},\left(\theta^{\dagger} \theta^{\dagger}\right) \theta$ and $(\theta \theta)\left(\theta^{\dagger} \theta^{\dagger}\right)$ are convenient for later purposes [cf. footnote 38 ]. Note that the superfield $V$ is dimensionless, in which case it follows 
that the dimensions of the component fields are $\left[V_{\mu}\right]=1$ and $[\lambda]=\frac{3}{2}$, as expected, whereas $[C]=[D]=0$, and $[\chi]=\frac{1}{2}$ after making use of the dimensions of the Grassmann coordinates, $[\theta]=\left[\theta^{\dagger}\right]=-\frac{1}{2}$.

The real vector field $V_{\mu}$ is a candidate for a gauge boson of an abelian $\mathrm{U}(1)$ gauge theory. The corresponding field strength tensor is given by

$$
F_{\mu \nu}=\partial_{\mu} V_{\nu}-\partial_{\nu} V_{\mu} .
$$

Indeed, this can be shown to be one of the components of the field strength superfield, which is defined by

$$
\mathcal{W}_{\alpha}=-\frac{1}{4} \bar{D}^{2} D_{\alpha} V
$$

Note that $\bar{D}_{\dot{\beta}} \mathcal{W}_{\alpha}=0$, so that $\mathcal{W}_{\alpha}$ is a spinor chiral superfield. Evaluating the above expression, and expressing it in the chiral representation,

$$
\mathcal{W}_{\alpha}\left(y, \theta, \theta^{\dagger}\right)=-i \lambda_{\alpha}+\theta_{\alpha} D-\frac{1}{2} i\left(\sigma^{\mu} \bar{\sigma}^{\nu} \theta\right)_{\alpha} F_{\mu \nu}-\theta \theta\left(\sigma^{\mu} \partial_{\mu} \lambda^{\dagger}\right)_{\alpha},
$$

where $y \equiv x-i \theta \sigma^{\mu} \theta^{\dagger}$. The fermionic partner of the gauge boson, called the gaugino, is represented by the two-component spinor field $\lambda$. Remarkably, the fields $C, M, N$ and $\chi$ that are coefficients in the Taylor expansion of the vector superfield $V$ do not appear in eq. (6.5). The reason for this will become apparent in Section 6.2.

One can work out the SUSY transformation laws of the fields, $\lambda, F_{\mu \nu}$ and $D$, by matching component fields on both sides of the following equation,

$$
\delta_{\xi} \mathcal{W}_{\alpha}=-i\left(\xi \widehat{Q}+\xi^{\dagger} \widehat{Q}^{\dagger}\right) \mathcal{W}_{\alpha}
$$

The end result is

$$
\begin{aligned}
\delta_{\xi} \lambda_{\alpha} & =i \xi_{\alpha} D+\frac{1}{2}\left(\sigma^{\mu} \bar{\sigma}^{\nu}\right)_{\alpha}{ }^{\beta} \xi_{\beta} F_{\mu \nu}, \\
\delta_{\xi} F_{\mu \nu} & =i \partial_{\mu}\left(\xi \sigma_{\nu} \lambda^{\dagger}-\lambda \sigma_{\nu} \xi^{\dagger}\right)-i \partial_{\nu}\left(\xi \sigma_{\mu} \lambda^{\dagger}-\lambda \sigma_{\mu} \xi^{\dagger}\right), \\
\delta_{\xi} D & =\partial_{\mu}\left(\xi \sigma^{\mu} \lambda^{\dagger}+\lambda \sigma^{\mu} \xi^{\dagger}\right) .
\end{aligned}
$$

Note that the mass dimension of the $D$-term is given by $[D]=2$. Hence, dimensional analysis implies that $\delta_{\xi} D$ must be a total derivative, which is confirmed in eq. (6.9). From the above transformation laws, we conclude that $\left(\lambda, \lambda^{\dagger}, F_{\mu \nu}, D\right)$ forms an irreducible supermultiplet (corresponding to superhelicity 1$)$.

To obtain the Lagrangian for the SUSY U(1) gauge theory, note that

$$
\begin{aligned}
\frac{1}{4}\left[\mathcal{W}^{\alpha} \mathcal{W}_{\alpha}\right]_{F}+\text { h.c. } & =\frac{1}{2} i\left(\lambda \sigma^{\mu} \partial_{\mu} \lambda^{\dagger}+\lambda^{\dagger} \bar{\sigma}^{\mu} \partial_{\mu} \lambda\right)+\frac{1}{2} D^{2}-\frac{1}{4} F_{\mu \nu} F^{\mu \nu} \\
& =i \lambda^{\dagger} \bar{\sigma}^{\mu} \partial_{\mu} \lambda+\frac{1}{2} D^{2}-\frac{1}{4} F_{\mu \nu} F^{\mu \nu}+\text { total derivative. }
\end{aligned}
$$


This is the kinetic energy term for a $\mathrm{U}(1)$ gauge field $V_{\mu}$ and its gaugino superpartner $\lambda$. Both the gauge boson and gaugino are massless. The real scalar field $D$ is not dynamical; it is an auxiliary field.

The action corresponding to the Lagrangian of eq. (6.10) can be written as an integral over half of superspace. In particular, eq. (5.105) yields,

$$
\mathscr{L}=\frac{1}{4} \int d^{2} \theta \mathcal{W}^{\alpha} \mathcal{W}_{\alpha}+\text { h.c. }
$$

One can show that $\left[\mathcal{W}^{\alpha} \mathcal{W}_{\alpha}\right]_{F}$ and its hermitian conjugate term differ only by a total derivative. Hence, both terms contribute equally to the action, which is given by

$$
S=\frac{1}{2} \int d^{4} x d^{2} \theta \mathcal{W}^{\alpha} \mathcal{W}_{\alpha}
$$

It is sometimes convenient to turn this integral into an integration over the full superspace. Using a trick analogous to the one employed in eq. (5.112), we end up with,

$$
S=\frac{1}{2} \int d^{4} x d^{2} \theta\left(-\frac{1}{4} \bar{D}^{2}\right)\left(D^{\alpha} V\right) \mathcal{W}_{\alpha}=\frac{1}{2} \int d^{4} x d^{2} \theta d^{2} \theta^{\dagger}\left(D^{\alpha} V\right) \mathcal{W}_{\alpha},
$$

after using eq. (6.4) to rewrite one factor of $\mathcal{W}^{\alpha}$ in terms of $V$.

It is instructive to count the degrees of freedom in the irreducible supermultiplet, $\left(\lambda, \lambda^{\dagger}, F_{\mu \nu}, D\right)$. On-shell, there are two real fermionic degrees of freedom associated with the massless gaugino, after imposing the Lagrange field equations, ${ }^{35}$

$$
i \bar{\sigma}^{\mu \alpha \dot{\beta}} \partial_{\mu} \lambda_{\beta}=0 .
$$

This matches the two real bosonic degrees of freedom corresponding to the two transverse polarizations of the massless gauge boson.

To count the off-shell bosonic degrees of freedom, one must take into account the Bianchi identity, ${ }^{36}$

$$
\epsilon^{\mu \nu \rho \sigma} \partial_{\nu} F_{\rho \sigma}=0
$$

\footnotetext{
${ }^{35}$ Starting with two complex (or equivalently four real) degrees of freedom for the twocomponent gaugino field $\lambda$, eq. (6.14) relates the spinor components $\lambda_{1}$ and $\lambda_{2}$, thereby reducing the number of real degrees of freedom from four to two.

${ }^{36}$ Although it appears that the Bianchi identity yields four constraints, since the spacetime index $\mu$ is a free index, in fact only three constraints are independent. This is because one of the four constraints is redundant due to the identity, $\epsilon^{\mu \nu \rho \sigma} \partial_{\mu} \partial_{\nu} F_{\rho \sigma}=0$, which is automatically satisfied as a result of the antisymmetry of the Levi-Civita tensor. Physically, the Bianchi identity implies that the three components of the electric field vector determine the three components of the magnetic field vector.
} 
which is satisfied independently of the field equations. This identity reduces the number of real degrees of freedom in the real antisymmetric tensor $F_{\mu \nu}$ from six to three. Adding in the one real degree of freedom associated with $D$, we end up with a total of four real bosonic degrees of freedom, which matches the four real off-shell fermionic degrees of freedom corresponding to $\lambda$ and $\lambda^{\dagger}$.

\subsection{Gauge invariance}

The vector superfield $V$ contains the familiar gauge field $V_{\mu}$. But it also includes other component fields $C, \chi, M$ and $N$, whose meaning is less obvious. As we will see, these latter fields turn out to be gauge artifacts. Thus, we must examine how gauge transformations of the gauge field theory get promoted to gauge transformations of the vector superfield $V$.

Let $\Lambda\left(x, \theta, \theta^{\dagger}\right)$ be a chiral superfield (i.e., $\left.\bar{D}_{\dot{\alpha}} \Lambda=0\right)$ and let $\Lambda^{\dagger}\left(x, \theta, \theta^{\dagger}\right)$ be the corresponding antichiral superfield. Consider the transformation,

$$
V \rightarrow V+i\left(\Lambda-\Lambda^{\dagger}\right) \text {. }
$$

We assert that eq. (6.16) is a supersymmetric generalization of the gauge transformation of an abelian gauge theory, henceforth called a super gauge transformation.

With the help of eq. (5.42), it is straightforward to show that the field strength superfield, $\mathcal{W}_{\alpha}$, is invariant under a super gauge transformation. Moreover, if the Taylor series of $\Lambda\left(x, \theta, \theta^{\dagger}\right)$ is written $\mathrm{as}^{37}$

$$
\begin{aligned}
\Lambda\left(x, \theta, \theta^{\dagger}\right)= & \widetilde{A}(x)+\sqrt{2} \theta \widetilde{\psi}(x)+\theta \theta \widetilde{F}(x)-i \theta \sigma^{\mu} \theta^{\dagger} \partial_{\mu} \widetilde{A}(x) \\
& -\frac{i}{\sqrt{2}}(\theta \theta) \theta^{\dagger} \bar{\sigma}^{\mu} \partial_{\mu} \widetilde{\psi}(x)-\frac{1}{4}(\theta \theta)\left(\theta^{\dagger} \theta^{\dagger}\right) \square \widetilde{A}(x),
\end{aligned}
$$

then the impact of the super gauge transformation given by eq. (6.16) on the component fields of $V$ is, ${ }^{38}$

$$
\begin{aligned}
C & \rightarrow C+i\left(\widetilde{A}-\widetilde{A}^{\dagger}\right), \\
\chi & \rightarrow \chi+\sqrt{2} \widetilde{\psi}, \\
M+i N & \rightarrow M+i N+2 \widetilde{F}, \\
V_{\mu} & \rightarrow V_{\mu}+\partial_{\mu}\left(\widetilde{A}+\widetilde{A}^{\dagger}\right), \\
\lambda & \rightarrow \lambda, \\
D & \rightarrow D .
\end{aligned}
$$

$\overline{{ }^{37} \text { In contrast to the chiral superfield }} \Phi$ in eq. (5.53) whose mass dimension is 1 , the chiral superfield $\Lambda$ is dimensionless, as required for consistency in light of eq. (6.16).

38 The invariance of $\lambda$ and $D$ under super gauge transformations is a consequence of the particular choices made for the coefficients of $(\theta \theta) \theta^{\dagger},\left(\theta^{\dagger} \theta^{\dagger}\right) \theta$ and $(\theta \theta)\left(\theta^{\dagger} \theta^{\dagger}\right)$ in eq. (6.2). 
Indeed, under a super gauge transformation, the gauge field $V_{\mu}$ transforms by an ordinary gauge transformation. Moreover the field strength tensor $F_{\mu \nu}=\partial_{\mu} V_{\nu}-\partial_{\nu} V_{\mu}$, the gaugino field $\lambda$, and the auxiliary field $D$ are gauge invariant as one would anticipate (consistent with the fact that the field strength superfield $\mathcal{W}$ is gauge invariant).

One particularly useful gauge choice is to choose $\widetilde{A}, \widetilde{\psi}$ and $\widetilde{F}$ such that

$$
C=\chi=M=N=0 .
$$

This is called the Wess-Zumino (WZ) gauge [120]. The existence of such a gauge implies that the fields $C, \chi, M$, and $N$ are gauge artifacts, as previously stated. The main drawback of the WZ gauge is that it is not a supersymmetric gauge choice. That is, starting from the WZ gauge and performing a SUSY transformation on the component fields of the vector superfield $V$ will yield new component fields that do not satisfy the WZ gauge condition.

The main benefit of the WZ gauge is that it provides enormous simplification in many practical computations. In particular, applying the WZ gauge condition [eq. (6.24)] to the vector superfield given in eq. (6.2),

$$
V_{\mathrm{WZ}}=\theta \sigma^{\mu} \theta^{\dagger} V_{\mu}+i(\theta \theta)\left(\theta^{\dagger} \bar{\lambda}\right)-i\left(\theta^{\dagger} \theta^{\dagger}\right)(\theta \lambda)+\frac{1}{2}(\theta \theta)\left(\theta^{\dagger} \theta^{\dagger}\right) D .
$$

Computing the square of $V_{\mathrm{WZ}}$ with the help of eq. (5.33) yields,

$$
V_{\mathrm{WZ}}^{2}\left(x, \theta, \theta^{\dagger}\right)=\frac{1}{2}(\theta \theta)\left(\theta^{\dagger} \theta^{\dagger}\right) V_{\mu} V^{\mu} .
$$

and $V_{\mathrm{WZ}}^{n}\left(x, \theta, \theta^{\dagger}\right)=0$ for $n=3,4,5, \ldots$ This implies that the Taylor series for the exponential of $V_{\mathrm{WZ}}$ is a finite series and contains only three terms,

$$
\exp \left(2 g V_{\mathrm{WZ}}\right)=1+2 g V_{\mathrm{WZ}}+2 g^{2} V_{\mathrm{WZ}}^{2} .
$$

This result will be especially important when we consider gauge-invariant interactions in Section 6.3.

Finally, we consider the implications of $R$-invariance. Since $V$ is a real superfield, it follows from eq. (5.94) that,

$$
\widehat{R} V\left(x, \theta, \theta^{\dagger}\right)=V\left(x, e^{-i a} \theta, e^{i a} \theta^{\dagger}\right) .
$$

In the Wess-Zumino gauge, the $R$ transformations of the component fields are given by

$$
\begin{aligned}
V_{\mu} & \rightarrow V_{\mu}, \\
\lambda & \rightarrow e^{i a} \lambda, \\
D & \rightarrow D .
\end{aligned}
$$

The Lagrangian of eq. (6.10) for the SUSY gauge theory is invariant under $R$ transformations. In the present context, the presence of $R$-invariance is associated with the chiral symmetry of the massless gaugino. 


\subsection{Gauge-invariant interactions}

Suppose that $\Phi$ is a chiral superfield that is charged under the $\mathrm{U}(1)$ gauge group. Then the gauge transformations of the chiral superfield and the corresponding antichiral superfield are given by,

$$
\Phi \rightarrow e^{-2 i g \Lambda} \Phi, \quad \Phi^{\dagger} \rightarrow e^{2 i g \Lambda^{\dagger}} \Phi^{\dagger},
$$

where $\Lambda$ is the chiral superfield gauge transformation parameter introduced in eq. (6.16). In the presence of gauge interactions, the kinetic energy term for the chiral superfield given by eq. (5.85),

$$
\mathscr{L}_{\mathrm{KE}}=\left[\Phi^{\dagger} \Phi\right]_{D}=\int d^{4} \theta \Phi^{\dagger} \Phi,
$$

is not gauge invariant. But this deficiency is easily repaired. A gaugeinvariant kinetic energy term with respect to the gauge transformations given in eqs. (6.16) and (6.32) is given by,

$$
\mathscr{L}_{\mathrm{KE}}=\left[\Phi^{\dagger} e^{2 g V} \Phi\right]_{D}=\int d^{4} \theta \Phi^{\dagger} e^{2 g V} \Phi .
$$

The proof is left as an exercise (see Problem 31).

Normally, the exponential, $\exp (2 g V)$, would yield an infinite series of terms. But, the series terminates in the Wess-Zumino gauge, as indicated in eq. (6.27), and we get

$$
\begin{aligned}
\mathscr{L}_{\mathrm{KE}}= & \left(\mathcal{D}_{\mu} A\right)\left(\mathcal{D}^{\mu} A\right)^{\dagger}+i \psi^{\dagger} \bar{\sigma}^{\mu} \mathcal{D}_{\mu} \psi+F^{\dagger} F \\
& +i g \sqrt{2}\left(A^{\dagger} \lambda \psi-A \lambda^{\dagger} \psi^{\dagger}\right)+g A A^{\dagger} D+\text { total derivative }
\end{aligned}
$$

where $\mathcal{D}_{\mu} \equiv \partial_{\mu}+i g V_{\mu}$ is the usual gauge-covariant derivative. The presence of the Yukawa interaction of the scalar-fermion-gaugino is especially noteworthy, with a coupling proportional to the gauge coupling $g$. This is a consequence of supersymmetry, which relates the gauge and Yukawa couplings that otherwise would be independent.

Another manifestation of SUSY is revealed when we consider the terms of the Lagrangian involving the auxiliary fields $F$ and $D$. Consider the Lagrangian of the interacting gauge theory that consists of contributions from eqs. (6.10) and (6.35). We can isolate those terms that involve $F$ and $D$ explicitly,

$$
\mathscr{L}=\left\{\frac{1}{4}\left[\mathcal{W}^{\alpha} \mathcal{W}_{\alpha}\right]_{F}+\text { h.c. }\right\}+\left[\Phi^{\dagger} e^{2 g V} \Phi\right]_{D}=\ldots+F^{\dagger} F+\frac{1}{2} D^{2}+g A A^{\dagger} D .
$$


Solving the Lagrange field equations for $F$ and $D$,

$$
\begin{array}{lll}
\frac{\partial \mathscr{L}}{\partial F}=0 & \Longleftrightarrow & F=0, \\
\frac{\partial \mathscr{L}}{\partial D}=0 & \Longleftrightarrow & D=-g A^{\dagger} A .
\end{array}
$$

Inserting these results back into eq. (6.36) [where the terms not explicitly given can be found in eqs. (6.10) and (6.35)] yields the Lagrangian in terms of its physical fields,

$$
\begin{aligned}
\mathscr{L}= & -\frac{1}{4} F_{\mu \nu} F^{\mu \nu}+i \lambda^{\dagger} \bar{\sigma}^{\mu} \partial_{\mu} \lambda+\left(\mathcal{D}_{\mu} A\right)\left(\mathcal{D}^{\mu} A\right)^{\dagger}+i \psi^{\dagger} \bar{\sigma}^{\mu} \mathcal{D}_{\mu} \psi \\
& +i \sqrt{2} g\left(A^{\dagger} \lambda \psi-A \lambda^{\dagger} \psi^{\dagger}\right)-\frac{1}{2} g^{2}\left(A^{\dagger} A\right)^{2} .
\end{aligned}
$$

Thus, a potential for the scalar field $A$ has been generated,

$$
V_{\text {scalar }}=\frac{1}{2} g^{2}\left(A^{\dagger} A\right)^{2} .
$$

There is one more possible term, called the Fayet-Iliopoulos term [121], that can appear in a renormalizable SUSY U(1) gauge theory Lagrangian,

$$
\mathscr{L}_{\mathrm{FI}}=2 \xi[V]_{D}=\xi D+\text { total divergence. }
$$

This modifies the form of $D$ obtained in eq. (6.38),

$$
D=-g A^{\dagger} A-\xi,
$$

which in turn modifies the scalar potential,

$$
V_{\text {scalar }}=\frac{1}{2}\left[g A^{\dagger} A+\xi\right]^{2} .
$$

The existence of a quartic scalar coupling proportional to the square of the gauge coupling (in the presence or absence of a Fayet-Iliopoulos term) is another manifestation of SUSY.

\subsection{Generalizing to more than one chiral superfield}

With only one chiral superfield, it was not possible to include a superpotential $W(\Phi)$ in our gauge theory, since $W$ is a holomorphic function of a charged field and hence not gauge-invariant. But, a theory with more than one charged chiral superfield can admit a gauge invariant superpotential.

For example, consider a set of charged chiral superfields $\Phi_{i}$ with U(1) charges $q_{i}$, which transform under $\mathrm{U}(1)$ as

$$
\Phi_{i} \rightarrow e^{-2 i g q_{i} \Lambda} \Phi_{i} .
$$


Suppose that a gauge-invariant superpotential can be constructed, $W\left(\Phi_{i}\right)$. When we solve for the auxiliary field $F_{i}$, we will obtain

$$
F_{i}=-\left(\frac{d W}{d A_{i}}\right)^{\dagger},
$$

as before [cf. eq. (4.132)], which provides the $F$-term contributions to the scalar potential,

$$
V_{\text {scalar }} \ni \sum_{i}\left|\frac{d W}{d A_{i}}\right|^{2} .
$$

When we solve for the auxiliary field $D$, we obtain a contribution from each scalar $A_{i}$,

$$
D=-\xi-\sum_{i} q_{i} g A_{i}^{\dagger} A_{i} .
$$

The corresponding $D$-term contributions to the scalar potential are

$$
V_{\text {scalar }} \ni \frac{1}{2}\left[\xi+\sum_{i} g q_{i} A^{\dagger} A\right]^{2} .
$$

Including both the $F$-term and $D$-term contributions yields the following scalar potential,

$$
V_{\text {scalar }}=\sum_{i}\left|\frac{d W}{d A_{i}}\right|^{2}+\frac{1}{2}\left[\xi+\sum_{i} g q_{i} A^{\dagger} A\right]^{2},
$$

which can also be conveniently written as

$$
V_{\text {scalar }}=\sum_{i} F_{i}^{\dagger} F_{i}+\frac{1}{2} D^{2},
$$

where $F$ and $D$ are given by eqs. (6.45) and (6.48), respectively. Note that the form of the scalar potential [either eq. (6.49) or (6.50)] makes clear that $V_{\text {scalar }} \geq 0$. This observation will play an important role in the theory of supersymmetry breaking, which is treated in Section 7 .

The above results can now be used to construct the supersymmetric extension of QED. The superfield content of SUSY-QED consists of a real vector superfield $V$, a chiral superfield $\Phi_{+}$with charge $q=1$, and a chiral superfield $\Phi_{-}$with charge $q=-1$. The unique renormalizable, gaugeinvariant superpotential is

$$
W\left(\Phi_{+}, \Phi_{-}\right)=m \Phi_{+} \Phi_{-} .
$$

The $R$-charges of both $\Phi_{+}$and $\Phi_{-}$can be chosen to be +1 , in which case the theory is also $R$-invariant. The construction of the SUSY-QED Lagrangian is left as an exercise (see Problem 32). 


\subsection{SUSY Yang-Mills theory coupled to supermatter}

The construction of the supersymmetric generalization of Yang-Mills theory, i.e., a non-abelian gauge theory coupled to matter, is more complicated than the case of an abelian gauge theory treated in previous sections. In this subsection, we will summarize the main modifications. The reader can fill in the details with the help of Refs. [10,33].

Consider a non-abelian compact simple Lie group G, with generators $T^{a}$ that satisfy commutation relations,

$$
\left[T^{a}, T^{b}\right]=i f_{a b c} T^{c} .
$$

It is convenient to normalize the generators of the defining (fundamental) representation of $\mathrm{G}$ such that,

$$
\operatorname{Tr}\left(T^{a} T^{b}\right)=\frac{1}{2} \delta_{a b} .
$$

The vector superfield, $V^{a}$, possesses an adjoint index $a$, which runs over the generators of G. Thus, we can define the matrix gauge superfield,

$$
V \equiv V^{a} T^{a} .
$$

The gauge transformation law for $V$ given in eq. (6.16) is significantly more complicated in the case of a non-abelian gauge theory,

$$
e^{2 g V} \longrightarrow e^{-2 i g \Lambda^{\dagger}} e^{2 g V} e^{2 i g \Lambda},
$$

where $\Lambda \equiv\left(\Lambda^{a} T^{a}\right)_{i j}$ is the matrix chiral superfield gauge transformation parameter.

The chiral superfields are now multiplets corresponding to representation $R$ of the gauge group $\mathrm{G}$, transforming as ${ }^{39}$

$$
\Phi_{i} \rightarrow\left(e^{-2 i g \Lambda}\right)_{i j} \Phi_{j},
$$

which provides the generalization of eq. (6.32) to a nonabelian gauge group. Note that $\Phi_{i}^{\dagger}\left(e^{2 g V}\right)_{i j} \Phi_{j}$ is gauge-invariant, if the gauge transformation law for $V$ is given by eq. (6.55).

Likewise, we define a matrix version of the nonabelian field-strength superfield, $\mathcal{W}_{\alpha} \equiv \mathcal{W}_{\alpha}^{a} T^{a}$, where

$$
\mathcal{W}_{\alpha}=-\frac{1}{8 g} \bar{D}^{2} e^{-2 g V} D_{\alpha} e^{2 g V} .
$$

Unlike the abelian case, $\mathcal{W}_{\alpha}$ is not gauge-invariant. However it transforms as an adjoint field,

$$
\mathcal{W}_{\alpha} \rightarrow e^{-2 i g \Lambda} \mathcal{W}_{\alpha} e^{2 i g \Lambda},
$$

\footnotetext{
${ }^{39}$ When acting on the $\Phi_{i}$, one employs the generators $T^{a}$ in the representation $R$.
} 
so that $\operatorname{Tr}\left(\mathcal{W}^{\alpha} \mathcal{W}_{\alpha}\right)$ is gauge-invariant. In the $\mathrm{WZ}$ gauge, ${ }^{40}$ when expanded in component fields, $\mathcal{W}_{\alpha}^{a}$ depends only on the physical fields, $\lambda^{a}, F^{\mu \nu a}$ and the auxiliary field $D^{a}$,

$$
\mathcal{W}_{\alpha}^{a}=-i \lambda_{\alpha}^{a}+\theta_{\alpha} D^{a}-\frac{1}{2} i\left(\sigma^{\mu} \bar{\sigma}^{\nu} \theta\right)_{\alpha} F_{\mu \nu}^{a}-\sigma^{\mu}\left(\mathscr{D}_{\mu a b} \lambda^{\dagger b}\right)_{\alpha} \theta \theta,
$$

where

$$
\mathscr{D}_{\mu a b} \equiv \delta_{a b} \partial_{\mu}+g f_{a b c} V_{\mu}^{c},
$$

is the gauge-covariant derivative in the adjoint representation, and

$$
F_{\mu \nu}^{a}=\partial_{\mu} V_{\nu}^{a}-\partial_{\nu} V_{\mu}^{a}-g f_{a b c} V_{\mu}^{b} V_{\nu}^{c}
$$

is the nonabelian field strength tensor.

\subsection{The SUSY Lagrangian}

The Lagrangian for SUSY Yang-Mills theory coupled to supermatter is given by

$\mathscr{L}=\left[\frac{1}{2} \int d^{2} \theta \operatorname{Tr}\left(\mathcal{W}^{\alpha} \mathcal{W}_{\alpha}\right)+\right.$ h.c. $]+\int d^{4} \theta \Phi^{\dagger} e^{2 g V} \Phi+\left[\int d^{2} \theta W\left(\Phi_{k}\right)+\right.$ h.c. $]$

In contrast to the abelian gauge theory, no Fayet-Iliopoloulos term is allowed since $\left[D^{a}\right]_{D}$ carries an adjoint index and thus is not gauge invariant. The superpotential $W\left(\Phi_{k}\right)$ is assumed to be a gauge-invariant holomorphic function of the chiral superfields. The chiral superfields $\Phi_{k}$ taken together transform under a reducible $d$-dimensional representation $R=\oplus_{k} R_{k}$ of the gauge group $\mathrm{G}$, where $d=\sum_{k} \operatorname{dim} R_{k}$. In terms of component fields, eq. (6.62) yields

$$
\begin{aligned}
\mathscr{L}= & -\frac{1}{4} F_{\mu \nu}^{a} F^{\mu \nu a}+i \lambda^{\dagger a} \bar{\sigma}^{\mu}\left(\mathscr{D}_{\mu} \lambda\right)^{a}+\frac{1}{2} D^{a} D^{a}+F_{i}^{\dagger} F_{i}+\left(\mathcal{D}_{\mu} A\right)_{i}\left(\mathcal{D}^{\mu} A\right)_{i}^{\dagger} \\
& +i \psi_{i}^{\dagger} \bar{\sigma}^{\mu}\left(\mathcal{D}_{\mu} \psi\right)_{i}+g A_{i}^{\dagger} T_{i j}^{a} A_{j} D^{a}+i g \sqrt{2}\left(A_{i}^{\dagger} T_{i j}^{a} \psi_{j} \lambda^{a}-\lambda^{\dagger a} \psi_{i}^{\dagger} T_{i j}^{a} A_{j}\right) \\
& +F_{i} \frac{d W}{d A_{i}}+F_{i}^{\dagger}\left(\frac{d W}{d A_{i}}\right)^{\dagger}-\frac{1}{2} \frac{d^{2} W}{d A_{i} d A_{j}} \psi_{i} \psi_{j}-\frac{1}{2}\left(\frac{d^{2} W}{d A_{i} d A_{j}}\right)^{\dagger} \psi_{i}^{\dagger} \psi_{j}^{\dagger}
\end{aligned}
$$

where there is an implicit sum over repeated indices, and the labels $i$ and $j$ run over $1,2, \ldots, d$. The corresponding covariant derivative, when acting on

\footnotetext{
${ }^{40}$ In contrast to the abelian case, the expansion of $\mathcal{W}_{\alpha}^{a}$ in terms of its component fields in the nonabelian case will necessarily contain gauge artifacts. After imposing the WZ gauge condition, the expansion of $\mathcal{W}_{\alpha}^{a}$ in terms of its component fields resembles the corresponding expression of SUSY abelian gauge theory [cf. eq. (6.5)].
} 
the component fields $A_{i}$ and $\psi_{i}$, is $\mathcal{D}_{\mu}=\mathbb{1} \partial_{\mu}+i g T^{a} V_{\mu}^{a}$, where $\mathbb{1}$ is the $d \times d$ identity matrix and the generators $T^{a}$ are in the reducible representation $R$ of the group $\mathrm{G}$.

Note that the interactions of the matter fermions and the gauginos with the gauge fields are dictated by gauge invariance (via the gauge covariant derivative) and do not depend on supersymmetry. In contrast, the Yukawa interaction of the gaugino with the matter fermion and its scalar partner (with a coupling proportional to the gauge coupling $g$ ) is a consequence of supersymmetry, and relates the gauge and Yukawa couplings that otherwise would be independent.

We can now eliminate the auxiliary fields $F_{i}$ and $D^{a}$ by employing the Lagrange field equations. We end up with

$$
F_{i}=-\left(\frac{d W}{d A_{i}}\right)^{\dagger}, \quad D^{a}=-g A_{i}^{\dagger} T_{i j}^{a} A_{j} .
$$

Substituting back into eq. (6.63) yields the following scalar potential,

$$
V_{\text {scalar }}=\sum_{i}\left|\frac{d W}{d A_{i}}\right|^{2}+\frac{1}{2} g^{2}\left(A_{i}^{\dagger} T_{i j}^{a} A_{j}\right)^{2} .
$$

Equivalently, we can write:

$$
V_{\text {scalar }}=\frac{1}{2} D^{a} D^{a}+\sum_{i} F_{i}^{\dagger} F_{i} .
$$

Eqs. (6.65) and (6.66) provide the nonabelian generalization of eqs. (6.49) and (6.50). As in the abelian case, $V_{\text {scalar }} \geq 0$.

If we drop the requirement of renormalizability, then we can generalize the action of a SUSY-Yang Mills theory coupled to supermatter,

$$
\begin{aligned}
\mathscr{L}= & \frac{1}{2} \int d^{4} \theta\left[K\left(e^{2 g V} \Phi, \Phi^{\dagger}\right)+K\left(\Phi, \Phi^{\dagger} e^{2 g V}\right)\right]+\left[\int d^{2} \theta W\left(\Phi_{i}\right)+\text { h.c. }\right] \\
& +\left[\frac{1}{4} \int d^{2} \theta f_{a b}(\Phi) \mathcal{W}^{\alpha a} \mathcal{W}_{\alpha}^{b}+\text { h.c. }\right],
\end{aligned}
$$

where $K$ is the Kähler potential and $f_{a b}(\Phi)$ is a holomorphic function of the chiral superfields called the gauge kinetic function. In renormalizable global supersymmetry, the minimal versions of the Kähler potential and gauge kinetic function are used:

$$
\begin{aligned}
K\left(e^{2 g V} \Phi, \Phi^{\dagger}\right) & =K\left(\Phi, \Phi^{\dagger} e^{2 g V}\right)=\Phi^{\dagger} e^{2 g V} \Phi, \\
f_{a b}(\Phi) & =\delta_{a b} .
\end{aligned}
$$

The generalization of the SUSY Lagrangian to a theory based on a gauge group that is a direct product of compact simple Lie groups and $\mathrm{U}(1)$ factors is straightforward. There is a gauge field strength tensor and a separate gauge coupling constant corresponding to each group in the direct product. Details are left for the reader. 


\subsection{Problems}

Problem 30. Show that $\mathcal{W}_{\alpha}$ is invariant under the gauge transformation of eq. (6.16).

Problem 31. Show that the kinetic energy term given by eq. (6.34) is invariant under the gauge transformations for $\Phi$ and $\Phi^{\dagger}$ given in eq. (6.32) and $V \rightarrow V+i\left(\Lambda-\Lambda^{\dagger}\right)$.

Problem 32. Construct the full SUSY QED Lagrangian in the WessZumino gauge. Show that the physical states of the theory consist of a Dirac fermion (the "electron"), two complex scalar "selectrons," usually denoted by $\widetilde{e}_{L}$ and $\widetilde{e}_{R}$, a massless photon, and a massless photino. Check that the number of bosonic and fermionic degrees of freedom are equal, both off-shell and on-shell.

Problem 33. Consider the SUSY QED theory examined in Problem 32. However, this time do not impose the Wess-Zumino gauge condition. Instead, explore the consequences of adding the following supersymmetric gauge fixing term [122-124],

$$
\mathscr{L}_{\mathrm{GF}}=-\frac{1}{8 \alpha}\left[\left(D^{2} V\right)\left(\bar{D}^{2} V\right)\right]_{D},
$$

where $\alpha$ is the gauge fixing parameter.

Problem 34. Starting from the case where the gauge group $G$ is nonabelian, show that the gauge transformation law for the gauge superfield $V$, as deduced from eq. (6.55), reduces to $V \rightarrow V+i\left(\Lambda-\Lambda^{\dagger}\right)$ in the abelian limit. Likewise, show that $\mathcal{W}_{\alpha}$ as given in eq. (6.57) reduces to $\mathcal{W}_{\alpha}=-\frac{1}{4} \bar{D}^{2} D_{\alpha} V$ in the abelian limit.

Problem 35. Evaluate the contribution of the Kähler potential terms to the Lagrangian given in eq. (6.67) in terms of the component fields. Show that your result reduces to eq. (5.86) in the limit of $g \rightarrow 0$.

Problem 36. Evaluate the contribution of the gauge kinetic function terms to the Lagrangian given in eq. (6.67) in terms of the component fields. How does your result simplify in the abelian limit?

Problem 37. Starting from eq. (6.67), solve for the auxiliary fields $F_{i}$ and $D^{a}$ using the Lagrange field equations. Using these results, determine the form of the scalar potential that generalizes the results of eqs. (6.65) and (6.66). 


\section{Supersymmetry Breaking}

If supersymmetry were an exact symmetry of nature, then particles and their superpartners, which differ in spin by half a unit, would be degenerate in mass. Since superpartners have not (yet) been observed, supersymmetry must be a broken symmetry. In light of the non-observation of supersymmetric particles at the LHC, the energy scale of supersymmetry breaking must lie above $1 \mathrm{TeV}$.

The fundamental mechanism responsible for supersymmetry breaking is presently unknown. In Section 7.1, we describe some general considerations related to SUSY breaking, and we examine several possible frameworks for the spontaneous breaking of SUSY. In Section 7.2, we examine constraints on mass splittings within supermultiplets in the presence of SUSY-breaking. The possible origins of SUSY-breaking dynamics is surveyed in Section 7.3. Finally, in Section 7.4, we examine a more agnostic approach, in which the supersymmetry of the effective low energy theory at the $\mathrm{TeV}$ scale is softly broken. In such an approach, we identify the possible soft-supersymmetry breaking terms that can appear in the Lagrangian, without making assumptions about their fundamental origin.

\subsection{Spontaneous SUSY breaking}

In Section 4.2, we derived eq. (4.28), which states that the energy operator $P^{0}$ for a supersymmetric theory is given by

$$
P^{0}=\frac{1}{2 t}\left(Q_{1} Q_{1}^{\dagger}+Q_{1}^{\dagger} Q_{1}+Q_{2} Q_{2}^{\dagger}+Q_{2}^{\dagger} Q_{2}\right),
$$

where $t$ is real and positive (conventionally, $t=2$ ). Since the right-hand side of eq. (7.1) is positive semi-definite, it follows that the vacuum energy is zero if and only if the vacuum is supersymmetric:

$$
\left\langle 0\left|P^{0}\right| 0\right\rangle=0 \quad \Longleftrightarrow \quad Q_{\alpha}|0\rangle=0 .
$$

Moreover, assuming the absence of fermion condensation, ${ }^{41}$ the vacuum energy can be identified as the vacuum expectation value of the scalar potential. That is, in the case of a supersymmetric vacuum,

$$
\left\langle 0\left|P^{0}\right| 0\right\rangle=0 \quad \Longleftrightarrow \quad\left\langle 0\left|V_{\text {scalar }}\right| 0\right\rangle=0 .
$$

\footnotetext{
${ }^{41}$ That is, we assume the absence of a fermion bilinear covariant, with the properties of a Lorentz scalar, that acquires a nonzero vacuum expectation value.
} 
To appreciate the significance of $\left\langle 0\left|V_{\text {scalar }}\right| 0\right\rangle=0$, recall eq. (6.66), which we repeat below for the convenience of the reader,

$$
V_{\text {scalar }}=\frac{1}{2} D^{a} D^{a}+\sum_{i} F_{i}^{*} F_{i} .
$$

It follows that if the vacuum is supersymmetric, then the vacuum expectation values of the auxiliary fields must vanish,

$$
\left\langle 0\left|F_{i}\right| 0\right\rangle=\left\langle 0\left|D^{a}\right| 0\right\rangle=0 .
$$

One can reach the same conclusion by considering the transformation laws of the field components of a superfield. For a chiral superfield, the component fermion field transforms according to,

$$
\delta_{\xi} \psi_{\alpha i}=i\left[\xi Q+\xi^{\dagger} Q^{\dagger}, \psi_{\alpha i}\right]=-i \sqrt{2}\left(\sigma^{\mu} \xi^{\dagger}\right)_{\alpha} \partial_{\mu} A_{i}+\sqrt{2} \xi_{\alpha} F_{i} .
$$

By Lorentz invariance, $\left\langle 0\left|\partial_{\mu} A_{i}\right| 0\right\rangle=0$. Hence,

$$
\left\langle 0\left|\left[\xi Q+\xi^{\dagger} Q^{\dagger}, \psi_{\alpha i}\right]\right| 0\right\rangle=\sqrt{2} \xi_{\alpha}\left\langle 0\left|F_{i}\right| 0\right\rangle .
$$

Thus, if $Q_{\alpha}|0\rangle=0$ and $Q_{\dot{\alpha}}^{\dagger}|0\rangle=0$, then $\left\langle 0\left|F_{i}\right| 0\right\rangle=0$. Likewise, for a real vector superfield, the component gaugino field transforms according to,

$$
\delta_{\xi} \lambda_{\alpha}^{a}=i\left[\xi Q+\xi^{\dagger} Q^{\dagger}, \lambda_{\alpha}^{a}\right]=i \xi_{\alpha} D^{a}+\frac{1}{2}\left(\sigma^{\mu} \bar{\sigma}^{\nu}\right)_{\alpha}{ }^{\beta} \xi_{\beta} F_{\mu \nu}^{a} .
$$

Since $\left\langle 0\left|F_{\mu \nu}^{a}\right| 0\right\rangle=0$ (again, by Lorentz invariance), it follows that

$$
\left\langle 0\left|\left[\xi Q+\xi^{\dagger} Q^{\dagger}, \lambda_{\alpha}^{a}\right]\right| 0\right\rangle=i \xi_{\alpha}\left\langle 0\left|D^{a}\right| 0\right\rangle .
$$

Thus, if $Q_{\alpha}|0\rangle=0$ and $Q_{\dot{\alpha}}^{\dagger}|0\rangle=0$, then $\left\langle 0\left|D^{a}\right| 0\right\rangle=0$.

If at least one of the components of the auxiliary fields $F_{i}$ or $D_{a}$ has a nonzero vacuum expectation value, then SUSY is spontaneously broken. Mechanisms of spontaneous SUSY breaking fall into two possible categories: $F$-type breaking, if $\left\langle 0\left|F_{i}\right| 0\right\rangle \neq 0$ for some $i$, and $D$-type breaking if $\left\langle 0\left|D^{a}\right| 0\right\rangle \neq 0$ for some $a$.

\subsubsection{The O'Raifeartaigh mechanism (F-type breaking)}

One way to spontaneously break SUSY is to construct a model in which it is impossible to simultaneously solve the Lagrange field equations for all the components of the auxiliary fields, $F_{i}$. This is the O'Raifeartaigh mechanism [125], ${ }^{42}$ where the SUSY breaking arises entirely from a nonzero $F$-term vacuum expectation value. ${ }^{43}$

\footnotetext{
${ }^{42} \mathrm{~A}$ well-known supersymmetric joke: a graduate student returns to the University for the fall semester after spending a month at TASI earlier in the summer. The professor says to the student, "Welcome back! I see that one of the lecture courses you attended at TASI was an introduction to supersymmetry. So, did you learn anything useful from these lectures?" The student replies, "I learned how to spell O'Raifeartaigh's name." ${ }^{43}$ Implicitly, we are assuming here that if the $D$-term is present, then $\left\langle D^{a}\right\rangle=0$.
} 
Consider the set of equations,

$$
F_{i}^{\dagger}=-\frac{d W}{d A_{i}}=0 .
$$

A solution to these equations corresponds to the existence of a choice of the scalar fields, $A_{i}$, such that all the equations, $F_{i}^{\dagger}=0$, are fulfilled. Suppose that a solution, $A_{i}=v_{i}$, solves these equations. In light of eq. (7.4), this solution must correspond to a minimum of the scalar potential, which we identify as the vacuum (ground) state of the theory. Since $F_{i}^{\dagger}=0$ implies that $F_{i}=0$, we can conclude that $\left\langle 0\left|F_{i}\right| 0\right\rangle=0$ (for all $i$ ). If no solution to eq. (7.10) exists, then it must be true that $\left\langle 0\left|F_{i}\right| 0\right\rangle \neq 0$ for some $i$. In this latter case, SUSY must be spontaneously broken.

The simplest O'Raifeartaigh model that exhibits $F$-term SUSY breaking contains three chiral superfields and is treated in Problem 38.

\subsubsection{D-type breaking via the Fayet-Iliopoulos term}

Consider SUSY-QED with a superpotential given by eq. (6.51) and a FayetIliopoulos term. Using eqs. (6.45) and (6.47), the resulting scalar potential [eq. (6.50)] is given by

$$
V_{\text {scalar }}=\left|F_{+}\right|^{2}+\left|F_{-}\right|^{2}+\frac{1}{2} D^{2},
$$

where

$$
F_{ \pm}=-m A_{ \pm}, \quad D=-g\left(\left|A_{+}\right|^{2}-\left|A_{-}\right|^{2}\right)-\xi .
$$

Suppose that $m^{2}>g \xi$. One can check that the minimum of the scalar potential occurs for $\left\langle A_{+}\right\rangle=\left\langle A_{-}\right\rangle=0$. Moreover, at the scalar potential minimum, $\left\langle F_{+}\right\rangle=\left\langle F_{-}\right\rangle=0$, whereas $\langle D\rangle=-\xi \neq 0$. Thus, in this model SUSY breaking arises entirely from a nonzero $D$-term vacuum expectation value. Additional aspects of this model are treated in Problems 40 and 41.

\subsubsection{The goldstino}

From Goldstone's theorem, we know that the spontaneous breaking of a continuous symmetry (with bosonic generators) gives rise to a massless boson called the Nambu-Goldstone boson. Analogously, the spontaneous breaking of supersymmetry, whose algebra contains fermionic generators, gives rise to a massless fermion called the Goldstone fermion, which is more commonly known as the goldstino [126].

Theorem 5. If SUSY is spontaneously broken, then there exists a massless spin-1/2 fermion in the spectrum called the goldstino. 
Proof. Although this theorem can be proven rigorously, independently of perturbation theory, it is instructive to exhibit a proof based on a tree-level analysis of a SUSY nonabelian gauge theory coupled to supermatter. The scalar potential is given by eq. (7.4) where [cf. eq. (6.64)],

$$
F_{i}=-\left(\frac{d W}{d A_{i}}\right)^{\dagger}, \quad D^{a}=-g A_{i}^{\dagger} T_{i j}^{a} A_{j} .
$$

At the scalar potential minimum, where $\partial V / \partial A_{j}=0$, the scalar fields are equal to their vacuum expectation values, $A_{j}=\left\langle A_{j}\right\rangle$. Then,

$$
0=\left(\frac{\partial V}{\partial A_{j}}\right)_{\langle A\rangle}=-\left.g A_{i}^{\dagger} T_{i j}^{a} D^{a}\right|_{\langle A\rangle}-\left.\sum_{i} \frac{\partial^{2} W}{\partial A_{i} \partial A_{j}} F_{i}\right|_{\langle A\rangle} .
$$

Hence,

$$
\sum_{i}\left\langle\frac{\partial^{2} W}{\partial A_{i} \partial A_{j}}\right\rangle\left\langle F_{i}\right\rangle=-g\left\langle A_{i}\right\rangle^{\dagger} T_{i j}^{a}\left\langle D^{a}\right\rangle .
$$

The superpotential $W$ must be a gauge invariant function of the chiral superfields. That is,

$$
W(\Phi)=W\left(e^{-2 i g \Lambda} \Phi\right) .
$$

where $\Lambda \equiv \Lambda^{a} T^{a}$ is the matrix chiral superfield gauge transformation parameter. Taking $\Lambda^{a}$ infinitesimal and expanding to first order yields

$$
\frac{d W}{d \Phi_{i}} T_{i j}^{a} \Phi_{j}=0 .
$$

Evaluating the hermitian conjugate of this expression, setting $\theta=\theta^{\dagger}=0$, and taking the vacuum expectation value of the resulting equation, we end up with

$$
\left\langle F_{i}\right\rangle T_{j i}^{a}\left\langle A_{j}\right\rangle^{\dagger}=0 .
$$

The fermion masses can be determined from the SUSY Lagrangian given by eq. (6.63) after setting the scalar fields to their vacuum expectation values,

$$
\begin{aligned}
-\mathscr{L}_{\text {mass }} & =\frac{1}{2}\left\langle\frac{\partial^{2} W}{\partial A_{i} \partial A_{j}}\right\rangle \psi_{i} \psi_{j}-i \sqrt{2} g\left\langle A_{i}\right\rangle^{\dagger} T_{i j}^{a} \psi_{j} \lambda^{a}+\text { h.c. } \\
& =\frac{1}{2}\left(\begin{array}{ll}
\psi_{i} & -i \lambda^{b}
\end{array}\right)\left(\begin{array}{cc}
\left\langle\frac{\partial^{2} W}{\partial A_{i} \partial A_{j}}\right\rangle & \sqrt{2} g\left\langle A_{j}\right\rangle^{\dagger} T_{j i}^{a} \\
\sqrt{2} g\left\langle A_{i}\right\rangle^{\dagger} T_{i j}^{b} & 0
\end{array}\right)\left(\begin{array}{c}
\psi_{j} \\
\\
-i \lambda^{b}
\end{array}\right) .
\end{aligned}
$$


Using eqs. (7.15) and (7.18), one can verify that the fermion mass matrix given in eq. (7.20) possesses a zero eigenvalue,

$$
\left(\begin{array}{cc}
\left\langle\frac{\partial^{2} W}{\partial A_{i} \partial A_{j}}\right\rangle & \sqrt{2} g\left\langle A_{j}\right\rangle^{\dagger} T_{j i}^{a} \\
\sqrt{2} g\left\langle A_{i}\right\rangle^{\dagger} T_{i j}^{b} & 0
\end{array}\right)\left(\begin{array}{c}
\left\langle F_{j}\right\rangle \\
\\
\frac{1}{\sqrt{2}}\left\langle D^{a}\right\rangle
\end{array}\right)=0,
$$

under the assumption that at least one of the auxiliary field vacuum expectation values is nonzero. The corresponding eigenvector, $\left(\left\langle F_{j}\right\rangle, \frac{1}{\sqrt{2}}\left\langle D^{a}\right\rangle\right)$, can be identified with the massless goldstino, $\widetilde{G}$. That is,

$$
\widetilde{G}=\left\langle F_{j}\right\rangle \psi_{j}-\frac{i}{\sqrt{2}}\left\langle D^{a}\right\rangle \lambda^{a} .
$$

The existence of the goldstino in the fermion mass spectrum is a consequence of the assumption that the vacuum is not invariant under SUSY transformations, in which case at least one of the auxiliary field vacuum expectation values is nonzero, as assumed below eq. (7.21). In contrast, if the vacuum is supersymmetric, then $\left\langle F_{j}\right\rangle=\left\langle D^{a}\right\rangle=0$, in which case eqs. (7.15) and (7.18) are trivially satisfied. Hence in this case, one cannot conclude that a zero eigenvalue of the fermion mass matrix exists.

\subsection{Mass Sum rules}

If SUSY is broken, then there is no expectation that particles in a would-be supermultiplet are degenerate in mass. If the SUSY breaking is spontaneous, then there is still some memory of supersymmetry in the properties of the SUSY-broken theory. In particular, the mass spectrum of the spontaneously broken SUSY theory satisfies certain sum rules that reflect the fact the spontaneous breaking of the supersymmetry is inherently soft [127].

To exhibit such sum rules, we return to the Lagrangian of the SUSY nonabelian gauge theory coupled to supermatter given in eq. (6.63). We set the scalar fields and the auxiliary fields to their vacuum expectation values and compute the resulting tree-level mass spectrum.

The spin-1 masses arise from

$$
\mathscr{L}_{\text {mass }}=\left(\mathcal{D}_{\mu} A\right)\left(\mathcal{D}^{\mu} A\right)^{\dagger},
$$

where $\mathcal{D}_{\mu}=\partial_{\mu}+i g T^{a} V_{\mu}^{a}$. It is convenient to write the gauge boson squaredmass matrix as follows,

$$
\left(M_{1}^{2}\right)_{a b}=2 g^{2}\left\langle A_{i}^{\dagger}\right\rangle T_{i j}^{a} T_{j k}^{b}\left\langle A_{k}\right\rangle=2\left\langle\frac{\partial D^{a}}{\partial A_{k}^{\dagger}} \frac{\partial D^{b}}{\partial A_{k}}\right\rangle,
$$


where we have made use of $D^{a}=-g A_{i}^{\dagger} T_{i j}^{a} A_{j}$ [cf. eq. (6.64)]. Likewise, we can rewrite the spin-1/2 mass matrix [previously obtained in eq. (7.20)] as,

$$
M_{\frac{1}{2}}=\left(\begin{array}{cc}
\left\langle-\frac{\partial F_{i}^{\dagger}}{\partial A_{j}}\right\rangle & -\sqrt{2}\left\langle\frac{\partial D^{a}}{\partial A_{i}}\right\rangle \\
-\sqrt{2}\left\langle\frac{\partial D^{b}}{\partial A_{j}}\right\rangle & 0
\end{array}\right) .
$$

The spin-0 masses arise from the scalar potential, $V \equiv V_{\text {scalar }}$. Identifying the terms quadratic in the scalar field,

$$
-\mathscr{L}_{\text {mass }}=\frac{1}{2}\left(\begin{array}{ll}
A_{i} & A_{j}^{\dagger}
\end{array}\right)\left(\begin{array}{ll}
\left\langle\frac{\partial^{2} V}{\partial A_{i} \partial A_{k}^{\dagger}}\right\rangle & \left\langle\frac{\partial^{2} V}{\partial A_{i} \partial A_{\ell}}\right\rangle \\
\left\langle\frac{\partial^{2} V}{\partial A_{j}^{\dagger} \partial A_{k}^{\dagger}}\right\rangle & \left\langle\frac{\partial^{2} V}{\partial A_{j}^{\dagger} \partial A_{\ell}}\right\rangle
\end{array}\right)\left(\begin{array}{c}
A_{k}^{\dagger} \\
A_{\ell}
\end{array}\right) .
$$

The scalar squared-mass matrix given above will be denoted by $M_{0}^{2}$.

The elements of the scalar squared-mass matrix can be rewritten in terms of derivatives of the auxiliary fields $F_{i}$ and $D^{a}$. For example, noting that eq. (7.13) implies that $F$ is a function of $A^{\dagger}$ (and likewise, $F^{\dagger}$ is a function of $A$ ), then it follows from eq. (7.4) that

$$
\frac{\partial^{2} V}{\partial A_{i} \partial A_{k}^{\dagger}}=\frac{\partial F_{m}^{\dagger}}{\partial A_{i}} \frac{\partial F_{m}}{\partial A_{k}^{\dagger}}+\frac{\partial D^{a}}{\partial A_{k}^{\dagger}} \frac{\partial D^{a}}{\partial A_{i}}+D^{a} \frac{\partial^{2} D^{a}}{\partial A_{k}^{\dagger} \partial A_{i}} .
$$

One can now evaluate the trace of the various squared-mass matrices,

$$
\begin{aligned}
\operatorname{Tr} M_{1}^{2} & =2\left\langle\frac{\partial D^{a}}{\partial A_{k}^{\dagger}} \frac{\partial D^{a}}{\partial A_{k}}\right\rangle \\
\operatorname{Tr} M_{\frac{1}{2}}^{\dagger} M_{\frac{1}{2}} & =\left\langle\frac{\partial F_{i}}{\partial A_{k}^{\dagger}} \frac{\partial F_{i}^{\dagger}}{\partial A_{k}^{\dagger}}\right\rangle+4\left\langle\frac{\partial D^{a}}{\partial A_{k}^{\dagger}} \frac{\partial D^{a}}{\partial A_{k}}\right\rangle, \\
\operatorname{Tr} M_{0}^{2} & =2\left\langle\frac{\partial F_{i}^{\dagger}}{\partial A_{k}} \frac{\partial F_{i}}{\partial A_{k}^{\dagger}}\right\rangle+2\left\langle\frac{\partial D^{a}}{\partial A_{k}^{\dagger}} \frac{\partial D^{a}}{\partial A_{k}}\right\rangle+2\left\langle D^{a} \frac{\partial^{2} D^{a}}{\partial A_{k}^{\dagger} \partial A_{k}}\right\rangle,
\end{aligned}
$$

where there are implicit sums over each pair of repeated indices. We can simplify the last term of eq. (7.30) using $D^{a}=-g A_{i}^{\dagger} T_{i j}^{a} A_{j}$ to obtain.

$$
\operatorname{Tr} M_{0}^{2}=2\left\langle\frac{\partial F_{i}}{\partial A_{k}^{\dagger}} \frac{\partial F_{i}^{\dagger}}{\partial A_{k}}\right\rangle+2\left\langle\frac{\partial D^{a}}{\partial A_{k}^{\dagger}} \frac{\partial D^{a}}{\partial A_{k}}\right\rangle-2 g\left\langle D^{a}\right\rangle \operatorname{Tr} T^{a} .
$$


It then follows that

$$
\operatorname{Tr}\left(M_{0}^{2}-2 M_{\frac{1}{2}}+3 M_{1}^{2}\right)=-2 g\left\langle D^{a}\right\rangle \operatorname{Tr} T^{a} .
$$

We recognize the left-hand side of eq. (7.32) as a supertrace, which is defined as the following weighted sum of traces,

$$
\operatorname{Str} M^{2} \equiv \sum_{J}(-1)^{2 J}(2 J+1) \operatorname{Tr} M_{J}^{2}
$$

where $M_{J}^{2}$ is the squared-mass matrix of real spin- $J$ fields. ${ }^{44}$ Note the $(-1)^{2 J}$ factor, so that bosons contribute positively and fermions negatively to the sum over $J$. As applied to a SUSY nonabelian gauge theory coupled to supermatter, the sum is taken over $J=0, \frac{1}{2}$ and 1 . Hence, eq. (7.32) assumes the following simple form,

$$
\operatorname{Str} M^{2}=-2 g\left\langle D^{a}\right\rangle \operatorname{Tr} T^{a} .
$$

The mass sum rule can provide a useful check on the phenomenological viability of theories with tree-level spontaneous supersymmetry breaking. Let us now see how this applies in several cases.

\subsection{The origin of SUSY-breaking dynamics}

\subsubsection{Models of tree-level spontaneous SUSY breaking}

In the case of $F$-type breaking (i.e., the O'Raifeartaigh model), in which $\left\langle F_{i}\right\rangle \neq 0$ and $\left\langle D^{a}\right\rangle=0$, eq. (7.34) yields

$$
\text { Str } M^{2}=0 \text {. }
$$

For example, consider the matter sector of SUSY-QED, which contains two chiral supermultiplets [cf. eq. (6.51)]. The corresponding spectrum contains a four-component Dirac electron and its two complex scalar superpartners, the selectrons (denoted by $\widetilde{e}_{1}$ and $\widetilde{e}_{2}$ ). If SUSY is spontaneously broken by an $F$-term vacuum expectation value, then eq. (7.35) yields

$$
m_{\tilde{e}_{1}}^{2}+m_{\tilde{e}_{2}}^{2}=2 m_{e}^{2},
$$

so that one selectron would be heavier than the electron and the other selectron would be lighter than the electron. Clearly, this is very bad for phenomenology, since experiment demands that all superpartner masses must be significantly heavier than their SM counterparts.

Consider next $D$-type breaking with $\left\langle F_{i}\right\rangle=0$ and $\left\langle D^{a}\right\rangle \neq 0$ in a nonabelian gauge theory. In this case, $\operatorname{Tr} T^{a}=0$ and we again conclude that

\footnotetext{
${ }^{44}$ Note that complex fields are equivalent to two mass-degenerate real fields.
} 
$\operatorname{Str} M^{2}=0$. However, it turns out that when the scalar potential is minimized, it is always possible to find a vacuum in which $\left\langle D^{a}\right\rangle=0$. Hence, $D$-term SUSY-breaking is not possible in this case (see Problem 43).

Finally, consider $D$-type breaking in a gauge theory with a U(1) factor. The Standard Model provides an example of this case. But in the Standard Model, the hypercharge generator satisfies $\operatorname{Tr} Y=0$ when summed over one generation of matter. Hence we again find that $\operatorname{Str} M^{2}=0$. It is possible to construct models of $D$-type SUSY breaking via the Fayet-Iliopoulos term $\xi$. In such models, $\langle D\rangle$ is proportional to $\xi$, as shown below eq. (7.12). However, no realistic models of this type are known.

Based on the above considerations, we conclude that the mass sum rule severely constrains tree-level SUSY-breaking models. Indeed, no phenomenologically realistic tree-level spontaneously broken SUSY model has ever been successfully constructed.

\subsubsection{Gauge-mediated SUSY breaking}

One way to avoid the tyranny of the mass sum rule is to consider models in which the radiative corrections to the tree-level masses are significant. In general, there is no reason why the radiative corrections should respect the tree-level relations derived in Section 7.2. For example, one can construct models with two distinct sectors of supermatter, which are coupled by the exchange of gauge bosons. The particles of the Standard Model (SM) reside in one of the supermatter sectors, whereas the source of SUSY-breaking (SSB) is located in the second supermatter sector, whose characteristic mass scale, $M_{\mathrm{SSB}}$, is assumed to be significantly above $1 \mathrm{TeV}$. Indeed, in this second supermatter sector, the masses of particles and their superpartners are split due to SUSY-breaking, while respecting the tree-level mass sum rule obtained in eq. (7.34). In this case, tree-level SUSY-breaking is phenomenologically viable in light of the large characteristic mass scale $M_{\mathrm{SSB}}$ that governs the SSB sector.

In such a setup, SUSY is unbroken in the SM sector at tree level, in which case $\operatorname{Str} M^{2}=0$ is trivially satisfied (see Problem 42). However, there exist radiative corrections to the sum rule induced by loops involving the supermatter of the SSB sector. These corrections are responsible for SUSYbreaking in the SM sector and the corresponding mass splitting between the SM particles and their superpartners. Moreover, these mass splittings are totally radiative in nature and not subject to the tree-level sum rule of eq. (7.34). Models can easily be constructed in which the masses of the SM 
superpartners are all raised above $1 \mathrm{TeV}$, thereby avoiding conflict with the current LHC searches. The end result is SUSY-breaking in the SM that is phenomenologically viable.

In the scenario outlined above, SUSY-breaking is communicated to the SM-sector via a messenger mechanism, in which the messengers consists of gauge bosons that couple both to the SM sector and the SSB sector. Models of this type provide examples of gauge-mediated SUSY breaking (GMSB). Details of GMSB model building lie beyond the scope of these lectures. For further details, you may consult Refs. [39, 44, 46].

\subsubsection{Local supersymmetry and the super-Higgs mechanism}

Another way of evading the tyranny of the mass sum rule is to consider models with local supersymmetry.

In these lectures, we have focused on theories with global supersymmetry, where the anticommuting SUSY translation parameter $\xi$ is independent of the position $x$. Suppose we attempt to generalize this to local supersymmetry, where $\xi=\xi(x)$. Since the spinorial SUSY generators satisfy $\left\{Q_{\alpha}, \bar{Q}_{\dot{\beta}}\right\}=2 \sigma_{\alpha \dot{\beta}}^{\mu} P_{\mu}$, a theory of local supersymmetry must also be invariant under local spacetime translations, in which the translation depends on the position. A theory that possesses a local spacetime translation symmetry is a theory of gravity! Hence, a locally supersymmetric theory is a theory of gravity plus supersymmetry, i.e., supergravity [24, 28].

We have already encountered the massless supermultiplet that contains the spin-3/2 gravitino and the spin- 2 graviton. Suppose we couple this supermultiplet to ordinary supermatter. In addition, suppose that the local supersymmetry is broken, which will generate a mass splitting within the graviton supermultiplet. We require that the graviton remain massless, while the gravitino acquires mass. This can be accomplished via the super Higgs mechanism [128, 129].

We have seen in Section 7.1.3 that in models of spontaneously-broken global supersymmetry, the spectrum includes a massless goldstino. In models of spontaneously-broken supergravity, the goldstino is "absorbed" by the gravitino via the super-Higgs mechanism. Initially, a massless gravitino possesses only two helicity states, $\lambda= \pm \frac{3}{2}$. In the super-Higgs mechanism, the goldstino provides $\lambda= \pm \frac{1}{2}$ helicity states for a massive gravitino. That is, the goldstino is removed from the physical spectrum and the gravitino acquires a mass (denoted by $m_{3 / 2}$ ). The gravitino now possesses the four helicity states, $\lambda= \pm \frac{3}{2}, \pm \frac{1}{2}$, as expected for a massive spin- $\frac{3}{2}$ particle. 
In spontaneously broken supergravity, the tree-level mass sum rule obtained in eq. (7.34) is modified. For example, if $N$ chiral supermultiplets are minimally coupled to supergravity, then [130],

$$
\operatorname{Str} M^{2}=(N-1)\left(2 m_{3 / 2}^{2}-\kappa\left\langle D^{a} D^{a}\right\rangle\right)-2 g\left\langle D^{a}\right\rangle T^{a},
$$

where $\kappa=\left(8 \pi G_{N}\right)^{1 / 2}=(8 \pi)^{1 / 2} M_{\mathrm{PL}}^{-1}$. Typical models of interest have $\left\langle D^{a}\right\rangle=0$, in which case [131],

$$
\operatorname{Str} M^{2}=2(N-1) m_{3 / 2}^{2} .
$$

If $m_{3 / 2} \gtrsim \mathcal{O}(1 \mathrm{TeV})$, then one expects the superpartner masses of SM particles to lie in the $\mathrm{TeV}$ regime.

\subsubsection{Gravity-mediated SUSY-breaking}

Consider again the framework of two distinct sectors of supermatter that are initially uncoupled. We identify one of the sectors as the SM sector where the SM particles and their superpartners reside. In the second socalled "hidden" sector, SUSY is spontaneously broken.

Supergravity models provide a natural mechanism for transmitting the SUSY breaking of the hidden sector to the particle spectrum of the SM sector. In models of gravity-mediated SUSY breaking, gravity is the messenger of supersymmetry breaking [31, 132]. More precisely, SUSY breaking in the SM sector is mediated by effects of gravitational strength (suppressed by inverse powers of the Planck mass). The induced mass splittings between the SM particles and their superpartners are of $\mathcal{O}\left(m_{3 / 2}\right)$, whereas the gravitino couplings are roughly gravitational in strength.

Under certain theoretical assumptions on the structure of the Kähler potential (the so-called sequestered form introduced in Ref. [133]), SUSY breaking is due entirely to the super-conformal (super-Weyl) anomaly, which is common to all supergravity models. This approach is called anomaly-mediated supersymmetry breaking (AMSB). Indeed, anomaly mediation is more generic than originally conceived, and provides a ubiquitous source of supersymmetry breaking [134, 135].

\subsection{A phenomenological approach: soft SUSY-breaking}

If SUSY-breaking arises due to gauge-mediated SUSY-breaking or gravitymediated SUSY-breaking, then we can formally integrate out the SSB sector physics at the mass scale $M_{\mathrm{SSB}}$ that characterizes the fundamental SUSYbreaking dynamics. For example, in the case of gravity-mediated SUSY 
breaking, we identify $M_{\mathrm{SSB}}=M_{\mathrm{PL}}$. In GMSB models, $M_{\mathrm{SSB}}$ can be much smaller than $M_{\mathrm{PL}}$ but still significantly larger than the scale of electroweak symmetry breaking.

The end result is an effective broken supersymmetric theory whose Lagrangian consists of supersymmetric terms and explicit SUSY-breaking terms. The explicit SUSY-breaking terms that are present in the effective low-energy theory (which is valid at energy scales below $M_{\mathrm{SSB}}$ ) are "soft." The meaning of soft in this context will be explained shortly.

The phenomenological approach to SUSY-breaking takes the point of view that the fundamental dynamics of SUSY-breaking is unknown. Therefore, we should simply parameterize SUSY breaking in the low-energy effective theory by including all possible soft-SUSY-breaking terms. The coefficients of these terms will be taken to be arbitrary (to be determined by experiment). Ultimately, these parameters will provide clues to the structure of the fundamental dynamics that is responsible for SUSY-breaking.

\subsubsection{A catalog of soft-SUSY-breaking terms}

The most general set of soft-SUSY-breaking terms in a super-Yang Mills theory coupled to supermatter was first elucidated by Girardello and Grisaru in Ref. [136],

$$
-\mathscr{L}_{\text {soft }}=m_{i j}^{2} A_{i}^{\dagger} A_{j}+\frac{1}{2}\left[m_{a b} \lambda^{a} \lambda^{b}+\text { h.c. }\right]+[w(A)+\text { h.c. }],
$$

where there is an implicit sum over repeated indices. The scalar squaredmass matrix $m_{i j}^{2}$ is hermitian and the gaugino mass matrix $m_{a b}$ is complex symmetric. The function $w(A)$ is a holomorphic cubic multinomial of the scalar fields,

$$
w(A)=c_{i} A_{i}+b_{i j} A_{i} A_{j}+a_{i j k} A_{i} A_{j} A_{k} .
$$

Note that $c_{i}=0$ in the absence of any gauge singlet fields. In the literature, the $b_{i j}$ are called the $B$-terms and the $a_{i j k}$ are called the $A$-terms. Note the corresponding mass dimensions, $\left[b_{i j}\right]=2$ and $\left[a_{i j k}\right]=1$.

Dimension-4 terms are not included in eq. (7.39), since nonsupersymmetric dimension- 4 terms would constitute a hard breaking of supersymmetry [137]. One interesting feature of eq. (7.39) is the absence of non-supersymmetric fermion mass terms, $m_{i j} \psi_{i} \psi_{j}+$ h.c., and nonholomorphic cubic terms in the scalar fields (e.g., $A_{i} A_{j} A_{k}^{\dagger}$, etc.). Although such terms are technically soft in models with no gauge singlets [138-142], theses terms rarely arise in actual models of fundamental SUSY-breaking, or if present are highly suppressed [137]. Henceforth, we shall neglect them. 
In general, there is no relation between $w(A)$ and the superpotential, which under the assumption of renormalizability has the following generic form,

$$
W(\Phi)=\kappa_{i} \Phi_{i}+\mu_{i j} \Phi_{i} \Phi_{j}+\lambda_{i j k} \Phi_{i} \Phi_{j} \Phi_{k} .
$$

But, some models of fundamental SUSY breaking yield the relations,

$$
c_{i}=C \kappa_{i}, \quad b_{i j}=B \mu_{i j}, \quad a_{i j k}=A \lambda_{i j k},
$$

which relate the coefficients of $w(A)$ to the coefficients of $W(\Phi)$.

\subsubsection{Soft vs. hard SUSY breaking and the reappearance of quadratic divergences}

Consider the one-loop effective potential for a gauge theory coupled to matter,

$$
V_{\text {eff }}(A)=V_{\text {scalar }}(A)+V^{(1)}(A) .
$$

If we regulate the divergence of the one-loop correction by a momentum cutoff $\Lambda$, then [143]

$$
V^{(1)}(A)=\frac{\Lambda^{2}}{32 \pi^{2}} \operatorname{Str} M_{i}^{2}(A)+\frac{1}{64 \pi^{2}} \operatorname{Str}\left\{M_{i}^{4}(A)\left[\ln \left(\frac{M_{i}^{2}(A)}{\Lambda^{2}}\right)-\frac{1}{2}\right]\right\}
$$

where $M_{i}^{2}(A)$ are the relevant squared-mass matrices for spin $0, \frac{1}{2}$ and 1 , in which the scalar vacuum expectation values are replaced by the corresponding scalar fields, $A$.

Eq. (7.44) implies that both in supersymmetric theories and in the case of spontaneously broken SUSY (assuming in the latter that all U(1) generators are traceless), we have $\operatorname{Str} M^{2}=0$, in which case the quadratic divergences [i.e., the terms proportional to $\Lambda^{2}$ in eq. (7.44)] cancel exactly! In Ref. [136], Girardello and Grisaru showed that if explicit SUSY breaking terms are present, then there is a catalog of possible explicit SUSY-breaking terms for which $\operatorname{Str} M_{i}^{2}(A)$ is a constant independent of the scalar fields, $A$. Such terms shift the vacuum energy, but in the context of quantum field theory they have no observable effect. Terms with such properties are deemed "soft," and are given in eq. (7.39). ${ }^{45}$ In contrast, hard SUSYbreaking terms will generate quadratically divergent terms in $V^{(1)}$ that are scalar-field-dependent. This is a signal that some of the parameters of the low-energy effective theory are quadratically sensitive to UV physics.

\footnotetext{
${ }^{45}$ Non-holomorphic cubic terms and mass terms of fermions that reside in a chiral supermultiplet can generate quadratically divergent terms in $V^{(1)}$ that are linear in the scalar fields, $A$. However, if no gauge singlet fields exist in the model, then terms that are linear in $A$ are absent due to gauge invariance.
} 


\subsubsection{Soft SUSY-breaking: an effective theory perspective}

Consider a set of light chiral superfields $\Phi$ and a set of heavy chiral superfields $\Omega$ associated with a mass scale $M \equiv M_{\mathrm{SSB}}$. Furthermore, assume that SUSY-breaking is generated by an $F$-term that resides in the SSB sector,

$$
\left\langle F_{\Omega}\right\rangle=f \neq 0 .
$$

One can integrate out the physics of the SSB sector, as shown in the following examples [136, 144, 145].

Example 8. Consider a holomorphic cubic multinomial of chiral superfields $\Phi$, which we denote by $\widetilde{w}(\Phi)$. A possible term in the effective Lagrangian is

$$
\frac{1}{M} \int d^{2} \theta \Omega \widetilde{w}(\Phi),
$$

since $\Omega \widetilde{w}(\Phi)$ is a term in the superpotential. The factor of $M^{-1}$ appears on the basis of dimensional analysis. In particular, note the mass dimensions, $[\widetilde{w}]=3,[\Omega]=1$ and $\left[\int d^{2} \theta\right]=1$.

Since the vacuum expectation value of $F_{\Omega}$, denoted by $\left\langle F_{\Omega}\right\rangle=f$, is nonzero, it follows that $\langle\Omega\rangle \ni \theta \theta f$. Inserting this into eq. (7.46) yields,

$$
\frac{1}{M} \int d^{2} \theta \theta \theta f \widetilde{w}(\Phi)=\frac{f}{M} \widetilde{w}(A),
$$

which produces the term, $w(A)=(f / M) \widetilde{w}(A)$, in our catalog of $\delta \mathscr{L}_{\text {soft }}$ given in eq. (7.39).

In order to achieve soft-SUSY-breaking masses in the low-energy effective theory of order $1 \mathrm{TeV}$, one must require that $f / M \sim \mathcal{O}(1 \mathrm{TeV})$. For example, in gravity-mediated SUSY breaking, $M \sim M_{\mathrm{PL}}$, in which case $f \sim\left(10^{11} \mathrm{GeV}\right)^{2}$. Note that $f^{1 / 2}$ identifies the energy scale of the fundamental SUSY breaking.

Example 9. Another possible term in the effective Lagrangian is

$$
\frac{1}{M^{2}} \int d^{4} \theta \Phi_{i}^{\dagger}\left(e^{2 g V}\right)_{i j} \Phi_{j} \Omega^{\dagger} \Omega
$$

which would contribute to the Kähler potential. Setting $\langle\Omega\rangle=\theta \theta f$ and evaluating the result in the Wess-Zumino gauge,

$$
\frac{f^{2}}{M^{2}} \int d^{4} \theta(\theta \theta)\left(\theta^{\dagger} \theta^{\dagger}\right) \Phi_{i}^{\dagger}\left(e^{2 g V}\right)_{i j} \Phi_{j}=\frac{f^{2}}{M^{2}} A^{\dagger} A .
$$

Thus, the low-energy effective theory contains a scalar squared-mass term of order $f / M$, which we again recognize as one of the soft-SUSY-breaking terms of eq. (7.39). 
Example 10. Finally, one additional possible term in the effective Lagrangian is

$$
\frac{1}{M} \int d^{2} \theta \Omega \operatorname{Tr}\left(W^{\alpha} W_{\alpha}\right),
$$

which would contribute to the gauge kinetic function. Setting $\langle\Omega\rangle=\theta \theta f$,

$$
\frac{f}{M} \int d^{2} \theta \theta \theta \operatorname{Tr}\left(W^{\alpha} W_{\alpha}\right)=-\frac{f}{M} \operatorname{Tr}\left(\lambda^{\alpha} \lambda_{\alpha}\right),
$$

which yields a gaugino mass term of order $f / M$.

We have thus demonstrated how the possible soft-SUSY-breaking terms of eq. (7.39) can arise in the low-energy effective theory after integrating out the physics associated with the SSB sector.

\subsection{Problems}

Problem 38. An O'Raifeartaigh model that exhibits F-term SUSY breaking must involve at least three chiral superfields [125]. One of the simplest models of this type has the following superpotential,

$$
W\left(\Phi_{1}, \Phi_{2}, \Phi_{3}\right)=\lambda \Phi_{1}\left(\Phi_{3}^{2}-m^{2}\right)+\mu \Phi_{2} \Phi_{3},
$$

where $\lambda$ is dimensionless and $\mu$ and $m$ are mass parameters. Evaluate the corresponding F-terms, $F_{1}, F_{2}$ and $F_{3}$ and write out the scalar potential,

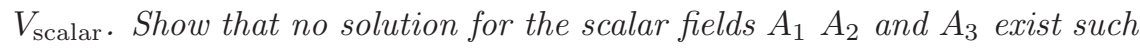
that $F_{1}=F_{2}=F_{3}=0$. Conclude that SUSY is spontaneously broken.

Problem 39. Find the minimum of $V_{\text {scalar }}$ obtained in Problem 38, and verify that $\left\langle 0\left|V_{\text {scalar }}\right| 0\right\rangle>0$. Identify the goldstino of this model. Finally, compute the mass spectrum of the fermions and bosons and verify that the mass sum rule, eq. (7.35), is satisfied.

Problem 40. Show that in the case of SUSY-QED with a Fayet-Iliopoulos term and $m^{2}>g \xi$ [cf. eqs. (7.11) and (7.12)], SUSY is broken and the goldstino can be identified as the photino (the supersymmetric partner of the photon). In the case of $m^{2}<g \xi$, is SUSY broken? Is the U(1) gauge symmetry broken?

Problem 41. Referring back to Problem 40, determine the masses of the electron and its scalar partners and the masses of the photon and photino in the two cases of $m^{2}>g \xi$ and $m^{2}<g \xi$, respectively. Evaluate $\operatorname{Str} M^{2}$ in both cases, and compare with eq. (7.34).

Problem 42. Show that the sum rule of eq. (7.34) is valid in the limit of exact SUSY, i.e., when the masses of bosons and fermions are equal. 
Problem 43. Show that in a SUSY nonabelian gauge theory that is coupled to supermatter, only F-type SUSY breaking is allowed. To prove this statement, assume that a solution to $\left\langle F_{i}\right\rangle=0$ exists and show that one can always find a choice of scalar fields $A_{i}$ that provide a solution to eq. (7.10) such that $\left\langle D^{a}\right\rangle=0$ for all $a$.

HINT: If the $A_{i}$ provide a solution to eq. (7.10), then so do the corresponding gauge transformed scalar fields, $\left(e^{-2 i g \Lambda}\right)_{i j} A_{j}$. The key observation is that the superpotential is a holomorphic function of the scalar fields $A_{i}$. Hence, one can generate additional solutions to eq. (7.10) by taking g complex, which will modify $\left\langle D^{a}\right\rangle$. Conclude that there must then be a set of $A_{i}$ such that $\left\langle F_{i}\right\rangle=\left\langle D^{a}\right\rangle=0$. See Ref. [2] for further details.

\section{Supersymmetric extension of the Standard Model (MSSM)}

With the necessary SUSY technology now in hand, we are ready to study its realization in extensions to the SM. In this section, we describe the minimal supersymmetric extension of the Standard Model (MSSM). Much of the presentation of this section follows Ref. [48], where many of the relevant references to the original literature can be found.

In Section 8.1, we begin by presenting the MSSM field content. We then specify the $\mathrm{SU}(3) \times \mathrm{SU}(2) \times \mathrm{U}(1)$ gauge-invariant superpotential for the chiral superfields in Section 8.2. Given the superfield formalism developed in Sections 5 and 6 , all the supersymmetric interactions of the theory are now determined. At this stage, the supersymmetry is still an exact symmetry.

We introduce SUSY breaking in the MSSM in Section 8.3. Since the fundamental origin of SUSY-breaking is unknown, we parametrize the SUSYbreaking by adding all possible soft-SUSY-breaking terms consistent with the $\mathrm{SU}(3) \times \mathrm{SU}(2) \times \mathrm{U}(1)$ gauge symmetry and a discrete $B-L$ symmetry. In Section 8.4, we count the number of parameters that govern the MSSM. The resulting MSSM particle spectrum and Higgs boson spectrum are exhibited in Sections 8.5 and 8.6, respectively. Finally, in Section 8.7, we demonstrate the unification of gauge couplings in the MSSM.

As in the SM, the neutrinos of the MSSM are massless. To incorporate massive neutrinos, one can introduce right-handed neutrinos and employ the seesaw mechanism. It is then a simple matter to extend the MSSM by adding a SM singlet superfield that contains a right-handed neutrino and the corresponding sneutrino superpartner. We shall not present this construction in these lectures; for further details, see e.g. Ref. [146]. 


\subsection{Field content of the MSSM}

\subsubsection{MSSM superfields and their component fields}

The minimal supersymmetric extension of the Standard Model (MSSM) contains the fields of the two-Higgs-doublet extension of the SM and their corresponding superpartners. The gauge fields and their superpartners are contained in real vector supermultiplets. These gauge supermultiplets consist of the $\mathrm{SU}(3) \times \mathrm{SU}(2) \times \mathrm{U}(1)$ gauge bosons and their gaugino fermionic superpartners. The matter fields and their superpartners reside in chiral supermultiplets. The three generations of quark and lepton supermultiplets consist of left-handed quarks and leptons and their scalar superpartners (squarks and sleptons), and the corresponding antiparticles. The Higgs supermultiplets consist of two complex Higgs doublets, their higgsino fermionic superpartners, and the corresponding antiparticles. The MSSM fields and their gauge quantum numbers are shown in Table 5.

Table 5.: The fields of the MSSM and their $\mathrm{SU}(3) \times \mathrm{SU}(2) \times \mathrm{U}(1)$ quantum numbers are listed. The electric charge is given in terms of the third component of the weak isospin $T_{3}$ and $\mathrm{U}(1)$ hypercharge $Y$ by $Q=T_{3}+\frac{1}{2} Y$. For simplicity, only one generation of quarks and leptons is exhibited. The left-handed chargeconjugated quark and lepton fields are denoted by a superscript $c$. In particular, $f_{L}^{c} \equiv P_{L} f^{c}=P_{L} C \bar{f}^{\top}=C \bar{f}_{R}^{\top}$, following the notation of Ref. [147], where $f$ is a four-component fermion field. The $L$ and $R$ subscripts of the squark and slepton fields indicate the chirality of the corresponding fermionic superpartners.

\begin{tabular}{|c|c|c|c|c|c|c|}
\hline \multicolumn{7}{|c|}{ Field content of the MSSM } \\
\hline $\begin{array}{c}\text { Super- } \\
\text { multiplets }\end{array}$ & $\begin{array}{c}\text { Super- } \\
\text { field }\end{array}$ & $\begin{array}{c}\text { Bosonic } \\
\text { fields }\end{array}$ & $\begin{array}{c}\text { Fermionic } \\
\text { partners }\end{array}$ & $\mathrm{SU}(3)$ & $\mathrm{SU}(2)$ & $\mathrm{U}(1)$ \\
\hline gluon/gluino & $\widehat{V}_{8}$ & $g$ & $\widetilde{g}$ & 8 & 1 & 0 \\
gauge boson/ & $\widehat{V}$ & $W^{ \pm}, W^{0}$ & $\widetilde{W}^{ \pm}, \widetilde{W}^{0}$ & 1 & 3 & 0 \\
gaugino & $\widehat{V}^{\prime}$ & $B$ & $\widetilde{B}^{-}$ & 1 & 1 & 0 \\
\hline slepton/ & $\widehat{L}^{c}$ & $\left(\widetilde{\nu}_{L}, \widetilde{e}_{L}^{-}\right)$ & $\left(\nu, e^{-}\right)_{L}$ & 1 & 2 & -1 \\
lepton & $\widehat{E}^{c}$ & $\widetilde{e}_{R}^{+}$ & $e_{L}^{c}$ & 1 & 1 & 2 \\
\hline squark/ & $\widehat{Q}^{c}$ & $\left(\widetilde{u}_{L}, \widetilde{d}_{L}\right)$ & $(u, d)_{L}$ & 3 & 2 & $1 / 3$ \\
quark & $\widehat{U}^{c}$ & $\widetilde{u}_{R}^{*}$ & $u_{L}^{c}$ & $\overline{3}$ & 1 & $-4 / 3$ \\
& $\widehat{D}^{c}$ & $\widetilde{d}_{R}^{*}$ & $d_{L}^{c}$ & $\overline{3}$ & 1 & $2 / 3$ \\
\hline Higgs boson/ & $\widehat{H}_{d}$ & $\left(H_{d}^{0}, H_{d}^{-}\right)$ & $\left(\widetilde{H}_{d}^{0}, \widetilde{H}_{d}^{-}\right)$ & 1 & 2 & -1 \\
higgsino & $\widehat{H}_{u}$ & $\left(H_{u}^{+}, H_{u}^{0}\right)$ & $\left(\widetilde{H}_{u}^{+}, \widetilde{H}_{u}^{0}\right)$ & 1 & 2 & 1 \\
\hline
\end{tabular}


Table 5 shows that one Higgs doublet superfield has hypercharge -1 , and the other has hypercharge +1 . The distinction between hypercharge \pm 1 is irrelevant in a non-supersymmetric quantum field theory, where complex scalar fields are always accompanied by their hermitian conjugates. However, in supersymmetric models the distinction is important, because the corresponding Higgs superfields are used to construct the superpotential. Since the superpotential must be holomorphic, i.e. depend only on chiral superfields and not their hermitian conjugates, it is important to keep track of the quantum numbers of the chiral superfields of the model.

\subsubsection{Anomaly cancellation and the second Higgs doublet}

The enlarged Higgs sector of the MSSM constitutes the minimal structure needed to guarantee the cancellation of gauge anomalies generated by the higgsino superpartners that can appear as internal lines in one-loop triangle diagrams with three external electroweak gauge bosons.

Potentially problematic anomalies arise from one-loop $V V A$ and $A A A$ triangle diagrams with three external gauge bosons, and fermions running around the loop [where $V$ refers to a $\gamma_{\mu}$ (vector) vertex and $A$ refers to a $\gamma_{\mu} \gamma_{5}$ (axial vector) vertex]. An anomalous theory violates unitarity and fails as a consistent quantum field theory. Thus, we need to make sure all gauge anomalies cancel when summed over all triangle diagrams with fixed external gauge fields [148].

The anomalies will cancel if certain group theoretical constraints are satisfied. In particular, the trace of the product of the relevant generators appearing at the external vertices must vanish,

$$
\begin{array}{lll}
W^{i} W^{j} B \text { triangle } & \Longleftrightarrow & \operatorname{Tr}\left(T_{3}^{2} Y\right)=0, \\
B B B \text { triangle } & \Longleftrightarrow & \operatorname{Tr}\left(Y^{3}\right)=0 .
\end{array}
$$

In the Standard Model, the fermion contributions to $\operatorname{Tr}\left(Y^{3}\right)$ sum to zero:

$$
\operatorname{Tr}\left(Y^{3}\right)_{\mathrm{SM}}=3\left(\frac{1}{27}+\frac{1}{27}-\frac{64}{27}+\frac{8}{27}\right)-1-1+8=0 .
$$

In contrast, in the MSSM, if we only add the higgsinos $\left(\widetilde{H}_{u}^{+}, \widetilde{H}_{u}^{0}\right)$, the resulting anomaly factor is $\operatorname{Tr}\left(Y^{3}\right)=\operatorname{Tr}\left(Y^{3}\right)_{\mathrm{SM}}+2$, leading to a gauge anomaly. To cancel this, we must add a second higgsino doublet with opposite hypercharge, $\left(\widetilde{H}_{d}^{0}, \widetilde{H}_{d}^{-}\right)$.

There is an independent argument for requiring the second Higgs doublet in the MSSM. With only one Higgs doublet, one cannot generate mass for both "up"-type and "down"-type quarks (and charged leptons) in a way that is consistent with a holomorphic superpotential. 


\subsubsection{Suppressed baryon and lepton number violation}

It is an experimental fact that baryon number $B$ and lepton number $L$ are, to a very good approximation, global symmetries of nature. If neutrinos are Majorana fermions, then $L$-violation is present but strongly suppressed, with neutrino masses of order $v^{2} / M$, where $v$ is the scale of electroweak symmetry breaking and $M \gg v$. No $B$-violation has yet been experimentally observed. Moreover, the current bounds on the proton lifetime suggest that the mass scale associated with baryon number violation cannot be below about $10^{16} \mathrm{GeV}$, which is a characteristic scale of grand unification.

One of the remarkable features of the SM is that the suppression of $B$ and $L$-violating processes is a natural feature of the model. That is, the SM Lagrangian possesses an accidental global $B-L$ symmetry due to the fact that all renormalizable terms of the Lagrangian (with dimension four or less) that can be composed of SM fields preserve the $B$ and $L$ global symmetries. Indeed, $B$ and $L$-violating operators composed of SM fields must have dimension $d=5$ or larger [149-151].

For example, consider the dimension-five $L$-violating operator,

$$
\mathscr{L}_{5}=-\frac{f_{m n}}{M}\left(\epsilon^{i j} L_{i}^{m} H_{j}\right)\left(\epsilon^{k \ell} L_{k}^{n} H_{\ell}\right)+\text { h.c. },
$$

where $f$ is a coefficient that depends on the lepton generation (labeled by $m$ and $n), H_{j}$ is the complex Higgs doublet field and $L_{i}^{a} \equiv\left(\nu_{L}^{a}, \ell_{L}^{a}\right)$ is the doublet of two-component lepton fields. After electroweak symmetry breaking, the neutral component of the doublet Higgs field acquires a vacuum expectation value, and a Majorana mass matrix for the neutrinos is generated. The dimension-five term given by eq. (8.2) is generated by new physics beyond the SM at the scale $M$. Likewise, one can construct dimension-six $B$-violating operators composed of SM fields that allow, e.g., for proton decay, which is suppressed by $v^{2} / M_{\mathrm{G}}^{2}$. Such terms can be generated, e.g., in grand unified theories with a characteristic mass scale $M_{\mathrm{G}}$. In general, $B$ and $L$-violating effects are suppressed by $(v / M)^{d-4}$, where $M$ is the characteristic mass scale of the physics that generates the corresponding higher dimensional operator (of dimension $d$ ).

Unfortunately, the suppression of $B$ and $L$-violation is not guaranteed in a generic supersymmetric extension of the Standard Model. For example, it is possible to construct gauge invariant supersymmetric dimension-four $B$ and $L$-violating operators made up of fields of SM particles and their superpartners. Such operators, if present in the theory, would yield a proton decay rate many orders of magnitude larger than the current experimental 
bound. To avoid this catastrophic prediction, one can introduce an additional symmetry in the supersymmetric theory that will eliminate the $B$ and $L$-violating operators of dimension $d \leq 4$. Further details are provided in the next subsection. Nevertheless, one must admit that the SM provides a more satisfying explanation for approximate $B$ and $L$ conservation than does its supersymmetric extension.

\subsection{The superpotential of the MSSM}

Given the chiral and gauge superfield content of the MSSM, we must now specify the superpotential. The most general $\mathrm{SU}(3) \times \mathrm{SU}(2) \times \mathrm{U}(1)$ gaugeinvariant superpotential (omitting the right-handed neutrino superfield) is

$$
\begin{aligned}
W= & \left(h_{u}\right)_{m n} \widehat{Q}_{m} \cdot \widehat{H}_{u} \widehat{U}_{n}^{c}+\left(h_{d}\right)_{m n} \widehat{H}_{d} \cdot \widehat{Q}_{m} \widehat{D}_{n}^{c} \\
& +\left(h_{e}\right)_{m n} \widehat{H}_{d} \cdot \widehat{L}_{m} \widehat{E}_{n}^{c}+\mu \widehat{H}_{u} \cdot \widehat{H}_{d}+W_{\mathrm{RPV}},
\end{aligned}
$$

where $m$ and $n$ label the generations. That is, $h_{u}, h_{d}$ and $h_{e}$ are $3 \times 3$ matrix Yukawa couplings. Note that color indices have been suppressed, and we employ a dot product notation for the singlet combination of two $\mathrm{SU}(2)$ doublets. For example,

$$
\widehat{H}_{u} \cdot \widehat{H}_{d} \equiv \epsilon^{i j} \widehat{H}_{u i} \widehat{H}_{d j}=\widehat{H}_{u}^{+} \widehat{H}_{d}^{-}-\widehat{H}_{u}^{0} \widehat{H}_{d}^{0} .
$$

The so-called $\mu$-term above is the supersymmetric analog of the Higgs boson squared-mass term of the SM.

In addition to the supersymmetric generalization of the SM Yukawa couplings and the $\mu$-term, the gauge symmetries of the superpotential also allow for a number of new terms that violate $B-L$ conservation. As discussed in Section 8.1.3, this is in contrast to the SM where there are no $B$ or $L$-violating interactions at the renormalizable level. The $B-L$ violating terms of the supersymmetric model arise due to the presence of $W_{\mathrm{RPV}}$ in eq. (8.3) and are given by,

$$
\begin{aligned}
W_{\mathrm{RPV}}= & \left(\lambda_{L}\right)_{p m n} \widehat{L}_{p} \widehat{L}_{m} \widehat{E}_{n}^{c}+\left(\lambda_{L}^{\prime}\right)_{p m n} \widehat{L}_{p} \widehat{Q}_{m} \widehat{D}_{n}^{c} \\
& +\left(\lambda_{B}\right)_{p m n} \widehat{U}_{p}^{c} \widehat{D}_{m}^{c} \widehat{D}_{n}^{c}+\left(\mu_{L}\right)_{p} \widehat{H}_{u} \widehat{L}_{p} .
\end{aligned}
$$

Note that the term proportional to $\lambda_{B}$ violates $B$, while the other three terms violate $L$. The $L$-violating term proportional to $\mu_{L}$ is the generalization of the $\mu \widehat{H}_{u} \widehat{H}_{d}$ term, in which the $Y=-1$ Higgs supermultiplet $\widehat{H}_{d}$ is replaced by the lepton supermultiplet $\widehat{L}_{p}$. Indeed, if $L$ violation is present, then there is no distinction between $\widehat{L}$ and $\widehat{H}_{d}$, since the gauge quantum numbers of these two superfields are identical. 
If all terms in $W_{\mathrm{RPV}}$ were allowed, the resulting model would predict a proton decay rate many orders of magnitude larger than the current experimental bound. This can be avoided by imposing an appropriate discrete symmetry that would eliminate the undesirable terms in $W$.

The standard choice in constructing the MSSM is to set $W_{R P V}=0$. There are a number of ways to accomplish this. First, one one could directly impose a $B-L$ symmetry. Alternatively, one can set $W_{R P V}=0$ by introducing a matter parity, under which $\widehat{Q}, \widehat{U}^{c}, \widehat{D}^{c}, \widehat{L}$ and $\widehat{E}^{c}$ are odd, and $\widehat{H}_{u}$ and $\widehat{H}_{d}$ are even. Finally, a third option is to impose an $R$-invariant superpotential. As discussed in Section 5.6, $W$ is $R$-invariant if the $R$ charges of the chiral superfields are chosen such that $R(W)=2$. Thus, if we choose $R$ charges of $+\frac{1}{2}$ for $\widehat{Q}, \widehat{U}^{c}, \widehat{D}^{c}, \widehat{L}, \widehat{E}^{c}$ and $R$ charges of +1 for $\widehat{H}_{u}, \widehat{H}_{d}$, then the condition of $R$-invariance sets $W_{\mathrm{RPV}}=0$.

One has to make sure that whichever symmetry one chooses to set $W_{\mathrm{RPV}}=0$ is also consistent with the soft-SUSY-breaking terms that are subsequently added to the model. In particular, in the case of the $R$ invariance, recall that $R(\lambda)=1$, which forbids the gaugino mass term,

$$
m_{\lambda}\left(\lambda \lambda+\lambda^{\dagger} \lambda^{\dagger}\right)
$$

But phenomenology requires massive gauginos. This motivates the use of $R$-parity, described in the following subsection, rather than $R$-invariance.

\subsubsection{R-parity}

The gaugino mass term in eq. (8.6) is an allowed soft-SUSY-breaking term. If this term is added to a theory with an $R$-invariant superpotential, then the continuous $\mathrm{U}(1)_{R}$ symmetry is broken down to a discrete $\mathbb{Z}_{2}$ symmetry, called $R$-parity $[152,153]$. One can check that the $R$-parity of a particle with baryon number $B$, lepton number $L$ and spin $S$ is given by

$$
R=(-1)^{3(B-L)+2 S} .
$$

It is sufficient to impose $R$-parity invariance in order to set $W_{\mathrm{RPV}}=0,{ }^{46}$ which is equivalent to imposing the $B-L$ discrete symmetry. For the remainder of these lectures, we shall assume that $R$-parity is conserved.

One can use eq. (8.7) to deduce the $R$-parity quantum numbers of all SM particles and their supersymmetric partners,

$$
R= \begin{cases}+1, & \text { for all SM particle particles } \\ -1, & \text { for all superpartners }\end{cases}
$$

\footnotetext{
${ }^{46}$ The effects of imposing matter parity and $R$-parity in the MSSM are identical for all renormalizable interactions.
} 
The conservation of $R$-parity in scattering and decay processes has a critical impact on supersymmetric phenomenology. For example, any initial state in a scattering experiment will involve ordinary ( $R$-even) particles. Consequently, it follows that supersymmetric particles must be produced in pairs. In general, these particles are highly unstable and decay into lighter states. Moreover, $R$-parity invariance also implies that the lightest supersymmetric particle (LSP) is absolutely stable, and must eventually be produced at the end of a decay chain initiated by the decay of a heavy unstable supersymmetric particle.

In order to be consistent with cosmological constraints, a stable LSP is almost certainly electrically and color neutral. Consequently, the LSP in an $R$-parity-conserving theory is weakly interacting with ordinary matter, i.e, it behaves like a stable heavy neutrino and will escape collider detectors without being directly observed. Thus, the canonical signature for conventional $R$-parity-conserving supersymmetric theories is missing (transverse) energy, due to the escape of the LSP. Moreover, the stability of the LSP in $R$-parity-conserving supersymmetry makes it a promising candidate for dark matter.

\subsubsection{MSSM parameters of the SUSY-conserving sector}

The parameters of the SUSY-conserving sector consist of: (i) gauge couplings, $g_{s}, g$, and $g^{\prime}$, corresponding to the Standard Model gauge group $\mathrm{SU}(3) \times \mathrm{SU}(2) \times \mathrm{U}(1)$ respectively; (ii) a SUSY-conserving higgsino mass parameter $\mu$; and (iii) Higgs-fermion Yukawa coupling constants, $\lambda_{u}, \lambda_{d}$, and $\lambda_{e}$, corresponding to the couplings of one generation of left- and righthanded quarks and leptons and their superpartners to the Higgs bosons and higgsinos. Because there is no right-handed neutrino (or its superpartner) in the MSSM as defined here, a Yukawa coupling $\lambda_{\nu}$ is not included. The complex $\mu$ parameter and Yukawa couplings enter via the most general renormalizable $R$-parity-conserving superpotential given by eq. (8.3) with $W_{\mathrm{RPV}}=0$.

One can now obtain the scalar potential from eq. (6.66) as applied to the MSSM,

$$
V_{\text {scalar }}=\frac{1}{2}\left[D^{a} D^{a}+\left(D^{\prime}\right)^{2}\right]+F_{i}^{*} F_{i},
$$

where the index $a$ runs over the $\mathrm{SU}(3)$ and $\mathrm{SU}(2)$ gauge indices and $D^{\prime}$ is the $\mathrm{U}(1)_{Y} D$-term. Focusing on the terms that depend on the Higgs boson fields, one obtains, 
$V_{\mathrm{Higgs}}=|\mu|^{2}\left[\left|H_{d}\right|^{2}+\left|H_{u}\right|^{2}\right]+\frac{1}{8}\left(g^{2}+g^{\prime 2}\right)\left[\left|H_{d}\right|^{2}-\left|H_{u}\right|^{2}\right]^{2}+\frac{1}{2} g^{2}\left|H_{d}^{*} H_{u}\right|^{2}$.

Clearly $\left\langle V_{\text {Higgs }}\right\rangle \equiv\left\langle 0\left|V_{\text {Higgs }}\right| 0\right\rangle \geq 0$, as expected. Moreover, $H_{d}=H_{u}=0$ minimizes the Higgs scalar potential, which yields $\left\langle V_{\text {Higgs }}\right\rangle=0$, corresponding to a supersymmetric vacuum. Thus, there is no $\mathrm{SU}(2) \times \mathrm{U}(1)$ breaking at this stage. But after introducing soft SUSY-breaking terms, some of which involve the Higgs fields, it will then be possible to spontaneously break the $\mathrm{SU}(2) \times \mathrm{U}(1)$ symmetry. Consequently, SUSY breaking and electroweak symmetry breaking are intimately related in the MSSM.

\subsection{Supersymmetry breaking in the MSSM}

Following the rules of Girardello and Grisaru [136] that were presented in Section 7.4.1, we add the soft-SUSY-breaking terms, consistent with the $\mathrm{SU}(3) \times \mathrm{SU}(2) \times \mathrm{U}(1)$ gauge symmetry and the assumed $R$-parity invariance (for a review, see Ref. [43]). For simplicity, we consider in this section the case of one generation of quarks, leptons, and their scalar superpartners.

The supersymmetry-breaking sector contains the following sets of parameters: (i) three complex gaugino Majorana mass parameters, $M_{3}, M_{2}$, and $M_{1}$, associated with the $\mathrm{SU}(3), \mathrm{SU}(2)$, and $\mathrm{U}(1)$ subgroups of the Standard Model; (ii) five squark and slepton squared-mass parameters, $M_{\widetilde{Q}}^{2}, M_{\widetilde{U}}^{2}, M_{\widetilde{D}}^{2}, M_{\widetilde{L}}^{2}$, and $M_{\widetilde{E}}^{2}$, corresponding to the superpartners of the five electroweak multiplets of left-handed fermion fields and their chargeconjugates, $(u, d)_{L}, u_{L}^{c}, d_{L}^{c},\left(\nu, e^{-}\right)_{L}$, and $e_{L}^{c}$ [cf. Table 5]; and (iii) three Higgs-squark-squark and Higgs-slepton-slepton trilinear interaction terms, with complex coefficients $T_{U} \equiv \lambda_{u} A_{U}, T_{D} \equiv \lambda_{d} A_{D}$, and $T_{E} \equiv \lambda_{e} A_{E}$ (which define the $A$-parameters). Following Ref. [35], it is conventional to separate out the factors of the Yukawa couplings in defining the $A$-parameters, originally motivated by a simple class of gravity-mediated SUSY-breaking models $[31,38,132]$. With this definition, if the $A$-parameters are parametrically of the same order (or smaller) relative to other supersymmetrybreaking mass parameters, then only the third generation $A$-parameters will be phenomenologically relevant.

Finally, we have (iv) two real squared-mass parameters $\left(m_{1}^{2}\right.$ and $\left.m_{2}^{2}\right)$ and one complex squared-mass parameter, $m_{12}^{2} \equiv \mu B$ (the latter defines the $B$-parameter), which appear in the tree-level scalar Higgs potential,

$$
\begin{gathered}
V=\left(m_{1}^{2}+|\mu|^{2}\right) H_{d}^{\dagger} H_{d}+\left(m_{2}^{2}+|\mu|^{2}\right) H_{u}^{\dagger} H_{u}+\left(m_{12}^{2} H_{u} H_{d}+\text { h.c. }\right) \\
+\frac{1}{8}\left(g^{2}+g^{\prime 2}\right)\left(H_{d}^{\dagger} H_{d}-H_{u}^{\dagger} H_{u}\right)^{2}+\frac{1}{2}\left|H_{d}^{\dagger} H_{u}\right|^{2} .
\end{gathered}
$$


Note that the quartic Higgs couplings in eq. (8.11) are related to the gauge couplings $g$ and $g^{\prime}$ as a consequence of supersymmetry. The breaking of the electroweak symmetry $\mathrm{SU}(2) \times \mathrm{U}(1)$ to $\mathrm{U}(1)_{\mathrm{EM}}$ is only possible after introducing the supersymmetry-breaking Higgs squared-mass parameters $m_{1}^{2}, m_{2}^{2}$ (which can be negative) and $m_{12}^{2}$. After minimizing the Higgs scalar potential, these three squared-mass parameters can be re-expressed in terms of the two Higgs vacuum expectation values, $\left\langle H_{d}^{0}\right\rangle \equiv v_{d} / \sqrt{2}$ and $\left\langle H_{u}^{0}\right\rangle \equiv v_{u} / \sqrt{2}$, and the CP-odd Higgs mass $m_{A}$ [cf. eqs. (8.13) and (8.14) below]. One is always free to rephase the Higgs doublet fields such that $v_{d}$ and $v_{u}$ are both real and positive.

The quantity, $v_{d}^{2}+v_{u}^{2}=4 m_{W}^{2} / g^{2}=\left(2 G_{F}^{2}\right)^{-1 / 2} \simeq(246 \mathrm{GeV})^{2}$, is fixed by the Fermi constant, $G_{F}$, whereas the ratio

$$
\tan \beta=\frac{v_{u}}{v_{d}}
$$

is a free parameter such that $0 \leq \beta \leq \pi / 2$. The tree-level conditions for the scalar potential minimum relate the diagonal and off-diagonal Higgs squared-mass parameters in terms of $m_{Z}^{2}=\frac{1}{4}\left(g^{2}+g^{\prime 2}\right)\left(v_{d}^{2}+v_{u}^{2}\right)$, the angle $\beta$, and the CP-odd Higgs mass $m_{A}$ :

$$
\begin{gathered}
\sin 2 \beta=\frac{2 m_{12}^{2}}{m_{1}^{2}+m_{2}^{2}+2|\mu|^{2}}=\frac{2 m_{12}^{2}}{m_{A}^{2}}, \\
\frac{1}{2} m_{Z}^{2}=-|\mu|^{2}+\frac{m_{1}^{2}-m_{2}^{2} \tan ^{2} \beta}{\tan ^{2} \beta-1} .
\end{gathered}
$$

At this stage, one can already see the tension with naturalness, if the SUSY parameters, $\left|m_{1}\right|,\left|m_{2}\right|$ and $|\mu|$, are significantly larger than the scale of electroweak symmetry breaking. In this case, $m_{Z}^{2}$ will be the difference of two large numbers, requiring some fine-tuning of the SUSY parameters in order to produce the correct $Z$ boson mass. In the literature, this tension is referred to as the little hierarchy problem [93-95], previous noted in Section 3.3. One must also guard against the existence of charge and/or color breaking global minima due to non-zero vacuum expectation values for the squark and charged slepton fields. This possibility can be avoided if the $A$-parameters are not unduly large [154-160]. Additional constraints must also be respected to avoid directions in scalar field space in which the full tree-level scalar potential can become unbounded from below [160].

\subsection{The MSSM parameter count}

The total number of independent physical parameters that define the MSSM (in its most general form) is quite large, primarily due to the soft- 
supersymmetry-breaking sector. In particular, in the case of three generations of quarks, leptons, and their superpartners, $M_{\widetilde{Q}}^{2}, M_{\widetilde{U}}^{2}, M_{\widetilde{D}}^{2}, M_{\widetilde{L}}^{2}$, and $M_{\widetilde{E}}^{2}$ are hermitian $3 \times 3$ matrices, and $A_{U}, A_{D}$, and $A_{E}$ are complex $3 \times 3$ matrices. In addition, $M_{1}, M_{2}, M_{3}, B$, and $\mu$ are in general complex parameters. Finally, as in the Standard Model, the Higgs-fermion Yukawa couplings, $\lambda_{f}(f=u, d$, and $e)$, are complex $3 \times 3$ matrices that are related to the quark and lepton mass matrices via: $M_{f}=\lambda_{f} v_{f} / \sqrt{2}$, where $v_{e} \equiv v_{d}$ [with $v_{u}$ and $v_{d}$ as defined above eq. (8.12)].

However, not all these parameters are physical. Some of the MSSM parameters can be eliminated by expressing interaction eigenstates in terms of the mass eigenstates, with an appropriate redefinition of the MSSM fields to remove unphysical degrees of freedom. The analysis of Refs. [161, 162] shows that the MSSM possesses 124 independent parameters. Of these, 18 correspond to SM parameters (including the QCD vacuum angle, $\theta_{\mathrm{QCD}}$ ), one corresponds to a Higgs sector parameter (the analogue of the SM Higgs mass), and 105 are genuinely new parameters of the model. The latter include: five real parameters and three $\mathrm{CP}$-violating phases in the gaugino/higgsino sector, 21 squark and slepton masses, 36 real mixing angles to define the squark and slepton mass eigenstates, and $40 \mathrm{CP}$-violating phases that can appear in the squark and slepton interactions.

Unfortunately, without additional restrictions on the 124 parameters, the MSSM is not a phenomenologically viable theory. In particular, a generic point of the MSSM parameter space typically exhibits: (i) no conservation of the separate lepton numbers $L_{e}, L_{\mu}$, and $L_{\tau}$; (ii) unsuppressed flavor-changing neutral currents (FCNCs) [163, 164]; and (iii) new sources of $\mathrm{CP}$ violation [165] that are inconsistent with the experimental bounds. For example, the strong suppression of FCNCs observed in nature implies that the off-diagonal matrix elements of the soft-SUSY-breaking squark and slepton squared-mass matrices are highly constrained [43, 45].

In practice, various simplifying assumptions are imposed on the SUSYbreaking sector to reduce the number of parameters to a more manageable form, such that the constraints imposed by lepton and quark flavor changing and CP-violating processes are satisfied. For example, specific models of gravity-mediated and gauge-mediated supersymmetry breaking ${ }^{47}$ introduce a small number of fundamental parameters that provide the source for SUSY-breaking for the MSSM, consistent with the constraints due to flavor

${ }^{47}$ One of the benefits of GMSB models is that the SUSY-breaking is transmitted to the MSSM sector via gauge boson exchange, which is automatically flavor-conserving. 
and $\mathrm{CP}$ violation. More details can be found in Ref. [48].

An alternative approach, called the phenomenological MSSM (pMSSM) has been introduced [166, 167], which attempts to identify the parameters most relevant for phenomenology, subject to a number of simplifying assumptions. The pMSSM is governed by 19 independent real supersymmetric parameters: the three gaugino mass parameters $M_{1}, M_{2}$ and $M_{3}$, the Higgs sector parameters $m_{A}$ and $\tan \beta$, the Higgsino mass parameter $\mu$, five squark and slepton squared-mass parameters for the degenerate first and second generations $\left(M_{\widetilde{Q}}^{2}, M_{\widetilde{U}}^{2}, M_{\widetilde{D}}^{2}, M_{\widetilde{L}}^{2}\right.$ and $\left.M_{\widetilde{E}}^{2}\right)$, the five corresponding squark and slepton squared-mass parameters for the third generation, and three third-generation $A$-parameters $\left(A_{t}, A_{b}\right.$ and $\left.A_{\tau}\right) .{ }^{48}$ The first and second generation $A$-parameters can be neglected as their phenomenological consequences are negligible. Such an approach assumes that new sources of flavor violation and/or CP-violation are either absent or negligible. ${ }^{49}$

\subsection{The MSSM particle spectrum}

\subsubsection{Spin-1/2 superpartners}

The superpartners of the gauge and Higgs bosons are fermions, whose names are obtained by appending "ino" to the end of the corresponding SM particle name. The gluino is the color-octet Majorana fermion partner of the gluon with mass $M_{\widetilde{g}}=\left|M_{3}\right|$. The superpartners of the electroweak gauge and Higgs bosons (the gauginos and higgsinos) can mix due to $\mathrm{SU}(2) \times \mathrm{U}(1)$ breaking effects. As a result, the physical states of definite mass are modeldependent linear combinations of the charged or neutral gauginos and higgsinos, called charginos and neutralinos, respectively (sometimes collectively called electroweakinos). The charginos are Dirac fermions, and the neutralinos are Majorana fermions.

The tree-level mixing of the charged gauginos $\left(\widetilde{W}^{ \pm}\right)$and higgsinos $\left(\widetilde{H}_{u}^{+}\right.$ and $\widetilde{H}_{d}^{-}$) is governed by a $2 \times 2$ complex mass matrix,

$$
M_{C} \equiv\left(\begin{array}{cc}
M_{2} & g v_{u} / \sqrt{2} \\
g v_{d} / \sqrt{2} & \mu
\end{array}\right) .
$$

\footnotetext{
${ }^{48}$ In Ref. [168], the number of pMSSM parameters is reduced to ten by assuming one common squark mass parameter for the first two generations, a second common squark mass parameter for the third generation, a common slepton mass parameter, and a common third generation $A$ parameter.

${ }^{49}$ The pMSSM approach has been recently extended to include additional CP-violating SUSY-breaking parameters in Ref. [169].
} 
The physical chargino states and their masses are obtained by performing a singular value decomposition of the complex matrix $M_{C}$ [cf. eq. (2.86)]:

$$
U^{*} M_{C} V^{-1}=\operatorname{diag}\left(M_{\widetilde{\chi}_{1}^{+}}, M_{\widetilde{\chi}_{2}^{+}}\right),
$$

where $U$ and $V$ are unitary matrices. The physical chargino states are Dirac fermions and are denoted by $\widetilde{\chi}_{1}^{ \pm}$and $\widetilde{\chi}_{2}^{ \pm}$. These are linear combinations of the charged gaugino and higgsino states determined by the matrix elements of $U$ and $V$. The chargino masses correspond to the singular values of $M_{C}$, i.e., the positive square roots of the eigenvalues of $M_{C}^{\dagger} M_{C}$,

$$
\begin{aligned}
M_{\tilde{\chi}_{1}^{+}, \tilde{\chi}_{2}^{+}}^{2}=\frac{1}{2}\left\{|\mu|^{2}+\left|M_{2}\right|^{2}+2 m_{W}^{2}\right. & \\
& \left.\mp \sqrt{\left(|\mu|^{2}+\left|M_{2}\right|^{2}+2 m_{W}^{2}\right)^{2}-4\left|\mu M_{2}-m_{W}^{2} \sin 2 \beta\right|^{2}}\right\},
\end{aligned}
$$

where the states are ordered such that $M_{\widetilde{\chi}_{1}^{+}} \leq M_{\widetilde{\chi}_{2}^{+}}$. The relative phase of $\mu$ and $M_{2}$ is physical and potentially observable.

The tree-level mixing of the neutral gauginos $\left(\widetilde{B}\right.$ and $\left.\widetilde{W}^{0}\right)$ and higgsinos $\left(\widetilde{H}_{d}^{0}\right.$ and $\left.\widetilde{H}_{u}^{0}\right)$ is governed by a $4 \times 4$ complex symmetric mass matrix,

$$
M_{N} \equiv\left(\begin{array}{cccc}
M_{1} & 0 & -\frac{1}{2} g^{\prime} v_{d} & \frac{1}{2} g^{\prime} v_{u} \\
0 & M_{2} & \frac{1}{2} g v_{d} & -\frac{1}{2} g v_{u} \\
-\frac{1}{2} g^{\prime} v_{d} & \frac{1}{2} g v_{d} & 0 & -\mu \\
\frac{1}{2} g^{\prime} v_{u} & -\frac{1}{2} g v_{u} & -\mu & 0
\end{array}\right) .
$$

To determine the physical neutralino states and their masses, one must perform a Takagi-diagonalization of the complex symmetric matrix $M_{N}$ [cf. eq. (2.77)]:

$$
W^{T} M_{N} W=\operatorname{diag}\left(M_{\widetilde{\chi}_{1}^{0}}, M_{\widetilde{\chi}_{2}^{0}}, M_{\widetilde{\chi}_{3}^{0}}, M_{\widetilde{\chi}_{4}^{0}}\right),
$$

where $W$ is a unitary matrix. The physical neutralino states are Majorana fermions, and are denoted by $\widetilde{\chi}_{i}^{0}(i=1, \ldots 4)$, where the states are ordered such that $M_{\widetilde{\chi}_{1}^{0}} \leq M_{\widetilde{\chi}_{2}^{0}} \leq M_{\widetilde{\chi}_{3}^{0}} \leq M_{\widetilde{\chi}_{4}^{0}}$. The $\widetilde{\chi}_{i}^{0}$ are the linear combinations of the neutral gaugino and higgsino states determined by the matrix elements of $W$. The neutralino masses correspond to the singular values of $M_{N}$, i.e., the positive square roots of the eigenvalues of $M_{N}^{\dagger} M_{N}$.

\subsubsection{Spin-0 superpartners}

The superpartners of the quarks and leptons are spin-zero bosons: the squarks, charged sleptons, and sneutrinos, respectively. For a given Dirac fermion $f$, there are two superpartners, $\widetilde{f}_{L}$ and $\widetilde{f}_{R}$, where the $L$ and $R$ 
subscripts simply identify the scalar partners that are related by supersymmetry to the left-handed and right-handed fermions, $f_{L, R} \equiv \frac{1}{2}\left(1 \mp \gamma_{5}\right) f$, respectively. (There is no $\widetilde{\nu}_{R}$ in the MSSM.) However, $\widetilde{f}_{L}-\widetilde{f}_{R}$ mixing is possible, in which case $\widetilde{f}_{L}$ and $\widetilde{f}_{R}$ are not mass eigenstates.

We first consider the squarks and the sleptons. For three generations of squarks, one must diagonalize $6 \times 6$ matrices corresponding to the basis $\left(\widetilde{q}_{i L}, \widetilde{q}_{i R}\right)$, where $i=1,2,3$ are the generation labels. For simplicity, only the one-generation case is illustrated in detail below.

Using the notation of the third family, the one-generation tree-level squark squared-mass matrix is given by

$$
\mathcal{M}^{2}=\left(\begin{array}{cc}
M_{\widetilde{Q}}^{2}+m_{q}^{2}+L_{q} & m_{q} X_{q}^{*} \\
m_{q} X_{q} & M_{\widetilde{R}}^{2}+m_{q}^{2}+R_{q}
\end{array}\right),
$$

where

$$
X_{q} \equiv A_{q}-\mu^{*}(\cot \beta)^{2 T_{3 q}},
$$

and

$$
T_{3 q}=\left\{\begin{aligned}
\frac{1}{2}, & \text { for } q=t \\
-\frac{1}{2}, & \text { for } q=b .
\end{aligned}\right.
$$

The diagonal squared-masses are governed by soft-SUSY-breaking squared-masses $M_{\widetilde{Q}}^{2}$ and $M_{\widetilde{R}}^{2} \equiv M_{\widetilde{U}}^{2}\left[M_{\widetilde{D}}^{2}\right]$ for $q=t[b]$, the corresponding quark masses $m_{t}\left[m_{b}\right]$, and electroweak correction terms:

where $e_{q}=\frac{2}{3}\left[-\frac{1}{3}\right]$ for $q=t[b]$.

$$
\begin{aligned}
& L_{q} \equiv\left(T_{3 q}-e_{q} \sin ^{2} \theta_{W}\right) m_{Z}^{2} \cos 2 \beta, \\
& R_{q} \equiv e_{q} \sin ^{2} \theta_{W} m_{Z}^{2} \cos 2 \beta,
\end{aligned}
$$

The off-diagonal squark squared-masses are proportional to the corresponding quark masses and depend on $\tan \beta$, the soft-SUSY-breaking $A$-parameters and the higgsino mass parameter $\mu$. Assuming that the $A$ parameters are parametrically of the same order (or smaller) relative to other SUSY-breaking mass parameters, it then follows that $\widetilde{q}_{L}-\widetilde{q}_{R}$ mixing effects are small, with the possible exception of the third generation, where mixing can be enhanced by factors of $m_{t}$ and $m_{b} \tan \beta$.

In the case of third generation $\widetilde{q}_{L}-\widetilde{q}_{R}$ mixing, the mass eigenstates (denoted by $\widetilde{q}_{1}$ and $\widetilde{q}_{2}$, with $m_{\tilde{q}_{1}}<m_{\tilde{q}_{2}}$ ) are determined by diagonalizing the $2 \times 2$ matrix $\mathcal{M}^{2}$. The corresponding squared-masses and mixing angle are:

$$
\begin{aligned}
m_{\tilde{q}_{1,2}}^{2} & =\frac{1}{2}\left[\operatorname{Tr} \mathcal{M}^{2} \mp \sqrt{\left(\operatorname{Tr} \mathcal{M}^{2}\right)^{2}-4 \operatorname{det} \mathcal{M}^{2}}\right], \\
\sin 2 \theta_{\tilde{q}} & =\frac{2 m_{q}\left|X_{q}\right|}{m_{\tilde{q}_{2}}^{2}-m_{\tilde{q}_{1}}^{2}} .
\end{aligned}
$$


The results above also apply to the charged sleptons with the substitutions: $q \rightarrow \ell$ with $T_{3 \ell}=-\frac{1}{2}$ and $e_{\ell}=-1$, and the replacement of the SUSYbreaking parameters: $M_{\widetilde{Q}}^{2} \rightarrow M_{\widetilde{L}}^{2}, M_{\widetilde{D}}^{2} \rightarrow M_{\widetilde{E}}^{2}$, and $A_{q} \rightarrow A_{\tau}$. For the neutral sleptons, $\widetilde{\nu}_{R}$ does not exist in the MSSM, so $\widetilde{\nu}_{L}$ is a mass eigenstate.

In the case of three generations, the supersymmetry-breaking scalarsquared masses $\left[M_{\widetilde{Q}}^{2}, M_{\widetilde{U}}^{2}, M_{\widetilde{D}}^{2}, M_{\widetilde{L}}^{2}\right.$, and $\left.M_{\widetilde{E}}^{2}\right]$ and the $A$-parameters $\left[A_{U}\right.$, $A_{D}$, and $\left.A_{E}\right]$ are now $3 \times 3$ matrices. The diagonalization of the $6 \times 6$ squark mass matrices yields $\widetilde{f}_{i L}-\widetilde{f}_{j R}$ mixing (for $i \neq j$ ). In practice, since the $\widetilde{f}_{L}-\widetilde{f}_{R}$ mixing is appreciable only for the third generation, this additional complication can often be neglected.

\subsection{The Higgs sector of the MSSM}

Having completed our tour of the superpartners of the SM particles, we now focus of the Higgs sector of the MSSM [170-172]. We first provide details of the structure of the Higgs sector based on a tree-level analysis. We then discuss the importance of radiative corrections, in light of the observed Higgs boson with a mass of $125 \mathrm{GeV}$.

\subsubsection{The tree-level MSSM Higgs sector}

The tree-level scalar Higgs potential, previously given in eq. (8.11), is CPconserving. This follows from the fact that $m_{12}^{2}$, the only potentially complex parameter that appears in eq. (8.11), can be chosen real and positive by an appropriate rephasing of the Higgs fields.

After minimizing the Higgs potential, as indicated above eq. (8.12), one can identify the physical Higgs states. The five physical Higgs particles consist of a charged Higgs pair

$$
H^{ \pm}=H_{d}^{ \pm} \sin \beta+H_{u}^{ \pm} \cos \beta,
$$

one CP-odd neutral scalar

$$
A=\sqrt{2}\left(\operatorname{Im} H_{d}^{0} \sin \beta+\operatorname{Im} H_{u}^{0} \cos \beta\right),
$$

and two CP-even neutral scalar mass eigenstates that are determined by diagonalizing the neutral CP-even Higgs scalar squared-mass matrix,

$$
\mathcal{M}_{0}^{2}=\left(\begin{array}{cc}
m_{A}^{2} \sin ^{2} \beta+m_{Z}^{2} \cos ^{2} \beta & -\left(m_{A}^{2}+m_{Z}^{2}\right) \sin \beta \cos \beta \\
-\left(m_{A}^{2}+m_{Z}^{2}\right) \sin \beta \cos \beta & m_{A}^{2} \cos ^{2} \beta+m_{Z}^{2} \sin ^{2} \beta
\end{array}\right) .
$$

The eigenstates of $\mathcal{M}_{0}^{2}$ are identified as the neutral CP-even Higgs bosons,

$$
\begin{aligned}
h & =-\left(\sqrt{2} \operatorname{Re} H_{d}^{0}-v_{d}\right) \sin \alpha+\left(\sqrt{2} \operatorname{Re} H_{u}^{0}-v_{u}\right) \cos \alpha, \\
H & =\left(\sqrt{2} \operatorname{Re} H_{d}^{0}-v_{d}\right) \cos \alpha+\left(\sqrt{2} \operatorname{Re} H_{u}^{0}-v_{u}\right) \sin \alpha,
\end{aligned}
$$


which defines the CP-even Higgs mixing angle $\alpha$.

All Higgs masses and couplings can be expressed in terms of two parameters, usually chosen to be $m_{A}$ and $\tan \beta$. The charged Higgs mass is given by

$$
m_{H^{ \pm}}^{2}=m_{A}^{2}+m_{W}^{2} .
$$

The squared-masses of the CP-even Higgs bosons $h$ and $H$ are eigenvalues of $\mathcal{M}_{0}^{2}$. The trace and determinant of $\mathcal{M}_{0}^{2}$ yield,

$$
m_{h}^{2}+m_{H}^{2}=m_{A}^{2}+m_{Z}^{2}, \quad m_{h}^{2} m_{H}^{2}=m_{A}^{2} m_{Z}^{2} \cos ^{2} 2 \beta,
$$

where the CP-even Higgs masses are given by

$$
m_{H, h}^{2}=\frac{1}{2}\left(m_{A}^{2}+m_{Z}^{2} \pm \sqrt{\left(m_{A}^{2}+m_{Z}^{2}\right)^{2}-4 m_{Z}^{2} m_{A}^{2} \cos ^{2} 2 \beta}\right) .
$$

In the convention where $0 \leq \beta \leq \frac{1}{2} \pi$, it is standard practice to choose $\alpha$ to lie in the range $|\alpha| \leq \frac{1}{2} \pi$. However, because the off-diagonal element of $\mathcal{M}_{0}^{2}$ is negative semi-definite, one finds that $-\frac{1}{2} \pi \leq \alpha \leq 0$. More explicitly, the mixing angle $\alpha$ can be determined as a function of $m_{A}$ and $\tan \beta$ from the following expression and from eq. (8.34), ${ }^{50}$

$$
\cos \alpha=\sqrt{\frac{m_{A}^{2} \sin ^{2} \beta+m_{Z}^{2} \cos ^{2} \beta-m_{h}^{2}}{m_{H}^{2}-m_{h}^{2}}},
$$

and $\sin \alpha=-\left(1-\cos ^{2} \alpha\right)^{1 / 2}$.

In the expression for the couplings of the Higgs bosons with the gauge bosons, only the combination $\beta-\alpha$ appears. For example, the coupling of $h$ to $V V$ (where $V V=W^{+} W^{-}$or $Z Z$ ) relative to the corresponding coupling of the SM Higgs boson, $h_{\mathrm{SM}}$, is given by,

$$
\frac{g_{h V V}}{g_{h_{\mathrm{SM}} V V}}=\sin (\beta-\alpha) \text {. }
$$

Given the range of the angles $\alpha$ and $\beta$, it follows that $0 \leq \beta-\alpha \leq \pi$. In particular, the following expressions can be obtained,

$$
\begin{aligned}
\cos (\beta-\alpha) & =\frac{m_{Z}^{2} \sin 2 \beta \cos 2 \beta}{\sqrt{\left(m_{H}^{2}-m_{h}^{2}\right)\left(m_{H}^{2}-m_{Z}^{2} \cos ^{2} 2 \beta\right)}} . \\
\sin (\beta-\alpha) & =\sqrt{\frac{m_{H}^{2}-m_{Z}^{2} \cos ^{2} 2 \beta}{m_{H}^{2}-m_{h}^{2}}} .
\end{aligned}
$$

${ }^{50}$ The corresponding expressions for a general CP-conserving two Higgs doublet model can be found in Ref. [173] . 
One can check that eqs. (8.37) and (8.38) are consistent in light of eq. (8.33).

The Higgs-fermion Yukawa couplings are obtained from the MSSM superpotential [eq. (8.3) with $W_{\mathrm{RPV}}=0$ ] by employing the last two terms of eq. (6.63). Focusing on the Higgs interactions with third generation quarks, one obtains the so-called Type-II Higgs-quark interaction [174],

$$
\mathscr{L}_{\text {Yuk }}=-\epsilon^{i j}\left[h_{b} \bar{b}_{R} H_{d i} Q_{L j}+h_{t} \bar{t}_{R} Q_{L i} H_{u j}\right]+\text { h.c. },
$$

where $Q_{L} \equiv\left(t_{L}, b_{L}\right)$ is the quark doublet and $i$ and $j$ are $\mathrm{SU}(2)$ indices. In eq. (8.39), we employ four-component quark fields, where $q_{R, L} \equiv P_{R, L} q$ and $P_{R, L}=\frac{1}{2}\left(1 \pm \gamma_{5}\right)$. The quark masses are identified by replacing the Higgs fields in eq. (8.39) with their corresponding vacuum expectation values,

$$
m_{b}=h_{b} v \cos \beta / \sqrt{2}, \quad m_{t}=h_{t} v \sin \beta / \sqrt{2} .
$$

The tree-level Yukawa couplings of the lightest CP-even Higgs boson to third generation quark pairs are given by

$$
\begin{gathered}
g_{h b \bar{b}}=-\frac{m_{b}}{v} \frac{\sin \alpha}{\cos \beta}=\frac{m_{b}}{v}[\sin (\beta-\alpha)-\cos (\beta-\alpha) \tan \beta], \\
g_{h t \bar{t}}=\frac{m_{t}}{v} \frac{\cos \alpha}{\sin \beta}=\frac{m_{t}}{v}[\sin (\beta-\alpha)+\cos (\beta-\alpha) \cot \beta] .
\end{gathered}
$$

It is straightforward to work out the couplings of the other Higgs bosons of the model to the quarks (and leptons). A comprehensive set of Feynman rules for Higgs bosons in the MSSM can be found in Refs. [170, 171].

In the limit of $m_{A} \gg m_{Z}$, the expressions for the Higgs masses and mixing angle are given by,

$$
\begin{aligned}
m_{h}^{2} & \simeq m_{Z}^{2} \cos ^{2} 2 \beta-\frac{m_{Z}^{4} \sin ^{2} 4 \beta}{4 m_{A}^{2}}, \\
m_{H}^{2} & \simeq m_{A}^{2}+m_{Z}^{2} \sin ^{2} 2 \beta, \\
m_{H^{ \pm}}^{2} & =m_{A}^{2}+m_{W}^{2}, \\
\cos (\beta-\alpha) & \simeq \frac{m_{Z}^{2} \sin 4 \beta}{2 m_{A}^{2}} .
\end{aligned}
$$

Two consequences are immediately apparent. First,

$$
m_{A} \simeq m_{H} \simeq m_{H^{ \pm}}
$$

up to corrections of $\mathcal{O}\left(m_{Z}^{2} / m_{A}\right)$. Second, $\cos (\beta-\alpha) \simeq 0$, up to corrections of $\mathcal{O}\left(m_{Z}^{2} / m_{A}^{2}\right)$. This is the decoupling limit of the MSSM Higgs sector, since at energy scales below the approximately common mass of the heavy Higgs bosons $H^{ \pm}, H$, and $A^{0}$, the effective Higgs theory is equivalent to the one-doublet Higgs sector of the SM $[175,176]$. Indeed, one can check that in the limit of $\cos (\beta-\alpha) \rightarrow 0$, all the $h$ couplings to SM particles approach their SM limits, as in the case of the $h V V$ coupling exhibited in eq. (8.36) and in the case of the $h q \bar{q}$ couplings exhibited in eqs. (8.41) and (8.42). 


\subsubsection{Impact of radiative corrections on the MSSM Higgs sector}

The tree-level result for $m_{h}$ given in eq. (8.34) yields a startling prediction,

$$
m_{h} \leq m_{Z}|\cos 2 \beta| \leq m_{Z} \text {. }
$$

This is clearly in conflict with the observed Higgs mass of $125 \mathrm{GeV}$. However, the above inequality receives quantum corrections. The Higgs mass can be shifted due to loops of particles and their superpartners exhibited below (an incomplete cancellation, which would have been exact if supersymmetry were unbroken).
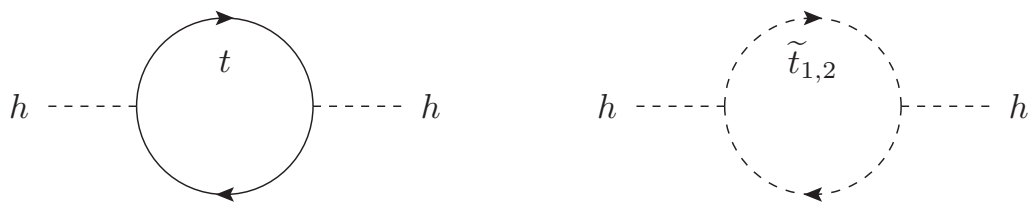

The impact of these corrections can be significant [177-179]. In particular, the qualitative behavior of the one-loop radiative corrections can be most easily seen in the limit of large top-squark masses. In this limit, both the off-diagonal entries and the splitting between the two diagonal entries of the top-squark squared-mass matrix [eq. (8.20)] are small in comparison to the square of the geometric mean of the two top-squark masses, $M_{\mathrm{S}}^{2} \equiv M_{\widetilde{t}_{1}} M_{\widetilde{t}_{2}}$. In this case (assuming $m_{A}>m_{Z}$ ), the predicted upper bound for $m_{h}$ is approximately given by [180]

$$
m_{h}^{2} \lesssim m_{Z}^{2}+\frac{3 g^{2} m_{t}^{4}}{8 \pi^{2} m_{W}^{2}}\left[\ln \left(\frac{M_{S}^{2}}{m_{t}^{2}}\right)+\frac{X_{t}^{2}}{M_{S}^{2}}\left(1-\frac{X_{t}^{2}}{12 M_{S}^{2}}\right)\right],
$$

where $X_{t} \equiv A_{t}-\mu \cot \beta$ governs stop mixing (taking $A_{t}$ and $\mu$ real for simplicity). The Higgs mass upper limit is saturated when $\tan \beta$ is large [i.e., $\left.\cos ^{2}(2 \beta) \sim 1\right]$ and $X_{t}=\sqrt{6} M_{S}$, which defines the so-called maximal mixing scenario.

A more complete treatment of the radiative corrections [181] shows that eq. (8.49) somewhat overestimates the true upper bound of $m_{h}$. These more refined computations, which incorporate renormalization group improvement, and the two-loop and leading three-loop contributions, yield an upper bound of $m_{h} \lesssim 135 \mathrm{GeV}$ in the region of large $\tan \beta$ (with an accuracy of a few $\mathrm{GeV}$ ) for $m_{t}=175 \mathrm{GeV}$ and $M_{S} \lesssim 2 \mathrm{TeV}$ [181], which is quite close to the observed value of the Higgs mass!

In certain cases, radiative corrections also can significantly modify the tree-level Yukawa couplings. For a review of such effects, see e.g., Ref. [182]. 


\subsection{Unification of gauge couplings}

Grand unification theory (GUT) predicts the unification of gauge couplings at some very high energy scale $[29,68,147,183]$. The running of the couplings is dictated by the particle content of the effective theory that resides below the GUT scale. However, attempts to embed the Standard Model in an $\mathrm{SU}(5)$ or $\mathrm{SO}(10)$ unified theory do not quite succeed. In particular, the three running gauge couplings (the strong QCD coupling $g_{s}$ and the electroweak gauge couplings $g$ and $g^{\prime}$ ) do not meet at a point, as shown by the dashed lines in Fig. 1. In contrast, in the case of the MSSM with superpartner masses of order $1 \mathrm{TeV}$, the renormalization group evolution is modified above the SUSY-breaking scale. In this case, unification of gauge couplings can be (approximately) achieved as illustrated by the red and blue lines in Fig. 1.

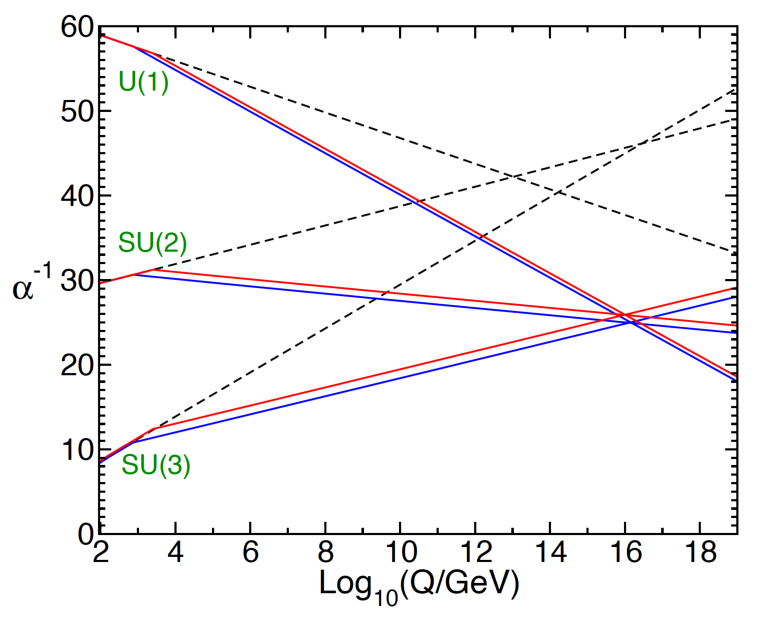

Fig. 1.: Renormalization group evolution of the inverse gauge couplings $\alpha_{a}^{-1}(Q)$ in the Standard Model (dashed lines) and the MSSM (solid lines). In the MSSM case, $\alpha_{3}\left(m_{Z}\right)$ is varied between 0.121 and 0.117 , and the supersymmetric particle mass thresholds are between $500 \mathrm{GeV}$ and $1.5 \mathrm{TeV}$, for the lower and upper solid lines, respectively. Two-loop effects are included. Taken from Ref. [38].

A quantitative assessment of the success of gauge coupling unification can be performed as follows. Since the electroweak gauge couplings $g$ and $g^{\prime}$ are very well measured, first focus on these two couplings. For a given lowenergy effective theory (below the GUT scale), we use the renormalization group equations (RGEs) to determine the couplings $g$ and $g^{\prime}$ as a function 
of the energy scale. We then define $M_{\mathrm{GUT}}$ to be the scale at which these two couplings meet.

We now assume that the unification of the three gauge couplings, $g_{s}, g$ and $g^{\prime}$ occurs at $M_{\mathrm{GUT}}$. Using the RGEs for the gauge couplings, we can now run $g_{s}$ down to the electroweak scale and compare with the experimentally measured value.

\subsubsection{Normalization of the $U(1)_{\mathrm{Y}}$ coupling}

In electroweak theory, the overall normalization of the $\mathrm{U}(1)_{\mathrm{Y}}$ coupling is a matter of convention. But, if the GUT group is simple and nonabelian, then the relative normalization of the $\mathrm{U}(1)_{\mathrm{Y}}$ coupling to the $\mathrm{SU}(2)$ gauge coupling is fixed. We denote the $\mathrm{SU}(3) \times \mathrm{SU}(2) \times \mathrm{U}(1)_{\mathrm{Y}}$ gauge couplings using the proper GUT normalization by $g_{3}, g_{2}$ and $g_{1}$ respectively. Our task is to relate $g_{1}$ with $g^{\prime}$. To do so, let us begin by considering the covariant derivative,

$$
D_{\mu}=\partial_{\mu}+i \sum_{a} g_{a} T^{a} A_{\mu}^{a} .
$$

If the gauge group is a direct product group, then different sets of generators $T^{a}$ are associated with with the different group factors, and we must use the appropriate $g_{a}$ depending on which generator it multiplies. In particular, for $\mathrm{SU}(2) \times \mathrm{U}(1)_{\mathrm{Y}}$ (below the GUT scale),

$$
g_{a} T^{a} A_{\mu}^{q} \ni g T^{3} W_{\mu}^{3}+g^{\prime} \frac{Y}{2} B_{\mu} .
$$

Above the GUT scale, the corresponding terms of the covariant derivative are

$$
g_{a} T^{a} A_{\mu}^{q} \ni g_{U}\left(T^{3} W_{\mu}^{3}+T^{0} B_{\mu}\right),
$$

where $g_{U}$ is the gauge coupling of the unifying GUT group and $T^{0}$ is the properly normalized hypercharge generator. In particular, the generators of the GUT group satisfy

$$
\operatorname{Tr}\left(T^{a} T^{b}\right)=T(R) \delta^{a b},
$$

where $T(R)$ is a constant that depends on the representation $R \cdot{ }^{51}$ We now set the two covariant derivatives above equal at the GUT scale,

$$
g_{U}\left(T^{3} W_{\mu}^{3}+T^{0} B_{\mu}\right)=g T^{3} W_{\mu}^{3}+g^{\prime} \frac{Y}{2} B_{\mu} .
$$

\footnotetext{
${ }^{51}$ Once $T(R)$ is fixed for one representation, it is then determined for all other representations. It is standard practice to fix $T(R)=\frac{1}{2}$ for the defining (fundamental) representation, although the argument presented below is independent of this choice.
} 
Table 6.: The $T_{3}$ and $Y$ quantum numbers of the two-component fermion fields that make up one generation of SM fermions. In computing the corresponding traces, one must not forget the color factor of 3 that arises when tracing over the (suppressed) color index.

\begin{tabular}{|crrcc|}
\hline Two-component fields & $T_{3}$ & $Y$ & $\operatorname{Tr}\left(T^{3}\right)^{2}$ & $\operatorname{Tr} Y^{2}$ \\
\hline$\psi_{Q_{1}}$ & $\frac{1}{2}$ & $\frac{1}{3}$ & $3\left(\frac{1}{4}\right)$ & $3\left(\frac{1}{9}\right)$ \\
$\psi_{Q_{2}}$ & $-\frac{1}{2}$ & $\frac{1}{3}$ & $3\left(\frac{1}{4}\right)$ & $3\left(\frac{1}{9}\right)$ \\
$\psi_{U}$ & 0 & $-\frac{4}{3}$ & $3(0)$ & $3\left(\frac{16}{9}\right)$ \\
$\psi_{D}$ & 0 & $\frac{2}{3}$ & $3(0)$ & $3\left(\frac{4}{9}\right)$ \\
$\psi_{L_{1}}$ & $\frac{1}{2}$ & -1 & $\frac{1}{4}$ & 1 \\
$\psi_{L_{2}}$ & $-\frac{1}{2}$ & -1 & $\frac{1}{4}$ & 1 \\
$\psi_{E}$ & 0 & 2 & 0 & 4 \\
\hline
\end{tabular}

Noting that $g_{U}=g_{3}=g_{2}=g_{1}$ at the GUT scale, it follows that $g_{2}=g$ and $g_{1} T^{0}=g^{\prime}(Y / 2)$. Since $T(R)$ only depends on the representation $R$, eq. (8.53) yields $\operatorname{Tr}\left(T^{3}\right)^{2}=\operatorname{Tr}\left(T^{0}\right)^{2}$. Thus,

$$
g_{1}^{2}=g^{\prime 2} \frac{\operatorname{Tr} Y^{2}}{4 \operatorname{Tr}\left(T^{3}\right)^{2}} .
$$

The relevant quantum numbers are provided in Table 6 .

The traces in eq. (8.55) are evaluated by summing over one generation of fermions, under the assumption that it is made up of complete irreducible representations of the GUT group. ${ }^{52}$ Using the results of Table 6, we simply add up the last two columns. Including the appropriate color factor of 3 when tracing over the suppressed color index, we obtain $\operatorname{Tr}\left(T^{3}\right)^{2}=2$ and $\operatorname{Tr} Y^{2}=\frac{40}{3}$. Thus, eq. (8.55) yields

$$
g_{1}^{2}=\frac{5}{3} g^{\prime 2}
$$

\subsubsection{Gauge coupling running}

We now examine the running of the gauge couplings in the one-loop approximation, where the gauge couplings $g_{i}$ obey the differential equation,

$$
\frac{d g_{i}^{2}}{d t}=\frac{b_{i} g_{i}^{4}}{16 \pi^{2}}, \quad \text { for } i=1,2,3
$$

\footnotetext{
${ }^{52} \mathrm{In}$ an $\mathrm{SU}(5) \mathrm{GUT}$, one generation of fermions make up a 10-dimensional and the complex conjugate of a 5-dimensional representation of $\mathrm{SU}(5)$. In an $\mathrm{SO}(10) \mathrm{GUT}$, one generation of fermions (including the right-handed neutrino) comprise a 16 dimensional spinor representation of $\mathrm{SO}(10)$.
} 
where $t=\ln Q^{2}$ ( $Q$ is the energy scale) and the $b_{i}$ are given by

$$
b_{i}=\frac{2}{3} \sum_{j} T\left(R_{j}^{(i)}\right) m\left(R_{j}^{(i)}\right)+\frac{1}{6} \sum_{J} c_{J} T\left(R_{J}^{(i)}\right) m\left(R_{J}^{(i)}\right)-\frac{11}{3} C_{A}\left(G^{(i)}\right),
$$

where the indices $j$ and $J$ are employed for two-component fermions and scalars, respectively. We have assumed that the low-energy gauge group is a direct product group, $G \equiv \prod_{i} G^{(i)}$ [where $G^{(i)}$ is either a simple compact Lie group or $\mathrm{U}(1)]$, and the $j$ th two-component fermion multiplet and the $J$ th scalar multiplet transform irreducibly under $G$ as $\left(R_{j}^{(1)}, R_{j}^{(2)}, \ldots\right)$ and $\left(R_{J}^{(1)}, R_{J}^{(2)}, \ldots\right)$, respectively. The multiplicity factors in eq. (8.58) are given by $m\left(R^{(i)}\right)=\prod_{k \neq i} d\left(R^{(k)}\right)$, where $d\left(R^{(k)}\right)$ is the dimension of the irreducible representation $R^{(k)}$, and $c_{J}=1\left[c_{J}=2\right]$ for real [complex] scalars. Finally, $T\left(R^{(i)}\right)$ is defined in eq. (8.53) in a convention where $T(R)=\frac{1}{2}$ for the defining representation of a simple compact Lie group, and $C_{A}\left(G^{(i)}\right)$ is the eigenvalue of the Casimir operator in the adjoint representation of $G^{(i)}$, which is defined in terms of the structure constants of the Lie group,

$$
f_{a b c} f_{a b d}=C_{A}(G) \delta_{c d} .
$$

For example, $C_{A}(\mathrm{G})=N$ for $\mathrm{G}=\mathrm{SU}(N)$. Note that for $\mathrm{U}(1)_{Y}$, we have $C_{A}(\mathrm{G})=0$ and

$$
T(R)=\left[\sqrt{\frac{3}{5}} \frac{1}{2} Y\right]^{2}=\frac{3}{20} Y^{2},
$$

where we have employed the properly normalized hypercharge generator, $\sqrt{3 / 5}(Y / 2)$.

The solution to eq. (8.57) is

$$
\frac{1}{g_{i}^{2}\left(m_{Z}\right)}=\frac{1}{g_{U}^{2}}-\frac{b_{i}}{16 \pi^{2}} \ln \left(\frac{m_{Z}^{2}}{M_{\mathrm{GUT}}^{2}}\right)
$$

where $M_{\mathrm{GUT}}$ is the GUT scale at which the three gauge couplings unify. Using eq. (8.61), the following two equations are obtained:

$$
\begin{aligned}
\sin ^{2} \theta_{W}\left(m_{Z}\right) & =\frac{g^{\prime 2}\left(m_{Z}\right)}{g^{2}\left(m_{Z}\right)+g^{\prime 2}\left(m_{Z}\right)}=\frac{\frac{3}{5} g_{1}^{2}\left(m_{Z}\right)}{g^{2}\left(m_{Z}\right)+\frac{3}{5} g_{1}^{2}\left(m_{Z}\right)} \\
& =\frac{3}{8}-\frac{5}{32 \pi} \alpha\left(m_{Z}\right)\left(b_{1}-b_{2}\right) \ln \left(\frac{M_{\mathrm{GUT}}^{2}}{m_{Z}^{2}}\right), \\
\ln \left(\frac{M_{\mathrm{GUT}}^{2}}{m_{Z}^{2}}\right) & =\frac{32 \pi}{5 b_{1}+3 b_{2}-8 b_{3}}\left(\frac{3}{8 \alpha\left(m_{Z}\right)}-\frac{1}{\alpha_{s}\left(m_{Z}\right)}\right),
\end{aligned}
$$

where $e=g \sin \theta_{W}, \alpha \equiv e^{2} / 4 \pi$ and $\alpha_{s} \equiv g_{s}^{2} / 4 \pi$. 
It is convenient to introduce the parameter,

$$
x \equiv \frac{1}{5}\left(\frac{b_{2}-b_{3}}{b_{1}-b_{2}}\right) .
$$

Then, eqs. (8.62) and (8.63) yield,

$$
\sin ^{2} \theta_{W}\left(m_{Z}\right)=\frac{1}{1+8 x}\left[3 x+\frac{\alpha\left(m_{Z}\right)}{\alpha_{s}\left(m_{Z}\right)}\right] .
$$

Once we know the value of $x$, we can use the above equation to determine $\alpha_{s}\left(m_{Z}\right)$ given the values of $\sin ^{2} \theta_{W}$ and $\alpha$, evaluated at $m_{Z}$,

$$
\alpha_{s}\left(m_{Z}\right)=\frac{\alpha\left(m_{Z}\right)}{(1+8 x) \sin ^{2} \theta_{W}\left(m_{Z}\right)-3 x} .
$$

The value of $x$ is determined from the values of the $b_{i}$, which are given by eq. (8.58).

One can now assess the success or failure of gauge coupling unification in the SM and in the MSSM. For details, see Problems 47 and 48. As advertised in Fig. 1, the gauge couplings do not unify when the SM is extrapolated to the GUT scale. In contrast, in the MSSM, the modified running of the gauge couplings due to the supersymmetric partners of the SM particles results in approximate unification. ${ }^{53}$ This success has often been touted as one of the motivations for $\mathrm{TeV}$-scale supersymmetry.

\subsection{Problems}

Problem 44. Starting with the SUSY Lagrangian for SUSY Yang Mills theory coupled to matter given in eq. (6.63), eliminate the auxiliary fields and obtain the Lagrangian of the MSSM prior to SUSY-breaking. For simplicity, you may consider only one generation of quarks and leptons and their superpartners. Then add in the soft-SUSY-breaking terms to obtain the complete MSSM Lagrangian. Using this result, verify the mass spectrum of the supersymmetric particles obtained in Section 8.5.

Problem 45. Using the results of Problem 44, verify the results obtained in Section 8.6 for the MSSM Higgs sector. Write out the Feynman rules for the interaction of the Higgs bosons with the gauge bosons and with the quarks and leptons.

\footnotetext{
${ }^{53}$ For a more precise analysis, we should extend the calculations of this subsection to include two-loop running of the gauge couplings [184]. One must also properly treat threshold corrections at the TeV scale $[185,186]$ (due to mass splittings among superpartners) and at the GUT scale [187]. The latter are quite model-dependent and allows some wiggle room in achieving precise gauge coupling unification.
} 
Problem 46. Using the results of Problem 44, one can obtain the complete set of Feynman rules for the MSSM with one generation of quarks and leptons and their superpartners. Work out as many of the rules as you can and check your results against Ref. [188].

Problem 47. Assuming $N_{g}$ generations of the quarks and leptons and $N_{h}$ copies of the SM Higgs boson, use eq. (8.58) to obtain

$$
\begin{aligned}
& b_{3}=\frac{4}{3} N_{g}-11, \\
& b_{2}=\frac{1}{6} N_{h}+\frac{4}{3} N_{g}-\frac{22}{3}, \\
& b_{1}=\frac{1}{10} N_{h}+\frac{4}{3} N_{g} .
\end{aligned}
$$

For the $S M$, we have $N_{g}=3$ and $N_{h}=1$. Check that $b_{3}=-7, b_{2}=-\frac{19}{6}$ and $b_{1}=\frac{41}{10}$. Consequently, independently of the value of $N_{g}$,

$$
x=\frac{23}{218}=0.1055 .
$$

Problem 48. Show that the SM results of Problem 47 are modified in the MSSM as follows:

$$
\begin{aligned}
& b_{3}=2 N_{g}-9, \\
& b_{2}=\frac{1}{2} N_{h}+2 N_{g}-6, \\
& b_{1}=\frac{3}{10} N_{h}+2 N_{g} .
\end{aligned}
$$

For the MSSM, we have $N_{g}=3$ and $N_{h}=2$. Verify that $b_{3}=-3, b_{2}=1$ and $b_{1}=\frac{33}{5}$, and consequently, $x=\frac{1}{7}$. Using the values for $\alpha\left(m_{Z}\right)$ and $\sin ^{2} \theta_{W}\left(m_{Z}\right)$ given in Ref. [189], evaluate $\alpha_{s}$ using eq. (8.66). Show that for $x=\frac{1}{7}$ (as predicted by the MSSM), one obtains a value for $\alpha_{s}\left(m_{Z}\right)$ that is quite close to the current world average [189]. Using $x=0.1055$, check that the corresponding $S M$ prediction for $\alpha_{s}\left(m_{Z}\right)$ is significantly lower than the observed value.

\section{Supersymmetry Quo Vadis?}

In these lectures, time constraints have limited the number of topics that we have been able to cover. The reader can consult the many fine books [2-29] and the reviews and lecture notes [30-49] already cited in Section 1 to pursue various topics in supersymmetry in greater depth.

In Section 8, we introduced the basics of the MSSM. But this is not the only possible supersymmetric extension of the SM. For example, in the MSSM as defined in Section 8, the neutrino is massless. There are 
a number of ways to extend the MSSM to allow for massive neutrinos. For example, by relaxing the assumption of $R$-parity conservation, one can introduce lepton number violating terms in the MSSM Lagrangian that can be used to incorporate massive neutrinos that are consistent with the neutrino oscillation data. ${ }^{54}$ Alternatively, one can start with the seesawextended SM and consider its supersymmetric extension [146, 193-201].

Extensions of the MSSM have also been proposed to solve a variety of theoretical problems. One such problem involves the $\mu$ parameter of the MSSM. Although $\mu$ is a SUSY-preserving parameter, it must be of order the effective SUSY-breaking scale of the MSSM to yield a consistent supersymmetric phenomenology [202]. Any natural solution to the so-called $\mu$-problem must incorporate a symmetry that enforces $\mu=0$ and a small symmetry-breaking parameter that generates a value of $\mu$ that is not parametrically larger than the effective SUSY-breaking scale [203].

A number of proposed mechanisms in the literature provide concrete examples of a natural solution to the $\mu$-problem of the MSSM (see, e.g., Refs. [202-206]). For example, one can replace $\mu$ by the vacuum expectation value of a new $\mathrm{SU}(3) \times \mathrm{SU}(2) \times \mathrm{U}(1)$ singlet scalar field. This can be achieved by adding a singlet chiral superfield to the MSSM. The end result is the next-to-minimal supersymmetric extension of the SM, otherwise known as the NMSSM, which is reviewed in Refs. [207, 208].

Ultimately, in order to determine how nature chooses to incorporate supersymmetry, one must discover evidence for supersymmetric particles in experiments. The phenomenology of the MSSM and its extensions is a huge subject that requires a separate lecture course. Since we have no time to present a detailed treatment of supersymmetric phenomenology here, we can only refer the reader to some of the excellent books and review articles on this subject (see e.g., Refs. [16, 17, 32, 47, 92]).

As discussed in Section 3, supersymmetry was proposed to avoid quadratic UV-sensitivity in a theory with elementary scalars. To avoid a significant fine-tuning of the fundamental parameters, which is required to explain the observed Higgs and $Z$ boson masses, the SUSY-breaking scale should be not much larger than $1 \mathrm{TeV}$. Consequently, experiments currently being carried out at the Large Hadron Collider (LHC) should be on the verge of discovering supersymmetric particles. However, so far no evidence for SUSY has emerged from the LHC data.

Figs. 2 and 3 summarize the limits on supersymmetric particle masses

${ }^{54}$ There is a huge literature on this subject. See, e.g., Refs. [190-192] and the references contained therein. 


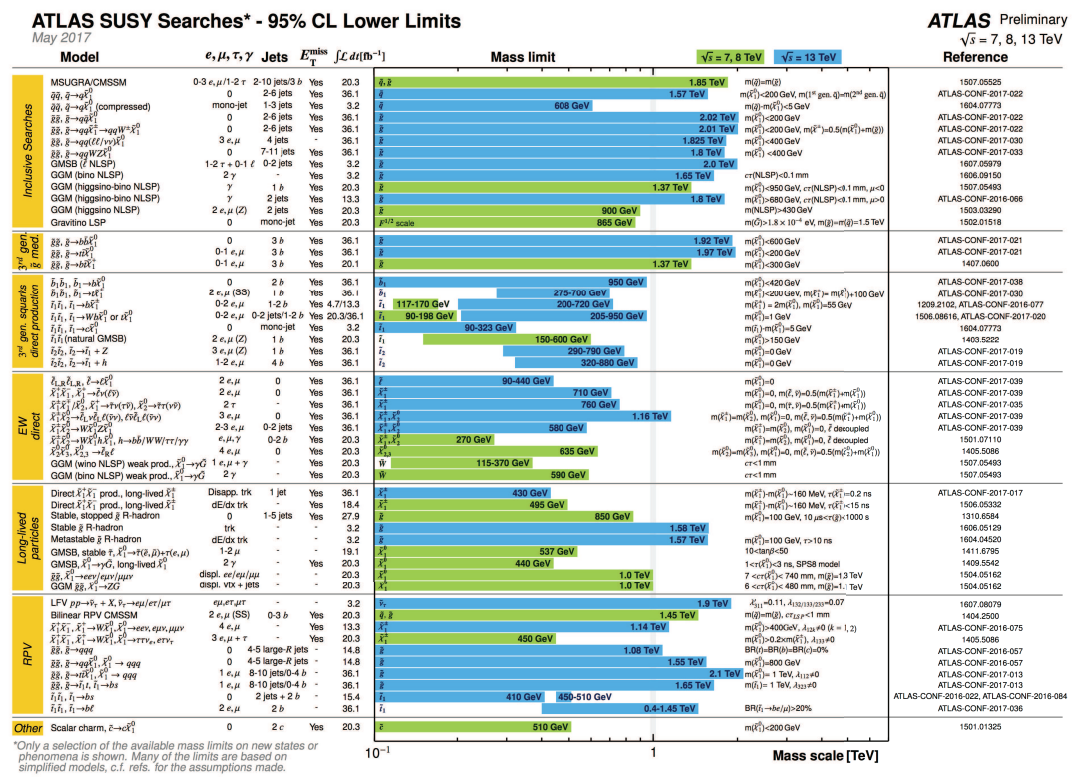

Fig. 2.: Mass reach of a representative selection of ATLAS searches for SUSY as of May, 2017. Taken from Ref. [209].

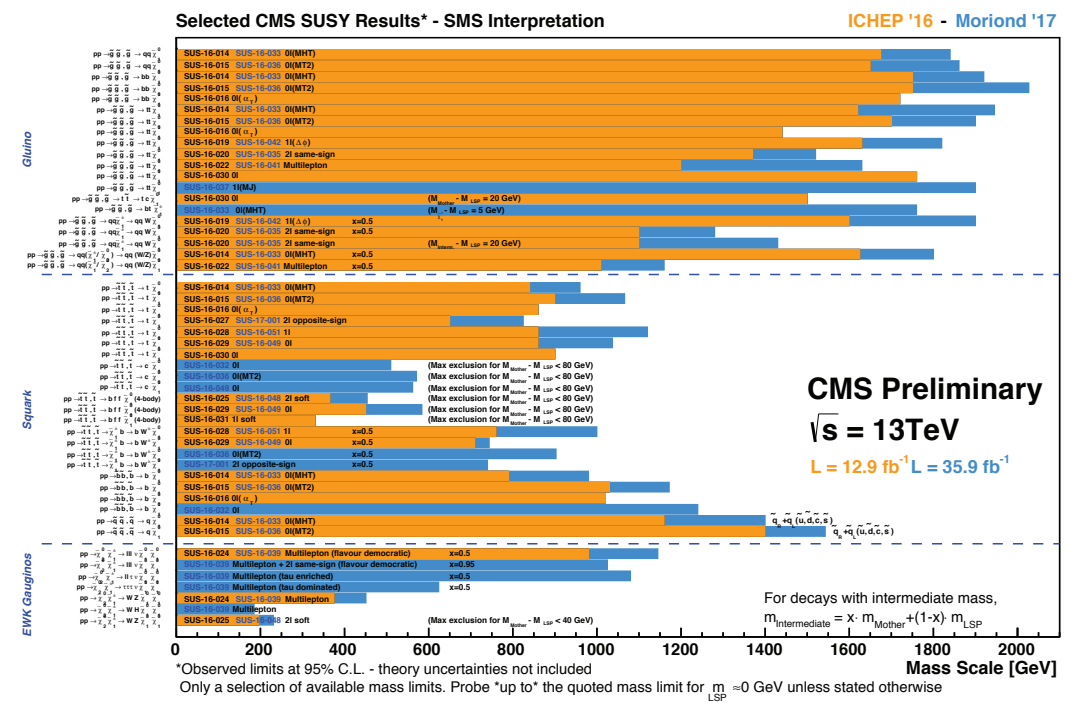

Fig. 3.: Summary of exclusion limits in Simplified Model Spectra (SMS) from CMS searches for SUSY as of March, 2017. Taken from Ref. [210]. 


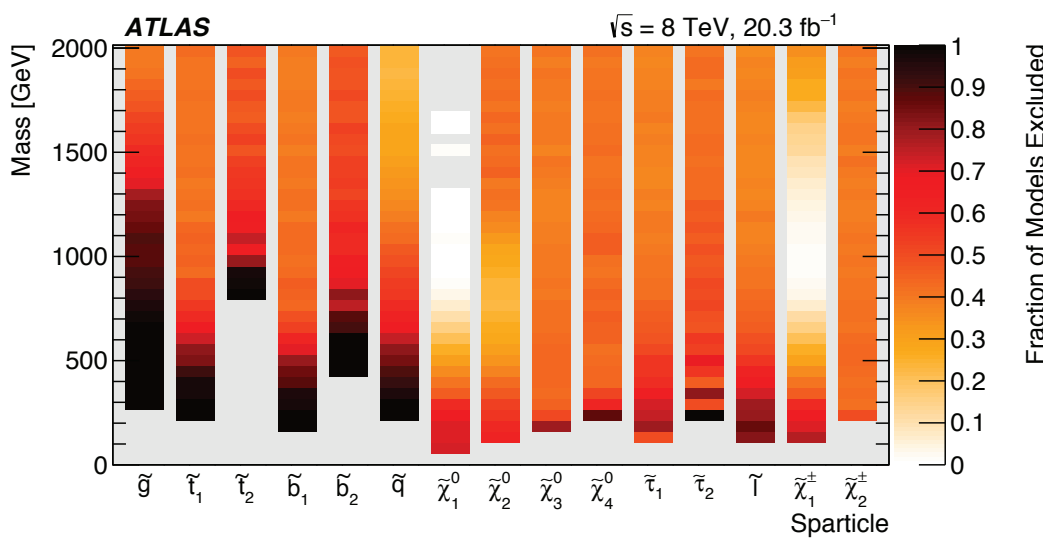

Fig. 4.: A summary of the sensitivity of ATLAS to different types of supersymmetric particles in the 19 parameter pMSSM. Each vertical bar is a 1D projection of the supersymmetric particle mass, with the color coding representing the fraction of models excluded by the ATLAS searches in each bin. This figure taken from Ref. [212].

as of the spring of 2017. Because the LHC is a proton-proton collider, the strongest SUSY mass bounds of about $2 \mathrm{TeV}$ are obtained for the colored superpartners (squarks and gluinos). Bounds on the top squark mass (which play an important role in assessing the degree of fine-tuning required to accommodate the observed Higgs and $Z$ boson masses) are closer to $1 \mathrm{TeV}$. Clearly some tension exists between the theoretical expectations for the magnitude of the SUSY-breaking parameters and the non-observation of supersymmetric phenomena. Hence, the title of this section, which is also the title of Ref. [211], where the theoretical implications of the present LHC data for TeV-scale supersymmetry is reconsidered.

The absence of evidence for supersymmetry in the LHC data can also be interpreted in the context of the pMSSM, which was briefly introduced at the end of Section 8.4. In a scan of the 19 parameter pMSSM performed by the ATLAS Collaboration, the mass of each supersymmetric particle was constrained with an upper limit of $4 \mathrm{TeV}$, motivated to ensure a high density of models in reach of the LHC. Lower limits on the supersymmetric particle masses were also applied to avoid constraints from the LEP experiments. A summary of the sensitivity of the ATLAS Collaboration experiment to different types of supersymmetric particles in the pMSSM is shown in Fig. 4.

Of course, the LHC program is still in its infancy. Two more years of Run-2 data from 2017-2018 must be analyzed. After a two year shutdown, 
Run-3 follows from 2021-2023 according to the current planning schedule. The high luminosity (HL) phase of the LHC [213] will commence in 2026, with an anticipation of reaching $3000 \mathrm{fb}^{-1}$ of data by the year 2038. This is a nearly 100-fold increase of the present LHC data sample. There is still ample room for the discovery of SUSY at the LHC during its lifetime.

Thus, the experimental future of supersymmetry is still very much alive. Beyond the HL-LHC, there are possibilities of energy upgrades at the LHC by roughly a factor of two, and considerations of the next generation of hadron colliders with a center of mass energy of $100 \mathrm{TeV}$. If the SUSYbreaking scale is somewhat higher than $1 \mathrm{TeV}$ (but less than say, $10 \mathrm{TeV}$ ), then opportunities for discovery will be available at these future hadron collider facilities [96].

The theoretical future of supersymmetry is also quite bright. Even if the SUSY-breaking scale lies significantly above the $\mathrm{TeV}$-scale, there are still many opportunities for incorporating supersymmetry into the fundamental theory of particle physics. In this latter scenario, SUSY would not be relevant for explaining the origin of the scale of electroweak symmetry breaking. Another explanation would be required, perhaps one of the other suggested theoretical approaches mentioned in Section 3.

For example, it may still be possible that some remnant of the supersymmetric particle spectrum survives down to the TeV-scale or below. This is the idea of split-SUSY [214-219], in which the squarks and sleptons are significantly heavier (perhaps by many orders of magnitude) than $1 \mathrm{TeV}$, whereas the fermionic superpartners of the gauge and Higgs bosons may be kinematically accessible at the LHC. Of course, the SUSY-breaking dynamics responsible for such a split-SUSY spectrum would not be related to the origin of the scale of electroweak symmetry breaking. Nevertheless, models of split-SUSY can account for the dark matter (which is assumed to be the LSP gaugino or higgsino) and gauge coupling unification, thereby preserving two of the desirable features of $\mathrm{TeV}$-scale supersymmetry.

There are many theoretical aspects of supersymmetry theory that lie beyond the scope of these lectures but deserve further exploration. Among these, non-perturbative approaches to supersymmetric theories, such as holomorphy and Seiberg duality, have been particularly fruitful. The power of holomorphy was briefly exhibited in Section 5.8, when we reviewed Seiberg's proof of the non-renormalization of the superpotential [109]. There are many other applications of holomorphy, such as the computation of exact $\beta$ functions in supersymmetric gauge theories [116, 117, 220]. As an effective tool in non-perturbative regimes, Seiberg duality elucidates 
strongly coupled gauge theories by relating them to dual weakly coupled gauge theories. In Refs. [20, 23, 221-223] one can find numerous applications to the study of non-perturbative dynamics in strongly-coupled supersymmetric theories and in fundamental models of SUSY-breaking.

Another flourishing area of research is that of scattering amplitudes [224, 225], where novel methods are being developed to facilitate computations that were previously intractable using the traditional Feynmandiagrammatic approach. Here supersymmetric theories can serve as testing grounds for techniques that may eventually be extended to nonsupersymmetric quantum field theories. For example, in $N=4$ supersymmetric Yang-Mills theory (one of the few known examples of a finite quantum field theory in four spacetime dimensions), amplitudes are well understood, making it a relatively simple arena in which to study new computational methods [226]. Moreover, tree-level gluon scattering amplitudes in $N=4$ super Yang-Mills are identical to those in any other gauge theory, so it is reasonable to expect that methods developed for SUSY gauge theories could be adapted to the computation of QCD amplitudes.

Supersymmetry is also a powerful tool for analyzing a variety of problems in mathematical physics, and plays a critical role in the formulation of string theory [26, 227-234]. Evidently, even in the absence of evidence for SUSY at the TeV scale, it is very likely that supersymmetry will lead to important new insights, both in experimental and theoretical directions. With this in mind, it is our hope that these lectures have provided a modest introduction into the fascinating world of supersymmetry.

\section{Acknowledgments}

We would like to thank Zackaria Chacko, Andrew Cohen, Michael Dine, Herbi Dreiner, Stephen Martin, Raman Sundrum, and John Terning for many enlightening discussions. H.E.H. is grateful to Rouven Essig and Ian Low for their invitation to present these lectures at TASI 2016, and their patience in waiting for these lecture notes to be completed. This work is supported in part by the U.S. Department of Energy grant number DE-SC0010107. L.S.H. is also supported by the Israel Science Foundation under grant no. 1112/17. 


\section{References}

[1] H.K. Dreiner, H.E. Haber and S.P. Martin, Phys. Rept. 494, 1 (2010) [arXiv:hep-ph/0812.1594].

[2] J. Wess and J. Bagger, Supersymmetry and Supergravity (Princeton University Press, Princeton, NJ, 1992).

[3] S.J. Gates Jr., M.T. Grisaru, M. Roček and W. Siegel, Superspace or One Thousand and One Lessons in Supersymmetry (Benjamin/Cummins Publishing Company, Reading, MA, 1983) [also available at arXiv:hep-th/0108200].

[4] P.P. Srivastava, Supersymmetry, Superfields and Supergravity: an introduction (Adam Hilger, Bristol, UK, 1986).

[5] O. Piguet and K. Sibold, Renormalized Supersymmetry (Birkhäuser, Boston, MA, 1986).

[6] P.G.O. Freund, Introduction to Supersymmetry (Cambridge University Press, Cambridge, UK, 1986).

[7] H.J.W. Müller-Kirsten and A. Wiedemann, Supersymmetry: An Introduction with Conceptual and Calculational Details (World Scientific, Singapore, 1987).

[8] P. West, Introduction to Supersymmetry and Supergravity, extended 2nd edition (World Scientific, Singapore, 1990).

[9] J. Łopuszánski, An Introduction to Symmetry and Supersymmetry in Quantum Field Theory (World Scientific, Singapore, 1991)

[10] D. Bailin and A. Love, Supersymmetric Gauge Field Theory and String Theory (Institute of Physics Publishing, Bristol, UK, 1994).

[11] I.L. Buchbinder and S.M. Kuzenko, Ideas and Methods of Supersymmetry and Supergravity or a Walk through Superspace, revised edition (Institute of Physics Publishing, Bristol, UK, 1995).

[12] S.K. Soni and S. Singh, Supersymmetry: Basics and Concepts (Narosa Publishing House, New Delhi, 2000).

[13] A.S. Galperin, E.A. Ivanov, V.I. Ogievetsky and E.S. Sokatchev, Harmonic Superspace (Cambridge University Press, Cambridge, UK, 2001).

[14] N. Polonsky, Supersymmetry: Structure and phenomena (Springer-Verlag, Berlin, Germany, 2001).

[15] R.N. Mohapatra, Unification and Supersymmetry: The Frontiers of QuarkLepton Physics, 3rd edition (Springer-Verlag, New York, 2003).

[16] M. Drees, R.M. Godbole and P. Roy, Theory and Phenomenology of Sparticles (World Scientific, Singapore, 2004).

[17] H. Baer and X. Tata, Weak Scale Supersymmetry (Cambridge University Press, Cambridge, UK, 2006).

[18] I.J.R. Aitchison, Supersymmetry in Particle Physics: An Elementary Introduction, (Cambridge University Press, Cambridge, UK, 2007).

[19] P. Binétruy, Supersymmetry: Theory, Experiment and Cosmology (Oxford University Press, Oxford, UK, 2006).

[20] J. Terning, Modern Supersymmetry (Oxford University Press, Oxford, UK, 2006).

[21] H.J.W. Müller-Kirsten and A. Wiedemann, Introduction to Supersymmetry, 2nd edition (World Scientific, Singapore, 2010). 
[22] P. Labelle, Supersymmetry Demystified (McGraw Hill,, New York, 2010).

[23] M.A. Shifman, Advanced Topics in Quantum Field Theory (Cambridge University Press, Cambridge, UK, 2012).

[24] D.Z. Freedman and A. Van Proeyen, Supergravity (Cambridge University Press, Cambridge, UK, 2012).

[25] S. Weinberg, The quantum theory of fields: Volume 3: Supersymmetry (Cambridge University Press, Cambridge, UK, 2013).

[26] M. Dine, Supersymmetry and String Theory: Beyond the Standard Model, 2nd edition (Cambridge University Press, Cambridge, UK, 2015)

[27] E.B. Manoukian, Quantum Field Theory II: Introductions to Quantum Gravity, Supersymmetry and String Theory (Springer International Publishing, Cham, Switzerland, 2016).

[28] P. Nath, Supersymmetry, Supergravity, and Unification (Cambridge University Press, Cambridge, UK, 2017).

[29] S. Raby, Supersymmetric Grand Unified Theories (Springer International Publishing, Cham, Switzerland, 2017).

[30] J.G. Taylor, Prog. Part. Nucl. Phys. 12, 1 (1984).

[31] H.P. Nilles, Phys. Rept. 110, 1 (1984).

[32] H.E. Haber and G.L. Kane, Phys. Rept. 117, 75 (1985).

[33] M.F. Sohnius, Phys. Rept. 128, 39 (1985).

[34] A.B. Lahanas and D.V. Nanopoulos, Phys. Rept. 145, 1 (1987).

[35] H.E. Haber, "Introductory low-energy supersymmetry," in Recent Directions in Particle Theory, Proceedings of the Theoretical Advanced Study Institute (TASI-1992), Boulder, CO, edited by J. Harvey and J. Polchinski (World Scientific Publishing Co., Singapore, 1993) pp. 589-686 [arXiv:hep-ph/9306207].

[36] J.-P. Derendinger, "Lecture notes on globally supersymmetric theories in four and two dimensions," in Proceedings of the Hellenic School of Particle Physics, Corfu, Greece, 13-30 September 1989, edited by E.N. Argyres, N. Tracas and G. Zoupanos (World Scientific, Singapore, 1990) pp. 111-243. A link to an electronic version of this manuscript can be found at: http://www.derendinger.itp.unibe.ch/ Documents,_publications,_lecture_notes_files/SUSY_nd.pdf.

[37] J.D. Lykken, "Introduction to Supersymmetry," in Fields, Strings and Duality, Proceedings of the Theoretical Advanced Summer Institute (TASI-1996), Boulder, CO, edited by C. Efthimiou and B. Greene (World Scientific Publishing Co., Singapore, 1997) pp. 85-153.

[38] S.P. Martin, Adv. Ser. Direct. High Energy Phys. 21, 1 (2010). The most recent version of this review can be found in arXiv:hep-ph/9709356.

[39] G.F. Giudice and R. Rattazzi, Phys. Rept. 322, 419 (1999) [arXiv:hep$\mathrm{ph} / 9801271]$.

[40] A. Bilal, "Introduction to supersymmetry," expanded notes of lectures given at the Summer School GIF 2000, hep-th/0101055.

[41] A.Yu. Petrov, "Quantum superfield supersymmetry," hep-th/0106094.

[42] J.M. Figueroa-O'Farrill, "BUSSTEPP lectures on supersymmetry," arXiv: hep-th/0109172.

[43] D.J.H. Chung, L.L. Everett, G.L. Kane, S.F. King, J.D. Lykken and 
L.T. Wang, Phys. Rept. 407, 1 (2005) [arXiv:hep-ph/0312378].

[44] M.A. Luty, "2004 TASI lectures on supersymmetry breaking," in Physics in $D \geq 4$, Proceedings of the Theoretical Advanced Study Institute (TASI2004), Boulder, CO, edited by J. Terning, C.E.M. Wagner, and D. Zeppenfeld (World Scientific Publishing Co., Singapore, 2006) pp. 495-582 [arXiv: hepth/0509029].

[45] M.J. Ramsey-Musolf and S. Su, Phys. Rept. 456, 1 (2008) [arXiv:hep$\mathrm{ph} / 0612057]$.

[46] Y. Shirman, "Introduction to Supersymmetry and Supersymmetry Breaking," in The Dawn of the LHC Era, Proceedings of the Theoretical Advanced Study Institute (TASI-2008), Boulder, CO, edited by T. Han (World Scientific Publishing Co., Singapore, 2010) pp. 359-422 [arXiv:hep-ph/0907.0039].

[47] G.L. Kane, editor, Perspectives in Supersymmetry II (World Scientific Publishing Co., Singapore, 2010).

[48] H.E. Haber, Supersymmetry, Part I (Theory), in the 2016 Review of Particle Physics, in the 2016 Review of Particle Physics, C. Patrignani et al. [Particle Data Group], Chin. Phys. C 40, 100001 (2016) and 2017 update. The update can be found at http://pdg.lbl.gov/.

[49] D. Bertolini, J. Thaler and Z. Thomas, "Super-Tricks for Superspace," in Searching for New Physics at Small and Large Scales, Proceedings of the Theoretical Advanced Study Institute (TASI-2012), Boulder, CO, edited by M. Schmaltz and E. Pierpaoli (World Scientific Publishing Co., Singapore, 2013) pp. 421-496 [arXiv:hep-ph/1302.6229].

[50] R.U. Sexl and H.K. Urbantke, Relativity, Groups, Particles: Special Relativity and Relativistic Symmetry in Field and Particle Physics (Springer-Verlag, Vienna, Austria, 2001).

[51] T. Takagi, Japan J. Math. 1, 83 (1925).

[52] R.A. Horn and C.R. Johnson, Matrix Analysis (Cambridge University Press, Cambridge, UK, 1990).

[53] R.A. Horn and C.R. Johnson, Topics in Matrix Analysis (Cambridge University Press, Cambridge, UK, 1991).

[54] R.A. Horn and C.R. Johnson, Matrix Analysis, 2nd edition (Cambridge University Press, Cambridge, UK, 2013).

[55] L. Autonne, Annales de l'Université de Lyon, Nouvelle Série I, Fasc. 38, 1 (1915).

[56] P. Minkowski, Phys. Lett. 67B, 421 (1977).

[57] M. Gell-Mann, P. Ramond and R. Slansky, in Supergravity, edited by D. Freedman and P. van Nieuwenhuizen (North-Holland Publishing Company, Amsterdam, 1979) p. 315.

[58] T. Yanagida, in Proceedings of the Workshop on Unified Theory and Baryon Number in the Universe, edited by O. Sawada and A. Sugamoto (KEK, Tsukuba, Japan, 1979) p. 95.

[59] R.N. Mohapatra and G. Senjanovic, Phys. Rev. Lett. 44, 912 (1980); Phys. Rev. D23, 165 (1981).

[60] J. Schechter and J.W.F. Valle, Phys. Rev. D22 , 2227 (1980); D25, 774 (1982). 
[61] E.I. Gates and K.L. Kowalski, Phys. Rev. D37, 938 (1988).

[62] A. Denner, H. Eck, O. Hahn and J. Kublbeck, Phys. Lett. B291, 278 (1992); Nucl. Phys. B387, 467 (1992).

[63] R. Kleiss, I. Malamos and G. van den Oord, Eur. Phys. J. C64, 387 (2009) [arXiv:hep-ph/0906.3388].

[64] P. Langacker, The Standard Model and Beyond, 2nd edition (CRC Press, Boca Raton, FL, 2017).

[65] See e.g., K. Nakamura and S.T. Petkov, Neutrino mass, mixing, and oscillations, in the 2016 Review of Particle Physics, C. Patrignani et al. [Particle Data Group], Chin. Phys. C 40, 100001 (2016) and 2017 update. The update can be found at http://pdg.lbl.gov/.

[66] See e.g., M. Drees and G. Gerbier, Dark Matter, in the 2016 Review of Particle Physics, op. cit.

[67] See e.g., J.E. Kim and G. Carosi, Rev. Mod. Phys. 82, 557 (2010).

[68] See e.g., A. Hebecker and J. Hisano, Grand Unified Theories, in the 2016 Review of Particle Physics, op. cit.

[69] See e.g., G.A. White, A Pedagogical Introduction to Electroweak Baryogenesis (IOP Publishing, Bristol, UK, 2016).

[70] See e.g., J. Ellis and D. Wands, Inflation, in the 2016 Review of Particle Physics, op. cit.

[71] See e.g., M.J. Mortonso, D.H. Weinberg and M. White, Dark Energy, in the 2016 Review of Particle Physics, op. cit.

[72] See, e.g., A.A. Petrov and A.E. Blechman, Effective Field Theories (World Scientific Publishing Co. Singapore, 2016).

[73] V.F. Weisskopf, Phys. Rev. 56, 72 (1939).

[74] V.F. Weisskopf, Zeit. für Physik 89, 27 (1934) [Erratum: 90, 817 (1934)].

[75] E. Witten, Nucl. Phys. B 188, 513 (1981).

[76] L. Susskind, Phys. Rept. 104, 181 (1984).

[77] See e.g., C. Csaki, C. Grojean and J. Terning, Rev. Mod. Phys. 88 (2016) 045001.

[78] L. Randall and R. Sundrum, Phys. Rev. Lett. 83, 3370 (1999).

[79] See e.g., J. Parsons and A. Pomarol, Extra Dimensions, in the 2016 Review of Particle Physics, op. cit.

[80] W.A. Bardeen, "On naturalness in the standard model," FERMILABCONF-95-391-T

[81] K.A. Meissner and H. Nicolai, Phys. Lett. B 648, 312 (2007) [arXiv:hepth/0612165].

[82] S. Iso, N. Okada and Y. Orikasa, Phys. Lett. B 676, 81 (2009) [arXiv:hep/ph/0902.4050].

[83] G. Marques Tavares, M. Schmaltz and W. Skiba, Phys. Rev. D 89, 015009 (2014) [arXiv:hep-ph/1308.0025].

[84] A. Gorsky, A. Mironov, A. Morozov and T.N. Tomaras, J. Exp. Theor. Phys. 120, 344 (2015) [Zh. Eksp. Teor. Fiz. 147, 399 (2015)] [arXiv:hep$\mathrm{ph} / 1409.0492]$.

[85] A.J. Helmboldt, P. Humbert, M. Lindner and J. Smirnov, JHEP 1707, 113 (2017) [arXiv:hep-ph/1603.03603]. 
[86] S.R. Coleman and E.J. Weinberg, Phys. Rev. D 7, 1888 (1973).

[87] V. Agrawal, S.M. Barr, J.F. Donoghue and D. Seckel, Phys. Rev. Lett. 80 (1998) 1822; Phys. Rev. D 57 (1998) 5480.

[88] P.W. Graham, D.E. Kaplan and S. Rajendran, Phys. Rev. Lett. 115, 221801 (2015) [arXiv:hep-ph/1504.07551]

[89] N. Arkani-Hamed, T. Cohen, R.T. D'Agnolo, A. Hook, H.D. Kim and D. Pinner, Phys. Rev. Lett. 117, 251801 (2016) [arXiv:hep-ph/1607.06821].

[90] H. Murayama, Supersymmetry, in the Proceedings of 22nd INS International Symposium on Physics with High Energy Colliders, Tokyo, Japan, 8-10 Mar 1994, edited by S. Yamada and T. Ishii (World Scientific Publishing Co., Singapore, 1995) pp. 357-379 [arXiv:hep-ph/9410285].

[91] H. Murayama, Int. J. Mod. Phys. A 19, 1265 (2004).

[92] See e.g., O. Buchmüller and P. de Jong, Supersymmetry, Part II (Experiment), in the 2016 Review of Particle Physics, op. cit.

[93] R. Barbieri and A. Strumia, arXiv:hep-ph/0007265.

[94] L. Giusti, A. Romanino and A. Strumia, Nucl. Phys. B 550, 3 (1999).

[95] H.C. Cheng and I. Low, JHEP 0309, 051 (2003); 0408, 061 (2004).

[96] M.L. Mangano, editor, Physics at the FCC-hh, a $100 \mathrm{TeV}$ pp collider, CERN Yellow Report, CERN-2017-003-M (2017).

[97] J. Wess and B. Zumino, Nucl. Phys. B 70, 39 (1974).

[98] S.R. Coleman and J. Mandula, Phys. Rev. 159, 1251 (1967).

[99] R. Haag, J.T. Łopuszański and M. Sohnius, Nucl. Phys. B 88, 257 (1975).

[100] A. Salam and J.A. Strathdee, Nucl. Phys. B 80, 499 (1974).

[101] E. Sokatchev, Nucl. Phys. B 99, 96 (1975).

[102] P. Roman, Introduction to quantum field theory, (John Wiley \& Sons, Inc., New York, NY, 1969).

[103] B. de Wit, Phys. Rev. D 11, 898 (1975).

[104] E. Witten, Nucl. Phys. B 202, 253 (1982).

[105] S. Ferrara, J. Wess and B. Zumino, Phys. Lett. 51B, 239 (1974).

[106] A. Salam and J.A. Strathdee, Phys. Rev. D 11, 1521 (1975).

[107] A. Salam and J.A. Strathdee, Fortsch. Phys. 26, 57 (1978).

[108] M.T. Grisaru, W. Siegel and M. Roček, Nucl. Phys. B 159, 429 (1979).

[109] N. Seiberg, Phys. Lett. B 318, 469 (1993) [arXiv:hep-ph/9309335].

[110] See e.g., B. Hall, Lie Groups, Lie Algebras, and Representations, 2nd edition (Springer International Publishing, Cham, Switzerland, 2015).

[111] F.A. Berezin, The Method of Second Quantization (Academic Press, Inc., New York, NY, 1966).

[112] S. Pokorski, Gauge Field Theories, 2nd edition (Cambridge University Press, Cambridge, UK, 2000).

[113] P.C. West, Phys. Lett. B 258, 375 (1991).

[114] I. Jack, D.R.T. Jones and P.C. West, Phys. Lett. B 258, 382 (1991).

[115] D.C. Dunbar, I. Jack and D.R.T. Jones, Phys. Lett. B 261, 62 (1991).

[116] M.A. Shifman and A.I. Vainshtein, Nucl. Phys. B 277, 456 (1986).

[117] M.A. Shifman and A.I. Vainshtein, Nucl. Phys. B 359, 571 (1991).

[118] E. Poppitz and L. Randall, Phys. Lett. B 389, 280 (1996) [arXiv:hepth/9608157]. 
[119] J.W. Cui, Y. Tang and Y.L. Wu, Phys. Rev. D 79, 125008 (2009) [arXiv:hep-ph/0812.0892].

[120] J. Wess and B. Zumino, Nucl. Phys. B 78, 1 (1974).

[121] P. Fayet and J. Iliopoulos, Phys. Lett. 51B, 461 (1974).

[122] B.A. Ovrut and J. Wess, Phys. Rev. D 25, 409 (1982).

[123] R.D.C. Miller, Phys. Lett. B 129, 72 (1983).

[124] M. Dine, P. Draper, H.E. Haber and L. Stephenson Haskins, Phys. Rev. D 94, 095003 (2016) [arXiv:hep-th/1607.06995].

[125] L. O'Raifeartaigh, Nucl. Phys. B 96, 331 (1975).

[126] A. Salam and J.A. Strathdee, Phys. Lett. 49B, 465 (1974).

[127] S. Ferrara, L. Girardello and F. Palumbo, Phys. Rev. D 20, 403 (1979).

[128] S. Deser and B. Zumino, Phys. Rev. Lett. 38, 1433 (1977).

[129] E. Cremmer, B. Julia, J. Scherk, P. van Nieuwenhuizen, S. Ferrara and L. Girardello, Phys. Lett. 79B, 231 (1978).

[130] E. Cremmer, S. Ferrara, L. Girardello and A. Van Proeyen, Nucl. Phys. B 212, 413 (1983).

[131] E. Cremmer, S. Ferrara, L. Girardello and A. Van Proeyen, Phys. Lett. 116B, 231 (1982).

[132] L.J. Hall, J.D. Lykken and S. Weinberg, Phys. Rev. D 27, 2359 (1983).

[133] L. Randall and R. Sundrum, Nucl. Phys. B 557, 79 (1999) [arXiv:hepth/9810155].

[134] F. D'Eramo, J. Thaler and Z. Thomas, JHEP 1206, 151 (2012) [arXiv:hepph/1202.1280]; 1309, 125 (2013) [arXiv:hep-ph/1307.3251].

[135] K. Harigaya and M. Ibe, Phys. Rev. D 90, 085028 (2014) [arXiv:hepth/1409.5029].

[136] L. Girardello and M.T. Grisaru, Nucl. Phys. B194, 65 (1982).

[137] S.P. Martin, Phys. Rev. D 61, 035004 (2000) [arXiv:hep-ph/9907550].

[138] L.J. Hall and L. Randall, Phys. Rev. Lett. 65, 2939 (1990).

[139] I. Jack and D.R.T. Jones, Phys. Lett. B 457, 101 (1999) [arXiv:hep$\mathrm{ph} / 9903365]$.

[140] C.S. Ün, S.H. Tanyıldızı, S. Kerman and L. Solmaz, Phys. Rev. D 91, 105033 (2015) [arXiv:hep-ph/1412.1440].

[141] U. Chattopadhyay and A. Dey, JHEP 1610, 027 (2016) [arXiv:hepph/1604.06367].

[142] G.G. Ross, K. Schmidt-Hoberg and F. Staub, Phys. Lett. B 759, 110 (2016) [arXiv:hep-ph/1603.09347]; JHEP 1703, 021 (2017) [arXiv:hepph/1701.03480].

[143] See, e.g., H.E. Haber, "Lectures on Electroweak Symmetry Breaking," in Testing the Standard Model, Proceedings of the Theoretical Advanced Study Institute (TASI-1990), Boulder, CO, edited by M. Cvetič and P. Langacker (World Scientific Publishing Co., Singapore, 1991) pp. 340-475.

[144] A. Pomarol and S. Dimopoulos, Nucl. Phys. B 453, 83 (1995) [arXiv:hep$\mathrm{ph} / 9505302]$.

[145] R. Rattazzi, Phys. Lett. B 375, 181 (1996) [arXiv:hep-ph/9507315].

[146] A. Dedes, H.E. Haber and J. Rosiek, JHEP 0711, 059 (2007) [arXiv:hepph/0707.3718]. 
[147] P. Langacker, Phys. Rept. 72, 185 (1981).

[148] R.A. Bertlmann, Anomalies in Quantum Field Theory (Oxford University Press, Oxford, UK, 1996)

[149] S. Weinberg, Phys. Rev. Lett. 43, 1566 (1979); Phys. Rev. D 22, 1694 (1980).

[150] F. Wilczek and A. Zee, Phys. Rev. Lett. 43, 1571 (1979).

[151] H.A. Weldon and A. Zee, Nucl. Phys. B 173, 269 (1980).

[152] P. Fayet, Phys. Lett. 64B, 159 (1976).

[153] G.R. Farrar and P. Fayet, Phys. Lett. 76B, 575 (1978).

[154] L. Alvarez-Gaume, J. Polchinski and M.B. Wise, Nucl. Phys. B 221, 495 (1983).

[155] J.M. Frere, D.R.T. Jones and S. Raby, Nucl. Phys. B 222, 11 (1983).

[156] J.P. Derendinger and C.A. Savoy, Nucl. Phys. B 237, 307 (1984).

[157] J.F. Gunion, H.E. Haber and M. Sher, Nucl. Phys. B 306, 1 (1988).

[158] D. Chowdhury, R.M. Godbole, K.A. Mohan and S.K. Vempati, JHEP 1402, 110 (2014) [arXiv:hep-ph/1310.1932].

[159] W.G. Hollik, JHEP 1608, 126 (2016) [arXiv:hep-ph/1606.08356].

[160] J.A. Casas, A. Lleyda and C. Munoz, Nucl. Phys. B 471, 3 (1996) [arXiv:hep-ph/9507294].

[161] S. Dimopoulos and D.W. Sutter, Nucl. Phys. B 452, 496 (1995) [arXiv:hepph/9504415]; D.W. Sutter, Stanford Ph. D. thesis [arXiv:hep-ph/9704390].

[162] H.E. Haber, Nucl. Phys. Proc. Suppl. 101, 217 (2001) [arXiv:hep$\mathrm{ph} / 0103095]$.

[163] H. Georgi, Phys. Lett. 169B, 231 (1986).

[164] L.J. Hall, V.A. Kostelecky and S. Raby, Nucl. Phys. B 267, 415 (1986).

[165] For a review, see e.g., S. Khalil, Int. J. Mod. Phys. A 18, 1697 (2003) [arXiv:hep-ph/0212050].

[166] A. Djouadi, J.L. Kneur and G. Moultaka, Comput. Phys. Commun. 176, 426 (2007) [arXiv:hep-ph/0211331];

[167] C.F. Berger, J.S. Gainer, J.L. Hewett and T.G. Rizzo, JHEP 0902, 023 (2009) [arXiv:hep-ph/0812.0980].

[168] K.J. de Vries et al., Eur. Phys. J. C 75, 422 (2015) [arXiv:hep$\mathrm{ph} / 1504.03260]$.

[169] J. Berger, M.W. Cahill-Rowley, D. Ghosh, J.L. Hewett, A. Ismail and T.G. Rizzo, Phys. Rev. D 93, 035017 (2016) [arXiv:hep-ph/1510.08840].

[170] J.F. Gunion and H.E. Haber, Nucl. Phys. B 272, 1 (1986) Erratum: [Nucl. Phys. B 402, 567 (1993)]; Nucl. Phys. B 278, 449 (1986); 307, 445 (1988) Erratum: [Nucl. Phys. B 402, 569 (1993)].

[171] J.F. Gunion, H.E. Haber, G. Kane and S. Dawson, The Higgs Hunter's Guide (Westview Press, Boulder, CO, 2000).

[172] A. Djouadi, Phys. Rept. 459, 1 (2008) [arXiv:hep-ph/0503173].

[173] J. Bernon, J.F. Gunion, H.E. Haber, Y. Jiang and S. Kraml, Phys. Rev. D 92, 075004 (2015) [arXiv:hep-ph/1507.00933].

[174] L.J. Hall and M.B. Wise, Nucl. Phys. B 187, 397 (1981).

[175] H.E. Haber and Y. Nir, Nucl. Phys. B 335, 363 (1990).

[176] J.F. Gunion and H.E. Haber, Phys. Rev. D 67, 075019 (2003) [arXiv:hep- 
$\mathrm{ph} / 0207010]$.

[177] H.E. Haber and R. Hempfling, Phys. Rev. Lett. 66, 1815 (1991); Phys. Rev. D 48, 4280 (1993) [hep-ph/9307201].

[178] Y. Okada, M. Yamaguchi and T. Yanagida, Prog. Theor. Phys. 85, 1 (1991).

[179] J.R. Ellis, G. Ridolfi and F. Zwirner, Phys. Lett. B 257, 83 (1991).

[180] H.E. Haber, R. Hempfling and A.H. Hoang, Zeit. für Physik C 75, 539 (1997) [arXiv:hep-ph/9609331].

[181] For a review and references to the original literature, see P. Draper and H. Rzehak, Phys. Rept. 619, 1 (2016) [arXiv:hep-ph/1601.01890].

[182] M. Carena and H.E. Haber, Prog. Part. Nucl. Phys. 50, 63 (2003) [arXiv:hep-ph/0208209].

[183] G.G. Ross, Grand Unified Theories (Westview Press, Boulder, CO, 1985).

[184] D.J. Castano, E.J. Piard and P. Ramond, Phys. Rev. D 49, 4882 (1994) [arXiv:hep-ph/9308335].

[185] W. Martens, "Threshold Corrections in Grand Unified Theories," KIT Dissertation, https://publikationen.bibliothek.kit.edu/1000023673.

[186] B.C. Allanach, A. Bednyakov and R. Ruiz de Austri, Comput. Phys. Commun. 189, 192 (2015) [arXiv:hep-ph/1407.6130].

[187] V. Lucas and S. Raby, Phys. Rev. D 54, 2261 (1996) [arXiv:hep$\mathrm{ph} / 9601303]$.

[188] J. Rosiek, Phys. Rev. D 41, 3464 (1990) [Erratum: hep-ph/9511250]. The most recent corrected version of this manuscript can be found here: http://www.fuw.edu.pl/〜rosiek/physics/prd41.html.

[189] C. Patrignani et al. [Particle Data Group], 2016 Review of Particle Physics, Chin. Phys. C 40, 100001 (2016) and 2017 update. The update can be found at http://pdg.lbl.gov/.

[190] Y. Grossman and S. Rakshit, Phys. Rev. D 69, 093002 (2004) [arXiv:hep$\mathrm{ph} / 0311310]$.

[191] A. Dedes, S. Rimmer and J. Rosiek, JHEP 0608, 005 (2006) [arXiv:hep$\mathrm{ph} / 0603225]$.

[192] E. Peinado and A. Vicente, Phys. Rev. D 86, 093024 (2012) [arXiv:hep$\mathrm{ph} / 1207.6641]$

[193] J. Hisano, T. Moroi, K. Tobe, M. Yamaguchi and T. Yanagida, Phys. Lett. B 357, 579 (1995) [arXiv:hep-ph/9501407].

[194] J. Hisano, T. Moroi, K. Tobe and M. Yamaguchi, Phys. Rev. D 53, 2442 (1996) [arXiv:hep-ph/9510309].

[195] Y. Grossman and H.E. Haber, Phys. Rev. Lett. 78, 3438 (1997) [arXiv:hep$\mathrm{ph} / 9702421]$.

[196] J.A. Casas and A. Ibarra, Nucl. Phys. B 618, 171 (2001) [arXiv:hep$\mathrm{ph} / 0103065]$.

[197] J.R. Ellis, J. Hisano, M. Raidal and Y. Shimizu, Phys. Rev. D 66, 115013 (2002) [arXiv:hep-ph/0206110].

[198] A. Masiero, S.K. Vempati and O. Vives, New J. Phys. 6, 202 (2004) [arXiv:hep-ph/0407325].

[199] E. Arganda, A.M. Curiel, M.J. Herrero and D. Temes, Phys. Rev. D 71 035011 (2005) [arXiv:hep-ph/0407302]. 
[200] F.R. Joaquim and A. Rossi, Phys. Rev. Lett. 97, 181801 (2006) [arXiv:hepph/0604083].

[201] J.R. Ellis and O. Lebedev, Phys. Lett. B 653, 411 (2007) [arXiv:hepph/0707.3419].

[202] J.E. Kim and H.P. Nilles, Phys. Lett. 138B, 150 (1984).

[203] J.E. Kim and H.P. Nilles, Mod. Phys. Lett. A 9, 3575 (1994) [arXiv:hep$\mathrm{ph} / 9406296]$.

[204] G.F. Giudice and A. Masiero, Phys. Lett. B 206, 480 (1988).

[205] J.A. Casas and C. Munoz, Phys. Lett. B 306, 288 (1993) [arXiv:hep$\mathrm{ph} / 9302227$.

[206] G.R. Dvali, G.F. Giudice and A. Pomarol, Nucl. Phys. B 478, 31 (1996) [arXiv:hep-ph/9603238].

[207] M. Maniatis, Int. J. Mod. Phys. A 25, 3505 (2010) [arXiv:hep$\mathrm{ph} / 0906.0777]$.

[208] U. Ellwanger, C. Hugonie and A.M. Teixeira, Phys. Rept. 496, 1 (2010) [arXiv:hep-ph/0910.1785].

[209] For results of the ATLAS supersymmetry searches, see: https://twiki. cern.ch/twiki/bin/view/AtlasPublic/SupersymmetryPublicResults.

[210] Results of the CMS supersymmetry searches can be found here: https://twiki.cern.ch/twiki/bin/view/CMSPublic/PhysicsResultsSUS.

[211] G.G. Ross, Eur. Phys. J. C 74, 2699 (2014).

[212] W.J. Fawcett [for the ATLAS and CMS Collaborations], "pMSSM studies with ATLAS and CMS," in the Proceedings, 4th Large Hadron Collider Physics Conference (LHCP 2016), Lund, Sweden, June 13-18, 2016 edited by J. Bijnens, A. Hoecker and J. Olsen, PoS (LHCP2016) 146.

[213] G. Apollinari, O. Brüning, T. Nakamoto and L. Rossi, CERN Yellow Report, Volume 5, CERN-2015-005 (CERN, Geneva, 2015) [arXiv:1705.08830 [physics.acc-ph]].

[214] N. Arkani-Hamed and S. Dimopoulos, JHEP 0506, 073 (2005) [arXiv:hepth/0405159].

[215] G.F. Giudice and A. Romanino, Nucl. Phys. B 699, 65 (2004) [Erratum: Nucl. Phys. B 706, 487 (2005)] [arXiv:hep-ph/0406088].

[216] N. Arkani-Hamed, S. Dimopoulos, G.F. Giudice and A. Romanino, Nucl. Phys. B 709, 3 (2005) [arXiv:hep-ph/0409232].

[217] J.D. Wells, Phys. Rev. D 71, 015013 (2005) [arXiv:hep-ph/0411041].

[218] A. Arvanitaki, N. Craig, S. Dimopoulos and G. Villadoro, JHEP 1302, 126 (2013) [arXiv:hep-ph/1210.0555].

[219] N. Arkani-Hamed, A. Gupta, D.E. Kaplan, N. Weiner and T. Zorawski [arXiv:hep-ph/1212.6971].

[220] N. Arkani-Hamed and H. Murayama, JHEP 0006, 030 (2000) [arXiv:hepth/9707133].

[221] K.A. Intriligator and N. Seiberg, "Lectures on Supersymmetry Breaking," Class. Quant. Grav. 24, S741 (2007) [Les Houches 87, 125 (2008)] [arXiv:hep$\mathrm{ph} / 0702069]$.

[222] J. Terning, "Nonperturbative supersymmetry," in Particle Physics and Cosmology: The Quest for Physics Beyond the Standard Model(s), Proceed- 
ings of the Theoretical Advanced Study Institute (TASI-2002), Boulder, CO, edited by H.E. Haber and A. Nelson (World Scientific Publishing Co., Singapore, 2004) pp. 343-443 [arXiv:hep-th/0306119].

[223] M.A. Shifman, Prog. Part. Nucl. Phys. 39, 1 (1997) [arXiv:hepth/9704114].

[224] J.M. Henn and J.C. Plefka, Scattering Amplitudes in Gauge Theories (Springer-Verlag, Berlin, Germany, 2014).

[225] H. Elvang and Y.T. Huang, Scattering Amplitudes in Gauge Theory and Gravity (Cambridge University Press, Cambridge, UK, 2015).

[226] M. Spradlin, "Amplitudes in $N=4$ Super-Yang-Mills Theory," in Journeys Through the Precision Frontier-Amplitudes for Colliders Proceedings of the Theoretical Advanced Study Institute (TASI-2014), Boulder, CO, edited by L. Dixon and F. Petriello (World Scientific Publishing Co., Singapore, 2015) pp. 341-361.

[227] M.B. Green, J.H. Schwarz and E. Witten, Superstring Theory, Volume 1: Introduction, and Volume II: Loop Amplitudes, Anomalies and Phenomenology (Cambridge University Press, Cambridge, UK, 1987).

[228] J. Polchinski, String theory, Volume I: Introduction to the Bosonic String, and Volume II: Superstring theory and beyond (Cambridge University Press, Cambridge, UK, 2001).

[229] K. Becker and M. Becker and J.H. Schwarz String Theory and M-Theory: A Modern Introduction (Cambridge University Press, Cambridge, UK, 2007).

[230] E. Kiritsis, String Theory in a Nutshell (Princeton University Press, Princeton, NJ, 2007).

[231] B. Zwiebach, A First Course in String Theory, 2nd Edition (Cambridge University Press, Cambridge, UK, 2009).

[232] L.E. Ibáñez and A.M. Uranga, String Theory and Particle Physics (Cambridge University Press, Cambridge, UK, 2012).

[233] R. Blumenhagen, D. Lüst and S. Theisen, Basic Concepts of String Theory (Springer-Verlag, Berlin, Germany, 2013).

[234] V. Schomerus, A Primer on String Theory (Cambridge University Press, Cambridge, UK, 2017). 